\title{
Computational investigation of short pulse laser-induced modification of surface microstructure and photoacoustic control of surface diffusion
}

\author{
Chengping Wu \\ Anqing, Anhui, P.R.China
}

B. S., University of Science and Technology of China, 2007

\author{
A Dissertation presented to the Graduate Faculty \\ of the University of Virginia in Candidacy for the Degree of \\ Doctor of Philosophy \\ Department of Physics \\ University of Virginia \\ June, 2013
}

Approved by the Examining Committee:

Prof. Leonid. V. Zhigilei, Thesis Advisor

Prof. Joseph Poon, Member

Prof. Eugene Kolomeisky, Member

Prof. Petra Reinke, Member

Prof. James M. Howe, Member

Prof. Giovanni Zangari, Member 


\section{CONTENT}

LIST OF TABLES Vi - vi

LIST OF FIGURES Vii

ACKNOWLEDGEMENTS Xxiii

LIST OF PUBLICATIONS Xxiv

LIST OF PRESENTATIONS XXvi

$\begin{array}{ll}\text { ABSTRACT } & \text { xxviii }\end{array}$

$\begin{array}{ll}\text { 1. Introduction } & 1\end{array}$

1.1 Part I: Short pulse laser interaction with metals 2

1.2 Part II: short pulse laser-induced photoacoustic control of surface diffusion 2

PART I: Short pulse laser interaction with metals $\quad 4$

2. Introduction $\quad 5$

2.1 Background 5

2.2 Introduction to short pulse laser-metal interaction process: two temperature model 6

2.3 The combined TTM-MD model 10

2.4 The TTM parameters $C_{e}, G, K_{e}, S(z, t)$ and $C_{l} \quad 13$

2.5 The interatomic potential for the metals 14

2.6 Contributions 17

3. Runaway lattice-mismatched interface in an atomistic simulation of femtosecond $\begin{array}{ll}\text { laser irradiation of } \mathbf{A g} \text { film - Cu substrate system } & 19\end{array}$

$\begin{array}{ll}3.1 \text { Introduction } & 19\end{array}$

3.2 Computational model 19

$\begin{array}{ll}3.3 \text { Results and discussions } & 24\end{array}$

3.3.1 Laser heating and sub-surface melting $\quad 24$ 
$\begin{array}{ll}\text { 3.3.2 Cooling and resolidification } & 28\end{array}$

3.3.3 Structure and resolidified region 32

3.3.4 Structure of runaway lattice-mismatched interface 39

3.4 Summary 41

4. BCC Cu formation and its stability 44

4.1 Introduction 44

$4.2200 \mathrm{fs}$ laser irradiation of $20 \mathrm{~nm} \mathrm{Cu}$ film - Ag (001) substrate system 46

4.2.1 Threshold fluence for melting of the whole $\mathrm{Cu}$ film 46

4.2.2 Epitaxial growth of $\mathrm{BCC} \mathrm{Cu}$ on $\mathrm{Ag}(001)$ face during resolidification $\quad 48$

4.2.3 Stability of the generated BCC Cu film 51

$4.3200 \mathrm{fs}$ laser irradiation of $5 \mathrm{~nm} \mathrm{Ag}$ film-5 nm Cu film-Ag (001) substrate system 53

4.3.1 Threshold fluence for the complete melting of the Ag film - $\mathrm{Cu}$ film $\quad 54$

4.3.2 Melting of $\mathrm{Ag}$ and $\mathrm{Cu}$ film 56

4.3.3 Resolidfication: epitaxial growth of $\mathrm{Cu}$ on $\mathrm{Ag}(001)$ and subsequent $\begin{array}{ll}\text { growth of } \mathrm{Ag} \text { on } \mathrm{Cu} & 57\end{array}$

4.3.4 Stability of the intermediate BCC Cu film 58

$\begin{array}{ll}\text { 4.4 Concluding remarks } & 60\end{array}$

5. Femtosecond laser ablation of $\mathrm{Ag}(001)$ - experiments and small-scale simulations 62

$\begin{array}{ll}5.1 \text { Introduction } & 62\end{array}$

5.2 Single-Shot femtosecond laser ablation experiments 63

5.3 TTM-MD simulations of single-shot laser (100 fs) irradiation of Ag (001) target 67

$\begin{array}{lll}\text { 5.3.1 Threshold fluence for laser melting } & 68\end{array}$

$\begin{array}{ll}\text { 5.3.2 Threshold fluence for spallation or void formation } & 70\end{array}$

$\begin{array}{lll}\text { 5.3.3 Photomechanical effects } & 72\end{array}$

5.3.4 Size dependence of simulation results $\quad 73$ 
$\begin{array}{lll}\text { 5.3.5 Transition from spallation to phase explosion } & 74\end{array}$

$\begin{array}{ll}5.4 \text { Discussions } & 76\end{array}$

6. Surface swelling and spallation in large-scale simulations of 100 fs laser

$\begin{array}{ll}\text { irradiation of } \mathrm{Ag}(\mathbf{0 0 1}) & 79\end{array}$

$\begin{array}{ll}\text { 6.1 Introduction } & 79\end{array}$

6.2 Threshold fluence for void formation $\quad 80$

6.3 Laser irradiation at $0.085 \mathrm{~J} / \mathrm{cm}^{2}$ : void formation, but no spallation 83

6.3.1 Void nucleation, growth, coalescence and contraction 85

6.3.2 Voids captured (frozen) by heterogeneous resolidification 88

6.3.3 Homogeneous crystal nucleation to form nano-crystalline structure $\quad 90$

6.3.4 Microstructure of the top surface layer 91

6.4 Laser irradiation at $0.09 \mathrm{~J} / \mathrm{cm}^{2}$ : spallation and generation of a frozen nanospike $\quad 97$

$\begin{array}{lll}\text { 6.4.1 Photomechanical spallation } & 97\end{array}$

$\begin{array}{lll}\text { 6.4.2 Structure of the nanospike } & 101\end{array}$

$\begin{array}{ll}6.5 \text { Discussions } & 105\end{array}$

$\begin{array}{lll}\text { 6.5.1 Surface swelling } & 105\end{array}$

$\begin{array}{lll}\text { 6.5.2 Microstructure } & 107\end{array}$

$\begin{array}{lll}\text { 6.5.3 Complex surface morphology } & 107\end{array}$

PART II: Short pulse laser-induced photoacoustic control of surface diffusion 109

$\begin{array}{lr}\text { 7. Introduction } & 110\end{array}$

8. Acoustic enhancement of surface diffusion 114

$\begin{array}{ll}8.1 \text { Introduction } & 114\end{array}$

8.2 Mechanisms of acoustic activation of surface diffusion $\quad 115$

8.2.1 Modification of diffusion barriers by surface strain $\quad 115$

8.2.2 Temperature variation due to the adiabatic heating and cooling 118 
8.2.3 Diffusion enhancement via diffusion barrier and adiabatic $\begin{array}{ll}\text { temperature variation } & 121\end{array}$

8.2.4 Dynamic coupling between adsorbates and SAW $\quad 125$

$\begin{array}{ll}\text { 8.3 Concluding remarks } & 128\end{array}$

9. Mechanism of acoustically induced diffusional structuring of surface adatoms 130

$\begin{array}{ll}9.1 \text { Introduction } & 130\end{array}$

$\begin{array}{ll}\text { 9.2 The role of damping } & 131\end{array}$

$\begin{array}{ll}\text { 9.3 Diffusional mechanism } & 133\end{array}$

$\begin{array}{ll}9.4 \text { Conclusions } & 141\end{array}$

10. Summary of the dissertation $\quad 142$

$\begin{array}{ll}\text { Appendix } & 146\end{array}$

11. Appendix A:MPI parallelization of the combined TTM-MD model 147

11.1 Brief review of the combined TTM-MD model 147

11.2 MPI parllelization of the combined TTM-MD model 148

11.2.1 MPI parallelization of the MD part 151

11.2.2 MPI parallelization of the TTM part 158

11.2.3 Combination of TTM parallelization and MD parallelization $\quad 159$

11.2.4 General structure of of the parallel algorithm for the TTM-MD model 160

11.3 Performance of MPI parallelization of the combined TTM-MD model 163

12. Appendix B:Detailed analysis of structure of the runaway lattice-mismatched $\begin{array}{ll}\text { interface } & 166\end{array}$

13. Appendix C: Analytical derivation on nano-structuring equations 173

$\begin{array}{ll}\text { References } & 181\end{array}$ 


\section{LIST OF TABLES}

Table 1: Threshold fluences of melting, swelling and material removal from experiments and small-scale TTM-MD simulations. Both incident and adsorbed fluences are given, which can be converted using the Fresnel reflectivity, $R=0.969$, for the short pulse laser (with wavelength 800 $\mathrm{nm}$ ) irradiation on Ag.

Table 2: Stacking sequence of close-packed planes in the lattice of HCP, FCC, FCC with a stacking fault and FCC with a twin.

Table 3: The values of the diffusion barrier $E_{d}^{0}$ and scaling factor $\gamma$ in Eq. (2) obtained in quasistatic calculations and MD simulations (shaded cells) performed for different values of $\sigma_{a-s}$ and uniaxial lateral strain $e_{x x}$ ranging from -0.04 to 0.04 . 


\section{LIST OF FIGURES}

Figure 2.1: Four stages of the relaxation of optically excited electrons in a metal target irradiated by a short laser pulse. Schematic drawing is based on the discussion of Ref. [46].

Figure 2.2: Schematics of the TTM-MD model for simulation of laser interaction with a metal target. The laser irradiation is directed from the top of the figure.

Figure 3.1: Schematic sketch of the simulation setup. The $30 \mathrm{~nm} \mathrm{Ag} \mathrm{film} \mathrm{and} \mathrm{a} 200 \mathrm{~nm}$ part of the $\mathrm{Cu}$ substrate are represented with atomic-level resolution, using the TTM-MD model, whereas the electron heat conduction in the deeper part of the substrate is simulated with the conventional TTM.

Figure 3.2: Initial configuration of the $\mathrm{Ag}-\mathrm{Cu}$ interface equilibrated at $300 \mathrm{~K}$. Two atomic layers adjacent to the interface are shown, with atoms colored by their type (red for $\mathrm{Cu}$ and green for $\mathrm{Ag}$ ) in (a) and based on the output of a modified version [79] of the structural analysis suggested in Ref. [80] (light blue and green atoms belong to atomic configurations that correspond to FCC and HCP structures, respectively, the structure around the yellow atoms cannot be assigned to any crystal structure) in (b). The lines of misfit dislocations are shown by black arrows with small blue arrows indicating the corresponding Burgers vectors, $\vec{b}_{1}=\frac{1}{2}[110]$ and $\vec{b}_{2}=\frac{1}{2}[\overline{1} 10]$.

Figure 3.3: Contour plots of the lattice temperature (a), pressure (b), and density (c) for a simulation of a $\mathrm{Ag}-\mathrm{Cu}$ target irradiated with a $200 \mathrm{fs}$ laser pulse at an absorbed fluence of 0.13 $\mathrm{J} / \mathrm{cm}^{2}$. The laser pulse is directed along the Y-axis, from the top of the contour plots. The black lines separate the melted regions from the crystalline parts of the target. The red lines separate the atomistic (TTM-MD) and continuum (TTM) parts of the computational domain. The red rectangle in (a) shows the region and time interval for which snapshots are shown in Fig. 3.4. The density scale in (c) is normalized to the density of substrate $\mathrm{Cu}$ before the irradiation (at 300 $\mathrm{K}), \rho_{0}$. The fine "fingerprint"-type pattern observed in the crystalline parts of the target in the density plot is an artifact of the data analysis method - the physical properties are averaged over 
$1 \mathrm{~nm}$ thick slices and the stepwise variation of the number of atoms (and density) in each slice corresponds to an atomic layer entering/leaving the slice due to the material displacement in response to the pressure waves and thermal expansion

Figure 3.4: Time dependence of the lattice temperature of the interfacial region of a $\mathrm{Ag}-\mathrm{Cu}$ target irradiated with a $200 \mathrm{fs}$ laser pulse at an absorbed fluence of $0.13 \mathrm{~J} / \mathrm{cm}^{2}$. The horizontal dashed lines show the equilibrium melting temperatures of the FBD EAM Cu and Ag materials. The lattice temperature is calculated from the average kinetic energy of atoms in a $5 \mathrm{~nm}$ layer adjacent to the interface. The gray area marks the time interval from 840 to $960 \mathrm{ps,} \mathrm{during}$ which a resolidification front propagates through the region of atomic mixing from the side of the Ag film and meets another resolidification front that propagates from the side of the $\mathrm{Cu}$ substrate (see Fig. 3.5). The release of the latent heat of melting upon solidification of the interfacial region is responsible for the transient temperature increase during the time of the solidification.

Figure 3.5: Snapshots of the evolution of the atomic structure in the region of $\mathrm{Ag}-\mathrm{Cu}$ interface in the simulation illustrated by the contour plots in Fig. 3.3. The snapshots are taken for the region and the time interval marked in Fig. 3.3a by the red rectangle. Atoms are colored according to their type - $\mathrm{Ag}$ atoms are colored red and $\mathrm{Cu}$ atoms are colored blue. To reduce the thermal noise in atomic positions, the configurations are quenched for $0.2 \mathrm{ps}$ using a velocity dampening technique (see text). The fast quenching does not introduces any structural changes to the atomic configurations but makes the visual analysis more straightforward. The vertical white lines in each snapshot show the approximate locations of resolidification fronts propagating from the $\mathrm{Ag}$ (left) and $\mathrm{Cu}$ (right) sides of the system and meeting each other at $~ 955$ ps.

Figure 3.6: Concentration profiles (a), number of atoms in atomic planes (b), spacing between $\{200\}$ planes of the original FCC structure (c), and ratio c/a of the BCT lattice constants (d) shown for a region adjacent to the $\mathrm{Ag}-\mathrm{Cu}$ interface. The analysis is performed for an atomic configuration obtained by the end of the simulation (1.59 ns after the laser pulse) and quenched by applying the velocity dampening technique for $0.2 \mathrm{ps}$ (the snapshots of the corresponding atomic configuration are shown in Fig. 3.7(a-c). The definitions of the structural parameters 
shown in the plots are illustrated in (e). Each point in the plots is calculated for an individual atomic plane parallel to the surface of the layered system. The atomic planes correspond to (002) planes in the original FCC structures of the $\mathrm{Cu}$ substrate and the Ag film. In (c), $\mathrm{d}_{\mathrm{xy}}$ corresponds to the average spacing between the neighboring atomic planes that correspond to (200) and (020) planes in the FCC structure, $d_{z}$ corresponds to the average spacing between the neighboring (002) planes of the FCC structure, and atoms that do not belong to FCC or BCC/BCT atomic configurations are excluded from the averaging. In (d), the ratio c/a is defined as shown in (e), so that the perfect FCC and BCC structures are treated as general BCT structures with ideal ratio of $\mathrm{c} / \mathrm{a}=\sqrt{2}$ and 1 , respectively. The gray, green and yellow areas in the plots mark the regions of atomic mixing, pseudomorphic epitaxial layer of $\mathrm{Cu}$ on $\mathrm{Ag}$, and corrugated lattice-mismatched interface consisting of stacking fault pyramids illustrated in Fig. 3.8, respectively.

Figure 3.7: Snapshots of the atomic configurations in a region adjacent to the $\mathrm{Ag}-\mathrm{Cu}$ interface. The atomic configuration shown in (a-c) is obtained by the end of the simulation (1.59 ns after the laser pulse) and quenched by applying the velocity dampening technique for $0.2 \mathrm{ps.} \mathrm{The}$ atomic configuration shown in (d) and (e) is obtained by a gradual cooling ( $5 \mathrm{~K} / \mathrm{ps})$ of the system shown in (a-c). In (a), atoms are colored by their type: red for $\mathrm{Ag}$ and blue for $\mathrm{Cu}$. In (b-e), atoms are colored according to the output of a modified version [79] of the structural analysis of Ref. [80]: Light blue, green and blue atoms belong to local atomic configurations with FCC, $\mathrm{HCP}$ and $\mathrm{BCC} / \mathrm{BCT}$ structures, respectively; the structure around the yellow atoms cannot be assigned to any crystal structure and is characteristic of point defects and dislocation cores. The FCC atoms are blanked in (c) and (e) to expose the defect structure. The region shown in (a-c) corresponds to the region for which the distributions of structural and physical characteristics are shown in Fig. 3.6. The snapshots shown in (d) and (e) are aligned with the ones in (a-c) to match the location of the lattice-mismatched interface.

Figure 3.8: Structure of the "runaway" lattice-mismatched interface generated in the Ag-Cu system irradiated by a $200 \mathrm{fs}$ laser pulse. The interface is located in the $\mathrm{Cu}$ substrate, below the $\mathrm{Ag}-\mathrm{Cu}$ mixing region and the view from the side of the $\mathrm{Cu}$ substrate is shown in (a). Similarly to Fig. 3.7c, atoms are colored according to the local atomic structure, with green atoms located within the planes of intrinsic stacking faults in the FCC structure (local HCP configurations), yellow atoms cannot be assigned to any crystal structure and belong to the dislocation cores, and 
FCC atoms are blanked to expose the structure of the interface. The structure of the interface is represented by an array of stacking fault pyramids outlined by stair-rod partial dislocations. The lines of stair-rod partial dislocations in the base and at the edges of the pyramids (Lomer-Cottrell and Hirth locks, respectively) are shown by blue and red arrows. Examples of the dislocation reactions responsible for the formation of the locks are shown in the figure. A schematic representation of a structural element of the interfacial structure is shown in (b).

Figure 4.1: Schematic sketch of the simulation setups used in the exploration of the generation of BCC Cu by short pulse laser processing of: (a) $20 \mathrm{~nm} \mathrm{Cu}$ film - Ag substrate system. (b) $5 \mathrm{~nm}$ Ag film $-5 \mathrm{~nm} \mathrm{Cu}$ film - Ag substrate system. TTM-MD model is applied in the surface part of the target, whereas the electron heat conduction in the deeper part of the substrate is simulated with the conventional TTM. The desired melting region is marked by dashed squares.

Figure 4.2: Contour plots of the lattice temperature, pressure and density (normalized to the density of substrate $\mathrm{Ag}$ at $300 \mathrm{~K}$ before the irradiation) as a function of time and depth for simulations of $\mathrm{Cu}$ film - Ag substrate systems irradiated with $200 \mathrm{fs}$ laser pulses at fluences of (a) $0.03 \mathrm{~J} / \mathrm{cm}^{2}$, (b) $0.04 \mathrm{~J} / \mathrm{cm}^{2}$ and (c) $0.05 \mathrm{~J} / \mathrm{cm}^{2}$. Laser pulse is directed along the Y-axis, from the top of the contour plots. The black lines separate the melted regions from the crystalline bulk of the target. Purple dashed lines indicate the $\mathrm{Cu}-\mathrm{Ag}$ interface. Red lines separate the MD and continuum parts of the combined TTM-MD model. The $\mathrm{Cu}$ film is spallated at the laser fluence of $0.05 \mathrm{~J} / \mathrm{cm}^{2}$ as seen in c). The density scale in (c) is normalized to the density of substrate Ag before the irradiation (at $300 \mathrm{~K}$ ), $\rho_{0}$.

Figure 4.3: Contour plots describing the evolution of (a) lattice temperature a), (b) pressure, (c) density and (d) liquid fraction in the simulation of $\mathrm{Cu}$ film - Ag substrate systems irradiated with 200 fs laser pulse at absorbed fluences $\mathrm{F}=0.04 \mathrm{~J} / \mathrm{cm}^{2}$. The black lines separate the melted regions from the crystalline bulk of the target, while the red lines separate the MD and continuum parts of the combined TTM-MD model. The purple dashed lines mark the $\mathrm{Cu}-\mathrm{Ag}$ interface. The red rectangle and the blue circle in (c) show the region and time interval for which snapshots are shown in Fig. 4.4 and Fig. 4.5. The liquid fraction is obtained based on the calculation of local order parameter [63] as discussed in the text. 
Figure 4.4: Snapshots taken for the region and the time interval marked in Fig. 4.3c by the red rectangle. Atoms are colored according to their type in (a) - Ag atoms are colored blue and $\mathrm{Cu}$ atoms are colored red. Atoms are colored by their local structural environment in (b), where a snapshot for the time of $260 \mathrm{ps}$ is shown. To reduce the thermal noise, the atomic configuration is quenched for $0.2 \mathrm{ps}$ before the structural analysis is performed to obtain (b).

Figure 4.5: Snapshots taken for the region and the time interval marked in Fig. $4.3 \mathrm{c}$ by the blue circle. Atoms are colored according to their local structural environment. To reduce the thermal noise, the system is quenched for 0.2 ps before the structure analysis.

Figure 4.6: The results of a simulation of $200 \mathrm{fs}$ laser irradiation of a $15 \mathrm{~nm} \mathrm{Ag} \mathrm{film} \mathrm{-} 20 \mathrm{~nm} \mathrm{Cu}$ film - Ag (001) substrate system at a fluence of $0.06 \mathrm{~J} / \mathrm{cm}^{2}$. (a) Contour plots of the lattice temperature, pressure, and density as a function of time and depth, with black lines separating the melted regions from the crystalline bulk of the target and red lines separating the MD and continuum parts of the combined TTM-MD model. (b) A snapshot taken for the region marked by the blue rectangle in the density contour plot at a time of $35 \mathrm{ps}$. Spallation at the $\mathrm{Cu}-\mathrm{Ag}$ interface takes place with only intermediate $\mathrm{Cu}$ film melted.

Figure 4.7: Contour plots of the lattice temperature, pressure and density as a function of time and depth for simulations of $5 \mathrm{~nm} \mathrm{Ag} \mathrm{film} \mathrm{-} 5 \mathrm{~nm} \mathrm{Cu}$ film - Ag (001) substrate systems irradiated with 200 fs laser pulses at absorbed fluences of (a) $0.04 \mathrm{~J} / \mathrm{cm}^{2}$, (b) $0.05 \mathrm{~J} / \mathrm{cm}^{2}$, (c) $0.055 \mathrm{~J} / \mathrm{cm}^{2}$ and (d) $0.06 \mathrm{~J} / \mathrm{cm}^{2}$. Laser pulse is directed along the $\mathrm{Y}$-axis, from the top of the contour plots. The black lines separate the melted regions from the crystalline bulk of the target. Red lines separate the MD and continuum parts of the combined TTM-MD model. The two films are spallated at the laser fluence $0.06 \mathrm{~J} / \mathrm{cm}^{2}$ as seen in $(\mathrm{d})$.

Figure 4.8: Snapshots taken for the region and the time interval marked in Fig. 4.3c by the blue rectangle. Atoms are colored according to their type.

Figure 4.9: (a) Contour plot of density showing the resolidification process after 200 fs laser irradiation of $5 \mathrm{~nm} \mathrm{Ag} \mathrm{film}-5 \mathrm{~nm} \mathrm{Cu}$ film - Ag (001) substrate target at a fluence of $0.05 \mathrm{~J} / \mathrm{cm}^{2}$. The resolidification process can be clearly seen by the slopes of the black lines, which separate the melted regions from the crystalline bulk of the target. (b) Snapshots taken for the top surface 
region $(\sim 20 \mathrm{~nm})$ in the time interval from 100 ps to 460 ps. Atoms are colored by their type, with red color used for $\mathrm{Cu}$ and blue color for Ag. (c) Snapshot at $460 \mathrm{ps}$ with atoms colored by their local structural environment. To reduce the thermal noise, the system is quenched for 0.2 ps before the structural analysis.

Figure 4.10: (a) Contour plot describing lattice temperature evolution during TTM-MD simulation of $200 \mathrm{fs}$ laser irradiation of $5 \mathrm{~nm} \mathrm{Ag} \mathrm{film}-5 \mathrm{~nm} \mathrm{Cu}$ film - Ag (001) substrate target at a fluence of $0.05 \mathrm{~J} / \mathrm{cm}^{2}$. The cooling of the system down to $\sim 400 \mathrm{~K}$ during the $5 \mathrm{~ns}$ of the simulation can be seen from the contour plot. (b)-(d) Snapshots of the top surface region $(\sim 15$ $\mathrm{nm}$ ) viewed from [110] direction of the original FCC structure. Atoms are colored by their type, with red color used for $\mathrm{Cu}$ and blue color used for $\mathrm{Ag}$. (b) is taken at the time $460 \mathrm{ps}$ after the laser irradiation. (c) is taken at 4920 ps after the laser irradiation, when the system has uniform temperature of $\sim 420 \mathrm{~K}$. (d) is taken after the system is equilibrated at $300 \mathrm{~K}$ for $200 \mathrm{ps}$, and (e) is taken after the system is cooled down to $0 \mathrm{~K}$.

Figure 5.1: Setup for UHV laser ablation experiments. The laser beam is focused onto the sample using a $\mathrm{f}=250 \mathrm{~mm}$ achromatic lens. This figure is adopted from Ref. [20].

Figure 5.2: Series of SEM images of the ablation spots on $\mathrm{Ag}(001)$ surfaces irradiated by $100 \mathrm{fs}$ laser pulses at incident fluences from $2.45 \mathrm{~J} / \mathrm{cm}^{2}$ to $42.6 \mathrm{~J} / \mathrm{cm}^{2}$ : (a) $2.45 \pm 0.06 \mathrm{~J} / \mathrm{cm}^{2}$, (b) $3.90 \pm 0.07 \mathrm{~J} / \mathrm{cm}^{2}$, (c) $5.70 \pm 0.08 \mathrm{~J} / \mathrm{cm}^{2}$, (d) $9.40 \pm 0.10 \mathrm{~J} / \mathrm{cm}^{2}$, (e) $15.10 \pm 0.20 \mathrm{~J} / \mathrm{cm}^{2}$, (f) $24.1 \pm 0.3$ $\mathrm{J} / \mathrm{cm}^{2},(\mathrm{~g}) 34.9 \pm 0.4 \mathrm{~J} / \mathrm{cm}^{2}$ and (h) $42.6 \pm 0.5 \mathrm{~J} / \mathrm{cm}^{2}$. The white scale bar in each image corresponds to $20 \mu \mathrm{m}$. This figure is adopted from Ref. [20].

Figure 5.3: (a) An example of AFM surface scan of an ablation spot at incident laser fluence of $4.87 \pm 0.08 \mathrm{~J} / \mathrm{cm}^{2}$. The black horizontal lines mark the area from which the average line out is computed as shown in (b). (b) AFM line outs of several ablation spots on Ag (001) surface generated by irradiation at incident fluences from $3.17 \mathrm{~J} / \mathrm{cm}^{2}$ (absorbed fluence of $0.098 \mathrm{~J} / \mathrm{cm}^{2}$ ) to $42.6 \mathrm{~J} / \mathrm{cm}^{2}$ (adsorbed fluence of $1.321 \mathrm{~J} / \mathrm{cm}^{2}$ ). For clarity, the curves are offset vertically. The bumps are formed on the surfaces and the material is removed down to the levels below the initial surface level at the highest fluence. This figure is adopted from Ref. [20]. 
Figure 5.4: AFM line outs of the ablation spots observed on Al (111) surface irradiated by 100 fs laser pulses at different incident fluences ranging from $0.79 \mathrm{~J} / \mathrm{cm}^{2}$ to $4.7 \mathrm{~J} / \mathrm{cm}^{2}$. For clarity, the curves are offset vertically. This figure is adopted from Ref. [19].

Figure 5.5: Schematic sketch of the simulation setup. TTM-MD model is applied in the surface region of the target, down to $\sim 300 \mathrm{~nm}$, whereas the electron heat conduction in the deeper part of the substrate, down to $4 \mu \mathrm{m}$, is simulated with the conventional TTM.

Figure 5.6: Contour plots of the lattice temperatureand pressure as a function of time and depth obtained in simulations of $100 \mathrm{fs}$ pulse laser irradiation of $\mathrm{Ag}(001)$ at fluences of (a) $0.05 \mathrm{~J} / \mathrm{cm}^{2}$, and (b) $0.07 \mathrm{~J} / \mathrm{cm}^{2}$. Laser pulse is directed along the $\mathrm{Y}$-axis, from the top of the contour plots. The black lines separate the melted regions from the crystalline bulk of the target. Red lines separate the MD and continuum parts of the combined TTM-MD model. The simulated system has the lateral size $L_{x}=L_{y}=2.47 \mathrm{~nm}$.

Figure 5.7: Contour plots of the lattice temperature, pressure and density (normalized to the density of $\mathrm{Ag}$ at $300 \mathrm{~K}$ before the irradiation) as a function of time and depth for simulations of $100 \mathrm{fs}$ pulse laser irradiation of $\mathrm{Ag}$ (001) at absorbed fluences of (a) $0.08 \mathrm{~J} / \mathrm{cm}^{2}$, and (b) 0.09 $\mathrm{J} / \mathrm{cm}^{2}$. Laser pulse is directed along the $\mathrm{Y}$-axis, from the top of the contour plots. The black lines separate the melted regions from the crystalline bulk of the target. Red lines separate the MD and continuum parts of the combined TTM-MD model. The simulated system has the lateral size $L_{x}=L_{y}=2.47 \mathrm{~nm}$.

Figure 5.8: Snapshots of the top $100 \mathrm{~nm}$ of the $\mathrm{Ag}$ (001) target irradiated by a 100 fs laser pulse at a fluence of $0.10 \mathrm{~J} / \mathrm{cm}^{2}$. The snapshots show the process of voids formation and subsequent ejection of a melted surface layer. from 60 ps to 260 ps at 20 ps time intervals. The computation cell has the lateral size $L_{x}=4.94 \mathrm{~nm}$ and $L_{y}=9.88 \mathrm{~nm}$.

Figure 5.9: Contour plots of the lattice temperature, pressure and density as a function of time and depth for simulations of $100 \mathrm{fs}$ pulse laser irradiation of $\mathrm{Ag}(001)$ at fluences of $0.10 \mathrm{~J} / \mathrm{cm}^{2}$. The simulations are performed for two systems of different lateral size: (a) $L_{x}=L_{y}=2.47 \mathrm{~nm}$ and (b) $L_{x} \sim 4.94 \mathrm{~nm}$ and $L_{y} \sim 9.88 \mathrm{~nm}$. 
Figure 5.10: Contour plots of the lattice temperature, pressure and density as a function of time and depth for simulations of $100 \mathrm{fs}$ pulse laser irradiation of $\mathrm{Ag}$ (001) at absorbed fluences of (a) $0.15 \mathrm{~J} / \mathrm{cm}^{2}$, (b) $0.20 \mathrm{~J} / \mathrm{cm}^{2}$, (c) $0.25 \mathrm{~J} / \mathrm{cm}^{2}$, and (d) $0.30 \mathrm{~J} / \mathrm{cm}^{2}$. The black lines separate the melted regions from the crystalline bulk of the target. Red lines separate the MD and continuum parts of the combined TTM-MD model. The simulated system has the lateral size $L_{x} \sim 4.94 \mathrm{~nm}$ and $L_{y} \sim 9.88 \mathrm{~nm}$.

Figure 5.11: (a) The dependence of the maximum depth of melting, void formation and phase explosion on laser fluence obtained in TTM-MD simulations. (b) A map of ablation mechanisms proposed based on the comparison between the TTM-MD simulations and experiments. The maximum depth of phase explosion in (a) is defined as the maximum depth that reaches the threshold temperature for phase explosion, $3500 \mathrm{~K}$. The possible spallation region in (b) is not directly obtained from simulations, but a rough estimation based on experiment results shown in Fig. 5.3b.

Figure 6.1: Contour plots of the lattice temperature, pressure, collective velocity in the direction normal to the surface, and density as a function of time after the laser pulse and depth under the initial surface for simulations of $100 \mathrm{fs}$ pulse laser irradiation of $\mathrm{Ag}$ (001) target at absorbed fluences of (a) $0.08 \mathrm{~J} / \mathrm{cm}^{2}$, (b) $0.085 \mathrm{~J} / \mathrm{cm}^{2}$, and (c) $0.09 \mathrm{~J} / \mathrm{cm}^{2}$. Laser pulse is directed along the Y-axis, from the top of the contour plots. The black lines separate the melted regions from the crystalline bulk of the target. Red lines separate the MD and continuum parts of the combined TTM-MD model. The simulated system has the lateral size $L_{x}=L_{y}=98.7 \mathrm{~nm}$.

Figure 6.2: Contour plots showing the evolution of (a) the lattice temperature, (b) pressure, (c) collective velocity in the direction normal to the surface, and (d) density normalized to the density of $\mathrm{Ag}$ at $300 \mathrm{~K}$ before the irradiation as a function of time and depth in the surface region $(<70 \mathrm{~nm})$ for simulations of $100 \mathrm{fs}$ pulse laser irradiation of Ag (001) target at an absorbed fluences of $0.085 \mathrm{~J} / \mathrm{cm}^{2}$. The black lines separate the "melted regions" from the crystalline bulk of the target. The blue rectangle in the temperature contour plot (a) marks the region of homogeneous nucleation of multiple crystallites in the surface region of the target. The black dashed line in (c) shows the time when the collective velocity drops down to 0 . The red arrow indicates the resolidification crossing the region of voids. 
Figure 6.3: Snapshots viewed from top, showing the void nucleation, growth, and coalescence. To identify voids, the computational system is divided into cubic cells of $0.4 \mathrm{~nm}$ and cells without atoms inside are found and plotted.

Figure 6.4: Number of voids (a) and the total volume of voids (b) as a function of time after the laser irradiation at a fluence of $0.085 \mathrm{~J} / \mathrm{cm}^{2}$. Voids are defined as clusters of connected empty cubic cells as shown in Fig. 6.3. Four stages of void evolution are indicated.

Figure 6.5: A series of snapshots of atomic structure in the surface region $(<80 \mathrm{~nm})$ illustrating the resolidification process in the simulation of $100 \mathrm{fs}$ pulse laser irradiation of $\mathrm{Ag}(001)$ target at a fluence of $0.085 \mathrm{~J} / \mathrm{cm}^{2}$. The snapshots are shown for the times from from $100 \mathrm{ps}$ to $1200 \mathrm{ps}$ with the time interval of $100 \mathrm{ps}$. To reduce the thermal noise in atomic positions, the configurations are quenched for $0.2 \mathrm{ps}$. The atoms are colored by their potential energy with the scale from $-2.85 \mathrm{eV}$ to $-2.64 \mathrm{eV}$. The blue atoms have lower energy, indicating the solid structure, while the green atoms have higher energy, indicating the liquid structure. The red atoms are at the free surface and have the highest energy due to the contribution of the surface energy. The resolidification front separating the liquid from crystal is clearly seen.

89

Figure 6.6: Snapshots from the simulation of 100 fs pulse laser irradiation of Ag (001) target at a fluence of $0.085 \mathrm{~J} / \mathrm{cm}^{2}$ shown from $1300 \mathrm{ps}$ to $2000 \mathrm{ps}$ to illustrate the homogeneous resolidification process and the generation of nano-crystalline surface structure. The atoms are colored by their potential energy as in Fig. 6.5. The grain boundaries separating different nanograins can be clearly identified as lines of high energy atoms.

Figure 6.7: Cross sections cut at the depth of $30 \mathrm{~nm}$ from the initial surface for the snapshots shown in Fig. 6.6 to illustrate the homogeneous resolidification process and the generation of nano-crystalline surface structure. The atoms are colored by their potential energy. The grain boundaries separating different nano-grains can be clearly identified as lines of high energy atoms.

Figure 6.8: (a) Atomic configuration at $2 \mathrm{~ns}$ with atoms colored by their local structural environment: light blue for FCC atoms, green for HCP atoms, blue for BCC atoms and yellow for defect atoms. (b) Cross section cut at the depth of $35 \mathrm{~nm}$ from the initial surface with atoms 
colored by their local structural environment. Yellow lines indicate the grain boundaries. (c) Atomic configuration at $2 \mathrm{~ns}$ with atoms colored by the angle between the normal to the closepacked planes in the corresponding grain and the [001] direction of the original FCC lattice. Blue atoms in the bottom part indicate that they belong to the region that experienced epitaxial resolidification. Red atoms belong to the grain boundaries. (d) The nano-grain size distribution indicating the nano-grains have sizes of several nanometers in radius and the $\langle 111\rangle$ pole figure indicating the random orientations of the nano-grains.

92

Figure 6.9: Different stacking of close-packed planes to form HCP lattice (left) and FCC lattice (right).

Figure 6.10: Change of FCC stacking sequence after crossing a twin and the corresponding change in the orientation of the FCC tetrahedron.

Figure 6.11: A small region of nano-crystalline structure generated in the simulation. Some of the twins and stacking faults are indicated in the figure.

Figure 6.12: Three examples of five-fold twinning observed in the structure of laser-generated nano-crystalline surface region of the irradiated Ag (001) target. (a) shows an isolated 5-fold twinning structure. (b) and (c) show the structure of connected five-fold twinning.

Figure 6.13: Formation of five-fold twinning structure: (a) micro(nano)-rods with five-fold symmetry adopted from Ref. [110], (b) nano-structure of five-fold symmetry obtained in our simulations.

Figure 6.14: Formation of five-fold twinning structure. (a) Decahedron and a SEM image of Ag decahedral nanoparticles from Ref. [105], and (b) Icosahedron and a SEM image of Ag icosahedral nanoparticle from Ref. [105].

Figure 6.15: Contour plots showing the evolution of (a) the lattice temperature, (b) pressure, (c) collective velocity in the direction normal to the surface and (c) density normalized to the density of $\mathrm{Ag}$ at $300 \mathrm{~K}$ before the irradiation as a function of time and depth in the surface region $(<80$ $\mathrm{nm}$ ) for the simulation of $100 \mathrm{fs}$ short pulse laser irradiation on Ag (001) target at a fluence of $0.09 \mathrm{~J} / \mathrm{cm}^{2}$. Areas where the density of the material is less than $10 \%$ of the initial density before 
the irradiation are not shown in the plots. The black lines separate the "melted regions" from the crystalline bulk of the target.

Figure 6.16: A series of snapshots for the simulation performed at a laser fluence of $0.09 \mathrm{~J} / \mathrm{cm}^{2}$. The void growth, bridge elongation and the final separation of the top surface layer are clearly seen.

Figure 6.17: Cross sections through the middle of the computational cell of the atomic configurations shown in Fig. 6.16. The cross sections are shown to illustrate the resolidification process, especially the one in the nanospike.

Figure 6.18: Contour plots of temperature (a) and density (b) focused on the region of the solidification of the elongated bridge. Some obvious homogeneous nucleation sites are marked in (a) by the cross signs and their growth shown by the black dashed arrows. A cross section through the middle of the nanospike with atoms colored by its local structural environment (the coloring convention is the same as in Figs. 6.8a and 6.8b is shown in (c).

Figure 6.19: Nucleation of five-fold twinning structure in the region outlined by the red circle in Fig. 6.18a. Atoms are colored by their local structural environments and the coloring is the same as in Fig. 6.8a. The "defect" (liquid) atoms are blanked to clearly illustrate the nucleation process.

Figure 6.20: A series of cross sections of the nanospike made to illustrate the growth of five-fold twinning structure in the region outlined by the red rectangle in Fig. 6.18a. Atoms are colored by their local structural environments and the same coloring convention and scale as in Fig. 6.8a are used. Surface atoms are also blanked in the figures.

Figure 6.21: (a) A snapshot to illustrate the five-fold twinning structure: 6 inter-penetrating icosahedra truncated by the free surface of the nanospike. To clearly see the structure, surface atoms are blanked in (b), both surface atoms and FCC atoms are blanked in (c), and surface atoms, FCC atoms and HCP atoms are blanked in (d). All are the same snapshots with atoms colored by its local structural environment. 
Figure 6.22: Mapping the results of the simulations to the irradiation conditions of the experiment at the incident fluence of $3.17 \mathrm{~J} / \mathrm{cm}^{2}$ with the assumption that the laser energy deposition has a Gaussian energy profile and FWHM of $15 \mu \mathrm{m}$. (a) Laser fluence profile, (b) AFM line outs after laser irradiation, and (c) the proposed swelling mechanism.

Figure 6.23: Snapshots of atomic configurations generated in TTM-MD simulations of bulk Al irradiated by $100 \mathrm{fs}$ laser pulses at absorbed fluence $0.09 \mathrm{~J} / \mathrm{cm}^{2}$. Only top parts of the computational cells are shown in the snapshots. The atoms are colored by local density of their immediate surroundings.

Figure 7.1: (a) Schematic illustration of the transient grating method to create SAWs. Interference between the incoming excitation pulses results in a periodic oscillatory density of excited states, that excites a SAW propagating in z diretion. This figure is adopted from Ref. [121]. (b) Schematic illustration of the method of photoacoustic excitation of patterned surface to create SAWs. Nickel lines of width $L$ and period $p$ are patterned on a sapphire substrate. An 800 laser pulse heats the Ni creating a periodic stress that excites a SAW. This figure is adopted from Ref. [124].

Figure 7.2: Schematic representation of the computational system used in MD simulations of adatom diffusion on a substrate in the presence of a LISAW propagating along the $x$ direction. More details can be found in section 8 .

Figure 8.1: The computational system used in MD simulations of adatom diffusion on a substrate in the presence of a SAW propagating along the $x$ direction. The blue atoms in the region outlined by the red solid lines are displaced according to the analytical expressions for the desired Rayleigh wave. The purple atom is the adatom diffusing on the surface. The other atoms are colored by the instantaneous value of local strain. The atoms in the topmost 12 monolayers undergo unconstrained free dynamics and the atoms of the following 14 monolayers are coupled with a thermostat to keep a constant temperature of the system. The black dashed line marks the position of the free surface in the absence of SAW and the black arrow shows the wave propagation direction. 
Figure 8.2: Instantaneous strain and temperature distributions in a MD simulation performed for a running SAW with $e_{0}=0.048$ and $\lambda \approx 78 \sigma$. The average temperature $T_{0}$ of the surface is maintained close to $T_{t h}=0.15 \varepsilon / k_{B}$. To reduce thermal noise in the strain and temperature distributions, the results are averaged over 138 periods of wave propagation. The strain and temperature in the top surface monolayer are shows in (a) by blue delta and red square symbols, respectively. The contour plots in (b) and (d) show the strain and temperature distributions in the sub-surface region of the substrate. The black arrow in (b) shows the direction of the wave propagation.

Figure 8.3: The diffusion enhancement factor $D_{S A W} / D_{n}$ as a function of $\gamma_{e f f} e_{0}$ and $T_{0}$ obtained by numerical integration of Eq. (5). The inset shows the diffusion enhancement as a function of $\frac{\gamma_{e f f} e_{0}}{k_{B} T_{0}}$ predicted by Eqs. (5) and (6). The scale on the right side of the contour plot shows the values of the diffusion barrier $E_{d}^{0^{*}}=4 k_{B} T_{0} \ln 10$ that ensure an adatom jump rate of about $r^{*} \sim 0.1-1 \mathrm{~ns}^{-1}$ at the corresponding substrate temperature $T_{0}$. The white dashed line in the contour plot corresponds to $\gamma_{e f f} e_{0}=E_{d}^{0^{*}}$.

Figure 8.4: The diffusion enhancement $D_{S A W} / D_{n}$ by a SAW with $e_{0}=0.048$ as a function of $k_{B} T_{0} / E_{d}^{0}$ for adatoms with $\sigma_{a-s}=\sigma(\mathrm{a})$ and $\sigma_{a-s}=1.5 \sigma(\mathrm{b})$, respectively. The red solid line and the green dashed line show the predictions of Eqs. (5) and (6), respectively. The blue solid line and blue circles show the predictions of Eq. (3) and the results of kMC calculations, respectively. The large grey symbols show the results of MD simulations, with the error bars corresponding to the standard deviations of the mean calculated based on 8 independent series of the simulations.

Figure 8.5: Evaluation of the lifetimes of the excited vibrational state of adatom with $\sigma_{a-s}=\sigma$ (a) and $\sigma_{a-s}=1.5 \sigma(\mathrm{b})$. The lifetime is obtained by exponential fitting of the evolution of the amplitude of the oscillations of the kinetic energy an adatom initially placed at a saddle point position on a quenched substrate, as shown schematically in the inset in (a). Green lines show the results of the exponential fitting, with data from the initial parts of the simulations $\left(4 \tau_{0}\right.$ for 
$\sigma_{a-s}=\sigma$ and $54 \tau_{0}$ for $\left.\sigma_{a-s}=1.5 \sigma\right)$ used in the fitting. The long-term beatings of the kinetic energy are related to the interaction of weak acoustic pulses generated by the excited adatom with periodic boundary conditions used in the calculations (the substrate represented by $12 \times 12 \times 12$ FCC unit cells is used in these calculations).

Figure 9.1: Schematic illustration of the effect of surface strain generated by a SAW on the energy landscape for adatom diffusion. The dashed and solid lines show the energy barriers for adatom diffusion without and with the SAW, respectively.

Figure 9.2: Isochrone curves of the structuring time $\tau$ as functions of the substrate temperature $T_{0}$ and SAW wavelength $\lambda=2 \pi / k$ for representative values of $b=0.2 \mathrm{~nm}, R_{0}=1 \mathrm{ps}^{-1}$, and $E_{d}^{0}$ equal to 0.2 , and $0.4 \mathrm{eV}$, as predicted by the analytical model, Eq. (14). The solid, dashed and dash-dotted curves are for $\tau$ equal to $1 \mathrm{~ms}, 1 \mathrm{~s}$, and $1 \mathrm{~min}$, respectively.

Figure 9.3: Examples of SAW-induced gathering of adatoms in the regions of SAW-strain nodes (a) and anti-nodes (b). Density profiles are shown for different times given in units of characteristic structuring time $\tau$. Red solid curves show predictions of Eq. (14) and black symbols are the results of kMC simulations. (a) is for a system with $\gamma=0.91 \mathrm{eV}, \delta=0.30 \mathrm{eV}, \theta=$ $1.49, E_{d}^{0}=0.66 \mathrm{eV}, k_{B} T_{0} / E_{d}^{0}=0.1\left(T_{0}=770 \mathrm{~K}\right)$, i.e., $\gamma_{\text {eff }}\left(\gamma_{\text {eff }}-\delta\right) /\left(E_{d}^{0}\right)^{2}=6.9$ and (b) is for a system with $\gamma=0.43 \mathrm{eV}, \delta=1.95 \mathrm{eV}, \theta=1.49, E_{d}^{0}=0.16 \mathrm{eV}, k_{B} T_{0} / E_{d}^{0}=0.1\left(T_{0}=190 \mathrm{~K}\right)$, i.e., $\gamma_{\text {eff }}\left(\gamma_{\text {eff }}-\delta\right) /\left(E_{d}^{0}\right)^{2}=-33.5$. The same strain magnitude $e_{0}=0.04$ is used in both simulations. 139

Figure 11.1: Schematics of the TTM-MD model for simulation of laser interaction with a metal target. The laser irradiation is directed from the right side.

Figure 11.2: Schematics of the parallelization of the TTM-MD model for simulation of laser interaction with a metal target. The laser irradiation is directed from the right side. As an example, here the MD system is divided into 28 subdomains and the TTM system is divided into 8 subdomains. Each subdomain corresponds to a single computer core, and the total number of requested computer cores is 36 . Out of the total 36 cores, $28(0-27)$ cores, grouped into "MD_COMM_WORLD", are used for MD integration and the remaining 8 (28-35) cores ("TTM_COMM_WORLD”) are used to solve TTM equations. The red squares are the cells 
used for solving the TTM equations with a finite difference method. The red arrows indicate the communication between MD cores and TTM cores.

Figure 11.3: Spatial division of the MD computational cell into subdomains for parallelization. Each subdomain corresponds to a single computer core. Each subdomain is further divided into MD link cells (white squares). The TTM cell (red squares) used to calculate lattice temperature (for TTM) has the size of multiple times of a MD link cell. The division ensures an integer number of "TTM" cells in each subdomain. A single-link-cell border layer (green slashes) and a single-link-cell skin layer (purple slashes) are identified for each subdomain for the convenience of communications with neighboring subdomains and are shown for the subdomain 10 as an example.

Figure 11.4: Algorithm for implementation of Nordsieck fifth-order predictor-corrector MD integrator to achieve MPI parallelization with the overlap of communication and computation for a system with EAM potential. For the systems with pair potentials (e.g., L-J), the steps outlined by the black dot dashed lines are not needed.

Figure 11.5: Algorithm to achieve MPI parallelization of TTM part with the overlap of communication and computation.

Figure 11.6: Communication between MD and TTM cores used for synchronization of the TTM and MD parts of the model.

Figure 11.7: General structure for the parallel algorithm implementing the TTM-MD model.

Figure 11.8: The results of test runs performed on the Kraken cluster for a system consisting of 800,000 $\mathrm{Al}$ atoms. The $\mathrm{CPU}$ time per core is normalized to 1 in the simulation performed on a single core. A perfect scalability is observed up to 204 cores, down to $\sim 4000$ atoms per core.

Figure 11.9: Weak scaling behavior tested in simulations of 84 million Ag atoms on 6,016 cores, 168 million Ag atoms on 12,032 cores, and 336 million Ag atoms on 24,064 cores. The MD time step is $1 \mathrm{fs}$ in the simulations. 
Figure 11.10: Strong scaling behavior tested in simulations of a system of 336 million $\mathrm{Ag}$ atoms performed 1504, 3008, 6016, 12032, and 24064 cores. The time to solution is shown in (a) and the corresponding strong scaling speedup is shown in (b).

Figure 12.1: Two-dimensional representation of the Thompson tetrahedron illustrating the possible slip planes and the Burgers vectors of dislocations in an FCC crystal.

166

Figure 12.2: Dislocation reactions involved in the formation of the three-dimensional latticemismatched interface. (a) Square dislocation networks of the two-dimension semi-coherent interface. (b) The first step: dissociation into two perfect dislocations. (c) The second step dissociation into two partial dislocations. (d) The reaction of the partial dislocations to form the three-dimensional dislocation network of the new lattice-mismatched interface shown in (e).

Figure 13.1: Schematic illustration of the effect of surface strain generated by a SAW on the energy landscape for adatom diffusion. The dashed and solid lines show the energy barriers for adatom diffusion without and with the SAW, respectively. This figure is same as Fig. 9.1. 174 


\section{ACKNOWLEDGEMENTS}

First and foremost, I would like thank my advisor Prof. Leonid V. Zhiglei for his unconditional support and outstanding mentorship throughout my graduate study. Numerous fruitful and valuable discussions with him had a tremendous impact on completing this thesis. Thanks, Leo! I really enjoy working with you.

Second, I want to thank Prof. Vladimir Zaitsev for the stimulating discussions, comments, and advice. His help is indispensable to complete this thesis. I am impressed not only by his theoretical derivations, but also by his Russian music. Thanks, Vlad! I am looking forward to seeing you soon.

Third, I would like to thank our collaborators at the Aarhus University in Denmark, Prof. Peter Balling, Martin Snogdahl Christensen, and Juha-Matti Savolainen, for the stimulating discussions and valuable experimental results.

Fourth, I am also grateful to other members of my dissertation committee, Prof. Joseph Poon, Prof. Eugene Kolomeisky, Prof. Petra Reinke, Prof. James M. Howe, and Prof. Giovanni Zangari, for your precious time.

Fifth, I want to give special thanks to Dr. Alexey Volkov for his professional advice on the MPI parallelization of the TTM-MD code and his maintenance service in the computer clusters. Big thanks also go to all other members in Computational Material Group, Dr. Zhibin Lin, Maxim Shugaev, Eaman Tahir Abdul Karim, Cheng-Yu Shih, Richard Salaway, Bernard Wittmaack, Marshall Tabetah, Andrew Bennett-Jackson, and Ran Yang. They are not only good team workers, but also good friends in life.

Sixth, I appreciate the unconditional moral support from my parents, Guozhu Wu and Jiuai Han (Anqing, Anhui, P. R. China), my mother-in-law, Weilan Wang, and my brother Chengpeng $\mathrm{Wu}$.

Finally, I want to give the greatest thanks to my wife, Mengdi Chu, for her selfless love and support, and to my little daughter, Chloe Chuhanxi Wu, for her beautiful smiling. Mengdi and Chloe, I cherish the life with your accompany very much. 


\section{LIST OF PUBLICATIONS}

1) C. Wu, D. A. Thomas, Z. Lin, and L. V. Zhigilei, Runaway lattice-mismatched interface in an atomistic simulation of femtosecond laser irradiation of $\mathrm{Ag}$ film - $\mathrm{Cu}$ substrate system, Appl. Phys. A 104, 781-792 (2011)

(Section 3)

2) C. Wu, V. Yu. Zaitsev, and L. V. Zhigilei, Acoustic enhancement of surface diffusion, $J$. Phys. Chem. C 117, 9252-9258 (2013)

(Section 8)

3) C. Wu, E. T. Karim, A. N. Volkov, and L. V. Zhiglei, Atomistic movies of laser-induced structural and phase transformations from molecular dynamics simulation, Chapter for Lasers in Materials Science, Springer Series in Materials Science (Springer Verlag: New York, 2013), in press

(Part I)

4) C. Wu, V. Yu. Zaitsev, and L. V. Zhigilei, Mechanism of acoustically induced diffusional structuring of surface adatoms, Submitted to Phys. Rev. Lett. (2013)

(Section 9)

\section{Publications to be submitted:}

1) C. Wu, and L. V. Zhigilei, Generation of metastable BCC Cu structure in short pulse laser processing of $\mathrm{Ag}-\mathrm{Cu}$ multilayer targets: Atomic-level simulations, in final preparation

(Section 4)

2) C. Wu, and L. V. Zhigilei, M. S. Christensen, J. -M. Savolainen, and P. Balling, Atomistic modeling and experimental study of single-pulse femtosecond laser ablation, spallation, and damage of Ag targets, in final preparation

(Section 5 \& 6)

3) C. Wu, and L. V. Zhigilei, Computational study of hort pulse laser-induced surface microstructure modification, in final preparation

(Section 6)

4) C. Wu, V. Yu. Zaitsev, and L. V. Zhigilei, Spatial control of surface diffusion by surface acoustic waves, in final preparation

(Section 9)

5) C. Wu and L. V. Zhigilei, Molecular dynamics study of thermal and mechanical behavior of aluminum core/alumina shell nanoparticle under conditions of rapid heating, in final preparation

(Not related to this dissertation) 
6) C. Wu, E. T. Karim, and L. V. Zhiglei, Atomistic modeling of microstructure development in short pulse laser processing of metals, Chapter for Fundamentals of Laser-Assisted Micro- and Nanotechnologies, in preparation

(Part I)

\section{$\underline{\text { Additional publications not related to this dissertation: }}$}

1) C. H. Baker, $\underline{\text { C. Wu}}$, R. N. Salaway, L. V. Zhigilei, P. M. Norris, Vibrational contribution to thermal conductivity of silicon near solid-liquid transition, Proceedings of the ASME 2011 International Mechanical Engineering Congress \& Exposition, Paper no. IMECE2011-64064, (2011)

2) C. H. Baker, C. Wu, R. N. Salaway, L. V. Zhigilei, and P. M. Norris, Resolving the vibrational and electronic contributions to thermal conductivity of silicon near the solidliquid transition: Molecular dynamics study, Int. J. Transp. Phenom. 13, 143-150 (2013) 


\section{LIST OF PRSENTATIONS}

\section{(Underlined indicates presenter, italic indicates conference)}

\section{Oral Presentations:}

1) C. Wu, D. A. Thomas, Z. Lin, and L. V. Zhigilei, Atomic mixing and structural transformations in $\mathrm{Ag} / \mathrm{Au}$ film - $\mathrm{Cu}$ substrate systems irradiated by femtosecond laser pulses, COSIRES 2010, Krakow, Poland, July 2010 (Student travel grant awarded)

2) L. V. Zhigilei, Z. Lin, E. T. Karim, and C. Wu, Atomic-level simulations of laser interactions with metals: mechanisms of melting and resolidification, generation of crystal defects, COSIRES 2010, Krakow, Poland, July 2010

3) L. V. Zhiglei, Z. Lin, E. T. Karim, and C. Wu, Atomistic simulations of laser interactions with metals: melting of nanocrystalline films and generation of crystal defects, ICPEPA-7, Copenhagen, Denmark, August 2010

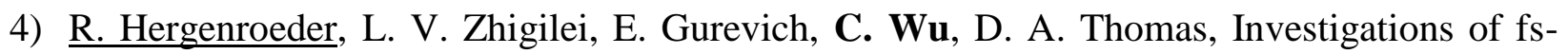
laser-induced phase transformations and atomic mixing in the $\mathrm{Au}$ film $-\mathrm{Cu}$ substrate system, ICPEPA-7, Copenhagen, Denmark, August 2010

5) C. Wu, and L. V. Zhigilei, Molecular dynamics study of thermal and mechanical behavior of aluminum core/alumina shell nanoparticles under conditions of rapid heating, SES 2010, Iowa State University, October 2010

6) L. V. Zhiglei, C. Wu, E. T. Karim, and Z. Lin, Generation of crystal defects and microstructure in laser processing of metals: molecular dynamics study, SES 2010, Iowa State University, October 2010

7) C. Wu, D. A. Thomas, Z. Lin, and L. V. Zhigilei, Atomic-level simulations of structural transformations in layered $\mathrm{Au}-\mathrm{Cu}$ and $\mathrm{Ag}-\mathrm{Cu}$ metal targets irradiated by a femtosecond lase pulse, APS, Dallas, Texas, March 2011

8) C. Wu, D. A. Thomas, Z. Lin, and L. V. Zhigilei, Molecular dynamics simulations of atomic mixing and structural transformations in layered $\mathrm{Au}-\mathrm{Cu}$ and $\mathrm{Ag}-\mathrm{Cu}$ metal targets irradiated by a femtosecond lase pulse, MRS, San Francisco, California, April 2011 
9) C. Wu, L. V. Zhigilei, M. S. Christensen, J-M. Savolainen, and P. Balling, Atomistic modeling and experimental study of single-pulse femtosecond laser ablation, spallation and damage of Ag and Al targets, ICPEPA-8, Rochester, New York, August 2012

10) C. Wu, V. Y. Zaitsev, M. Shugaev, and L. V. Zhiglei, Computational and theoretical analysis of acoustic enhancement of surface diffusion, AASP workshop, Breckenridge, Colorado, January 2013

11) V. Y. Zaitsev, C. Wu, and L. V. Zhiglei, Mechanism of averaged motion of diffusing adatoms in acoustic-strain field, AASP workshop, Breckenridge, Colorado, January 2013

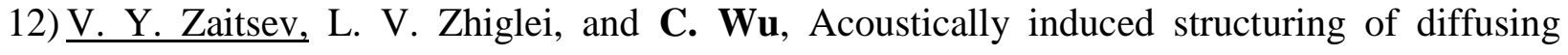
adatoms by standing surface waves, Nanoscale Pattern Formation at Surfaces, Copenhagen, Denmark, May 2013

\section{Poster presentations:}

1) C. Wu, L. V. Zhigilei, M. S. Christensen, J-M. Savolainen, and P. Balling, Atomistic modeling and experimental study of single-pulse femtosecond laser ablation, spallation and damage of Ag and Al targets, Cola 2011, Cancun, Mexico, November 2011

2) C. Wu, D. A. Thomas, Z. Lin, and L. V. Zhigilei, Generation of metastable BCC Cu in short pulse laser processing of multi-layer metal targets: atomic-scale simulations, Cola 2011, Cancun, Mexico, November 2011

3) C. Wu, V. Y. Zaitsev, M. Shugaev, and L. V. Zhiglei, Theoretical and computational study of nano-structuring under standing surface acoustic waves, AASP workshop, Breckenridge, Colorado, January 2013

4) C. Wu, V. Y. Zaitsev, M. Shugaev, and L. V. Zhigilei, Computational and theoretical analysis of acoustic enhancement of surface diffusion, AASP workshop, Breckenridge, Colorado, January 2013

5) M. Shugaev, V. Y. Zaitsev, C. Wu, and L. V. Zhiglei, Molecular dynamics investigation of nonlinear surface waves, AASP workshop, Breckenridge, Colorado, January 2013 


\begin{abstract}
Rapid progress in the development of accessible sources of short (pico- and femtosecond) laser pulses opens up new opportunities for surface modification with high accuracy and spatial resolution. In particular, the shallow depth of the heat-affected zone in short-pulse laser processing of strongly absorbing materials can result in the confinement of the laser-induced structural modifications within a surface layer as small as tens of nanometers. The small size of the laser-modified region and the highly non-equilibrium nature of the transient processes occurring in the laser-irradiated targets, however, make time-resolved experimental evaluation of laser-induced structural transformations challenging and hinder the interpretation of the experimental results. In this work, we use atomic-level computer simulations to investigate the microscopic mechanisms of the ultrafast structural and phase transformations in the metal targets induced by the short pulse laser irradiation. The simulations are performed with a computational model that combines classical molecular dynamics method with a continuum description of the laser excitation and subsequent relaxation of the conduction band electrons.

Three sets of simulations are performed to address a range of research questions related to short pulse laser processing. In the first set of simulations, we investigate the atomic mixing and structural modification of an interfacial region in a target composed of layers of metals that are immiscible in the solid state. Using the $\mathrm{Ag}-\mathrm{Cu}$ layered system as an example, we find that the region of atomic mixing generated by the fast melting and resolidification of the interface is substantially wider compared to the width of the equilibrium $\mathrm{Cu}-\mathrm{Ag}$ interface and have a pronounced asymmetric shape that reflects the preferential melting of the $\mathrm{Cu}$ substrate. Moreover, the generation of a "runaway" lattice-mismatched interface shifted into the $\mathrm{Cu}$ substrate separated from the $\mathrm{Ag}-\mathrm{Cu}$ mixing region by an intermediate pseudomorphic $\mathrm{BCC} \mathrm{Cu}$ layer is observed in the simulations. The detailed structural analysis reveals that the new latticemismatched interface has a complex three-dimensional corrugated structure consisting of a periodic array of stacking fault pyramids outlined by stair-rod partial dislocations. The material systems optimized for stabilization of the metastable $\mathrm{BCC} \mathrm{Cu}$ are explored in several targeted simulations and the experimental configuration for generation of a $5 \mathrm{~nm} \mathrm{BCC} \mathrm{Cu}$ layer sandwiched between two FCC- $\mathrm{Ag}(001) / \mathrm{BCC}-\mathrm{Cu}(001)$ interfaces is proposed based on the simulation results.
\end{abstract}


In the second set of the simulations, we investigate the processes responsible for an unexpected effect of surface "swelling" observed in recent experiments performed for $\mathrm{Al}$ and $\mathrm{Ag}$ targets irradiated by $100 \mathrm{fs}$ laser pulses. A series of simulations of short pulse laser irradiation of Ag targets are performed to investigate the mechanisms of void formation and spallation (separation of a melted layer from the bulk of the target) due to the short pulse laser-induced photomechanical processes. We find that at laser fluences just below the spallation threshold, the voids can be captured by fast resolidification. The computational prediction of the generation of porous structure of a surface region of the irradiated target provides an explanation for the surface swelling observed in the experiments. Besides the surface swelling, the largescale atomistic simulations reveal the generation of nanocrystalline structure of the surface layer of the irradiated target. The formation of the nanocrystalline structure is attributed to the homogeneous nucleation and growth of multiple randomly oriented crystallites under conditions of strong undercooling. The structural analysis on the nanocrystalline structure shows that the nano-grains have close-packed structure, with large number of stacking faults, twin boundaries and complex five-fold twinning structures. The grain boundaries, stacking faults, and twin boundaries are all likely to present strong barriers for dislocation propagation, resulting in the effective hardening of the surface region of the irradiated target.

Besides the direct modification of the surface region, transient thermoelastic stresses generated by short pulse laser irradiation can result in the emission of acoustic waves. The generation of photoacoustic waves has been widely used for material characterization, laser cleaning, and laser desorption. In the final part of this work, we combine theoretical analysis with molecular dynamics and kinetic Monte Carlo simulations to investigate the effect of laserinduced acoustic waves on surface diffusion. The substantial acoustic enhancement of surface diffusion (up to hundreds of percents) observed in the simulations suggests an attractive alternative to thermal activation in thin film growth on heat-sensitive substrates. It is found that, in addition to the diffusion enhancement, the surface self-structuring (spatial modulation of adatom concentration) can be effectively induced by standing surface acoustic waves. The predicted self-structuring phenomenon may open up opportunities for acoustic control of surface self-assembly without permanent modification of the substrate and growth conditions. 


\section{Introduction}

Ever since Theodore Maiman made the first laser in 1960 [1], the laser technology has developed rapidly with vastly improved laser performance, and tremendously increased laser variety. The ultrashort lasers are attracting special interest due to the high energy intensity it can achieve. In 1972, Erich Ippen and Charles Shank first generated laser pulses with duration of 1.5 ps [2]. In 1987, Richard Fork's group at Bell Lab generated 6 fs laser pulses [3]. A short, but rich history of laser development can be found in Ref. [4] with more details. The rapid development of laser technology has been accompanied by its broad applications in scientific research, consumer products, telecommunication, medicine, and materials processing. In particular, short (nanosecond to femtosecond) pulsed laser-based techniques have been widely used in laser surface processing (laser surface alloying, cladding, annealing, and hardening, e.g. [5-10]), laser cleaning [11-14], and laser desorption [15-18].

The extremely fast and localized energy deposition in short pulse laser irradiation leads to a cascade of coupled transient processes (superheating, melting, deformation, acoustic wave generation, material removal, etc.) occurring in a region of very small size (tens of nanometers) on a very short time scale (picoseconds to nanoseconds). This is a great advantage of pulsed laser-based techniques, since it allows materials processing of practically any material with extremely high precision and minimal collateral damage. However, the short time scale and the small size of the laser-modified region make the experimental characterization of laser-induced processes challenging. That is why the interaction of ultrafast laser pulses with materials is still not fully understood; though the short pulse laser-based techniques are widely used.

On the other hand, atomic-level MD modeling fits perfectly to the nature of short time scale and small spatial scale involved in short pulse laser irradiation, and is playing an increasingly important role in the development of the theoretical understanding of laser-materials interactions and the advancement of laser applications. In this thesis, the atomic-level computer modeling is first applied to study the short pulse laser-metal interaction, with a particular focus on the laser-induced surface structure modification. Then the non-thermal processes driven by laser-induced acoustic waves are explored by combining the atomistic modeling with theoretical analysis. 


\subsection{Part I: Short pulse laser interaction with metals}

Short pulse laser-material interaction is widely used in many modern processing and fabrication techniques, including laser surface alloying, cladding, annealing, hardening, e.g. Refs. [5-10]. Due to the extremely fast and localized energy deposition, short pulse laser irradiation brings the material in the surface region of the irradiated target to a state of strong electronic, thermal, and mechanical non-equilibrium and triggers a cascade of interrelated processes that may involve structural and phase transformations, such as melting and resolidification, generation of crystal defects, fracture and disintegration of the region of the laser energy deposition due to photomechanical stresses (front surface spallation), vaporization and explosive boiling of strongly overheated surface region. The laser-induced improvement of surface properties may be achieved by structural and compositional surface modifications through the formation of metastable crystalline and amorphous phases, grain refinement, generation/annealing of crystal defects. The need for computer modeling is amplified by its ability to provide clear visual representations, or "atomic movies," of laser-induced dynamic processes that naturally accounts for the complexity of the material response to the rapid laser energy deposition. In the part I of this thesis, a series of atomistic simulations are first performed on the $\mathrm{Ag}-\mathrm{Cu}$ layered targets to study the atomic mixing, interface structure, and structural transformations under short pulse laser irradiation, with a special effort devoted to the exploration of the formation of BCC Cu structure and its stability (see sections 3 and 4). Then, we perform a series of simulations of femtosecond laser irradiation of $\mathrm{Ag}$ (001) target (see sections 5 and 6), with the goal of explaining a recent experimental observation of surface swelling [19,20]. In addition, the surface microstructure after laser irradiation is carefully studied.

\subsection{Part II: short pulse laser-induced photoacoustic control of surface diffusion}

Due to the extremely fast energy deposition, short pulse laser irradiation of a strongly absorbing target not only results in a sharp temperature increase in the surface region, but, unavoidably, produces strong thermoelastic stresses in the region of the laser energy deposition. The relaxation of the strong thermoelastic stresses leads to the generation of acoustic pulses of a high intensity and frequency in the range of up to hundreds of GHz. Photoacoustic pulses 
produced via the short pulse laser irradiation were first observed in 1963 [21]. As the laser technology progresses, the laser unltrasonic techniques develop as well and are applied for surface acoustic waves (SAWs) generation and monitoring [22,23]. The generation of the photoacoustic pulses is used in pump-probe laser ultrasonics technique for measurements of film thickness and characterization of microstructure, e.g. [24,25]. The ability of the acoustic waves to desorb molecules, atoms and ions from surfaces has also been discussed in literature and supported by experimental evidence and theoretical calculations [15-18]. In dry laser cleaning, laser generated transient surface deformation is used for removal of small particles from the irradiated surfaces [11-14]. In the part II of this thesis, we first perform a series of atomistic simulations, combined with a theoretical model, to explore the possibility of promoting the surface diffusion of adsorbed species by laser-induced surface acoustic waves (LISAWs) (see section 8). The predicted surface diffusion enhancement suggests that the LISAWs may provide an attractive alternative to thermal activation in thin film growth on heat-sensitive substrates. Then, the effect of self-structuring (spatial modulation of adatom concentration) induced by standing LISAWs is investigated (see section 9). The predicted surface self-structuring suggest a possibility of nucleation control by standing LISAWs, which may be used to guide the process of island nucleation thin film growth. 
PART I: Short Pulse Laser Interaction with Metals 


\section{Introduction}

\subsection{Background}

Short (pico- and femtosecond) pulse laser interaction with metals has been widely used in many modern processing and fabrication techniques, including laser surface alloying, cladding, annealing, and hardening, e.g. [5-10]. Due to the extremely fast heating and cooling rates, short pulse laser irradiation has the ability to bring material into a sequence of nonequilibrium states, such as strong superheating and undercooling, high stress and sharp stress gradient. These highly nonequilibrium conditions can excite a cascade of coupled transient processes, such as fast melting and resolification, generation of defects and metasble phases, as well as material removal from the target. Taking advantage of the short pulse laser-induced nonequilibrium states and processes, the material surface properties can be improved through the formation of metastable crystalline and amorphous phases, grain refinement, generation/annealing of crystal defects, redistribution of the alloying elements, and changes in the phase segregation patterns.

The shallow depth of the heat-affected zone in short-pulse laser processing of strongly absorbing materials can result in the confinement of the laser-induced structural modifications within a surface layer as small as tens of nanometers, e.g. [26-30]. The small size of the lasermodified region makes experimental characterization of laser-induced structural changes challenging and, at the same time, increases the importance of understanding the generation, mobility, interactions and stability of individual crystal defects. At the same time, the fast and highly localized energy deposition in short pulse laser irradiation unavoidably creates the conditions of strong thermodynamic, electronic, and mechanical nonequilibrium, making the theoretical description of the structural transformations difficult. For multi-component and composite targets, an additional challenge is to provide an adequate description of the mass transfer and compositional changes in the transiently heated and melted surface region. The conventional analysis of the atomic diffusion and thermodynamic driving forces $[31,32]$ is hardly applicable to systems experiencing a very fast melting-resolidification cycle.

On the other hand, atomic-level computer modeling has been demonstrated to have the ability to provide detailed information on the complex structural and phase transformations induced by short pulse laser irradiation [33-44] and can assist in the advancement of laser-driven 
applications. In particular, recent atomistic simulations of laser interactions with metal targets provided insights into the mechanisms and driving forces responsible for the generation of point defects (vacancies and interstitials) in $\mathrm{Cr}$ targets [40], revealed the processes responsible for the formation of a nanocrystalline structure in regions of localized photoexcitation of metal films [41], clarified the effect of the grain boundaries on the kinetics of laser melting of nanocrystalline Au films [42], and helped to establish the connections between the maximum melting depth, photomechanical spallation, and phase explosion in short pulse laser interactions with metal targets [43]. First simulations of laser interactions with layered metal targets have also been reported for $\mathrm{Au}$ films deposited on a bulk $\mathrm{Cu}$ substrate [44]. The initial energy redistribution and the depth of the region undergoing melting and resolidification are found to be strongly affected by the difference in the strength of electron-phonon coupling of the film and substrate materials and by the transient variations of the electron-phonon coupling related to the thermal excitation of lower band electrons [45].

\subsection{Introduction to short pulse laser-metal interaction process: two temperature model}

Four stages can be distinguished for the response of a metal target to short pulse laser irradiation, as shown in Fig. 2.1. At the first stage of the laser excitation, the short pulse laser energy is adsorbed by conduction band electrons, leading to deviation of electron energy distribution from the equilibrium Fermi-Dirac distribution by exciting electrons located below the Fermi level to levels above the Fermi level. The second stage of electronic thermalization starts immediately after the laser excitation through the collisions of the excited electrons with those near the Fermi level. During the process of electronic thermalization, highly excited electrons can penetrate deep into the sample ballistically with about the Fermi velocity $\left(\sim 10^{6}\right.$ $\mathrm{m} / \mathrm{s}$ ). The occurrence of the ballistic energy transport has been confirmed by measurements of the transient reflectance of noble metal films [46,47]. The ballistic penetration depth is on the order of $100 \mathrm{~nm}$ for noble metals and on the order of optical penetration depth, 10-20 nm, for transition metals. The smaller ballistic range in transition metals can be intuitively understood by the high electron state density at the Fermi level, which cuts through the high-energy edge of the d-bands in transition metals [45]. Thus, the thermalization collision rate is high, which reduces the thermalization time and the ballistic penetration depth. The mean free path of electrons [48] can serve as a first estimate of the ballistic penetration depth. At the end of the 
second state, the local thermal equilibrium among electrons is established, i.e., Fermi-Dirac distributions with different electron temperatures at different depths are generated. The hot electrons are localized either within the ballistic range or within the optical penetration depth, where the lattice still keeps the initial low temperature, i.e, the strong non-equilibrium between the electrons and lattice is generated. The time needed for the second stage of electron thermalization is on the order of $\sim$ tens of femtoseconds. The third stage of electron-phonon thermalization consists of two competing processes: electron-phonon coupling and electronic thermal conduction. The electron-phonon coupling is driven by the strong non-equilibrium between electron and lattice temperature $\left(T_{e}>>T_{l}\right)$, and leads to energy transfer from electrons to lattice (phonons). The time ( tens of ps) needed for electron-phonon thermalization (the third stage) is defined by the strength of electron-phonon coupling. The electron-phonon coupling is generally stronger in transition metals than in noble metals and its dependence on the electron temperature has been investigated based on first-principles electronic structure calculations [45] in our group. Simultaneously with the electron-phonon equilibration process, the hot electrons transfer energy into the deeper parts of the target through the electronic thermal diffusion driven by the electronic temperature gradient generated at the second stage. The diffusion length for hot electrons before the establishment of the electron-phonon thermal equilibrium is defined by the electron-phonon thermalization time and also by the electronic thermal conductivity. In general, the electron-phonon thermalization time is tens of picoseconds to achieve the local electronphonon equilibrium. After the establishment of the electron-lattice thermal equilibrium, the fourth stage of common thermal diffusion sets in. Even though the local thermal equilibrium is generated among the electrons (stage 2) and between electrons and phonons (stage 3), there is still a temperature gradient in the surface region of the target, which drives the electronic thermal diffusion into the deeper part of the target. The interplay between the electronic thermal diffusion and the electron-phonon coupling results in the cooling of the target surface. 


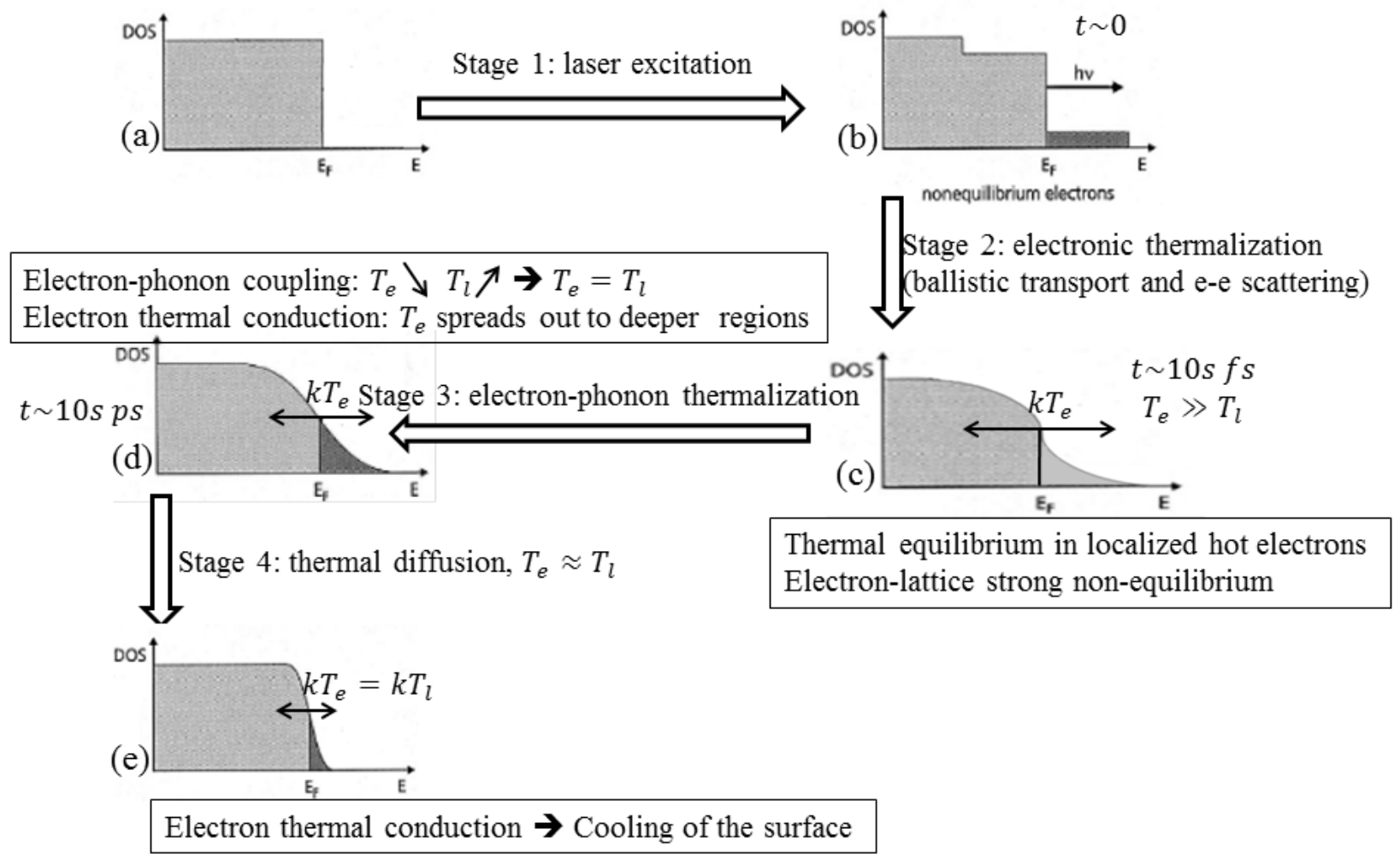

Figure 2.1: Four stages of the relaxation of optically excited electrons in a metal target irradiated by a short laser pulse. Schematic drawing is based on the discussion of Ref. [46].

As discussed above, the first two stages of laser excitation and electronic thermalization occur very fast, at the time scale of tens of femtoseconds. Assuming that electron thermalization take place instantaneously upon laser irradiation, the two-temperature model (TTM) proposed in 1974 [49] can be used to describe the short pulse laser-metal interaction by introducing two different temperatures, one for the lattice and the other for the electron. The accuracy of this assumption has been later justified by Rethfeld et al. [50], who solved a set of Boltzmann equations, which accounts for the non-equilibrium electron distribution. It was found that for short (pico- and femtosecond) pulse laser irradiation, the difference between the solutions of the Botlzman equations and TTM equations is negligible for sufficiently high laser fluence, e.g. near the threshold for laser melting or damage.

Essentially, TTM assumes that the initial electronic temperature distribution is established during the time of short pulse laser irradiation, and the temperature (electron and lattice) evolution is described by two coupled non-linear differential equations: 


$$
\begin{aligned}
& C_{e}\left(T_{e}\right) \frac{\partial T_{e}}{\partial t}=\nabla \cdot\left[K_{e}\left(T_{e}\right) \nabla T_{e}\right]-G\left(T_{e}\right)\left(T_{e}-T_{l}\right)+S(z, t) \\
& C_{l}\left(T_{l}\right) \frac{\partial T_{l}}{\partial t}=\nabla \cdot\left[K_{l}\left(T_{l}\right) \nabla T_{l}\right]+G\left(T_{e}\right)\left(T_{e}-T_{l}\right)
\end{aligned}
$$

The upper equation is for the electronic temperature $T_{e}$, and the lower one for the lattice temperature $T_{l}$. $G$ is the electron-phonon coupling factor. $C_{e}$ and $C_{l}$ denote the heat capacities of the electrons and lattice, respectively. Similarly, $K_{e}$ and $K_{l}$ are the electron and lattice thermal conductivities. The laser source term $S(z, t)$ only appears in the equation for the electron temperature, corresponding to the assumption of instantaneous electron thermalization. A Gaussian temporal profile is used in the simulations to describe the laser energy deposition, $S(z, t)=I_{0} L_{p}{ }^{-1} \exp \left(-z / L_{p}\right) \exp \left(-\left(t-t_{0}\right)^{2} / 2 \sigma^{2}\right)$, where $I_{0}$ is the peak intensity, $L_{p}$ is the optical adsorption depth, and $\sigma$ is the standard deviation of the Gaussian profile, related to the pulse duration as $\tau_{L}=$ (full width at half maximum) $=\sigma \sqrt{8 \ln (2)}$. The ballistic energy transport can be incorporated into the TTM model by substituting the optical penetration depth $L_{p}$ in the source term by an effective laser energy deposition depth defined by both optical adsorption and ballistic energy transport, $L_{p}+L_{b}$, where $L_{b}$ is the ballistic penetration depth. The electron-phonon coupling is described by the term $G\left(T_{e}\right)\left(T_{e}-T_{l}\right)$, which is present in both equations to make them coupled. The electronic thermal conduction is taken into account by the term $\nabla \cdot\left[K_{e}\left(T_{e}\right) \nabla T_{e}\right]$ in the equation of electronic temperature. The lattice thermal conduction $\nabla \cdot\left[K_{l}\left(T_{l}\right) \nabla T_{l}\right]$ can be removed as it is typically negligible compared to the electronic thermal conduction.

Even though TTM has been widely used in the computational studies of short-pulse lasermetal interactions, there is an intrinsic inability of this continuum model to fully describe the detailed kinetic process involved in short pulse laser-metal interaction, such as structural and phase transformations. In order to overcome this limitation, a combined atomistic-continuum model, so called TTM-MD model [34], has been developed, which combines the classical molecular dynamics (MD) method with the TTM model as discussed below. 


\subsection{The combined TTM-MD model [34]}

The goal of the combined TTM-MD model is to overcome the limitations and combine the advantages of traditional TTM and MD methods. Briefly, the TTM-MD model combines the classical MD method for simulation of non-equilibrium processes of lattice superheating and fast phase transformations induced by the laser energy deposition with a continuum description of the laser excitation and subsequent relaxation of the conduction band electrons, based on the twotemperature model (TTM) [49]. As schematically illustrated in Fig. 2.2, the MD method is combined with the TTM equation for $T_{e}$ in the very surface region of the target (TTM-MD region), in which MD substitutes the TTM equation for $T_{l}$ to capture the active processes of laser melting, void nucleation, and spallation/ablation, whereas TTM equation for $T_{e}$ accounts for the laser excitation, electron-phonon coupling and the dominant electronic thermal conduction. The diffusion equations for both electron $T_{e}$ and lattice $T_{l}$ temperatures are solved in the deeper region (TTM region) affected by the thermal conduction. The depth of the TTM region is chosen so that no significant increase in the electron or lattice temperatures is observed at the bottom during the time of the simulation.

A special pressure transmitting boundary condition [51,52] is used in this case to imitate the propagation of the laser induced stress wave from the MD region of the computational system to the continuum part of the model. The energy carried away by the stress wave is monitored to check energy conservation in the combined atomistic-continuum model [53]. Since the experimental laser spot size ( tens of micrometers) is typically much larger as compared to the lateral size of the computational cell (maximum of $~ 100$ nanometers), the periodic boundary conditions and spatial uniform laser intensity profile are applied in the lateral direction as shown in Fig. 2.2.

In the TTM-MD region, the cells (red squares) in the finite difference discretization are related to the corresponding volumes of the MD system and the local lattice temperature $T_{l}$. is calculated for each cell from the average kinetic energy of thermal motion of atoms in MD system. An additional force, $\xi m_{i} \vec{v}_{i}^{\text {th }}$, is added to the MD equations of motion to account for the

effect of electron-phonon coupling. In this coupling term, $m_{i}$ is the mass of an atom, $\vec{v}_{i}^{\text {th }}$ is the thermal velocity of an atom defined as $\vec{v}_{i}^{t h}=\vec{v}_{i}-\vec{v}_{i}^{c}$, where $\vec{v}_{i}$ is the actual velocity of an atom $i$ 
and $\vec{v}_{i}^{c}$ is the velocity of the center of mass of a cell to which atom $i$ belongs, and $\xi$ is a coefficient defined by the difference between the local lattice and electron temperatures, i.e., $\xi=\frac{G V_{N}\left(T_{e}-T_{l}\right)}{2 K^{T}}$, where $G$ is the electron-phonon coupling factor, $V_{N}$ is the cell volume, $K^{T}$ is the thermal kinetic energy of atoms in the cell. In fact, the additional force serves the same purpose as does the coupling term $G\left(T_{e}-T_{l}\right)$ in the TTM equation for lattice temperature $T_{l}$.

In practice, after each time-step of MD integration (MD time-step $\Delta t_{M D}$ ), the local lattice temperature $T_{l}$. is calculated for each cell in the TTM-MD region by averaging kinetic energy of thermal motion of atoms in the cell, i.e., $T_{l}=\frac{2 K^{T}}{3 N k_{B}}=\frac{\sum_{i=1}^{N} m_{i}\left(\vec{v}_{i}{ }^{t h}\right)^{2}}{3 k_{B} N}$, where the summation $\sum_{i=1}^{N}$ is performed over all $N$ atoms in the cell. The newly calculated lattice temperature $T_{l}$ enters as input to solve the TTM equation of electronic temperature $T_{e}$ (Eq.1 in Fig. 2.2). To stabilize the calculation, the time-step of the finite difference integration of TTM equation for electronic temperature $T_{e}$ (TTM time-step $\Delta t_{T T M}$ ) Eq.1 in Fig. 2.2) is typically $n$ times smaller than the timestep of MD integration, i.e., each MD time-step consists of $n$ TTM time-step $\Delta t_{M D}=n \Delta t_{T T M}$. " $n$ " can be estimated based on the Von Neumann stability criterion, $\Delta t_{T T M} \leq 0.5 \Delta x_{T T M}{ }^{2} C_{e} / K_{e}$, where $\Delta x_{T T M}$ is the TTM cell size. Thus, calculation of the coefficient $\xi=\frac{G V_{N}\left(T_{e}-T_{l}\right)}{2 K^{T}}$ in each MD time-step involves averaging over $n$ TTM time-steps that fits to the one MD time-step, i.e., $\xi=\frac{1}{n} \sum_{k=1}^{n} \frac{G V_{N}\left(T_{e}^{k}-T_{l}\right)}{2 K^{T}}=\frac{\frac{1}{\Delta t_{M D}} \sum_{k=1}^{n} \Delta t_{T T M} G V_{N}\left(T_{e}^{k}-T_{l}\right)}{2 K^{T}}=\frac{1}{\Delta t_{M D}} \frac{\Delta E^{e-p h}}{2 K^{T}}$, where $\Delta E^{e-p h}$ is the energy transferred from electrons to lattice through electron-phonon coupling, obtained during solving the TTM equation of electronic temperature $T_{e}$ over $n$ TTM time-steps. There is an underlying assumption that the lattice temperature keeps the same during $n$ TTM time-steps and is only updated every MD time-step. This is a reasonable assumption since the lattice heat capacity is several orders of magnitude higher than the electronic heat capacity, and the lattice temperature change during one TTM time-step is negligible compared with the electronic temperature change. 


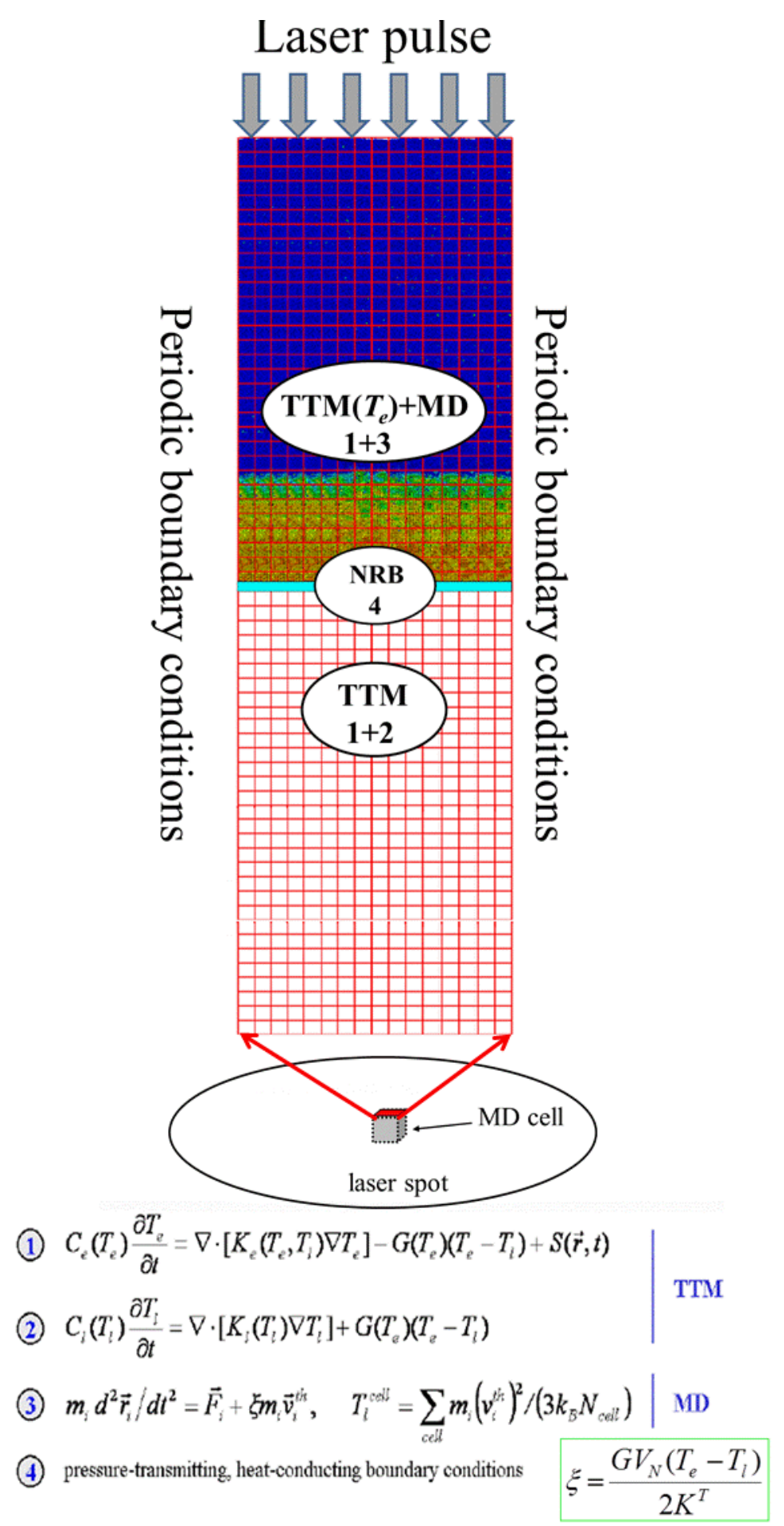

Figure 2.2: Schematics of the TTM-MD model for simulation of laser interaction with a metal target. The laser irradiation is directed from the top of the figure. 


\subsection{The TTM parameters: $C_{e}, G, K_{e}, S(z, t)$ and $C_{l}$}

To solve the TTM equation for electronic temperature $T_{e}$, four parameters, $C_{e}, G, K_{e}$, and $S(z, t)$, need to be considered as discussed below.

$C_{e}, G$ : Conventionally, the linear temperature dependence of the electron heat capacity, $C_{e}=\gamma T_{e}$, and a constant electron-phonon coupling $G$ are used in simulations of laser interaction with metals [34]. However, computational analysis based on first-principle electronic structure calculations of the electron density of states [45] predicts large deviations (up to an order of magnitude) from the common convention. It is found that these thermophysical properties are very sensitive to details of the electronic structure of the material. The strength of electronphonon coupling can either increase ( $\mathrm{Al}, \mathrm{Au}, \mathrm{Ag}, \mathrm{Cu}$ and $\mathrm{W})$, decrease (Ni and $\mathrm{Pt}$ ), or exhibit nonmonotonic changes (Ti) with increasing electron temperature. The electron heat capacity can exhibit either positive ( $\mathrm{Au}, \mathrm{Ag}, \mathrm{Cu}$ and $\mathrm{W}$ ) or negative $(\mathrm{Ni}$ and $\mathrm{Pt}$ ) deviations from the linear temperature dependence. The electron temperature dependence of electron heat capacity $C_{e}$ and electron-phonon coupling $G$ has been tabulated and can be found in [54].

$K_{e}$ : The temperature dependence of electron thermal conductivity is approximated by the free electron Drude model relationship [48], $K_{e}=\frac{1}{3} v^{2} C_{e} \tau_{e}$, where $v^{2}$ is the mean square velocity of the electrons contributing to the electron thermal conductivity, approximated by the Fermi velocity square, $v_{F}{ }^{2}$, and $\tau_{e}$ is the total electron scattering time. To determine the electron scattering time $\tau_{e}$, two scattering processes need to be considered: eletron-electron scattering and electron-phonon scattering. The temperature dependence of electron-electron scattering time $\tau_{e-e}$ and the electron-phonon scattering time $\tau_{e-p h}$ can be approximated by the following simple expressions: $\tau_{e-e}=\frac{1}{A T_{e}^{2}} \& \tau_{e-p h}=\frac{1}{B T_{l}}$, where $A$ and $B$ are constants [55]. The two scattering processes contribute together to the total scattering, i.e., the total scattering frequency $\frac{1}{\tau_{e}}$ is the sum of the electron-electron scattering frequency $\frac{1}{\tau_{e-e}}$ and the electron-phonon scattering 
frequency $\frac{1}{\tau_{e-p h}}, \frac{1}{\tau_{e}}=\frac{1}{\tau_{e-e}}+\frac{1}{\tau_{e-p h}}=A T_{e}{ }^{2}+B T_{l} . \quad$ Thus, the thermal conductivity $K_{e}=\frac{1}{3} v_{F}{ }^{2} \frac{C_{e}\left(T_{e}\right)}{A T_{e}^{2}+B T_{l}}$, where the more realistic temperature dependence of electron heat capacity $C_{e}\left(T_{e}\right)$ is taken from [45,54] as just discussed. The value of constant $A$ can be estimated within the free electron model as suggested in [56], whereas the value of constant $B$ can be obtained from the experimental value of the solid material at the melting (or room) temperature [57].

$S(z, t) \quad: \quad$ The laser source term $S(z, t)$ takes the form $S(z, t)=\frac{I_{0}}{L_{p}+L_{b}} \exp \left(-\frac{z}{L_{p}+L_{b}}\right) \exp \left(-\left(t-t_{0}\right)^{2} / 2 \sigma^{2}\right)$. No $x / y$ dependence in the laser source term indicates that the laser is uniform along lateral dimensions of the computational cell. The energy distribution in $\mathrm{z}$ direction reproduces the exponential attenuation of laser intensity with depth under the surface (Beer-Lambert law). In order to account for the ballistic energy transport occurring before the thermalization of the excited electrons, the effective energy penetration depth is approximated by the sum of the optical penetration depth and the ballistic penetration depth, $L_{p}+L_{b}$.

The effect of atomic density variations in the MD part of the model on the parameters of TTM equations $\left(C_{e}, K_{e}, G\right.$ and $\left.S(z, t)\right)$ is taken into account in solving the TTM equation of electronic temperature $T_{e}$.

To solve the TTM equation for lattice temperature $T_{l}$ in pure TTM region, the volumeteric lattice heat capacity $C_{l}$ needs to be specified. In the classical limit, $C_{l}$ is a constant that depends on the atomic density: $C_{l}=3 N k_{B}$, where $\mathrm{N}$ is the number of atoms per unit volume.

\subsection{The interatomic potential for the metals}

The ability of MD simulations to reproduce the behavior and properties of real materials is largely defined by the choice of the appropriate potential function, which describes the way how the atoms interact. Simple pair potential (e.g., Lennard-Jones potential) considers only interaction between atomic pairs according to their relative positions and is often used in 
simulations when the objective is to model a general effect. But pair potentials fall short in predicting the structures and properties of metals. For example, the vacancy formation energy is significantly overestimated by pair potentials, and the ratio between the cohesive energy and the melting temperature is underestimated by as much as 2-3 times. To overcome this limitation, Daw and Baskes proposed a novel approach, the so called embedding atom method (EAM), that enables a much better representation of the interatomic interactions in metals in 1984 [58]. Ever since then, many variants of EAM potential have been developed for different metal systems and this potential has become a standard potential for metal systems. Basically, EAM defines the total energy of the system as a sum of the total energy of pair interactions and an additional embedding energy that depends on the local electron density as described by the following equations:

$$
\begin{gathered}
E_{t o t}=\sum_{i=1}^{N} E_{i}, \quad E_{i}=F_{i}\left(\rho_{i}=\sum_{j \neq i} f_{j}\left(r_{i j}\right)\right)+\frac{1}{2} \sum_{j \neq i} \phi_{i j}\left(r_{i j}\right) \\
\vec{F}_{i}=-\nabla_{\vec{r}_{i}} E_{t o t}=-\nabla_{\vec{r}_{i}}\left[F_{i}\left(\rho_{i}\right)+\sum_{j \neq i} F_{j}\left(\rho_{j}\right)+\sum_{j \neq i} \phi_{i j}\left(r_{i j}\right)\right] \\
=-\sum_{j \neq i}\left[\left.\left.\frac{\partial F_{i}(\rho)}{\partial \rho}\right|_{\rho=\rho_{i}} \frac{\partial f_{j}(r)}{\partial r}\right|_{r=r_{i j}}+\left.\left.\frac{\partial F_{j}(\rho)}{\partial \rho}\right|_{\rho=\rho_{j}} \frac{\partial f_{i}(r)}{\partial r}\right|_{r=r_{i j}}+\left.\frac{\partial \phi_{i j}(r)}{\partial r}\right|_{r=r_{i j}}\right] \frac{\left(\vec{r}_{i}-\vec{r}_{j}\right)}{r_{i j}}
\end{gathered}
$$

The embedding energy term accounts for the energy arising from "embedding" an atom into the local electron density $\rho$ at the position of the atom. Since each of the surrounding atoms makes a contribution to the local electron density $\rho$, the EAM potential is essentially a many-body potential.

In our TTM-MD simulations of short pulse laser interactions with $\mathrm{Ag}-\mathrm{Cu}$ layered targets and pure Ag bulk targets, we choose the EAM potential suggested by Foiles, Baskes and Daw (FBD) in 1986 [59]. The potential has been parameterized for $\mathrm{Cu}, \mathrm{Ag}, \mathrm{Au}, \mathrm{Ni}, \mathrm{Pd}, \mathrm{Pt}$ and their alloys. For pure elements, the potential functions are fitted to the equilibrium lattice constants, sublimation energies, bulk moduli, elastic constants, and vacancy formation energies at zero (or room) temperature, whereas the parameters of functions describing the alloy (e.g. Ag-Cu) crossinteraction are fitted to the heat of mixing of dilute solid solutions. Even though the experimental information used to determine the potential functions is all for zero (or room) temperature solids, the thermodynamic properties (e.g. Gibbs free energy, and the thermal 
expansion coefficient) of the solid and liquid phases at high temperatures predicted by the FBD agree surprisingly well with the experiments, especially for $\mathrm{Ag} / \mathrm{Cu}[60]$.

Our implementation of the FBD EAM potential includes a cutoff function [61] that smoothly brings the interaction energies and forces to zero at a cutoff distance of $5.5 \AA$. The equilibrium melting temperatures of pure $\mathrm{Ag}(1139 \pm 2 \mathrm{~K})$ and $\mathrm{Cu}(1288 \pm 2 \mathrm{~K})$ are calculated in liquid-crystal coexistence simulations [62,63] performed at zero pressure for a system consisting of 5760 atoms $(6 \times 6 \times 40$ unit cells), with liquid-crystal interface oriented along the (001) plane of the FCC crystal. The hydrostatic zero pressure conditions are ensured by independent control over the three diagonal components of the stress tensor. The error of $\pm 2 \mathrm{~K}$ is obtained by running series of simulations at different temperatures and monitoring the evolution of the liquid-crystal interface for $2 \mathrm{~ns}$. The temperatures in the simulations were varied around the melting temperatures listed above with a step of $1 \mathrm{~K}$. The growth or disappearance of the liquid phase is reproducible outside the $\pm 2 \mathrm{~K}$ intervals around the melting temperatures, whereas within these intervals the dominance of melting or solidification is not apparent. The values of the melting temperature are in a reasonable agreement with the results of earlier coexistence calculations performed with the same potential [64,65], where the values of $1144 \mathrm{~K}$ for $\mathrm{Ag}$ and $1279 \mathrm{~K}$ for $\mathrm{Cu}$ are obtained. The melting temperatures predicted by the FBD EAM are within $7 \%$ of the experimental values of $1235 \mathrm{~K}$ for $\mathrm{Ag}$ and $1356 \mathrm{~K}$ for $\mathrm{Cu}$ [66] and have a similar relation of the melting temperature of $\mathrm{Cu}$ being slightly higher than the one of $\mathrm{Ag}$.

Moreover, the high- temperature [64] and low-temperature [67-69] parts of the Ag-Cu phase diagram calculated with the FBD EAM potential are in a good semi-quantitative agreement with the experimental eutectic phase diagram of this system. The small positive deviation of the lattice parameter of the Ag-Cu solid solution from Vegard's law, predicted with the FBD EAM potential, is also consistent with experimental data obtained for the metastable FCC phase formed by rapid quenching [67]. We conclude, therefore, that the FBD EAM potential is appropriate for the investigation of short-pulse laser processing of the $\mathrm{Ag}-\mathrm{Cu}$ layered systems and pure Ag targets. 


\subsection{Contributions}

In this part, atomic-level computer modeling based on the combined TTM-MD model is performed for $\mathrm{Ag}-\mathrm{Cu}$ layered targets and bulk $\mathrm{Ag}$ targets, with the goal of obtaining a better understanding of the ultrafast processes induced by short-pulse irradiation. The main contributions are listed below.

1) MPI parallel code: A Fortran parallel code implementing the combined TTM-MD model is written using the Message Passing Interface (MPI) communication library. Almost all TTM-MD simulations presented in this thesis are performed with this code. The code has demonstrated a very good parallelization scalability for simulations performed for systems of up to $\sim 340$ million atoms with the use of up to 24,000 computer cores. The efficient parallelization makes it possible to run large-scale simulations, which is often necessary in order to make direct connections to experimental observations. Details of the MPI parallelization are discussed in Appendix A.

2) Runaway lattice-mismatched interface: A series of atomistic simulations of femtosecond laser irradiation of $\mathrm{Ag}$ film - $\mathrm{Cu}$ substrate system are performed. In the simulations, we observe an interesting phenomenon of generation of a runaway lattice-mismatched interface with complex three-dimensional corrugated structure as a result of femtosecond laser irradiation of this layered system. The mechanism of the generation of this new interface is revealed in a detailed structural analysis. In addition, metastable $\mathrm{BCC} \mathrm{Cu}$ phase is found to form through epitaxial growth of $\mathrm{Cu}$ on FCC Ag (001) during resolidification process, which can be directly related to the experimental observations of pseudomorphic growth of $\mathrm{BCC} \mathrm{Cu}$ during deposition on $\mathrm{Ag}(001)$ face [70,71]. The factors that facilitate the epitaxial growth of BCC $\mathrm{Cu}$ on the $\mathrm{Ag}$ (001) lattice are investigated. The results of this simulation are presented in section 3.

3) Formation of stabilized BCC $\mathrm{Cu}$ : Inspired by the observation of BCC $\mathrm{Cu}$ epitaxial growth on FCC Ag (001) presented in section 3, a series of simulations are performed for femtosecond laser irradiation on different $\mathrm{Ag}-\mathrm{Cu}$ multilayer systems to extensively study the $\mathrm{BCC} \mathrm{Cu}$ formation and its stability. It is found that the BCC $\mathrm{Cu}$ can be formed and stabilized when sandwiched between two FCC Ag (001)/BCC Cu (001) interfaces. Details are presented in section 4. 
4) Explanation of experimentally observed surface swelling: A series of small-scale and large-scale simulations of femtosecond laser irradiation of bulk Ag target are performed to explain the recent experimental observation of surface swelling [19,20]. It is found that the short pulse laser-induced photomechanical processes lead to the voids nucleation, growth and coalescence. At laser fluence just below the spallation threshold, the voids can be captured by fast resolidification. The captured voids increase the volume of the surface region, leading to an effective "swelling" of the irradiated spot. The computational prediction of the generation of porous structure of a surface region of the irradiated target provides an explanation of the recently reported experimental observations of surface swelling, or "frustrated ablation," in Al and Ag targets irradiated by 100 fs laser pulses [19,20]. The results of these simulations are presented and discussed in sections 5 and 6.

5) Microstructure of surface layer treated by short pulse laser irradiation: Large-scale simulations of femtosecond laser irradiation of a Ag target have revealed generation of a $\sim 30 \mathrm{~nm}$ surface layer of nanocrystalline structure. The generation of the nanocrystalline structure generation can be attributed to the homogeneous nucleation and growth of multiple randomly oriented crystallites under the condition of strong undercooling that is achieved during the short pulse laser irradiation. The atomistic picture of the homogeneous nucleation is explored. It is expected that the generated nanocrystalline structure can result in effective hardening of the material. The results of the simulations and the structural analysis of the laser-modified surface region are presented in section 6 . 


\section{Runaway lattice-mismatched interface in an atomistic simulation of femtosecond laser irradiation of Ag film - Cu substrate system}

\subsection{Introduction}

Short pulse laser has been widely used in metallic surface processing, which can result in the material hardening or alloying of heterogeneous samples [72]. In this section, we report the results of an atomic-level simulation of a femtosecond laser interaction with a layered $\mathrm{Ag}$ film $\mathrm{Cu}$ substrate target. Thermodynamically, the $\mathrm{Ag}-\mathrm{Cu}$ binary system is characterized by a positive heat of mixing and an eutectic phase diagram, with virtually no mutual solubility at room temperature. In rapid quenching experiments, however, the metastable FCC solid solutions are commonly produced in the whole range of the alloy compositions, e.g. [73,74]. The formation of the amorphous phase is not observed in liquid quenching experiments, but has been reported for vapor quenching (co-deposition of the two components to an amorphous substrate held at liquid nitrogen temperature) [75,76], cold rolling of $\mathrm{Ag}$ and $\mathrm{Cu}$ multilayers [77], and picosecond laser quenching [78]. The extreme heating and cooling conditions realized in short pulse laser processing experiments suggest feasibility of generation of non-equilibrium compositional profiles and metastable phases in the interfacial region of the layered $\mathrm{Ag}-\mathrm{Cu}$ target. A particular focus of this section is on an unusual observation of a "runaway" lattice-mismatched interface, when the misfit dislocations associated with the lattice-mismatched interface separate from the $\mathrm{Ag}-\mathrm{Cu}$ mixing region and shift into the $\mathrm{Cu}$ substrate as a result of the resolidification process occurring under conditions of strong undercooling. A brief description of the computational model used in the simulations is given in section 3.2. The results of the simulation and a detailed structural analysis of the laser-treated target are presented and discussed in section 3.3 and are summarized in section 3.4 .

\subsection{Computational model}

The atomic mixing and structural changes generated by short pulse laser irradiation are simulated for a system consisting of a $30 \mathrm{~nm} \mathrm{Ag}$ film deposited on a bulk $\mathrm{Cu}$ substrate. The simulation is performed with the combined TTM-MD model $[34,43]$ that couples the classical 
molecular dynamics (MD) method with a continuum-level description of the laser excitation and subsequent relaxation of the conduction band electrons, based on the two-temperature model (TTM) [49]. The model accounts for the fast electron heat conduction in the metal target and provides an adequate representation of the fast heating and cooling of the surface region of the target. The basics of the TTM-MD model have been reviewed in section 2.3 and a complete description is given in Refs. [34]. The computational setup used in the simulation is similar to the one used for the Au-Cu system in Ref. [44] as shown in Fig. 3.1. Briefly, the atomic-level MD representation is used only for the top $230 \mathrm{~nm}$ part of the target (30 nm Ag film and a top $200 \mathrm{~nm}$ part of the $\mathrm{Cu}$ substrate), whereas the heat transfer in the deeper part of the target is described by coupled electron and lattice heat conduction equations (two-temperature model (TTM) [49]) solved down to the depth of $2.4 \mu \mathrm{m}$. This depth is chosen so that no significant increase in the electron or lattice temperatures is observed at the bottom of the computational domain during the time of the simulation.

The interatomic interactions in the MD part of the system are described by the embedded atom method (EAM) potential in the form suggested by Foiles, Baskes, and Daw (FBD) [59]. The basics of the EAM potential have been reviewed in section 2.5 and as discussed there, the FBD EAM potential is appropriate for the investigation of short-pulse laser processing of the Ag$\mathrm{Cu}$ layered system. First, the potential gives a good prediction of the experimental thermodynamic properties for the pure $\mathrm{Ag}$ and $\mathrm{Cu}$ [60]. Moreover, the high- temperature [64] and low-temperature [67-69] parts of the $\mathrm{Ag}-\mathrm{Cu}$ phase diagram calculated with the FBD EAM potential are in a good semi-quantitative agreement with the experimental eutectic phase diagram of this system. 


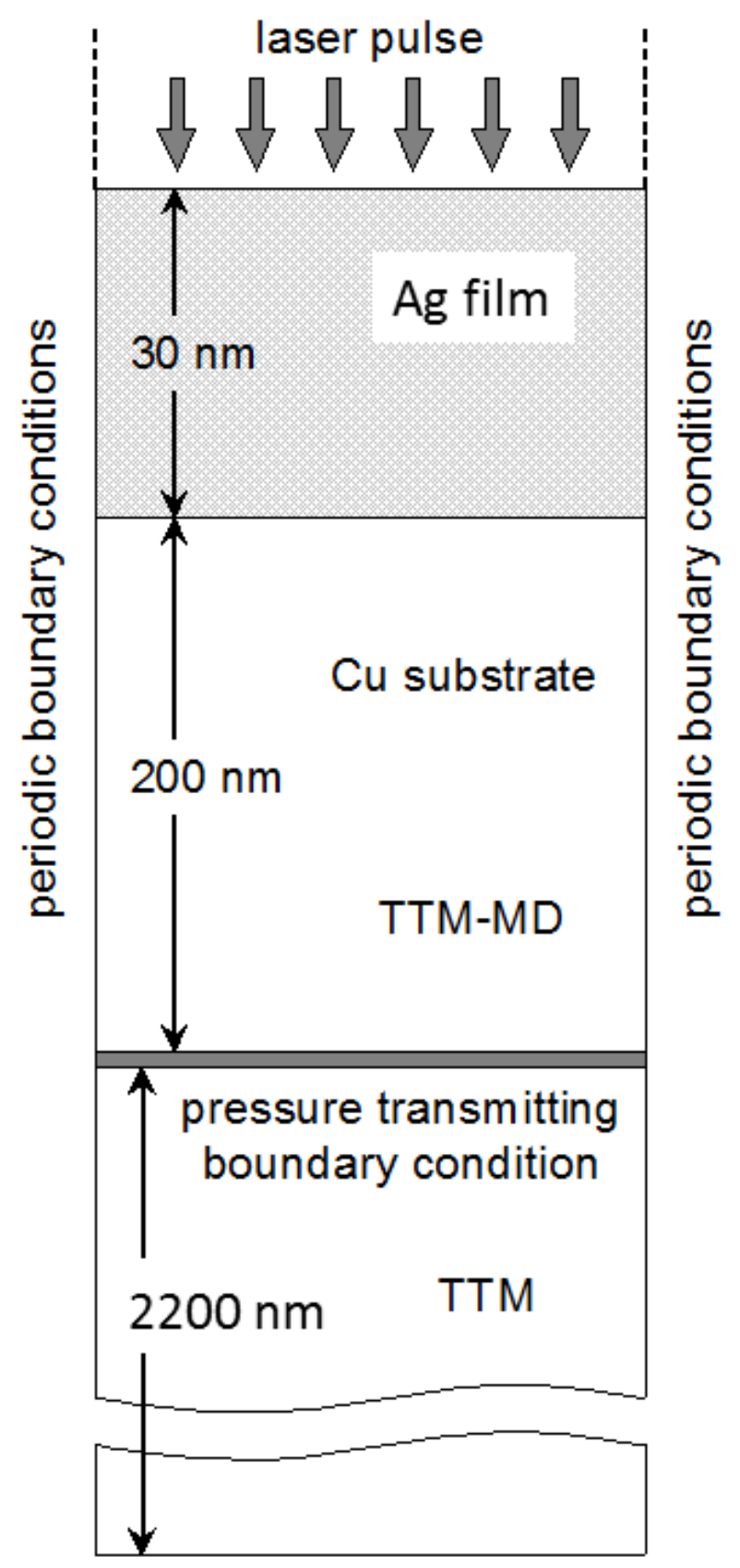

Figure 3.1: Schematic sketch of the simulation setup. The $30 \mathrm{~nm} \mathrm{Ag}$ film and a $200 \mathrm{~nm}$ part of the $\mathrm{Cu}$ substrate are represented with atomic-level resolution, using the TTM-MD model, whereas the electron heat conduction in the deeper part of the substrate is simulated with the conventional TTM. 
The top $200 \mathrm{~nm}$ part of the $\mathrm{Cu}$ substrate and the $30 \mathrm{~nm} \mathrm{Ag}$ film in the initial system are represented by FCC crystallites with lateral dimensions of $3.27 \mathrm{~nm} \times 3.27 \mathrm{~nm}$ at $300 \mathrm{~K}$ and periodic boundary conditions imposed in the directions parallel to the surface of the target. At the bottom of the MD part of the model, a special pressure-transmitting boundary condition [51,52] is applied in order to avoid an artificial reflection of the laser-induced pressure wave generated in the surface region by the fast laser energy deposition. The film and the target have the same crystallographic orientation, with (001) planes oriented parallel to the surface of the target. Although the FBD EAM potential is fitted to the room-temperature values of lattice constants and other parameters [59], the fitting is done using static calculations that do not account for the thermal expansion from zero to room temperature. As a result, the potential overpredicts the finite temperature values of the lattice parameter, e.g. $a_{C u}=3.63 \AA$ and $a_{A g}=$ $4.11 \AA$ are determined in constant zero pressure MD simulations performed at $300 \mathrm{~K}$ (compared to the experimental values $a_{C u}=3.61 \AA$ and $a_{A g}=4.09 \AA$ [48]). Nevertheless, the relative values of the lattice parameters and the corresponding misfit are similar for both experimental values and the MD results at $300 \mathrm{~K}, 9 a_{C u}=7.95 a_{A g}$ and $\left(a_{A g}-a_{C u}\right) / a_{C u}=0.13$, suggesting that the discrepancy between the calculated and experimental lattice parameters does not affect the properties of the $\mathrm{Ag}-\mathrm{Cu}$ interface.

The lateral size of the computational system corresponds to 9 FCC unit cells in each direction for the $\mathrm{Cu}$ substrate and 8 slightly compressed FCC unit cells for the Ag film at $300 \mathrm{~K}$. Before applying laser irradiation, the initial system is equilibrated in a MD simulation performed at $300 \mathrm{~K}$ for $100 \mathrm{ps}$. The equilibration results in the formation of a semicoherent interface with an array of misfit dislocations parallel to $\langle 110\rangle$ directions in the plane of the interface. The atomic structure of the interface is shown in Fig. 3.2, where the atoms are colored by their type in Fig. 3.2a and based on the output of a modified version [79] of the structural analysis suggested in Ref. [80] in Fig. 3.2b. The latter representation allows for an easy identification of the network of misfit dislocations, with atoms that belong to the dislocation cores colored yellow and green. 


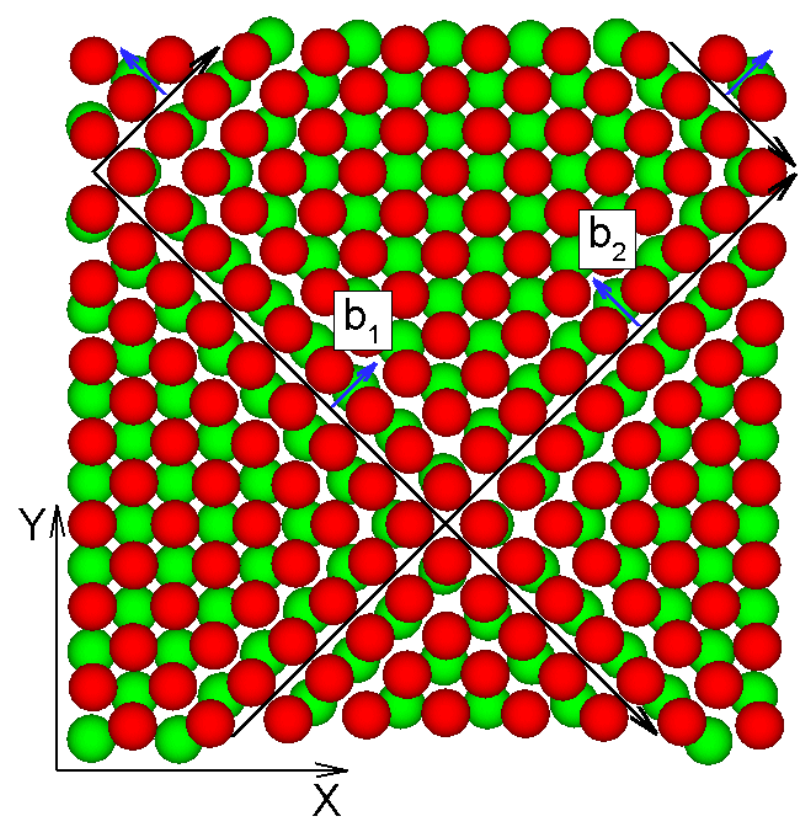

(a)

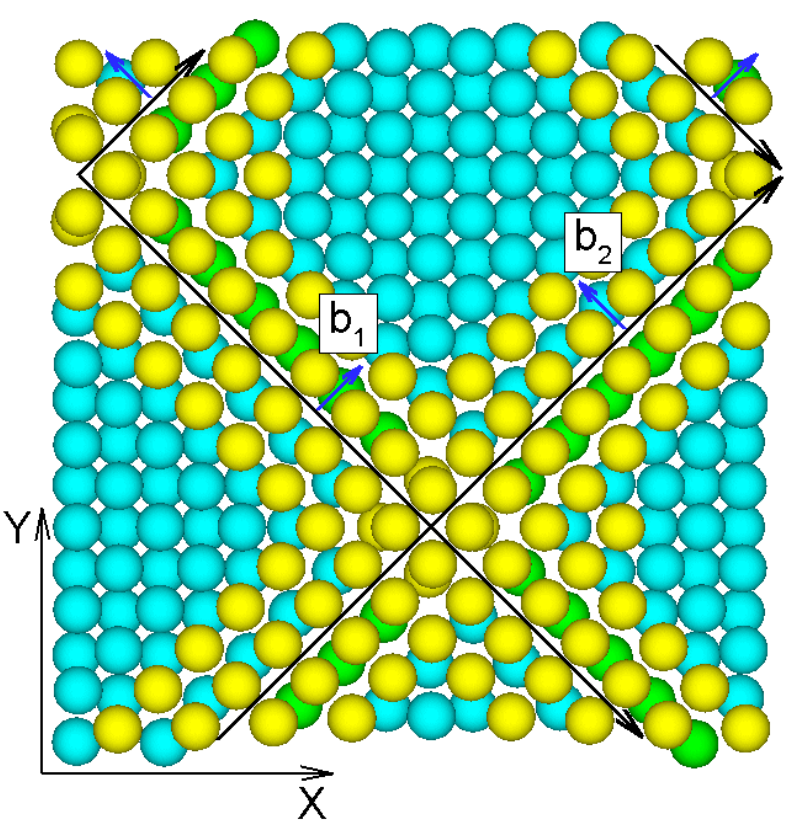

(b)

Figure 3.2: Initial configuration of the $\mathrm{Ag}-\mathrm{Cu}$ interface equilibrated at $300 \mathrm{~K}$. Two atomic layers adjacent to the interface are shown, with atoms colored by their type (red for $\mathrm{Cu}$ and green for $\mathrm{Ag}$ ) in (a) and based on the output of a modified version [79] of the structural analysis suggested in Ref. [80] (light blue and green atoms belong to atomic configurations that correspond to FCC and HCP structures, respectively, the structure around the yellow atoms cannot be assigned to any crystal structure) in (b). The lines of misfit dislocations are shown by black arrows with small blue arrows indicating the corresponding Burgers vectors, $\vec{b}_{1}=\frac{1}{2}[110]$ and $\vec{b}_{2}=\frac{1}{2}[\overline{1} 10]$.

The electron temperature dependences of the thermophysical material properties included in the TTM equation for the electron temperature (electron-phonon coupling factor and electron heat capacity) are taken in the form that account for the thermal excitation from the electron states below the Fermi level [45,54]. The electron thermal conductivity is described by the Drude model relationship, $K_{e}\left(T_{e}, T_{l}\right)=v^{2} C_{e}\left(T_{e}\right) \tau_{e}\left(T_{e}, T_{l}\right) / 3$, where $C_{e}\left(T_{e}\right)$ is the electron heat capacity, $v^{2}$ is the mean square velocity of the electrons contributing to the electron heat conductivity, approximated in this work as the Fermi velocity squared, $v_{\mathrm{F}}^{2}$, and $\tau_{e}\left(T_{e}, T_{l}\right)$ is the total electron scattering time defined by the electron-electron and electron-phonon scattering 
rates, $1 / \tau_{e}=1 / \tau_{e-e}+1 / \tau_{e-p h}=A T_{e}^{2}+B T_{l}$. The values of the coefficient $A\left(2.66 \times 10^{6} \mathrm{~s}^{-1} \mathrm{~K}^{-2}\right.$ for $\mathrm{Cu}$ and $3.57 \times 10^{6} \mathrm{~s}^{-1} \mathrm{~K}^{-2}$ for $\mathrm{Ag}$ ) are estimated within the free electron model, following the approach suggested in [56]. The values of the coefficient $B\left(2.41 \times 10^{11} \mathrm{~s}^{-1} \mathrm{~K}^{-1}\right.$ for $\mathrm{Cu}$ and $1.12 \times 10^{11} \mathrm{~s}^{-1} \mathrm{~K}^{-1}$ for $\mathrm{Ag}$ ) are obtained from the experimental values of the thermal conductivity of solid $\mathrm{Cu}$ and $\mathrm{Ag}$ at the melting temperature, $330 \mathrm{Wm}^{-1} \mathrm{~K}^{-1}$ for $\mathrm{Cu}$ and $363 \mathrm{Wm}^{-1} \mathrm{~K}^{-1}$ for $\mathrm{Ag}$ [57]. In the region adjacent to the $\mathrm{Ag}-\mathrm{Cu}$ interface, the material properties included in the TTM equation for the electron temperature are calculated as linear combinations of the properties of individual components taken with weights equal to the local atomic concentrations.

The irradiation of the layered target with a 200 fs laser pulse is represented through a source term added to the equation for the electron temperature. The source term simulates excitation of the conduction band electrons by a laser pulse with a Gaussian temporal profile and reproduces the exponential attenuation of laser intensity with depth under the surface (BeerLambert law). In order to account for the ballistic energy transport occurring before the thermalization of the excited electrons [44,46,81], the optical absorption depth, $L_{p}$, is substituted in the source term by an effective range of the laser energy deposition, $L_{p}+L_{b}[34,44,46]$. The strong dependence of the electron-electron scattering on the excitation energy [81] and modification of the scattering rates in the $\mathrm{Ag}-\mathrm{Cu}$ interfacial region introduce uncertainty to the estimation of the effective depth of the ballistic energy transport [44]. In the simulation here, we assume the effective range of the laser energy deposition to be $L_{p}+L_{b}=100 \mathrm{~nm}$. As shown in Ref. [44] for the Au-Cu system, the variation of $L_{p}+L_{b}$ affects the values of laser fluence required for the onset of the interfacial melting or complete melting of the film, but do not change the qualitative picture of the laser-induced processes.

\subsection{Results and discussion}

\subsubsection{Laser heating and sub-surface melting}

The simulation is performed for the layered $\mathrm{Ag}-\mathrm{Cu}$ system irradiated by a $200 \mathrm{fs}$ laser pulse at an absorbed fluence of $0.13 \mathrm{~J} / \mathrm{cm}^{2}$. This fluence is $\sim 1.3$ times higher than the fluence required for the onset of sub-surface melting of the interfacial region $\left(\sim 0.10 \mathrm{~J} / \mathrm{cm}^{2}\right)$ and $\sim 1.2$ times lower than the threshold fluence for photomechanical spallation [82] of the Ag film from the $\mathrm{Cu}$ substrate $\left(\sim 0.16 \mathrm{~J} / \mathrm{cm}^{2}\right)$. The contour plots in Fig. 3.3 show the evolution of the lattice 
temperature, pressure and density in the irradiated target. Although immediately after the laser pulse the electron temperature in the $\mathrm{Ag}$ film is substantially higher than the one in the underlying $\mathrm{Cu}$ substrate, the lattice temperature increases faster at the $\mathrm{Cu}$ side of the $\mathrm{Ag}-\mathrm{Cu}$ interface, Fig. 3.3a. This preferential sub-surface heating can be explained by substantially stronger electron-phonon coupling in $\mathrm{Cu}$ compared to $\mathrm{Ag}$ (room temperature values of the electron-phonon coupling factor are $\sim 5.5 \times 10^{16} \mathrm{Wm}^{-3} \mathrm{~K}^{-1}$ for $\mathrm{Cu}$ and $\sim 2.5 \times 10^{16} \mathrm{Wm}^{-3} \mathrm{~K}^{-1}$ for $\mathrm{Ag}$ and the difference becomes larger with increasing electron temperature due to the stronger contribution of thermally excited $d$-band electrons in the case of $\mathrm{Cu}$ [45,54]). A similar effect of sub-surface heating in femtosecond laser interactions with layered targets has been predicted in TTM calculations performed for Au-Cr targets [83] and in TTM-MD simulations of Au-Cu targets [44]. In both cases, the faster increase of the lattice temperature in the $\mathrm{Cr}$ or $\mathrm{Cu}$ layers was attributed to the stronger electron-phonon coupling in $\mathrm{Cr}$ and $\mathrm{Cu}$ compared to $\mathrm{Au}$.

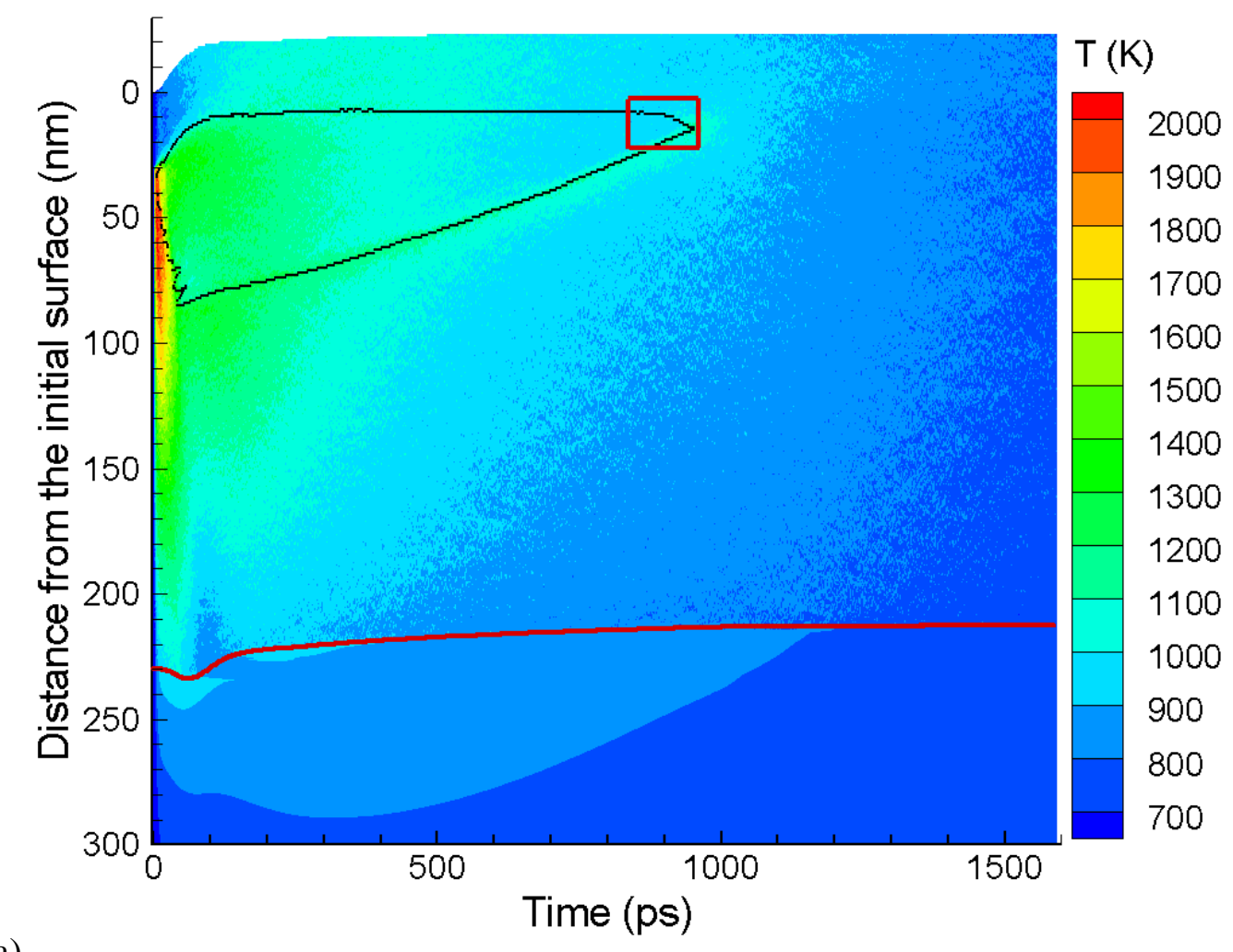

(a) 
(b)
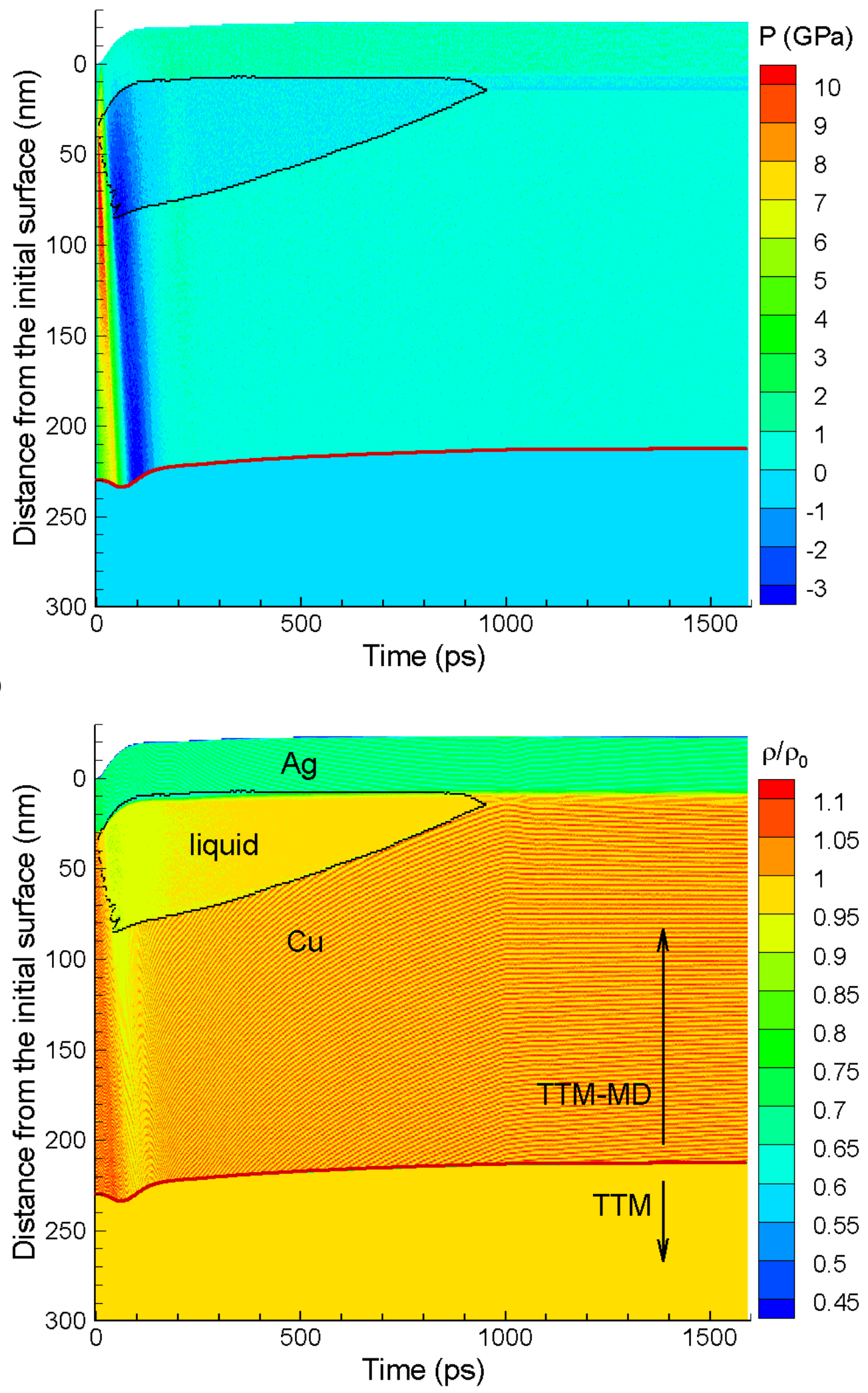

(c) 
Figure 3.3: Contour plots of the lattice temperature (a), pressure (b), and density (c) for a simulation of a $\mathrm{Ag}-\mathrm{Cu}$ target irradiated with a $200 \mathrm{fs}$ laser pulse at an absorbed fluence of 0.13 $\mathrm{J} / \mathrm{cm}^{2}$. The laser pulse is directed along the Y-axis, from the top of the contour plots. The black lines separate the melted regions from the crystalline parts of the target. The red lines separate the atomistic (TTM-MD) and continuum (TTM) parts of the computational domain. The red rectangle in (a) shows the region and time interval for which snapshots are shown in Fig. 3.4. The density scale in (c) is normalized to the density of substrate $\mathrm{Cu}$ before the irradiation (at 300 $\mathrm{K}), \rho_{0}$. The fine "fingerprint"-type pattern observed in the crystalline parts of the target in the density plot is an artifact of the data analysis method - the physical properties are averaged over $1 \mathrm{~nm}$ thick slices and the stepwise variation of the number of atoms (and density) in each slice corresponds to an atomic layer entering/leaving the slice due to the material displacement in response to the pressure waves and thermal expansion.

The rapid heating of the upper part of the $\mathrm{Cu}$ substrate leads to the build up of high compressive stresses that drive compressive pressure waves deeper into the bulk of the target and in the direction of the free surface, Fig. 3.3b. The reflection of the wave from the free surface of the Ag film transforms it to an unloading tensile wave that follows the compressive wave propagating into the bulk of the $\mathrm{Cu}$ substrate. Both compressive and tensile components of the laser-generated pressure wave are transmitted through the pressure-transmitting boundary at the bottom of the atomistic part of the computational model without any noticeable reflection.

The rising lattice temperature in the top part of the substrate quickly exceeds the melting temperature of $\mathrm{Cu}$ and leads to the fast homogeneous melting of $\sim 55 \mathrm{~nm}$ region of the substrate. The melted part of the target is outlined by the black lines in Fig. 3.3 and is particularly apparent in the density plot (Fig. 3.3c), where the difference in densities of liquid $\mathrm{Cu}$, solid $\mathrm{Cu}$, and solid Ag makes it easy to distinguish the melted region from the rest of the substrate. Similarly, the boundary of the melted region can be identified by a jump in the level of pressure in Fig. 3.3b. The hydrostatic pressure conditions and complete relaxation of stresses are quickly achieved in the melted part of the substrate, whereas the laser-induced compressive stresses in the crystalline part of the target cannot completely relax by the uniaxial expansion in the direction normal to the surface. The uniaxial expansion of the crystalline part of the target results in anisotropic lattice 
deformations and corresponding quasi-static anisotropic stresses that slowly decrease with cooling of the surface region by heat conduction to the bulk of the target.

The melting starts just below the $\mathrm{Ag}-\mathrm{Cu}$ interface at $\sim 10 \mathrm{ps}$ and continues during the following $40 \mathrm{ps}$, reaching the maximum depth of $\sim 85 \mathrm{~nm}$ below the initial position of the surface of the target. The nucleation of the liquid regions at the end of the melting process is assisted by the tensile stresses associated with the unloading wave propagating through the melting region. A relatively small ( $\sim 3 \mathrm{~nm}$ deep) part of the $\mathrm{Ag}$ film adjacent to the $\mathrm{Ag}-\mathrm{Cu}$ interface also undergoes melting.

\subsubsection{Cooling and resolidification}

The strong temperature gradient and the fast electronic heat conduction in $\mathrm{Cu}$ lead to the rapid cooling of the surface region of the irradiated target and, by the time of $100 \mathrm{ps,} \mathrm{the}$ temperature in the whole melted region drops below the equilibrium melting temperature of $\mathrm{Cu}$. The velocity of the resolidification front increases with increasing undercooling of the melted $\mathrm{Cu}$ and reaches the maximum velocity of $\sim 100 \mathrm{~m} / \mathrm{s}$ by the time of $\sim 700 \mathrm{ps,} \mathrm{Fig.} \mathrm{3.3.}$

From the Ag side of the melted region, a noticeable advancement of the resolidification front is observed only starting from $\sim 400 \mathrm{ps}$, when the temperature of the Ag-Cu interface drops down to $\sim 1080 \mathrm{~K}$, Fig. 3.4. This second resolidification front propagates with a relatively low average velocity of $\sim 8 \mathrm{~m} / \mathrm{s}$ for $\sim 275 \mathrm{ps}$ (until the time of $\sim 675 \mathrm{ps}$ ) and then slows down and remains almost immobile for the following 165 ps. The low velocity of the second resolidification front can be explained by slower solidification kinetics in binary systems and, more importantly, by the depression of the melting temperature (liquidus line) with respect to the pure $\mathrm{Ag}$ and $\mathrm{Cu}$. The range of diffusional mixing of $\mathrm{Cu}$ and $\mathrm{Ag}$ atoms quickly extends up to the solid part of the Ag film and the resolidification front propagates through the melt with increasing concentration of $\mathrm{Cu}$ atoms. The propagation of the resolidification comes to a temporal halt at $\sim 675 \mathrm{ps}$, when the composition of the melt becomes close to the eutectic composition of $\sim 36$ at.\% Cu estimated for the FBD EAM Ag-Cu system [64]. 


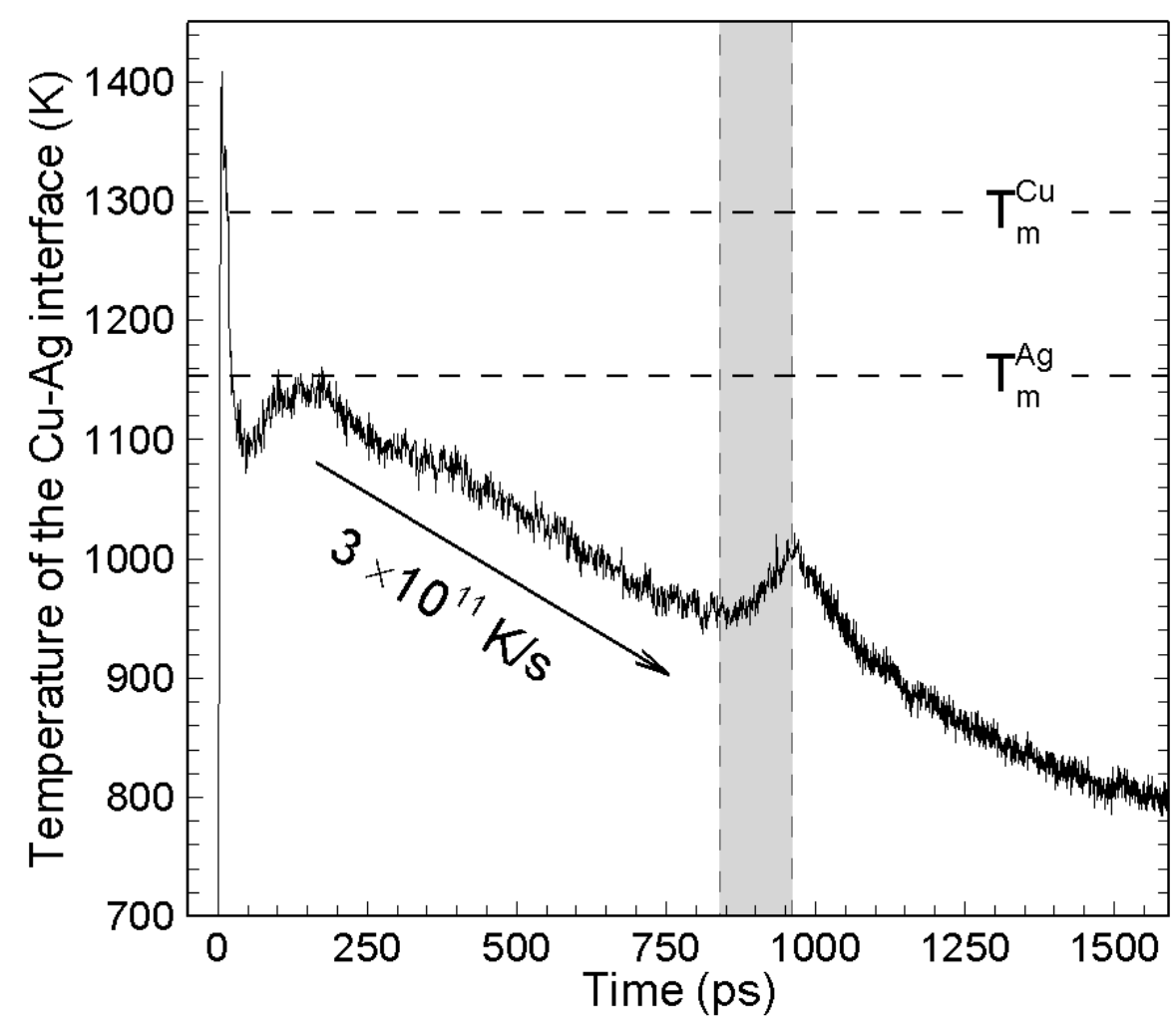

Figure 3.4: Time dependence of the lattice temperature of the interfacial region of a $\mathrm{Ag}-\mathrm{Cu}$ target irradiated with a $200 \mathrm{fs}$ laser pulse at an absorbed fluence of $0.13 \mathrm{~J} / \mathrm{cm}^{2}$. The horizontal dashed lines show the equilibrium melting temperatures of the FBD EAM Cu and Ag materials. The lattice temperature is calculated from the average kinetic energy of atoms in a $5 \mathrm{~nm}$ layer adjacent to the interface. The gray area marks the time interval from 840 to $960 \mathrm{ps,} \mathrm{during}$ which a resolidification front propagates through the region of atomic mixing from the side of the $\mathrm{Ag}$ film and meets another resolidification front that propagates from the side of the $\mathrm{Cu}$ substrate (see Fig. 3.5). The release of the latent heat of melting upon solidification of the interfacial region is responsible for the transient temperature increase during the time of the solidification.

While the resolidification front from the Ag side remains stuck in the mixing region and the resolidification front from the $\mathrm{Cu}$ side propagates at its maximum velocity, the temperature of the $\mathrm{Ag}-\mathrm{Cu}$ interface continue to decrease with the cooling rate of $\sim 3 \times 10^{11} \mathrm{~K} / \mathrm{s}$, Fig. 3.4. Finally, when at $\sim 840 \mathrm{ps}$ the temperature of the interface drops down to $\sim 950 \mathrm{~K}$ (close to the estimated eutectic temperature of $904 \mathrm{~K}$ [64]), the resolidification front on the Ag side starts to move again and slowly, with an average velocity of $\sim 25 \mathrm{~m} / \mathrm{s}$, passes through the remaining part of the mixing region within the following $60 \mathrm{ps}$. After crossing the mixing region, the 
resolidification front sharply accelerates and propagates through the undercooled $\mathrm{Cu}$ with a velocity similar to the one of the resolidification front propagating from the $\mathrm{Cu}$ substrate, $\sim 100$ $\mathrm{m} / \mathrm{s}$. The atomic-level view of the resolidification process in the vicinity of the mixing region is provided by snapshots of atomic configurations shown in Fig. 3.5, where the positions of the two resolidification fronts are marked by white vertical lines. The two resolidification fronts meet each other at $\sim 955 \mathrm{ps}$ and form a new lattice-mismatched interface at a distance of $\sim 6.5 \mathrm{~nm}$ from the center of the mixing region. This new mismatched interface is remarkable since it is located inside the $\mathrm{Cu}$ substrate and is separating the bulk of the FCC Cu substrate from an intermediate $\mathrm{Cu}$ layer with a structure that forms as a result of the pseudomorphic epitaxial growth on (001) Ag. The structure of the new mismatched interface, the intermediate $\mathrm{Cu}$ layer, and the factors that facilitate the epitaxial growth of $\mathrm{Cu}$ on the $\mathrm{Ag}$ lattice are discussed below. 

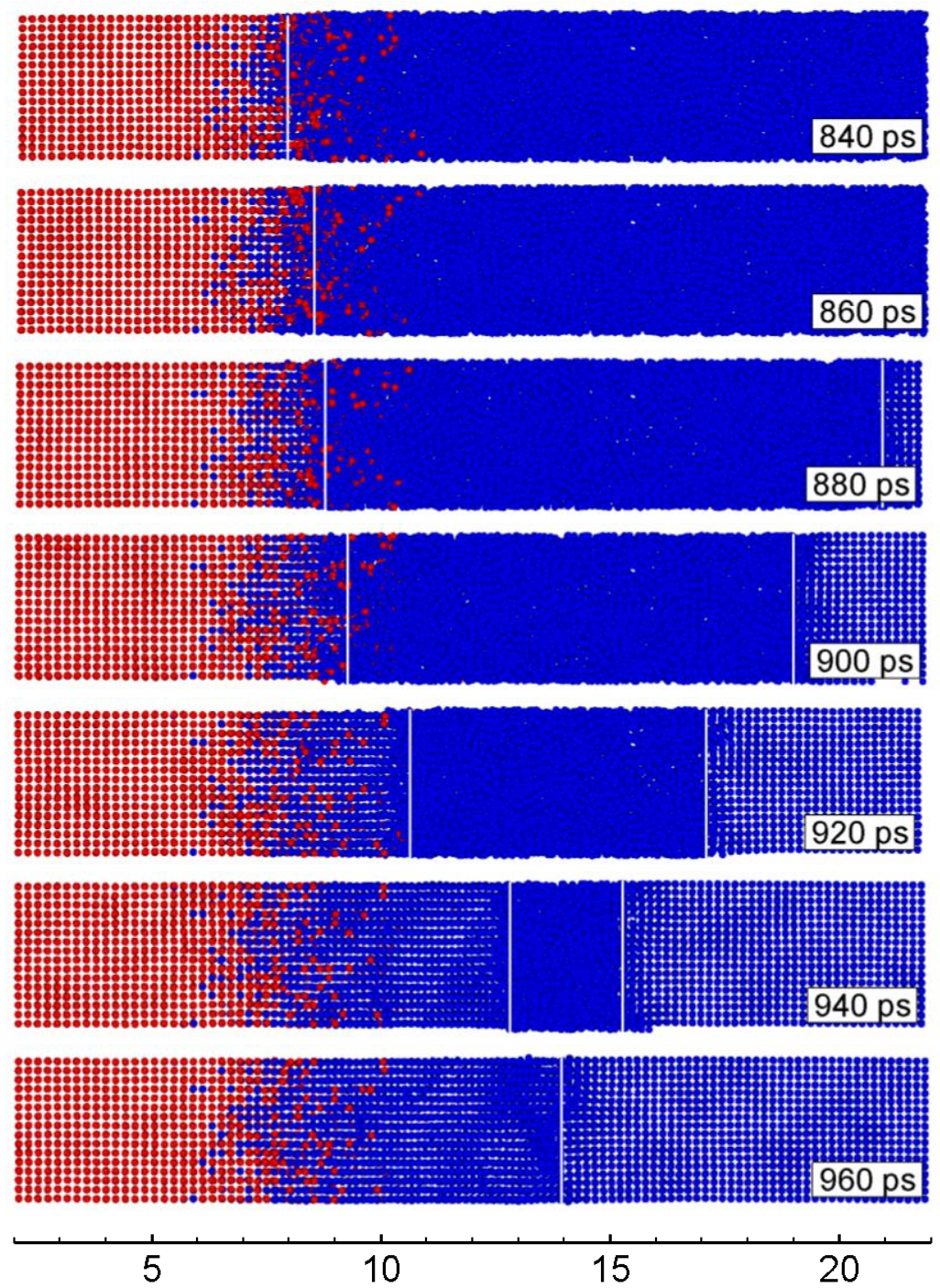

Distance from the initial surface $(\mathrm{nm})$

Figure 3.5: Snapshots of the evolution of the atomic structure in the region of $\mathrm{Ag}-\mathrm{Cu}$ interface in the simulation illustrated by the contour plots in Fig. 3.3. The snapshots are taken for the region and the time interval marked in Fig. 3.3a by the red rectangle. Atoms are colored according to their type - $\mathrm{Ag}$ atoms are colored red and $\mathrm{Cu}$ atoms are colored blue. To reduce the thermal noise in atomic positions, the configurations are quenched for $0.2 \mathrm{ps}$ using a velocity dampening technique (see text). The fast quenching does not introduces any structural changes to the atomic configurations but makes the visual analysis more straightforward. The vertical white lines in each snapshot show the approximate locations of resolidification fronts propagating from the $\mathrm{Ag}$ (left) and $\mathrm{Cu}$ (right) sides of the system and meeting each other at $\sim 955$ ps. 


\subsubsection{Structure of the resolidified region}

To better understand the unusual phenomenon of the "runaway" lattice-mismatched interface, when the lattice-mismatched interface separates from the $\mathrm{Ag}-\mathrm{Cu}$ mixing region and shifts into the $\mathrm{Cu}$ substrate, we consider the composition profiles and distributions of structural characteristics in a part of the target that includes both the atomic mixing region and the new lattice-mismatched interface, Fig. 3.6. The analysis is performed for an atomic configuration obtained by the end of the simulation, $1.59 \mathrm{~ns}$ after the laser pulse. The average composition and structural characteristics are calculated for individual atomic planes parallel to the surface of the layered system. These atomic planes correspond to (002) planes in the original FCC structures of the $\mathrm{Cu}$ substrate and $\mathrm{Ag}$ film. To reduce the thermal noise in atomic positions, the analysis is preceded by quenching of the atomic configuration for 0.2 ps using a velocity dampening technique, where the velocity of each individual atom is set to zero at the time when the kinetic energy of the atom maximizes. The fast quenching does not introduce any structural changes to the atomic configuration but makes the visualization and structural analysis more straightforward.

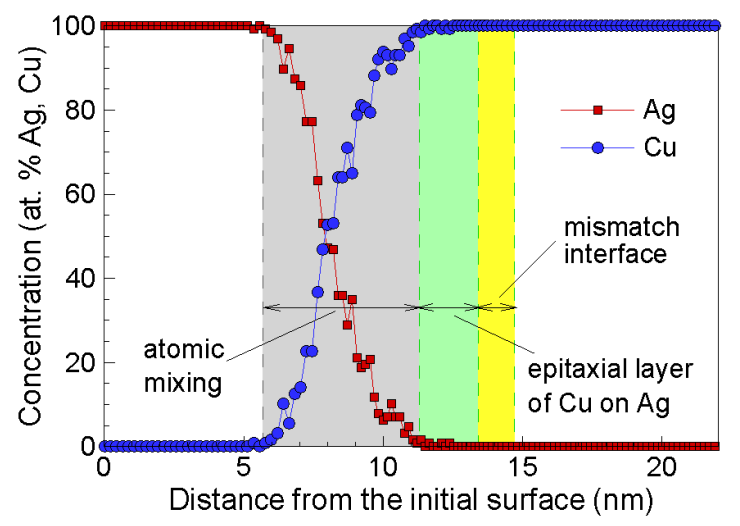

(a)

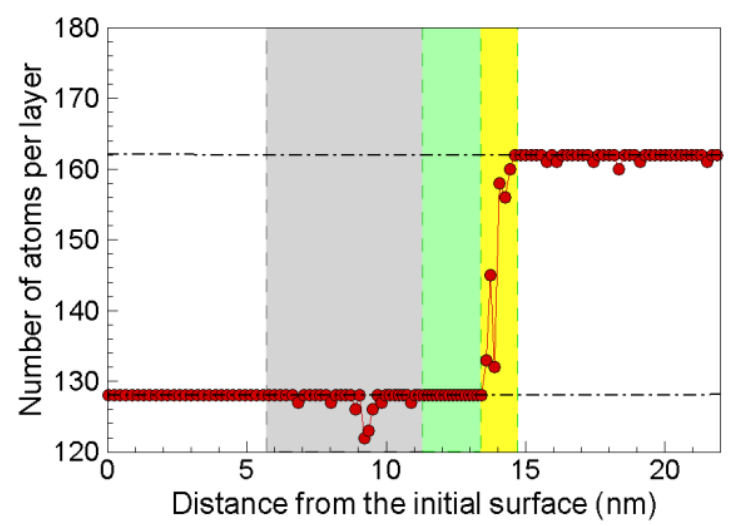

(b) 
(c)

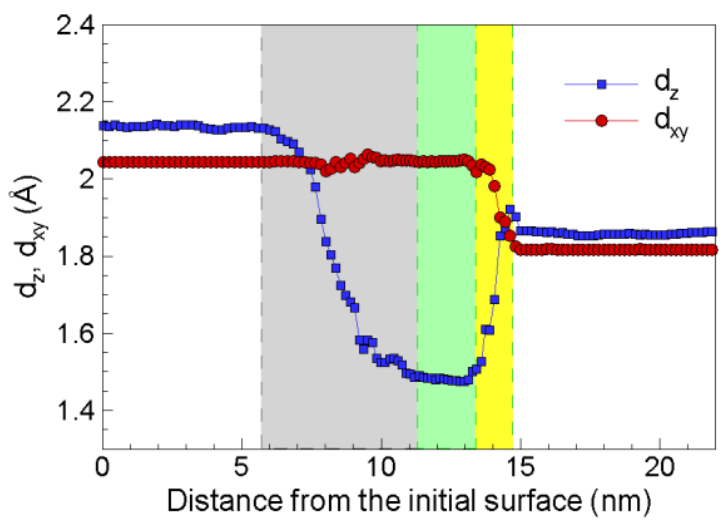

(d)

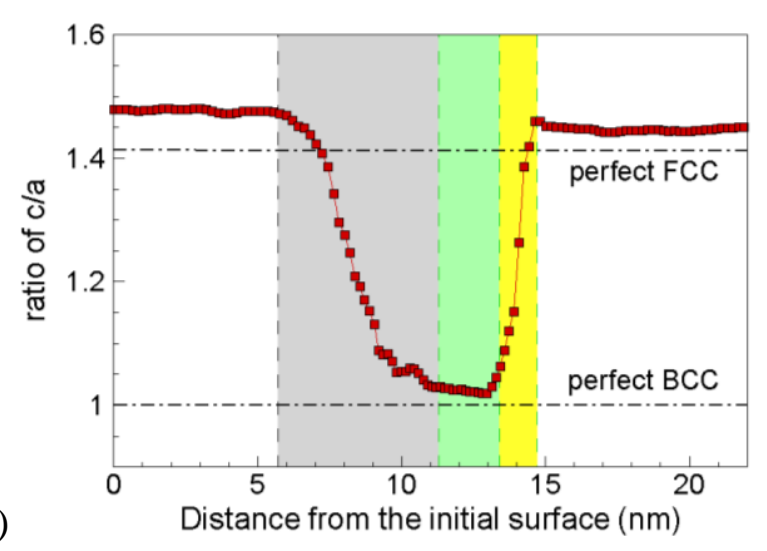

(e)

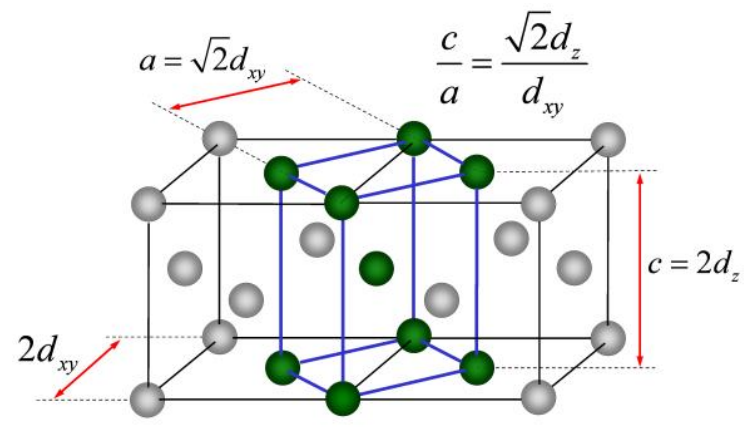

Figure 3.6: Concentration profiles (a), number of atoms in atomic planes (b), spacing between $\{200\}$ planes of the original FCC structure (c), and ratio c/a of the BCT lattice constants (d) shown for a region adjacent to the $\mathrm{Ag}-\mathrm{Cu}$ interface. The analysis is performed for an atomic configuration obtained by the end of the simulation (1.59 ns after the laser pulse) and quenched by applying the velocity dampening technique for 0.2 ps (the snapshots of the corresponding atomic configuration are shown in Fig. 3.7(a-c). The definitions of the structural parameters shown in the plots are illustrated in (e). Each point in the plots is calculated for an individual atomic plane parallel to the surface of the layered system. The atomic planes correspond to (002) planes in the original FCC structures of the $\mathrm{Cu}$ substrate and the Ag film. In (c), $\mathrm{d}_{\mathrm{xy}}$ corresponds 
to the average spacing between the neighboring atomic planes that correspond to (200) and (020) planes in the FCC structure, $d_{z}$ corresponds to the average spacing between the neighboring (002) planes of the FCC structure, and atoms that do not belong to FCC or BCC/BCT atomic configurations are excluded from the averaging. In (d), the ratio c/a is defined as shown in (e), so that the perfect FCC and BCC structures are treated as general BCT structures with ideal ratio of $\mathrm{c} / \mathrm{a}=\sqrt{2}$ and 1 , respectively. The gray, green and yellow areas in the plots mark the regions of atomic mixing, pseudomorphic epitaxial layer of $\mathrm{Cu}$ on $\mathrm{Ag}$, and corrugated lattice-mismatched interface consisting of stacking fault pyramids illustrated in Fig. 3.8, respectively.

The concentration profiles produced by the laser processing of the layered target are shown in Fig. 3.6a. The width of the region of atomic mixing, shown as a gray area in Fig. 3.6, is $\sim 5.6 \mathrm{~nm}$. This width is substantially wider compared to the width of the composition profiles predicted in Monte Carlo simulations for equilibrium interfaces, $\sim 1 \mathrm{~nm}$ [69]. The difference can be explained by the high rates of the cooling and resolidification processes realized in short pulse laser processing. The high atomic mobility and unlimited solubility in the liquid state result in a rapid mixing in the transiently melted interfacial region. The subsequent fast resolidification happens within hundreds of picoseconds and does not allow for any significant compositional changes in the mixing region, thus resulting in the formation of non-equilibrium concentration profiles. The concentration profiles in Fig. 3.6a exhibit a noticeable asymmetry with respect to the location of the equal concentrations of $\mathrm{Cu}$ and $\mathrm{Ag}$. The location where the concentration of the components is 50 at.\% is shifted by $\sim 0.6 \mathrm{~nm}$ with respect to the center of the mixing region. The asymmetry in the concentration profiles can be attributed to the asymmetry in the interfacial melting that takes place mainly in the $\mathrm{Cu}$ substrate and only affects a shallow (up to $3 \mathrm{~nm}$ ) part of the Ag film. The liquid-crystal interface provides a natural limit for the diffusion of $\mathrm{Cu}$ atoms into the Ag film and the advancement of the resolidification front from the Ag side confines the atomic mixing to an increasingly smaller part of the film. For example, one can see from the top snapshot in Fig. 3.5 that the Ag side of the interface has resolidified by the time of $840 \mathrm{ps,}$ whereas the $\mathrm{Cu}$ side is still melted and is a subject of continued diffusion of $\mathrm{Ag}$ atoms into the $\mathrm{Cu}$ substrate.

The shift of the lattice-mismatched interface from the region of atomic mixing to the $\mathrm{Cu}$ substrate is apparent by comparing the concentration profiles in Fig. 3.6a with the distribution of 
the number of atoms per atomic monolayer shown in Fig. 3.6b. Even though there are almost no $\mathrm{Ag}$ atoms below the depth of $\sim 11 \mathrm{~nm}$, the region where each layer consists of $\sim 128$ atoms, as in the Ag crystal, extends down to $\sim 13.4 \mathrm{~nm}$. The number of atoms per atomic layer jumps from 128 to 162 , the value characteristic of the $\mathrm{Cu}$ FCC crystal, within a narrow region from 13.4 to $14.7 \mathrm{~nm}$ (marked by yellow color in Fig. 3.6), signifying the new location of the latticemismatched interface. A detailed discussion of the atomic structure of the mismatched interface is provided in section 3.3.4.

Now we consider the structure of the intermediate $2 \mathrm{~nm}$ wide region that separates the regions of $\mathrm{Ag}-\mathrm{Cu}$ mixing and the new lattice-mismatched interface. As discussed in section 3.3.2, the structure of this region, marked by green color in Fig. 3.6, is formed as a result of coherent epitaxial growth of $\mathrm{Cu}$ on $\mathrm{Ag}$, with $\mathrm{Cu}$ atoms adopting the packing density of (002) planes of the Ag FCC lattice. Similarly to the number of atoms per atomic plane (Fig. 3.6b), the average spacing $d_{x y}$ between the neighboring (200) or (020) planes, defined as shown in Fig. 3.6e, does not experience any noticeable change upon the transition from the Ag to the $\mathrm{Cu}$ part of the system and drops to the value characteristic of the $\mathrm{Cu}$ crystal only at the lattice-mismatched interface, Fig. 3.6c. On the contrary, the spacing $d_{z}$ between the (002) atomic planes gradually decreases with decreasing concentration of $\mathrm{Ag}$, saturates at an almost constant value of $\sim 1.48 \AA$ in the region of the epitaxial layer of $\mathrm{Cu}$ on $\mathrm{Ag}$, and increases to the value of the $\mathrm{Cu}$ substrate upon crossing the mismatched interface. The large disparity between the values of $d_{z}$ and $d_{x y}$ in the epitaxial layer suggests that the structure changes upon the transition through the mixing region.

In the regions away from the $\mathrm{Ag}-\mathrm{Cu}$ intermixing and epitaxial layers, the difference in the levels of $d_{z}$ and $d_{x y}$ reflects the lateral confinement of the FCC lattice in the directions parallel to the surface of the irradiated target. The surface region of the target heated by the laser pulse can only expand in the direction normal to the surface, leading to the split between $d_{z}$ that increases due to the relaxation of the $z$-component of thermoelastic stresses and $d_{x y}$ that is fixed at its room-temperature value. The levels of the compressive stresses at a time of $1.59 \mathrm{~ns}$ after the laser pulse are $\sigma_{\mathrm{xx}}=\sigma_{\mathrm{yy}}=-0.6 \mathrm{GPa}$ on the $\mathrm{Cu}$ side of the lattice-mismatched interface and $\sigma_{\mathrm{xx}}=$ $\sigma_{\mathrm{yy}}=-1.3 \mathrm{GPa}$ on the Ag side, above the atomic mixing region. The difference in the levels of the compressive stresses can be explained by the larger thermal expansion coefficient of $\mathrm{Ag}$ 
compared to $\mathrm{Cu}$ and the slightly compressed initial state of the Ag film at $300 \mathrm{~K}$ (see section 3.2). The compressive stresses are also reflected in the pressure contour plot shown in Fig. 3.3b. The positive values of pressure, defined as a negative $1 / 3$ of the first invariant of the stress tensor, $\mathrm{P}=$ $-\left(\sigma_{\mathrm{xx}}+\sigma_{\mathrm{yy}}+\sigma_{\mathrm{zz}}\right) / 3$, contain contributions mainly from the first two diagonal components of the stress tensor, $\sigma_{\mathrm{xx}}$ and $\sigma_{\mathrm{yy}}$.

The distinct decrease in the value of $d_{z}$ upon the transition through the mixing region signifies the compression of the FCC lattice in the $z$ direction and can be interpreted as a transformation from FCC to BCC structure along the so-called Bain path [84]. Both crystal structures can be represented by a body centered tetragonal (BCT) structure with the ratio of BCT lattice constants $c / a=\sqrt{2}$ for a perfect FCC structure and $c / a=1$ for a perfect BCC structure, Fig. 3.6e. The change in the value of $d_{z}$ (and the corresponding BCT lattice parameter c) and the substitution of $\mathrm{Ag}$ atoms by "smaller" $\mathrm{Cu}$ atoms upon crossing the mixing region result in a gradual transformation of the structure from laterally compressed $\mathrm{FCC}$ structure of $\mathrm{Ag}$ to a BCC lattice composed of $\mathrm{Cu}$ atoms. The BCC structure is slightly compressed in $x$ and $y$ directions since the lattice parameter of the BCC structure is fixed at a value of $d_{x y}^{A g} \sqrt{2}=2.89 \AA$ and the lattice parameter of the metastable $\mathrm{BCC} \mathrm{Cu}$ predicted for a temperature of $800 \mathrm{~K}$ by the FBD EAM potential is $2.91 \AA$ (see below). The transition from the FCC Ag to the BCC Cu structure is apparent from the spatial variation of the $c / a$ ratio shown in Fig. 3.6d, where the values of the $c / a$ ratio that correspond to the perfect FCC and BCC lattices are marked by horizontal lines. The analysis of the local atomic structure $[79,80]$ also supports the conclusion on the BCC structure of the epitaxial layer located between the atomic mixing region and the displaced misfit interface. In the snapshots of the atomic configuration shown in Fig. 3.7b and $3.7 \mathrm{c}$, the atoms are colored according to the local atomic structure and most of the atoms in the epitaxial layer are identified as BCC atoms. 
(a-c) atomic configurations at $1.59 \mathrm{~ns}$ after the laser pulse

(a)

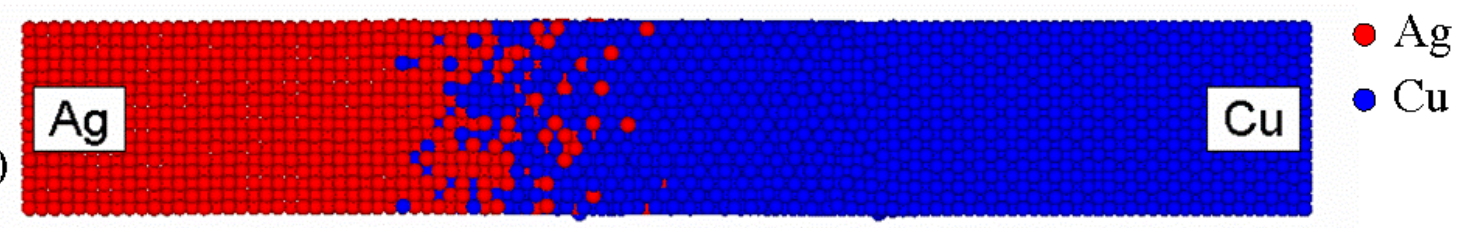

(b)

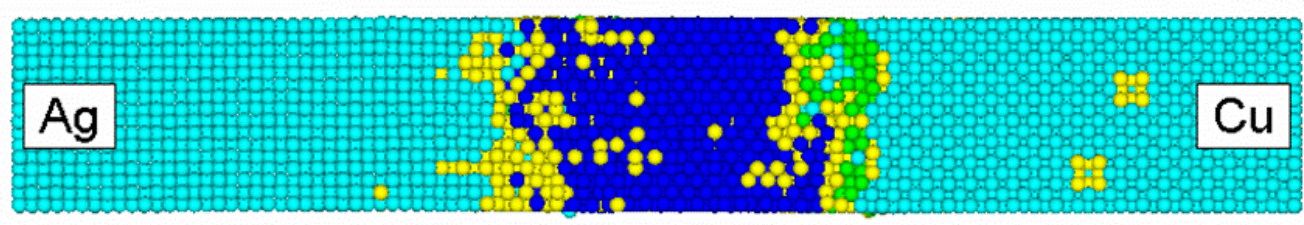

f fcc

o hop

- bcc

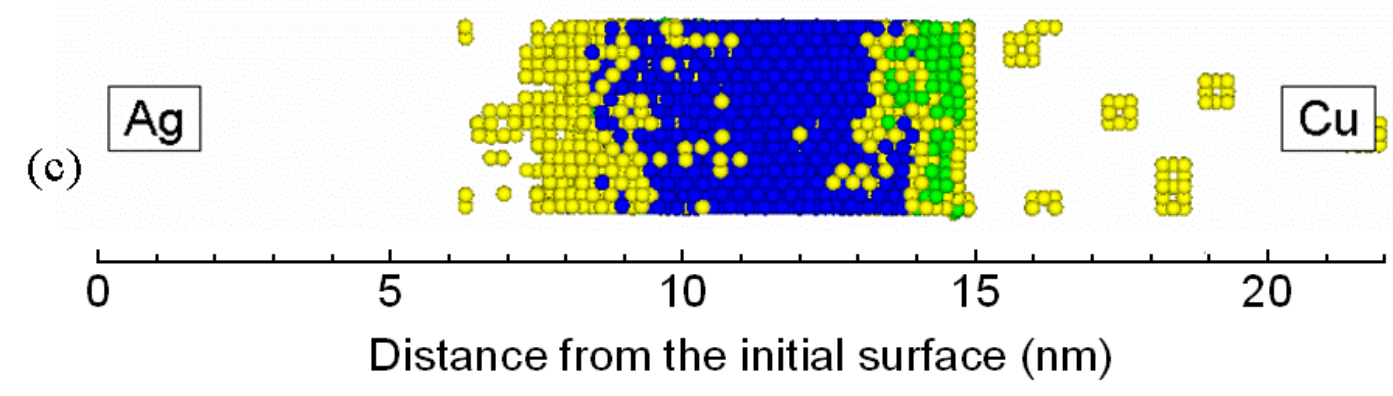

o defects

$(\mathrm{d}, \mathrm{e})$ after cooling of atomic configuration shown in (a-c)

(d)

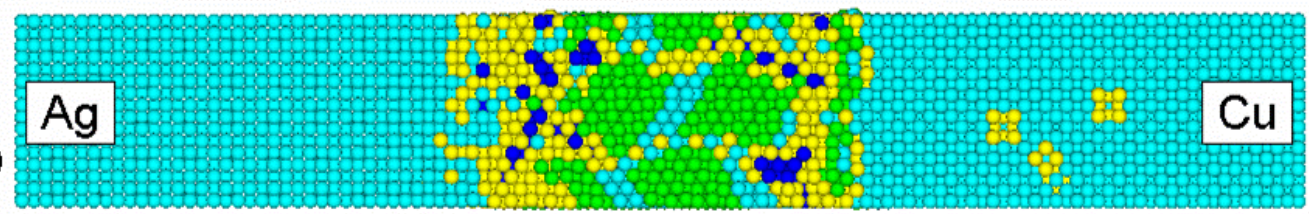

(e)

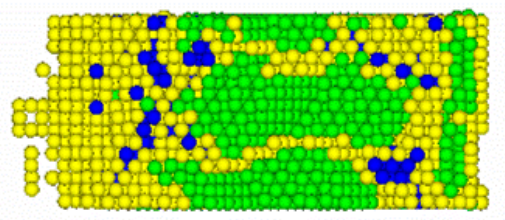

$\infty$

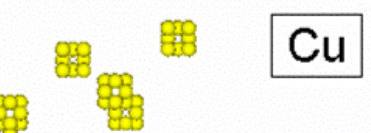

Figure 3.7: Snapshots of the atomic configurations in a region adjacent to the Ag-Cu interface. The atomic configuration shown in (a-c) is obtained by the end of the simulation (1.59 ns after the laser pulse) and quenched by applying the velocity dampening technique for $0.2 \mathrm{ps}$. The atomic configuration shown in (d) and (e) is obtained by a gradual cooling $(5 \mathrm{~K} / \mathrm{ps})$ of the system shown in (a-c). In (a), atoms are colored by their type: red for $\mathrm{Ag}$ and blue for $\mathrm{Cu}$. In (b-e), atoms are colored according to the output of a modified version [79] of the structural analysis of Ref. [80]: Light blue, green and blue atoms belong to local atomic configurations with FCC, $\mathrm{HCP}$ and $\mathrm{BCC} / \mathrm{BCT}$ structures, respectively; the structure around the yellow atoms cannot be 
assigned to any crystal structure and is characteristic of point defects and dislocation cores. The FCC atoms are blanked in (c) and (e) to expose the defect structure. The region shown in (a-c) corresponds to the region for which the distributions of structural and physical characteristics are shown in Fig. 3.6. The snapshots shown in (d) and (e) are aligned with the ones in (a-c) to match the location of the lattice-mismatched interface.

The observation of the BCC structure of the epitaxial layer can be related to the results of experimental [70,71] and computational [85] investigations of thin $\mathrm{Cu}$ films deposited on a $\mathrm{Ag}$ (001) substrate, where a pseudomorphic growth of BCC $\mathrm{Cu}$ is observed for film thicknesses of up to ten monolayers. A good match between the lattice parameter of the BCC Cu structure and the first-neighbor distance in the FCC Ag structure, combined with a relatively small energy difference between the FCC and BCC Cu structures (the difference in the cohesive energies of the two structures predicted with the FBD EAM potential is $22 \mathrm{meV} / \mathrm{atom}$, which is comparable to $20-40$ meV/atom predicted in ab initio calculations $[86,87]$ ) are the factors that facilitate the pseudomorphic epitaxial growth of the BCC Cu layer on the (001) face of the Ag crystal.

To further investigate the stability of $\mathrm{BCC} \mathrm{Cu}$, we performed two series of short (50 ps) simulations for a BCC system with the size of $25 \times 25 \times 25$ unit cells (31,250 atoms) and periodic or free boundary conditions applied in all directions. The simulations are performed for different values of temperature. In the case of the periodic boundary conditions, constant zero pressure is maintained during the simulations. We find that, when the periodic boundary conditions are applied, the BCC structure spontaneously transforms into a mixture of FCC and HCP closepacked structural regions at temperatures below $480 \mathrm{~K}$. Above $480 \mathrm{~K}$, the BCC structure remains stable for the duration of the simulations, $50 \mathrm{ps}$. With free boundary conditions, the BCC structure collapses into a mixture of FCC and HCP regions below $1000 \mathrm{~K}$ and remains stable above $1000 \mathrm{~K}$. Thus, the BCC structure exhibits an increased stability with increasing temperature. The melting temperature of $\mathrm{BCC} \mathrm{Cu}$, determined using the same liquid-crystal coexistence method discussed in section 2.5 for $\mathrm{FCC} \mathrm{Cu}$, yields the melting temperature of $1262 \pm 3 \mathrm{~K}$, more than $20 \mathrm{~K}$ below that of the FCC Cu structure. The lower value of the melting temperature indicates that, despite the increase in the stability of the $\mathrm{BCC} \mathrm{Cu}$ structure with increasing temperature, it remains a metastable non-equilibrium structure up to the melting temperature of the FBD EAM Cu material. 
In the Ag-Cu target irradiated by the laser pulse, the epitaxial $\mathrm{BCC} \mathrm{Cu}$ layer is stabilized by the presence of the underlying $\mathrm{Ag}$ (001) structure and remains stable during the cooling of the interfacial region down to $800 \mathrm{~K}$ (Fig. 3.4). The cooling rate in the combined TTM - MD simulation slows down with time and, to test the stability of the BCC structure upon further cooling more quickly, we performed a simulation of a faster cooling (with a rate of $5 \mathrm{~K} / \mathrm{ps}$ ) of the final atomic configuration obtained by the time of $1.59 \mathrm{~ns}$ in the TTM-MD simulation. In the simulation of the fast cooling, the BCC structure of the epitaxial layer remains stable down to the temperature of $300 \mathrm{~K}$ but transforms to a close packed structure characterized by a mixture of HCP and FCC structural regions upon further cooling, Fig. 3.7d and 3.7e.

\subsubsection{Structure of the runaway lattice-mismatched interface}

Detailed analysis (see Appendix B) of the interface separating the pseudomorphic epitaxial layer of $\mathrm{BCC} \mathrm{Cu}$ from the rest of the $\mathrm{Cu}$ substrate reveals a corrugated structure that has a thickness of $\sim 1.6 \mathrm{~nm}$ and is more complex compared to the plane array of $\frac{1}{2}\langle 110\rangle$ misfit dislocations shown in Fig. 3.2b for the original sharp semicoherent $\mathrm{Ag}-\mathrm{Cu}$ interface. The atomic structure of the interface is shown in Fig. 3.8, where atoms are colored according to the local atomic structure and FCC atoms are blanked to expose the structure of the interface. The interface consists of a periodic array of stacking fault pyramids outlined by stair-rod partial dislocations [88], with the lateral periodicity of the array matching that of the original semicoherent interface, Fig. 3.2b. A schematic representation of a pyramid is shown in Fig. 3.8b. The partial dislocations in the base of each pyramid are identified as Lomer-Cottrell locks with $\vec{b}=\frac{1}{6}\langle 110\rangle$ and the partial dislocations forming the edges of the pyramids are Hirth locks with $\vec{b}=\frac{1}{3}\langle 010\rangle$. The faces of the pyramids are intrinsic stacking faults that show up as the layers of HCP atoms in Fig. 3.8a. 


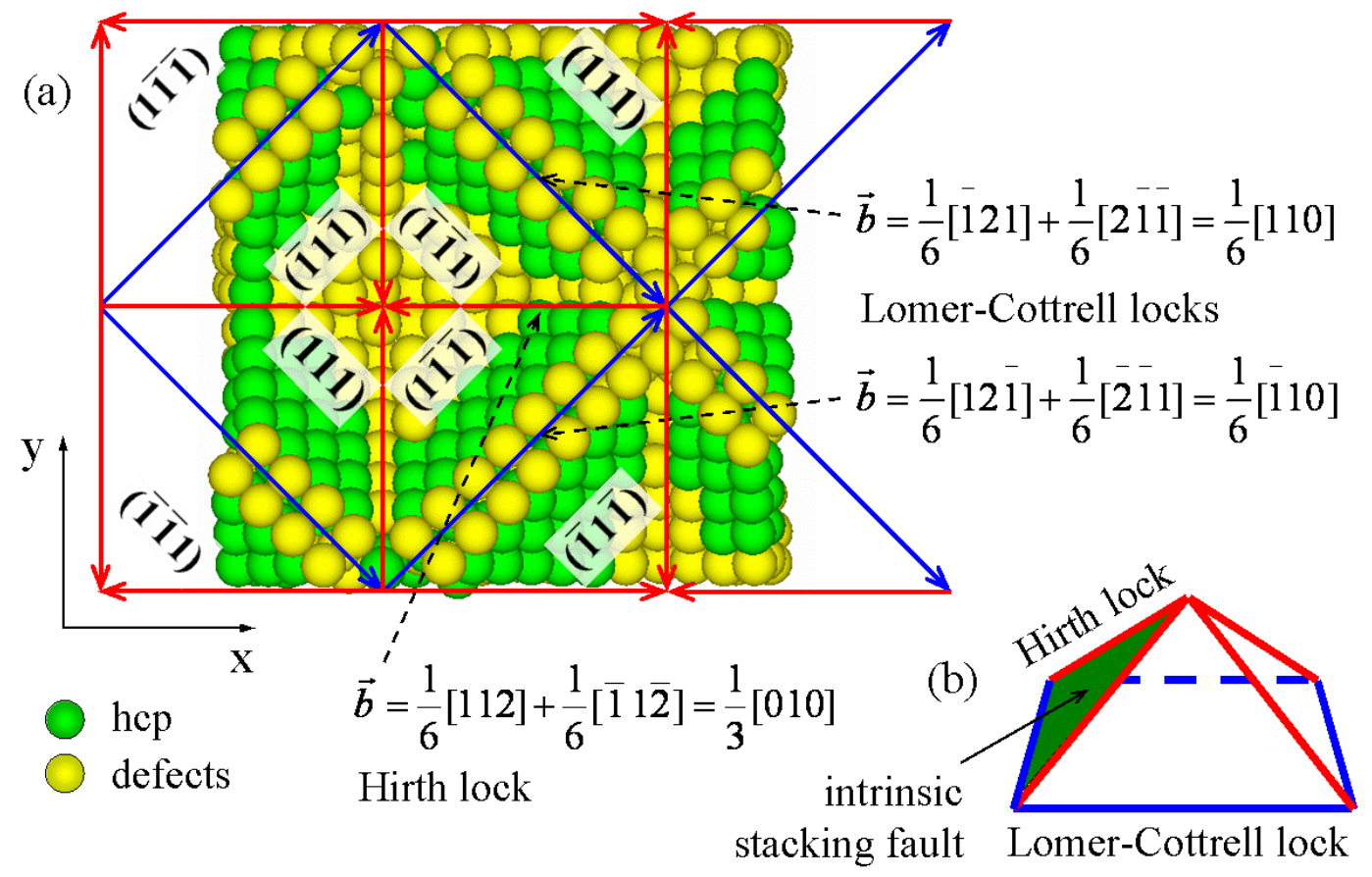

Figure 3.8: Structure of the "runaway" lattice-mismatched interface generated in the $\mathrm{Ag}-\mathrm{Cu}$ system irradiated by a $200 \mathrm{fs}$ laser pulse. The interface is located in the $\mathrm{Cu}$ substrate, below the $\mathrm{Ag}-\mathrm{Cu}$ mixing region and the view from the side of the $\mathrm{Cu}$ substrate is shown in (a). Similarly to Fig. 3.7c, atoms are colored according to the local atomic structure, with green atoms located within the planes of intrinsic stacking faults in the FCC structure (local HCP configurations), yellow atoms cannot be assigned to any crystal structure and belong to the dislocation cores, and FCC atoms are blanked to expose the structure of the interface. The structure of the interface is represented by an array of stacking fault pyramids outlined by stair-rod partial dislocations. The lines of stair-rod partial dislocations in the base and at the edges of the pyramids (Lomer-Cottrell and Hirth locks, respectively) are shown by blue and red arrows. Examples of the dislocation reactions responsible for the formation of the locks are shown in the figure. A schematic representation of a structural element of the interfacial structure is shown in (b).

The interfacial structure consisting of the stacking fault pyramids can be considered as a product of dissociation of the original grid of perfect $\vec{b}=\frac{1}{2}\langle 110\rangle$ dislocations shown in Fig. 3.2b. The corresponding dislocation reactions can be illustrated by considering a perfect dislocation with $\vec{b}=\frac{1}{2}[110]$ and dislocation line oriented along the [110] direction (intersection of (111) 
and (111) crystallographic planes). This dislocation can split into two perfect dislocations, $\frac{1}{2}[110]=\frac{1}{2}[011]+\frac{1}{2}[10 \overline{1}]$, with the corresponding slip planes of (111) and (111). These two dislocations can dissociate into two partial dislocations by the following reactions: $\frac{1}{2}[011]=\frac{1}{6}[112]+\frac{1}{6}[\overline{121}]$ in the (111) plane and $\frac{1}{2}[10 \overline{1}]=\frac{1}{6}[2 \overline{1} \overline{1}]+\frac{1}{6}[11 \overline{2}]$ in the (111) plane. Then $\frac{1}{6}[121]$ can react with $\frac{1}{6}[211]$ to generate a Lomer-Cottrell lock at the intersection [110] of planes (111) and (111) through the reaction $\frac{1}{6}[121]+\frac{1}{6}[211]=\frac{1}{6}[110]$ shown in Fig. 3.8a. Similarly, a Lomer-Cottrell lock at the intersection [110] of planes (111) and (111) can be generated through the reaction $\frac{1}{6}[12 \overline{1}]+\frac{1}{6}[\overline{2}-\overline{1}]=\frac{1}{6}[\overline{110}]$, etc. As an example of the generation of Hirth locks along the edges of the pyramids, a reaction $\frac{1}{6}[112]+\frac{1}{6}[\overline{1} 1 \overline{2}]=\frac{1}{3}[010]$ producing a stair-rod partial dislocation at the intersection [101] of planes (111) and(111) is also shown in Fig. 3.8a.

The formation of continuous stacking fault interfacial structures similar to the ones described above has been suggested in Ref. [89] for $\mathrm{Cu}-\mathrm{Ni}$ interfaces subjected to tensile stresses in the plane of the interface. The results of Monte Carlo simulations performed for $\mathrm{Ag}-\mathrm{Cu}$ interfaces $[90,91]$ also suggest that the (001) $\mathrm{Ag}-\mathrm{Cu}$ interface is unstable against decomposition into $\{111\}$ facets, which leads to the formation of $\mathrm{Ag}$-rich pyramidal protrusions into the $\mathrm{Cu}$-rich phase. The dissociation of the interface into the stacking fault pyramids creates a strong barrier to slip [89] and results in the effective hardening of the interfacial region.

\subsection{Summary}

The atomic mixing and structural transformations in a $\mathrm{Ag}$ film $-\mathrm{Cu}$ substrate system irradiated by a femtosecond laser pulse were investigated with a computational model that combines the classical MD method with a continuum description of laser excitation, electronphonon equilibration, and electron heat conduction. The initial energy redistribution and the location/depth of the region undergoing melting and resolidification are strongly affected by the 
difference in the strength of electron-phonon coupling of the film and substrate materials and by the transient variations of the electron-phonon coupling related to the thermal excitation of lower band electrons. The higher strength of the electron-phonon coupling in $\mathrm{Cu}$ compared to $\mathrm{Ag}$ results in a preferential sub-surface heating and melting of the Cu substrate.

The steep temperature gradient and fast electronic heat conduction in $\mathrm{Cu}$ result in a very high cooling rate, up to $\sim 3 \times 10^{11} \mathrm{~K} / \mathrm{s}$, and create the conditions of strong undercooling in the melted region. Although the velocity of epitaxial regrowth of the $\mathrm{Cu}$ substrate reaches its maximum value of $\sim 100 \mathrm{~m} / \mathrm{s}$, the complete resolidification of the melted part of the target takes almost a nanosecond and the temperature of the interfacial region drops to values that are close to the eutectic temperature of the $\mathrm{Ag}-\mathrm{Cu}$ system. At these temperatures the second resolidification front starts to propagate from the side of the Ag film, crosses the mixing region and meets the first resolidification front moving from the $\mathrm{Cu}$ side. As a result of this resolidification process, the new lattice-mismatched interface is separated from the Ag-Cu mixing region and shifted into the $C u$ substrate.

Detailed analysis of the structure of the resolidified part of the layered target indicates that the $\mathrm{Ag}-\mathrm{Cu}$ mixing region is separated from the new lattice-mismatched interface by an intermediate $2 \mathrm{~nm}$ wide pseudomorphic $\mathrm{BCC}$ Cu layer that grows epitaxially from the (001) face of the FCC Ag film during the final stage of the resolidification process. The latticemismatched interface that separates the pseudomorphic epitaxial $\mathrm{BCC} \mathrm{Cu}$ from the rest of the $\mathrm{Cu}$ substrate is found to have a complex three-dimensional corrugated structure consisting of a periodic array of stacking fault pyramids outlined by stair-rod partial dislocations (LomerCottrell locks in the base of the pyramids and Hirth locks at the edges of the pyramids). The intermediate BCC layer and the stacking fault pyramid structure of the "runaway" mismatched interface are likely to present a strong barrier for dislocation propagation, resulting in the effective hardening of the layered structure treated by the laser irradiation.

The concentration profiles in the atomic mixing region generated as a result of laserinduced melting and resolidification are more than 5 times wider compared to the width of the equilibrium interface in this immiscible system. Moreover, the preferential melting of the $\mathrm{Cu}$ substrate creates the conditions for the formation of asymmetric concentration profiles, with a 
more extensive diffusion of $\mathrm{Ag}$ atoms into the $\mathrm{Cu}$ substrate compared to the diffusion of $\mathrm{Cu}$ atoms into the Ag film. 


\section{4. $\mathrm{BCC} \mathrm{Cu}$ formation and its stability}

\subsection{Introduction}

The results of the simulations of femtosecond laser irradiation on $\mathrm{Ag}$ film - $\mathrm{Cu}$ substrate system presented in the previous section, demonstrated that an intermediate metastable $\mathrm{BCC} \mathrm{Cu}$ layer can be formed through the pseudomorphic epitaxial growth on (001) Ag face. In fact, metastable $\mathrm{BCC} \mathrm{Cu}$ (its formation and stability) is a subject of fundamental scientific interest by itself. Many efforts have been devoted to finding ways to prepare and stabilize the $\mathrm{BCC} \mathrm{Cu}$. The experimental epitaxial growth of thin (up to $100 \AA$ ) films of $\mathrm{BCC} \mathrm{Cu}$ on $\mathrm{Ag}\{001\}$ was firstly reported in 1977 [92]. In a subsequent study, Smith et al. [93] argued, from the plot of Auger signal against evaporation time, that the first three atomic layers of $\mathrm{Cu}$ deposited on $\mathrm{Ag}$ $\{001\}$ are possibly pseudomorphic, but not thicker layers. More recent experimental [70,71] and computational [85] investigations have found the pseudomorphic growth of $\mathrm{BCC} \mathrm{Cu}$ for film thicknesses of up to ten monolayers. Further deposition causes the surface to become buckled and leads to the transition to the FCC structure. Besides the Ag $\{001\}$ substrate, islands of BCC $\mathrm{Cu}$ are found to grow on BCC Fe $\{001\}$ [94] due to the lattice match (lattice constant of $\sim 2.87 \AA$ for both). In one word, there is plenty of evidence of $\mathrm{BCC} \mathrm{Cu}$ formation through epitaxial growth that supports our observation in the previous section. But to the best of our knowledge, nobody ever successfully stabilizes the BCC $\mathrm{Cu}$ beyond several monolayers.

Motivated by these intriguing results reported in the previous section, two series of simulations are performed to explore if it is possible to generate a thicker layer of $\mathrm{BCC} \mathrm{Cu}$ structure that is "stable" at the room temperature by using short pulse laser processing. The first series of simulations, reported in section 4.2, are on the short pulse laser irradiation of a $\mathrm{Cu}$ film - Ag substrate system. The second series of simulations, discussed in section 4.3, are on the short pulse laser irradiation of a sandwiched $\mathrm{Ag}$ film $-\mathrm{Cu}$ film $-\mathrm{Ag}$ substrate system. The conclusions drawn from these simulations are presented in section 4.4.

The computational setup used in these simulations is similar to the one used for the $\mathrm{Au}$ film - Cu system in Ref. [44] and the Ag film - Cu substrate system in Ref. [55] and the previous section. The setups used for $\mathrm{Cu}$ film - Ag substrate system and Ag film - Cu film - Ag substrate system are schematically illustrated in Figs. $4.1 \mathrm{a}$ and $4.1 \mathrm{~b}$ respectively. The films and the 
substrate have the same FCC structure and [001] crystallographic orientation with respect to the surface before laser irradiation. The effective laser energy deposition depth is assumed to be 30 $\mathrm{nm}$ in these simulations.
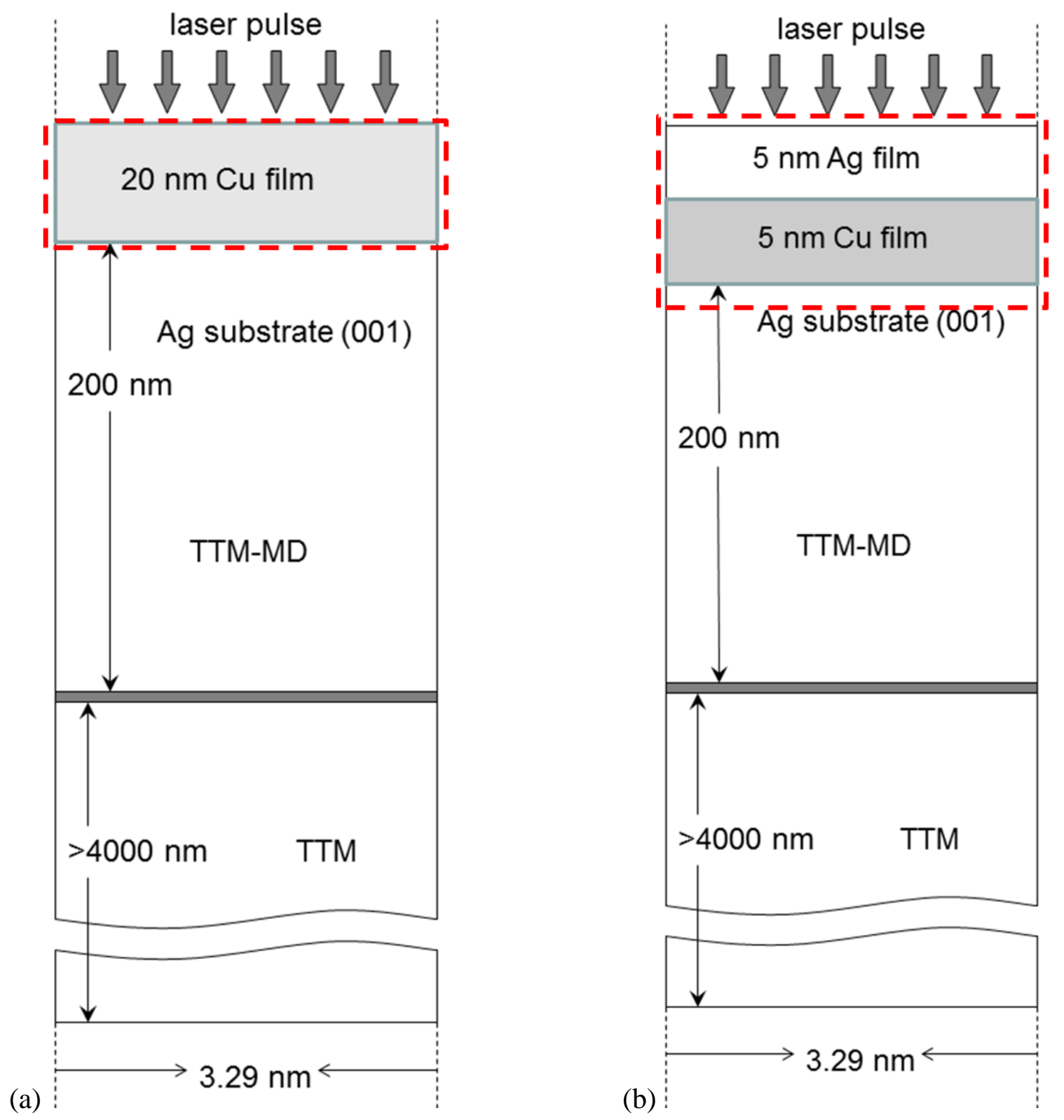

Figure 4.1: Schematic sketch of the simulation setups used in the exploration of the generation of BCC Cu by short pulse laser processing of: (a) $20 \mathrm{~nm} \mathrm{Cu}$ film - Ag substrate system. (b) $5 \mathrm{~nm}$ Ag film $-5 \mathrm{~nm} \mathrm{Cu}$ film - Ag substrate system. TTM-MD model is applied in the surface part of the target, whereas the electron heat conduction in the deeper part of the substrate is simulated with the conventional TTM. The desired melting region is marked by dashed squares. 


\subsection{0 fs laser irradiation of $20 \mathrm{~nm} \mathrm{Cu}$ film $-\mathrm{Ag}(001)$ substrate system}

The computational setup is shown in Fig. 4.1a. The idea of these simulations is simple. Short pulse laser irradiation leads to the melting the whole $\mathrm{Cu}$ film and a thin layer of $\mathrm{Ag}$ substrate as marked by red dashed squares in Fig. 4.1a. Rapid resolidifcation from the Ag substrate can then be expected to proceed through the epitaxial growth of $\mathrm{BCC} \mathrm{Cu}$ on $\mathrm{Ag}(001)$ due to the fast cooling. Thus the first step is to find a threshold fluence for melting the whole $\mathrm{Cu}$ film and a thin layer of Ag substrate. Then we focus on a single simulation performed at this particular fluence to study the resolidification process, $\mathrm{BCC} \mathrm{Cu}$ growth and its stability. The reason we want to melt only a thin layer of $\mathrm{Ag}$ substrate is two-fold. First, more melting of $\mathrm{Ag}$ substrate requires the laser irradiation at higher laser fluence, which can generate the stronger unloading tensile pressure wave that propagates through the $\mathrm{Cu}-\mathrm{Ag}$ interface and may lead to the spallation of the $\mathrm{Cu}$ film as seen in Fig. 4.2c. Second, the more of the Ag substrate is melted, the more time the melted $\mathrm{Cu}$ needs to wait for the epitaxial growth. During the long waiting time, the temperature of the melted $\mathrm{Cu}$ can drop down far below its melting temperature due to the fast cooling. Under the condition of strong undercooling, the homogeneous nucleation of the closed packed structure (FCC/HCP) may take place in the melted $\mathrm{Cu}$, which would inhibit the epitaxial growth of $\mathrm{Cu}$ on $\mathrm{Ag}$ (001) face.

\subsubsection{Threshold fluence for melting of the whole $\mathrm{Cu}$ film}

Three representative simulations with different adsorbed fluences, $F=0.03 \mathrm{~J} / \mathrm{cm}^{2}, 0.04$ $\mathrm{J} / \mathrm{cm}^{2}$, and $0.05 \mathrm{~J} / \mathrm{cm}^{2}$ have been performed and illustrated in Fig. 4.2. Due to the much stronger electron-phonon coupling of $\mathrm{Cu}$ as compared to $\mathrm{Ag}$ [45,54], the heating is localized in the $\mathrm{Cu}$ film. At the low fluence $\mathrm{F}=0.03 \mathrm{~J} / \mathrm{cm}^{2}$, only a very thin $\mathrm{Cu}$ layer near free surface is melted. At the high fluence $\mathrm{F}=0.05 \mathrm{~J} / \mathrm{cm}^{2}$, the $\mathrm{Cu}$ film is spallated from the Ag substrate due to the interaction of unloading tensile pressure wave with the $\mathrm{Cu}-\mathrm{Ag}$ interface. The fluence of $\mathrm{F}=$ $0.04 \mathrm{~J} / \mathrm{cm}^{2}$ is exactly what we need, as the whole $\mathrm{Cu}$ film and the thin layer of Ag substrate near the interface are melted, and no spallation is observed. In the following subsections, we focus on the simulation at $\mathrm{F}=0.04 \mathrm{~J} / \mathrm{cm}^{2}$ to study the resolidification process, $\mathrm{BCC} \mathrm{Cu}$ growth and its stability. 
(a)
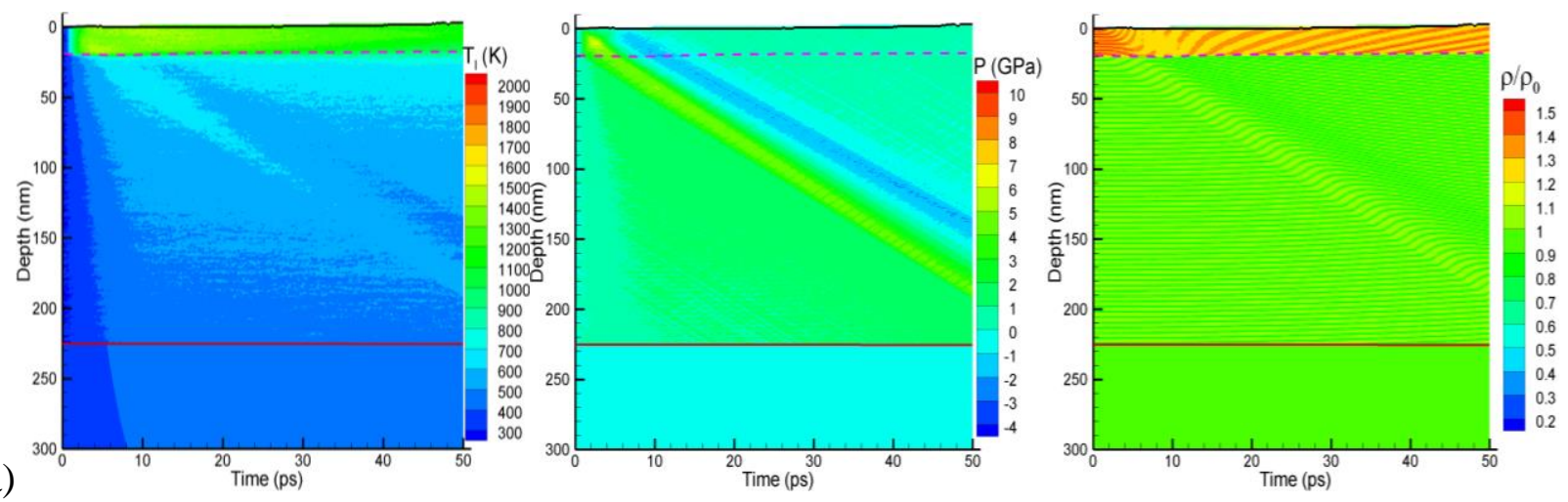

(b)
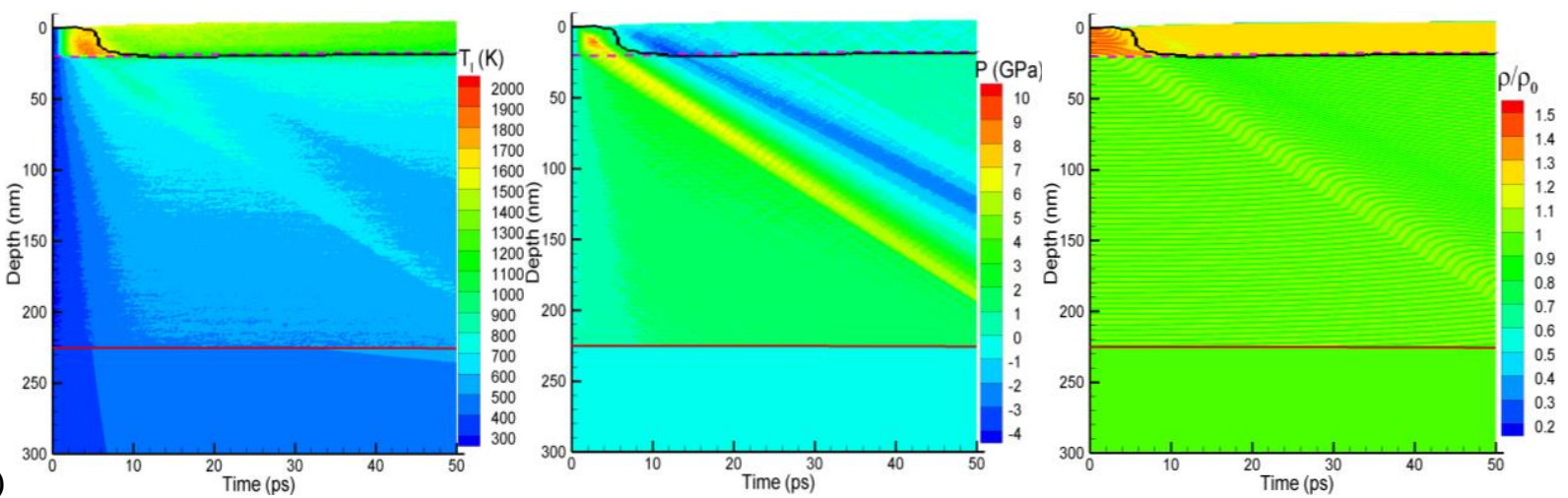

(c)
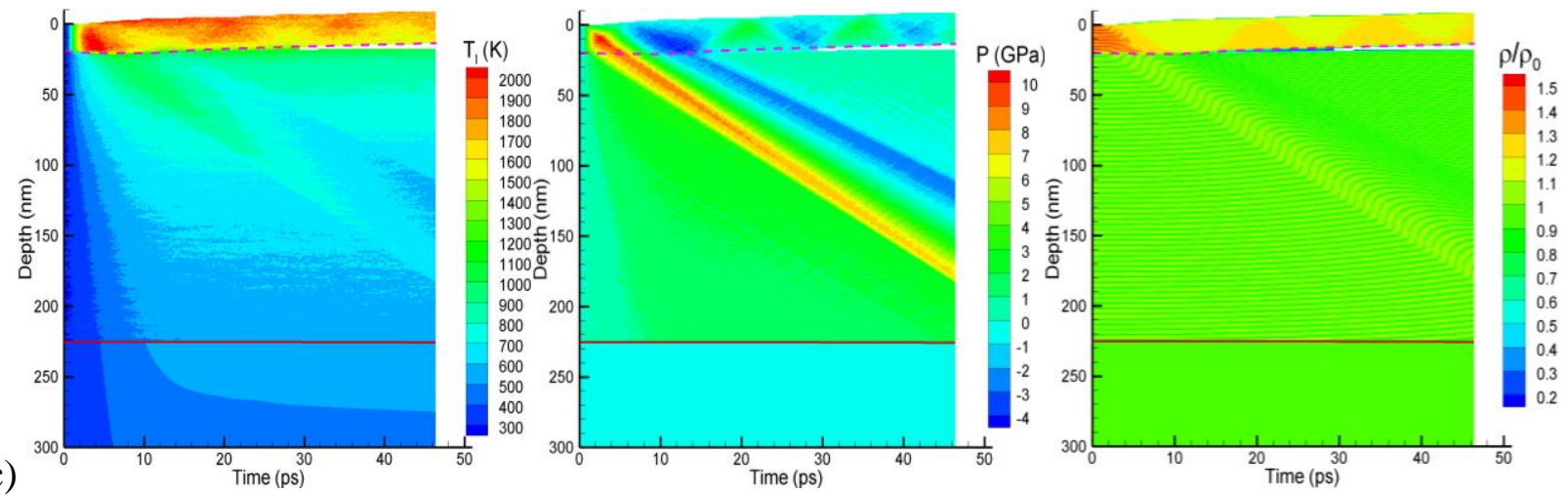

Figure 4.2: Contour plots of the lattice temperature, pressure and density (normalized to the density of substrate $\mathrm{Ag}$ at $300 \mathrm{~K}$ before the irradiation) as a function of time and depth for simulations of $\mathrm{Cu}$ film - Ag substrate systems irradiated with $200 \mathrm{fs}$ laser pulses at fluences of (a) $0.03 \mathrm{~J} / \mathrm{cm}^{2}$, (b) $0.04 \mathrm{~J} / \mathrm{cm}^{2}$ and (c) $0.05 \mathrm{~J} / \mathrm{cm}^{2}$. Laser pulse is directed along the Y-axis, from the top of the contour plots. The black lines separate the melted regions from the crystalline bulk of the target. Purple dashed lines indicate the $\mathrm{Cu}-\mathrm{Ag}$ interface. Red lines separate the MD and continuum parts of the combined TTM-MD model. The $\mathrm{Cu}$ film is spallated at the laser fluence 
of $0.05 \mathrm{~J} / \mathrm{cm}^{2}$ as seen in c). The density scale in (c) is normalized to the density of substrate Ag before the irradiation (at $300 \mathrm{~K}$ ), $\rho_{0}$.

\subsubsection{Epitaxial growth of BCC Cu on Ag (001) face during the resolidification}

The contour plots, describing the temperature, pressure, density and liquid fraction evolution up to $3.43 \mathrm{~ns}$, are shown in Fig. 4.3 for the simulation of of the laser irradiation of 20 $\mathrm{nm} \mathrm{Cu}$ film - Ag substrate system at the particular laser fluence $\mathrm{F}=0.04 \mathrm{~J} / \mathrm{cm}^{2}$,. To identify the liquid and crystal regions which are separated by the black lines in the contour plots of Fig. 4.3, the local order parameter suggested in Ref [63] is calculated for each atom with respect to the original FCC structure. The local order parameter changes from 0 to 1 with a value of 0.04 chosen to distinguish between the "liquid" and crystal structures, i.e., the atom with local order parameter less than 0.04 is identified as "liquid" atom, otherwise it is crystal atom. The "liquid" fraction in Fig. 4.3d is thus calculated as concentration of the identified "liquid" atoms with local order parameter less than 0.04 for each layer of $1 \mathrm{~nm}$. The "liquid" region is defined as the region where the "liquid" fraction is greater than 10. However, the local order parameter is calculated with respect to the original FCC structure, thus the crystal atoms with different orientation may be counted as "liquid" atoms as shown in Fig. 4.3d. As discussed below in section 4.2.3, the misinterpretation of crystal atoms by local order parameter may be useful, since it can help detect the structural transformations.

As seen in Fig. 4.3a, the strong temperature gradient and the fast electronic heat conduction lead to the rapid cooling of the surface region of the irradiated target. By the time of $100 \mathrm{ps}$, the temperature in the whole melted region drops below the equilibrium melting temperature of $\mathrm{Cu}$ and the movement of the resolidification front (black lines) from the $\mathrm{Ag}$ substrate is activated. 
(a)

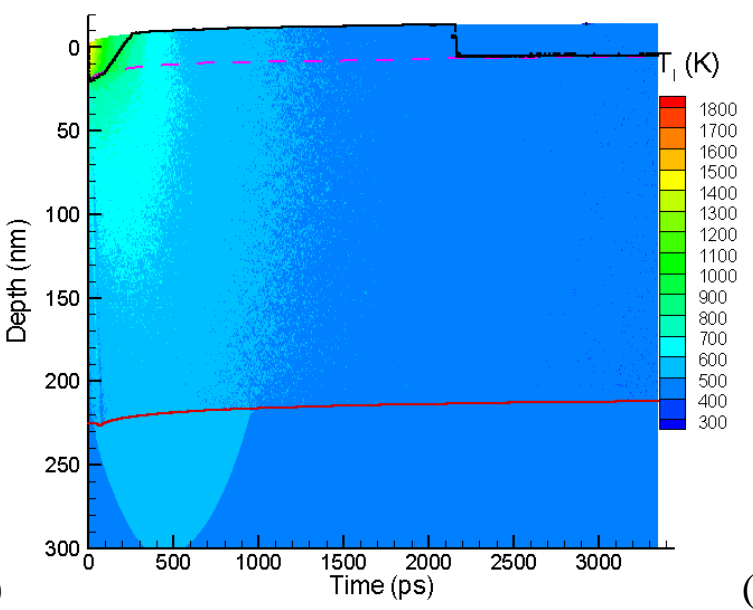

(b)

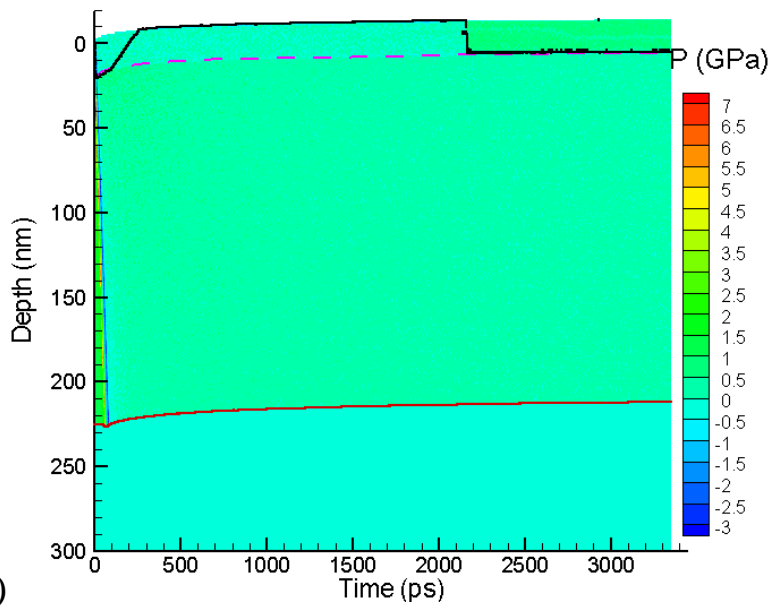

(c)

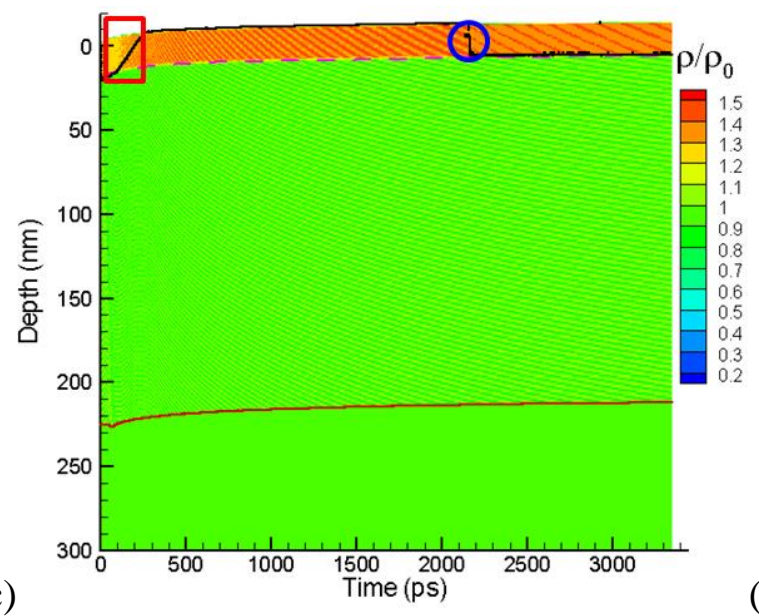

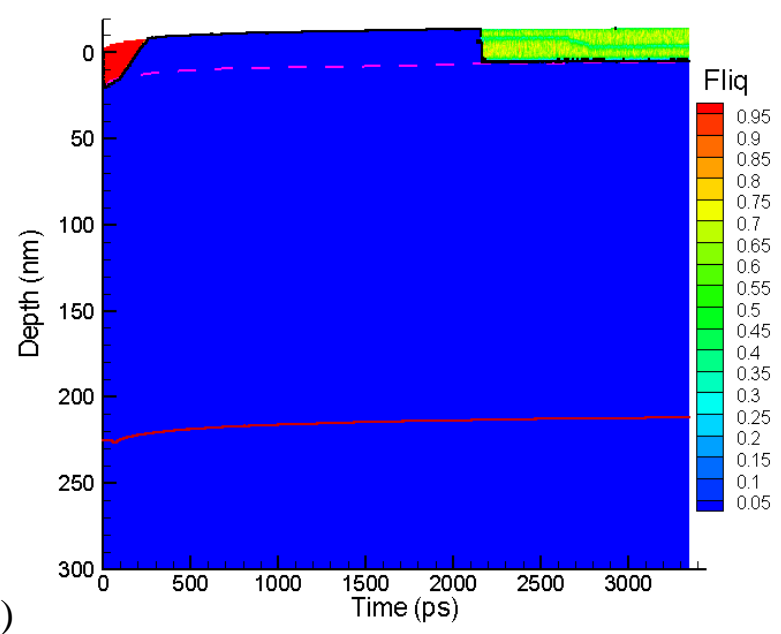

Figure 4.3: Contour plots describing the evolution of (a) lattice temperature a), (b) pressure, (c) density and (d) liquid fraction in the simulation of $\mathrm{Cu}$ film - Ag substrate systems irradiated with $200 \mathrm{fs}$ laser pulse at absorbed fluences $\mathrm{F}=0.04 \mathrm{~J} / \mathrm{cm}^{2}$. The black lines separate the melted regions from the crystalline bulk of the target, while the red lines separate the MD and continuum parts of the combined TTM-MD model. The purple dashed lines mark the $\mathrm{Cu}-\mathrm{Ag}$ interface. The red rectangle and the blue circle in (c) show the region and time interval for which snapshots are shown in Fig. 4.4 and Fig. 4.5. The liquid fraction is obtained based on the calculation of local order parameter [63] as discussed in the text.

As expected, the propagation of the resolidification into the $\mathrm{Cu}$ film leads to the pseudomorphic epitaxial growth of $\mathrm{Cu}$ film on $\mathrm{Ag}$ (001) substrate, as shown in Fig. 4.4a. Structural analysis performed with a method suggested in Ref. $[79,80]$ verified the BCC structure 
of the $\mathrm{Cu}$ film as shown in Fig. 4.4b. The whole resolidfication takes $\sim 150 \mathrm{ps,}$ which corresponds to the velocity of the resolidification front of $\sim 130 \mathrm{~m} / \mathrm{s}$.

\section{$\mathrm{Cu}$ film Ag substrate}
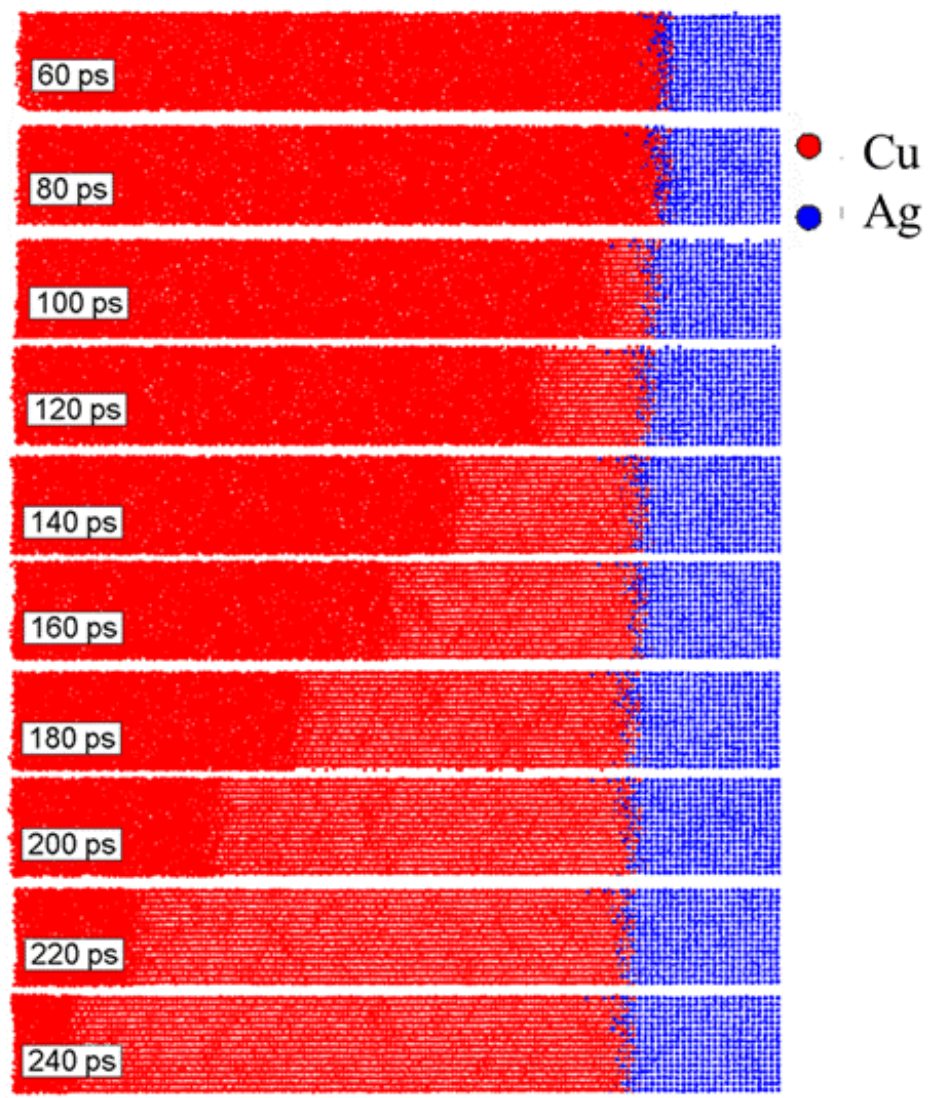

(a)

\section{$260 \mathrm{ps}$}

(b)

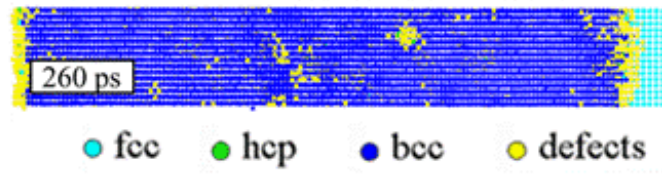

Figure 4.4: Snapshots taken for the region and the time interval marked in Fig. 4.3c by the red rectangle. Atoms are colored according to their type in (a) - Ag atoms are colored blue and $\mathrm{Cu}$ atoms are colored red. Atoms are colored by their local structural environment in (b), where a snapshot for the time of $260 \mathrm{ps}$ is shown. To reduce the thermal noise, the atomic configuration is quenched for $0.2 \mathrm{ps}$ before the structural analysis is performed to obtain (b). 


\subsubsection{Stability of the generated BCC Cu film}

As seen in the contour plots shown in Fig. 4.3 (resolidification front and liquid fraction), the $\mathrm{BCC} \mathrm{Cu}$ film stays stable up to $2157 \mathrm{ps}$. When the lattice temperature drops down to $\sim 450 \mathrm{~K}$, the BCC Cu film collapses down to a mixture of FCC and HCP structural regions as seen in Fig. 4.5. Since the local order parameter, used to identify the crystal/liquid regions, is calculated in reference to the original FCC structure, the mixture of FCC and HCP is partly identified as "liquid" phase due to its misorientation compared to the original FCC structure. That is why we see the larger "liquid fraction" in Fig. 4.3d after $\mathrm{BCC} \mathrm{Cu}$ transforms into close-packed structrures. This transformation is an extremely fast process that takes only $\sim 15 \mathrm{ps}$, corresponding to a high effective speed of $\sim 1400 \mathrm{~m} / \mathrm{s}$. This high speed indicates that the transformation is a combination of heterogeneous and homogeneous processes, as seen in the snapshots shown in Fig. 4.5. At 2158 ps, the first close-packed structure is nucleated in the BCC $\mathrm{Cu}$ film near the free surface and grows quickly. At 2167 ps, the second new close-packed structure with different orientation is nucleated in $\mathrm{BCC} \mathrm{Cu}$ near the $\mathrm{Ag}-\mathrm{Cu}$ interface and it quickly grows up to the $\mathrm{Ag}-\mathrm{Cu}$ interface. The second close-packed structure is then consumed during the further growth of the first one. Finally, the whole $\mathrm{Cu}$ film is closely packed oriented in the direction of the first nucleated close-packed structure. In summary, we see from this simulation that a single coherent interface with FCC Ag (001) cannot hold the BCC Cu film down to the room temperature. 


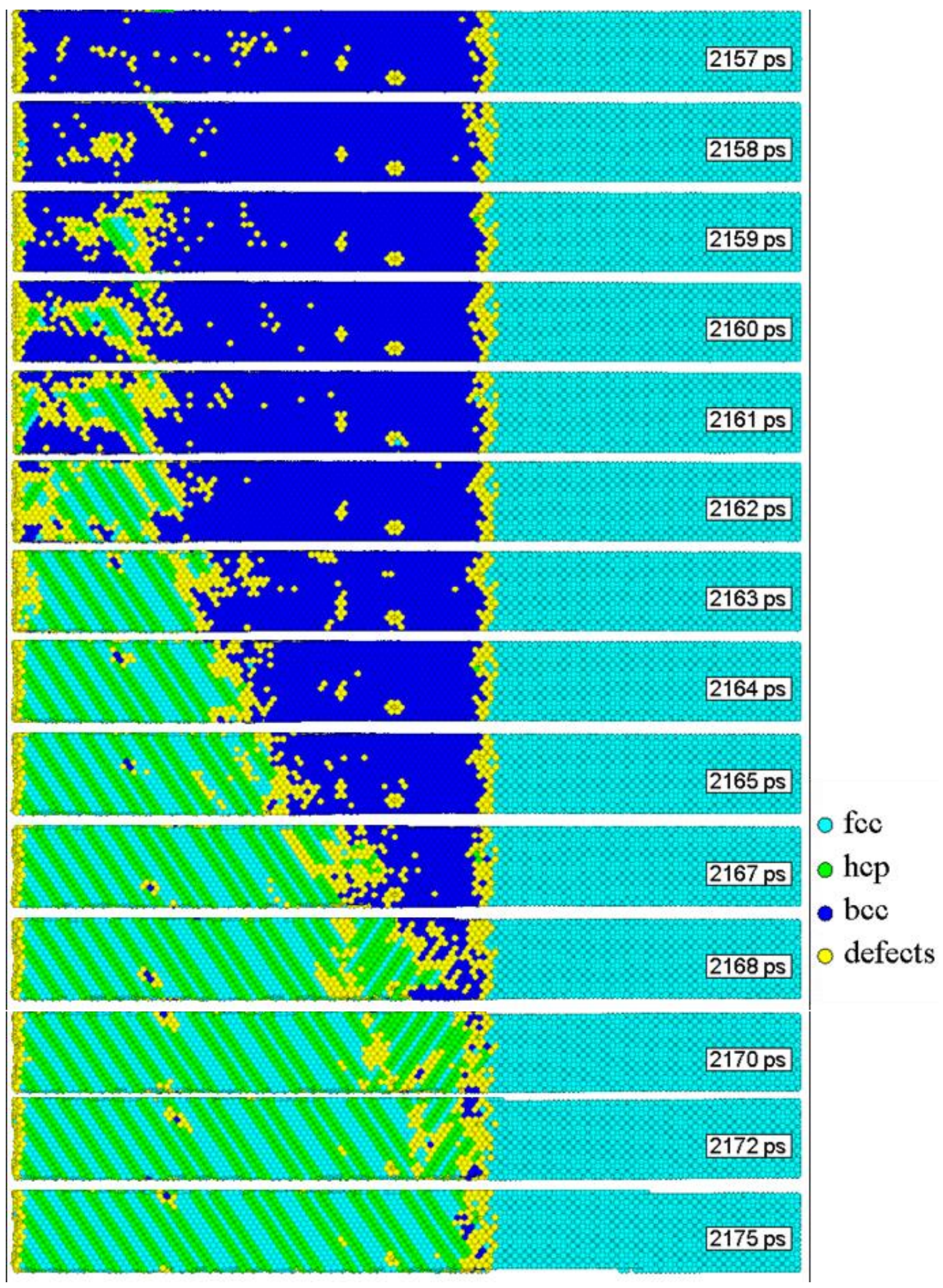

Figure 4.5: Snapshots taken for the region and the time interval marked in Fig. 4.3c by the blue circle. Atoms are colored according to their local structural environment. To reduce the thermal noise, the system is quenched for 0.2 ps before the structure analysis. 


\subsection{0 fs laser irradiation of $5 \mathrm{~nm} \mathrm{Ag} \mathrm{film}-5 \mathrm{~nm} \mathrm{Cu}$ film $-\mathrm{Ag}(001)$ substrate system}

From the simulations on $\mathrm{Cu}$ film - $\mathrm{Ag}$ (001) substrate system discussed above in section 4.2, we observe that a BCC Cu layer grows epitaxially on $\mathrm{Ag}$ (001) after short pulse laser irradiation, but becomes unstable upon cooling down to the room temperature. Naturally, we come up with an idea of simulations of short pulse laser irradiation of a $\mathrm{Ag}$ film $-\mathrm{Cu}$ film $-\mathrm{Ag}$ (001) substrate system. With a proper choice of laser fluence, short pulse laser irradiation can melt the whole top $\mathrm{Ag}$ film, $\mathrm{Cu}$ film and a thin layer of $\mathrm{Ag}$ substrate as marked by red dashed squares in Fig. 4.1b. Then, we expect the resolidification from the Ag substrate to result in the epitaxial growth of BCC Cu on FCC Ag (001) and the subsequent epitaxial growth of FCC Ag on $\mathrm{BCC} \mathrm{Cu}(001)$ due to the fast cooling. As a result, we expect to form $\mathrm{BCC} \mathrm{Cu}$ sandwiched by FCC Ag (001) on both sides, and it is really interesting to study the stability of BCC $\mathrm{Cu}$ under this condition.

The choice of the very "thin" films, e.g. $5 \mathrm{~nm} \mathrm{Ag}$ film - $5 \mathrm{~nm} \mathrm{Cu}$ film, is related to our goal of melting of the whole two films without spallation at the interface. For the system with the thicker films, e.g. $15 \mathrm{~nm} \mathrm{Ag} \mathrm{film}-20 \mathrm{~nm} \mathrm{Cu}$ film, we observe spallation at fluences below the threshold for the complete melting of the two films, as seen in Fig. 4.6. From the lattice temperature contour plot, we can see that the heating is localized in the intermediate $\mathrm{Cu}$ film due to the much stronger electron-phonon coupling in $\mathrm{Cu}$. In other words, the intermediate $\mathrm{Cu}$ film acts as the heat sink for the surrounding Ag. To melt a "thick" top Ag film, a high laser fluence is required, which leads to a higher temperature in the intermediate $\mathrm{Cu}$ film and the weakening of the interfaces. That is why the spallation occurs at the interfaces before the complete melting of the top two films can be achieved. The choice of the system with very "thin" films, especially the thin top Ag film, can overcome this problem. First, it is easy to melt the two "thin" films at a relatively low fluence. Second, the unloading tensile pressure at the interface is weak when the film is thin due to the stress relaxation near the free surface and also the fact that the strength of the unloading tensile wave builds up along the wave propagation. As a result, the spallation of the film at the interface can be avoided for thin $\mathrm{Ag}$ and $\mathrm{Cu}$ films. In this section, we choose the system of $5 \mathrm{~nm} \mathrm{Ag}$ film - $5 \mathrm{~nm} \mathrm{Cu}$ film on $\mathrm{Ag}$ (001) substrate as an example to achieve the complete melting of the two films without spallation at the interfaces during the short pulse laser irradiation. 
(a)
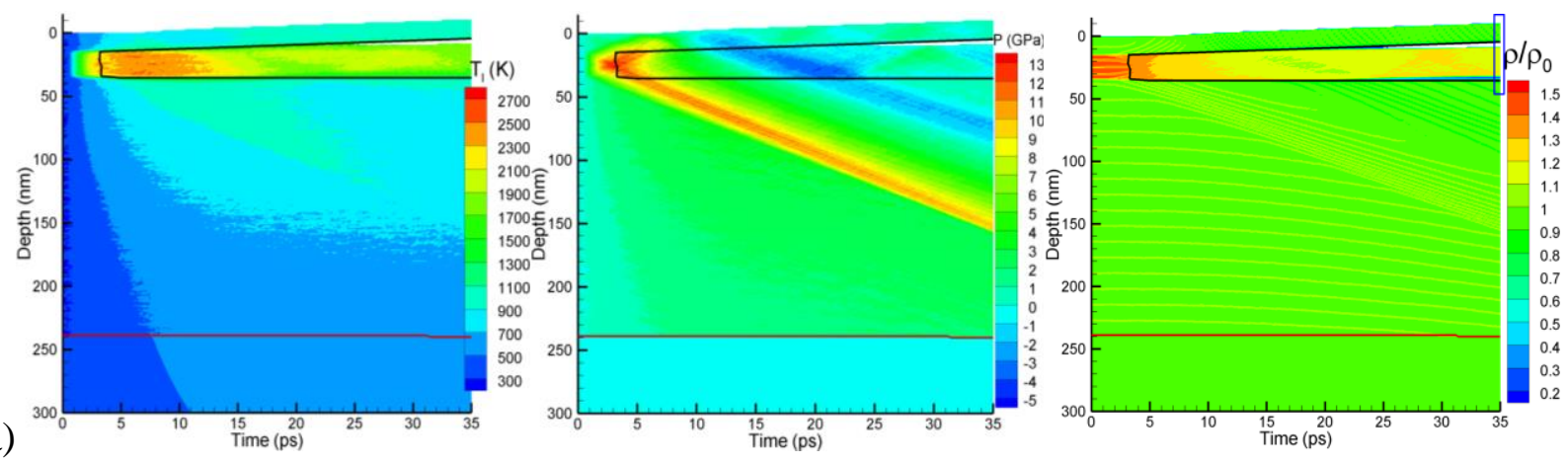

(b)

\section{$35 \mathrm{ps}$ Ag film}

Figure 4.6: The results of a simulation of 200 fs laser irradiation of a $15 \mathrm{~nm} \mathrm{Ag} \mathrm{film} \mathrm{-} 20 \mathrm{~nm} \mathrm{Cu}$ film - Ag (001) substrate system at a fluence of $0.06 \mathrm{~J} / \mathrm{cm}^{2}$. (a) Contour plots of the lattice temperature, pressure, and density as a function of time and depth, with black lines separating the melted regions from the crystalline bulk of the target and red lines separating the MD and continuum parts of the combined TTM-MD model. (b) A snapshot taken for the region marked by the blue rectangle in the density contour plot at a time of $35 \mathrm{ps}$. Spallation at the $\mathrm{Cu}-\mathrm{Ag}$ interface takes place with only intermediate $\mathrm{Cu}$ film melted.

\subsubsection{Threshold fluence for the complete melting of Ag film-Cu film}

Four representative simulations with different fluences, $\mathrm{F}=0.04 \mathrm{~J} / \mathrm{cm}^{2}, 0.05 \mathrm{~J} / \mathrm{cm}^{2}, 0.055$ $\mathrm{J} / \mathrm{cm}^{2}$, and $0.06 \mathrm{~J} / \mathrm{cm}^{2}$ have been performed on a $5 \mathrm{~nm} \mathrm{Ag} \mathrm{film}-5 \mathrm{~nm} \mathrm{Cu}$ film $-\mathrm{Ag}(001)$ substrate system. The evolution of temperature, pressure and density in these simulations is shown in Fig. 4.7. At the low fluence $\mathrm{F}=0.04 \mathrm{~J} / \mathrm{cm}^{2}$, only the intermediate thin $\mathrm{Cu}$ film is melted. At the high fluence $\mathrm{F}=0.06 \mathrm{~J} / \mathrm{cm}^{2}$, the top 2 films are spallated from the $\mathrm{Ag}$ substrate. At the intermediate fluences of $0.05 \mathrm{~J} / \mathrm{cm}^{2}$ and $0.055 \mathrm{~J} / \mathrm{cm}^{2}$, the top two films are completely melted. In the following subsections, we focus on the simulation performed at $\mathrm{F}=0.05 \mathrm{~J} / \mathrm{cm}^{2}$ to study the resolidification, $\mathrm{BCC} \mathrm{Cu}$ growth and its stability. 
(a)
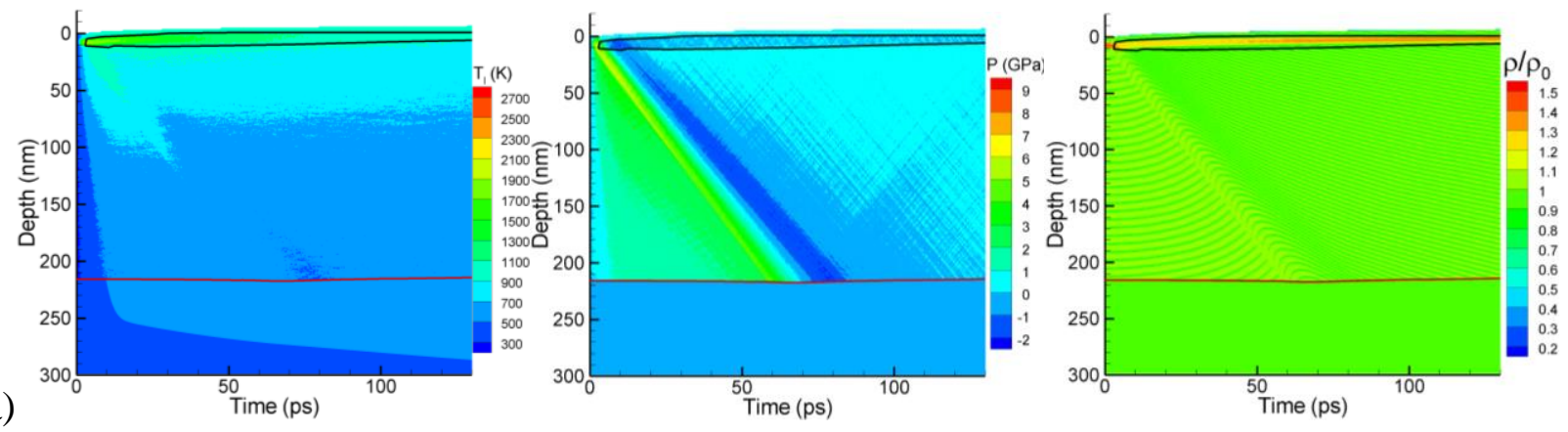

(b)
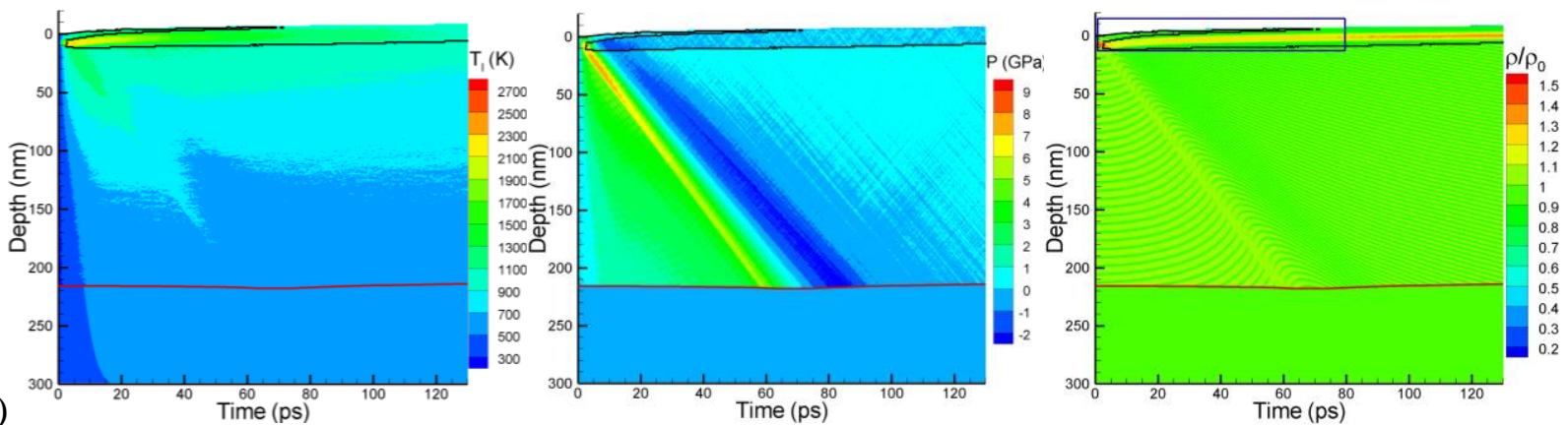

(c)
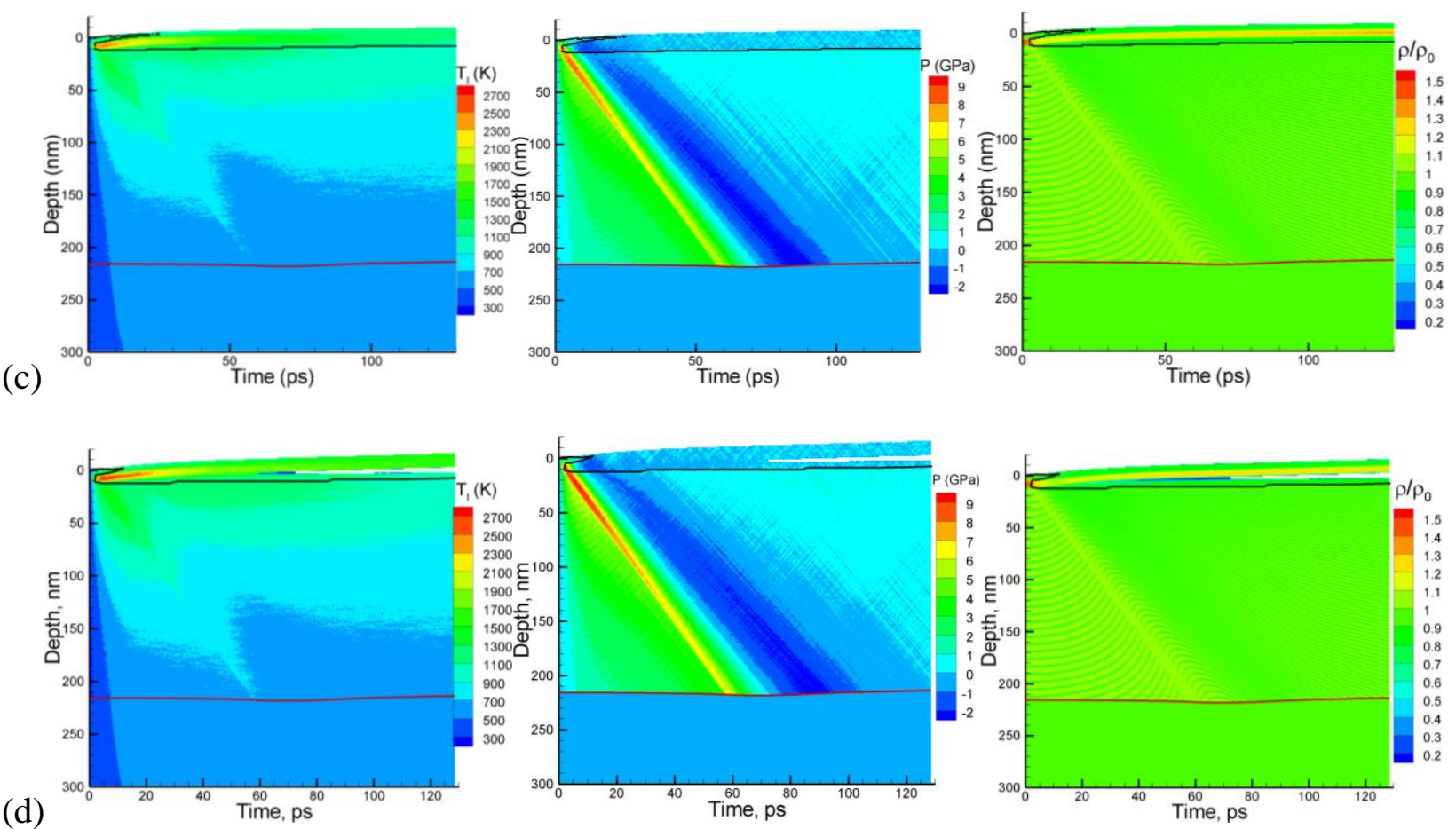

Figure 4.7: Contour plots of the lattice temperature, pressure and density as a function of time and depth for simulations of $5 \mathrm{~nm} \mathrm{Ag} \mathrm{film}-5 \mathrm{~nm} \mathrm{Cu}$ film - Ag (001) substrate systems irradiated with $200 \mathrm{fs}$ laser pulses at absorbed fluences of (a) $0.04 \mathrm{~J} / \mathrm{cm}^{2}$, (b) $0.05 \mathrm{~J} / \mathrm{cm}^{2}$, (c) $0.055 \mathrm{~J} / \mathrm{cm}^{2}$ and (d) $0.06 \mathrm{~J} / \mathrm{cm}^{2}$. Laser pulse is directed along the $\mathrm{Y}$-axis, from the top of the 
contour plots. The black lines separate the melted regions from the crystalline bulk of the target. Red lines separate the MD and continuum parts of the combined TTM-MD model. The two films are spallated at the laser fluence $0.06 \mathrm{~J} / \mathrm{cm}^{2}$ as seen in $(\mathrm{d})$.

\subsubsection{Melting of $\mathrm{Ag}$ and $\mathrm{Cu}$ film}

At the laser fluence of $0.05 \mathrm{~J} / \mathrm{cm}^{2}$, the preferential heating (due to stronger electronphonon coupling of $\mathrm{Cu}$ compared to $\mathrm{Ag}$ ) leads to the rapid melting localized in the intermediate $\mathrm{Cu}$ film, as can be seen from the contour plots in Fig. 4.7c. Then the slow heterogeneous melting is initiated and the whole top $\mathrm{Ag}$ film is melted by the time of $80 \mathrm{ps}$. The melting process after laser irradiation is illustrated in Fig. 4.8.

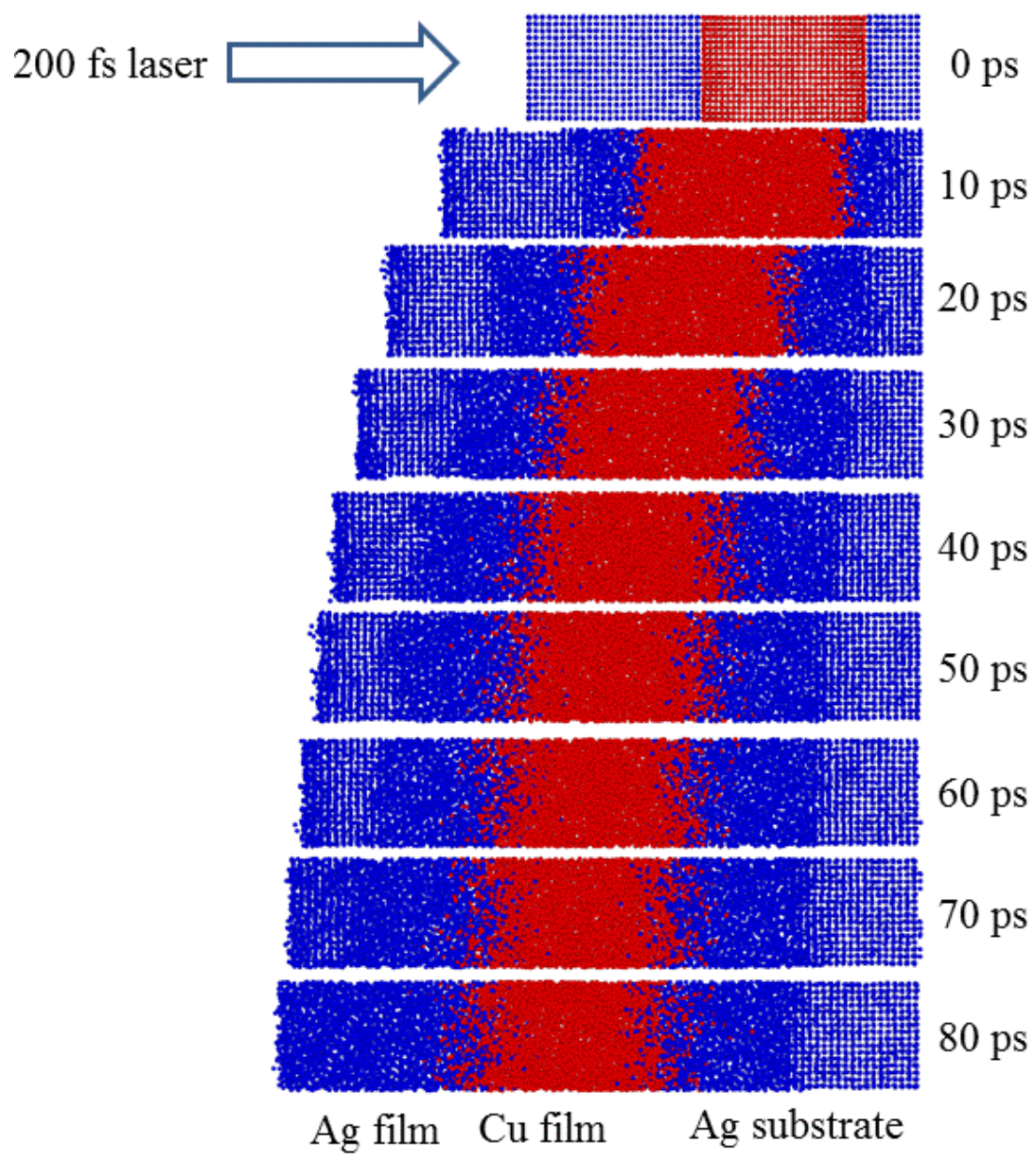

Figure 4.8: Snapshots taken for the region and the time interval marked in Fig. $4.3 \mathrm{c}$ by the blue rectangle. Atoms are colored according to their type. 


\subsubsection{Resolidification: epitaxial growth of Cu on Ag (001) and subsequent growth of Ag on Cu}

For the laser irradiation at $\mathrm{F}=0.05 \mathrm{~J} / \mathrm{cm}^{2}$, the resolidification process is illustrated in Fig. 4.9. A noticeable advancement of the resolidification front from the Ag substrate is observed from $\sim 100 \mathrm{ps}$ after laser irradiation. The velocity of the resolidification front increases with increasing undercooling initially, and then decreases as the resolidification front meets the first $\mathrm{Cu}-\mathrm{Ag}$ mixing region. This slow resolidification can be explained by slower solidification kinetics in binary systems and, more importantly, by the depression of the melting temperature (liquidus line) with respect to the pure $\mathrm{Ag}$ and $\mathrm{Cu}$. After crossing the mixing region, the resolidification front accelerates and propagates through the whole intermediate $\mathrm{Cu}$ film with the velocity of $\sim 130 \mathrm{~m} / \mathrm{s}$. Then the resolidification front slows down again at the second $\mathrm{Cu}-\mathrm{Ag}$ mixing region, and accelerates after this mixing region is crossed. From Fig. 4.9b, we can clearly see the epitaxial growth of $\mathrm{Cu}$ on $\mathrm{Ag}$ (001) and subsequent growth of $\mathrm{Ag}$ on top of $\mathrm{Cu}$ during this heterogeneous resolidification process. The structural analysis in Fig. 4.9c has verified our expectation that the epitaxial growth of $\mathrm{Cu}$ on $\mathrm{Ag}(001)$ leads to the formation of the $\mathrm{BCC} \mathrm{Cu}$ structure and the subsequent epitaxial growth of $\mathrm{Ag}$ on the $\mathrm{BCC} \mathrm{Cu}(001)$ leads to the formation of FCC Ag structure. 

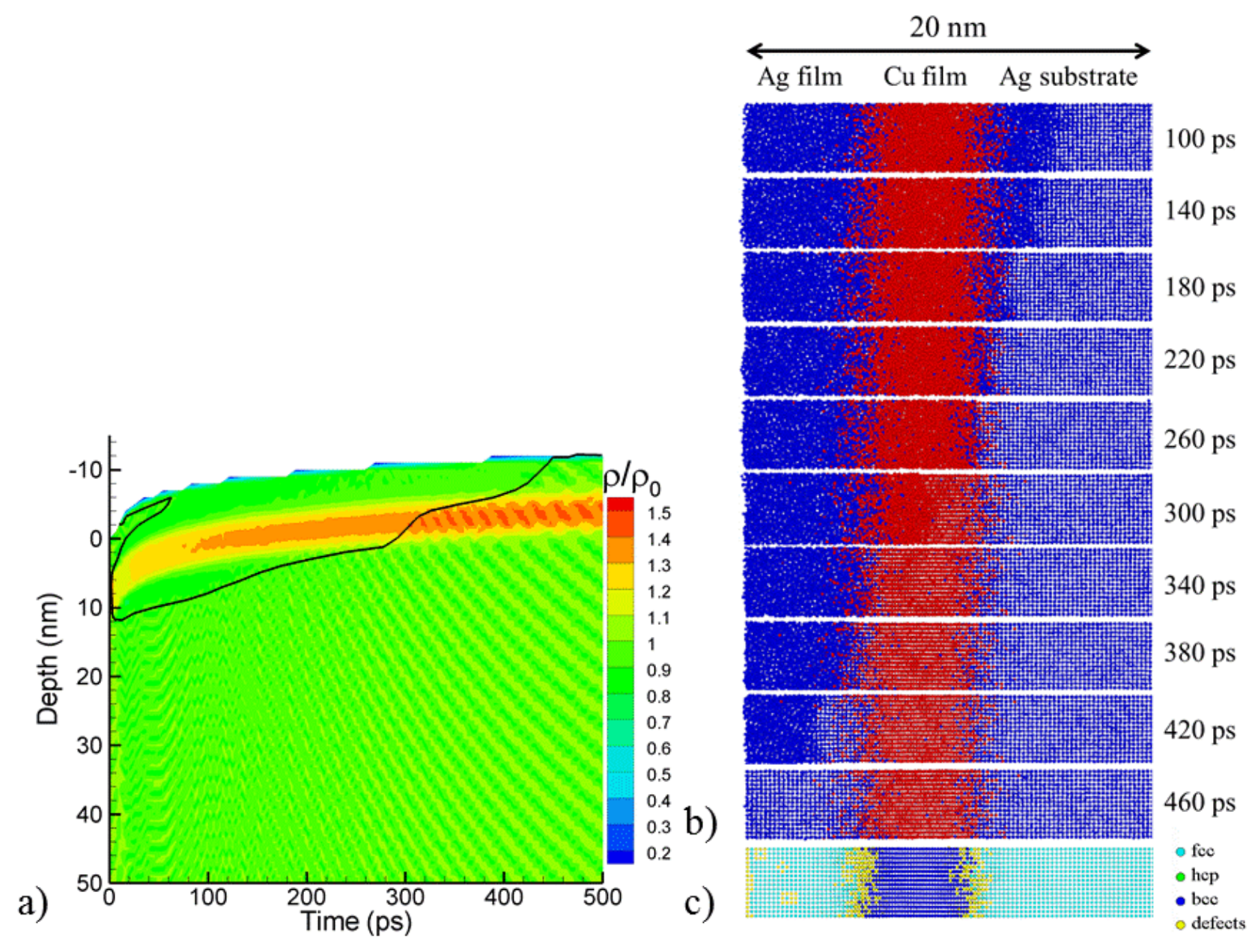

Figure 4.9: (a) Contour plot of density showing the resolidification process after $200 \mathrm{fs}$ laser irradiation of $5 \mathrm{~nm} \mathrm{Ag} \mathrm{film}-5 \mathrm{~nm} \mathrm{Cu}$ film - Ag (001) substrate target at a fluence of $0.05 \mathrm{~J} / \mathrm{cm}^{2}$. The resolidification process can be clearly seen by the slopes of the black lines, which separate the melted regions from the crystalline bulk of the target. (b) Snapshots taken for the top surface region $(\sim 20 \mathrm{~nm})$ in the time interval from $100 \mathrm{ps}$ to $460 \mathrm{ps}$. Atoms are colored by their type, with red color used for $\mathrm{Cu}$ and blue color for Ag. (c) Snapshot at $460 \mathrm{ps}$ with atoms colored by their local structural environment. To reduce the thermal noise, the system is quenched for 0.2 ps before the structural analysis.

\subsubsection{Stability of the intermediate $\mathrm{BCC} \mathrm{Cu}$ film}

After the formation of the BCC Cu layer sandwiched between FCC Ag (001) substrate and the top $\mathrm{Ag}(001)$ layer, the system is relaxed for $\sim 5 \mathrm{~ns}$, during which it is naturally cooled down due to the electron heat conduction that is taken into account in our TTM-MD model. 
Even though the temperature of the system drops down to $\sim 420 \mathrm{~K}$ at the end of the simulation, the intermediate BCC Cu layer stays stable as seen by comparing Figs. $4.10 \mathrm{~b}$ and $4.10 \mathrm{c}$.

To check the stability of the BCC structure upon further cooling more quickly, we performed a separate MD simulation, in which the final atomic configuration obtained in the TTM-MD simulation is cooled at a faster rate of $0.5 \mathrm{~K} / \mathrm{ps}$. The system is cooled down to $300 \mathrm{~K}$ and then kept at $300 \mathrm{~K}$ for additional $200 \mathrm{ps}$. The snapshot of atomic structure of the surface region, shown in Fig. 4.10d, clearly indicates that the intermediate BCC Cu layer stays stable at $300 \mathrm{~K}$. To further test the stability of the $\mathrm{BCC} \mathrm{Cu}$, the system is then cooled down from $300 \mathrm{~K}$ to $0 \mathrm{~K}$. The snapshot shown in Fig. 4.10e indicates that the intermediate $\mathrm{BCC} \mathrm{Cu}$ layer is stable even at $0 \mathrm{~K}$.

(a)

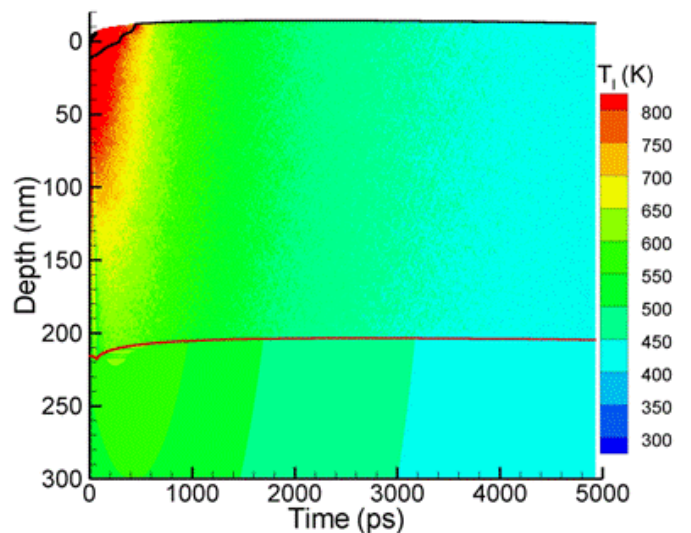

(b)

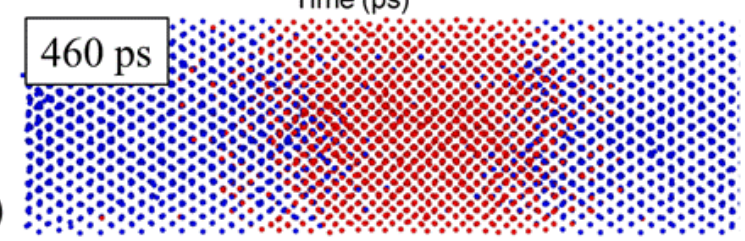

(c)

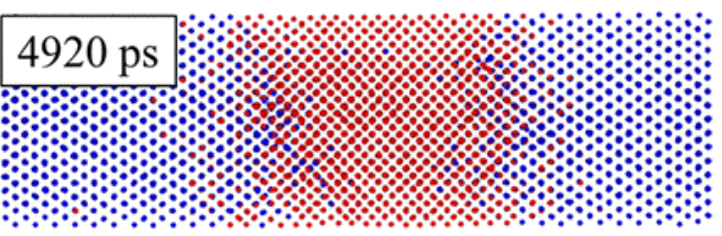

(d)

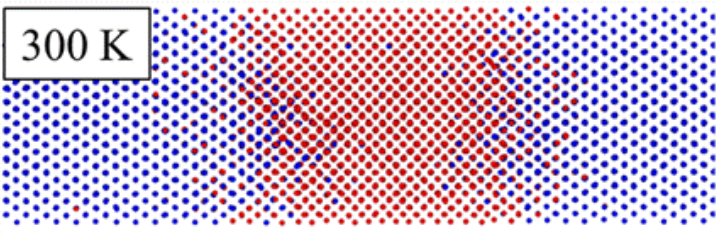

(e)

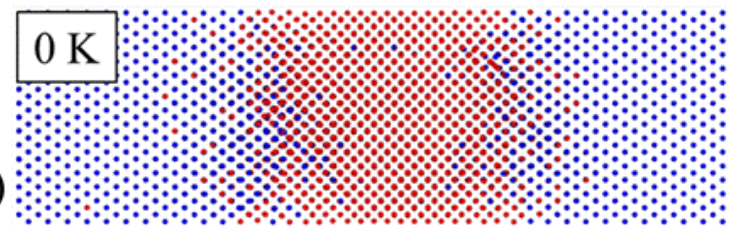

Figure 4.10: (a) Contour plot describing lattice temperature evolution during TTM-MD simulation of $200 \mathrm{fs}$ laser irradiation of $5 \mathrm{~nm} \mathrm{Ag} \mathrm{film}-5 \mathrm{~nm} \mathrm{Cu}$ film $-\mathrm{Ag}$ (001) substrate target at a fluence of $0.05 \mathrm{~J} / \mathrm{cm}^{2}$. The cooling of the system down to $\sim 400 \mathrm{~K}$ during the $5 \mathrm{~ns}$ of the simulation can be seen from the contour plot. (b)-(d) Snapshots of the top surface region ( 15 $\mathrm{nm}$ ) viewed from [110] direction of the original FCC structure. Atoms are colored by their type, with red color used for $\mathrm{Cu}$ and blue color used for Ag. (b) is taken at the time $460 \mathrm{ps}$ after the laser irradiation. (c) is taken at $4920 \mathrm{ps}$ after the laser irradiation, when the system has uniform 
temperature of $\sim 420 \mathrm{~K}$. (d) is taken after the system is equilibrated at $300 \mathrm{~K}$ for $200 \mathrm{ps}$, and (e) is taken after the system is cooled down to $0 \mathrm{~K}$.

\subsection{Concluding remarks}

Serveral conclusions can be made based on the computational results reported in this section.

First, we have further verified the possibility of $\mathrm{BCC} \mathrm{Cu}$ formation through the epitaxial growth on top of $\mathrm{Ag}(001)$ during the melting-resolidification cycle induced by short pulse laser irradiation. The formation of $\mathrm{BCC} \mathrm{Cu}$ can be related to the good match between the lattice parameter of the $\mathrm{BCC} \mathrm{Cu}$ structure and the first-neighbor distance in the FCC Ag structure, as well as the relatively small energy difference between the FCC and BCC $\mathrm{Cu}$ structures (the difference in the cohesive energies predicted with the FBD EAM potential is $22 \mathrm{meV} / \mathrm{atom}$ ). Second, while the presence of Ag (001) substrate on one side of BCC Cu film can help stabilize the $\mathrm{BCC} \mathrm{Cu}$, it cannot hold the $\mathrm{BCC}$ structure of the $(\sim 20 \mathrm{~nm}) \mathrm{Cu}$ film down to the room temperature. The BCC Cu structure transforms into a mixture of FCC and HCP regions when it cools below $\sim 450 \mathrm{~K}$. This observation may be related to the experimental failure to observe the $\mathrm{BCC} \mathrm{Cu}$ beyond 10 monolayers. Most importantly, we find that a relatively thick, 5 nm layer of $\mathrm{BCC} \mathrm{Cu}$ can be stabilized down to the room temperature (and even down to $0 \mathrm{~K}$ ) when it is sandwiched by the FCC Ag (001) on both sides.

It is important to note that the choice of the FCC crystal structure oriented along [001] direction for the $(\mathrm{Ag} / \mathrm{Cu})$ films in our computational setup is made just for the computational convenience and does not affect the results of the simulations. Since the films are completely melted shortly after the laser pulse, the memory of their initial orientation and even the crystal structure is lost. In the other words, experimental verification of the computational predictions does not require the $\mathrm{Ag} / \mathrm{Cu}$ films be oriented along the [001] direction in the initial sample.

These conclusions of the study are not restricted in the field of short pulse laser processing. They also provide an idea for possible formation of "stable" BCC Cu by atomic deposition. As reported [70,71], BCC Cu can be deposited on FCC Ag (001) substrate up to 10 monolayers, and the surface buckles and transforms to FCC structure upon further deposition. Based on our simulations, a new deposition process can be proposed. First, $\sim 9$ monolayers of (BCC) $\mathrm{Cu}$ on $\mathrm{Ag}$ (001) substrate are deposited. Then several Ag monolayers are deposited on 
top of the $\mathrm{BCC} \mathrm{Cu}$, and the deposition steps of $\mathrm{Cu}$ and $\mathrm{Ag}$ are repeated. By doing this, it is theoretically possible to generate multilayered structure containing metastable $\mathrm{BCC} \mathrm{Cu}$ layers stabilized by coherent (001) interfaces with FCC Ag. 


\section{Femtosecond laser ablation of $\mathrm{Ag}(001)$ - experiments and small-scale simulations}

\subsection{Introduction}

Femtosecond laser ablation (material removal) is widely used in high-precision micromachining of solid surfaces. The ablation mechanism is a topic of ongoing discussion [43] and its better understanding is required to further optimize the experimental parameters in current applications, and to invent new applications. Experimentally, our collaborators from the group of Professor Peter Balling at the Aarhus University in Denmark have recently observed the surprising surface swelling on single-crystal surface (Al (111) [19] and Ag (001) [20]) during short-pulse ( $100 \mathrm{fs})$ laser irradiation at the laser fluence close to the ablation threshold. This observation suggests the important role of the strong mechanical effects during laser excitation, i.e., photomechanical effects. The continuum-level simulations based on TTM have been performed to study the short-pulse laser ablation [95]. The biggest limitation of TTM simulations, however, is that it can only treat the ablation as a completely thermal process (no stress involved). In other words, evaporation is assumed to be the dominant mechanism of laser ablation, which contradicts the experimental observations. In particular, the TTM simulations cannot capture the surface swelling behavior and fully explain the ablation mechanism. On the other hand, the combined TTM-MD model naturally accounts for both the thermal effects (heating and cooling, vaporization and explosive boiling) and the photomechanical effects (deformation in response to thermoelastic stresses, spallation), and is suitable for the investigation of the laser ablation mechanism. In this section, the key results of single-shot laser ablation experiments performed by Martin Snogdahl Christensen working in the group of Professor Peter Balling at the Aarhus University in Denmark are firstly reviewed. Most of these results have not been published yet and are made available to us by Prof. Balling. Then results of a series of small-scale TTM-MD simulation results are presented. The results of the simulations are compared with experimental observations and the limitations of the small-scale TTM-MD simulations are discussed. 


\subsection{Single-shot femtosecond laser ablation experiments}

The ablation experiment is performed in an ultra-high vacuum (UHV, $\sim 10^{-9}$ Torr) chamber. The experimental setup is shown in Fig. 5.1. Prior to the laser irradiation, the singlecrystal (Al (111) and $\mathrm{Ag}(001))$ surfaces are cleaned by a sputter gun with $1 \mathrm{keV} \mathrm{Ar}^{+}$ions $\left(\sim 3 \times 10^{-5}\right.$ Torr $)$ for $\sim 1$ hour, and subsequently annealed at $\sim 940 \mathrm{~K}$ for $\mathrm{Ag}(001)$ and $\sim 700 \mathrm{~K}$ for $\mathrm{Al}$ (111) for another $\sim 1$ hour to eliminate the possible defects introduced during the sputtering process. To ensure a smooth suface, the sputtering/annealing cycles are repeated 3-5 times. The laser has a pulse duration of $\sim 100 \mathrm{fs}$ and a wavelength of $\sim 800 \mathrm{~nm}$. The spatial profile is Gaussian and, the fluence given in this subsection refers to the peak incident fluence in the center of the laser spot if not otherwise indicated. The adsorbed fluence can be easily calculated from the Fresnel reflectivity of 0.969 at $800 \mathrm{~nm}$.

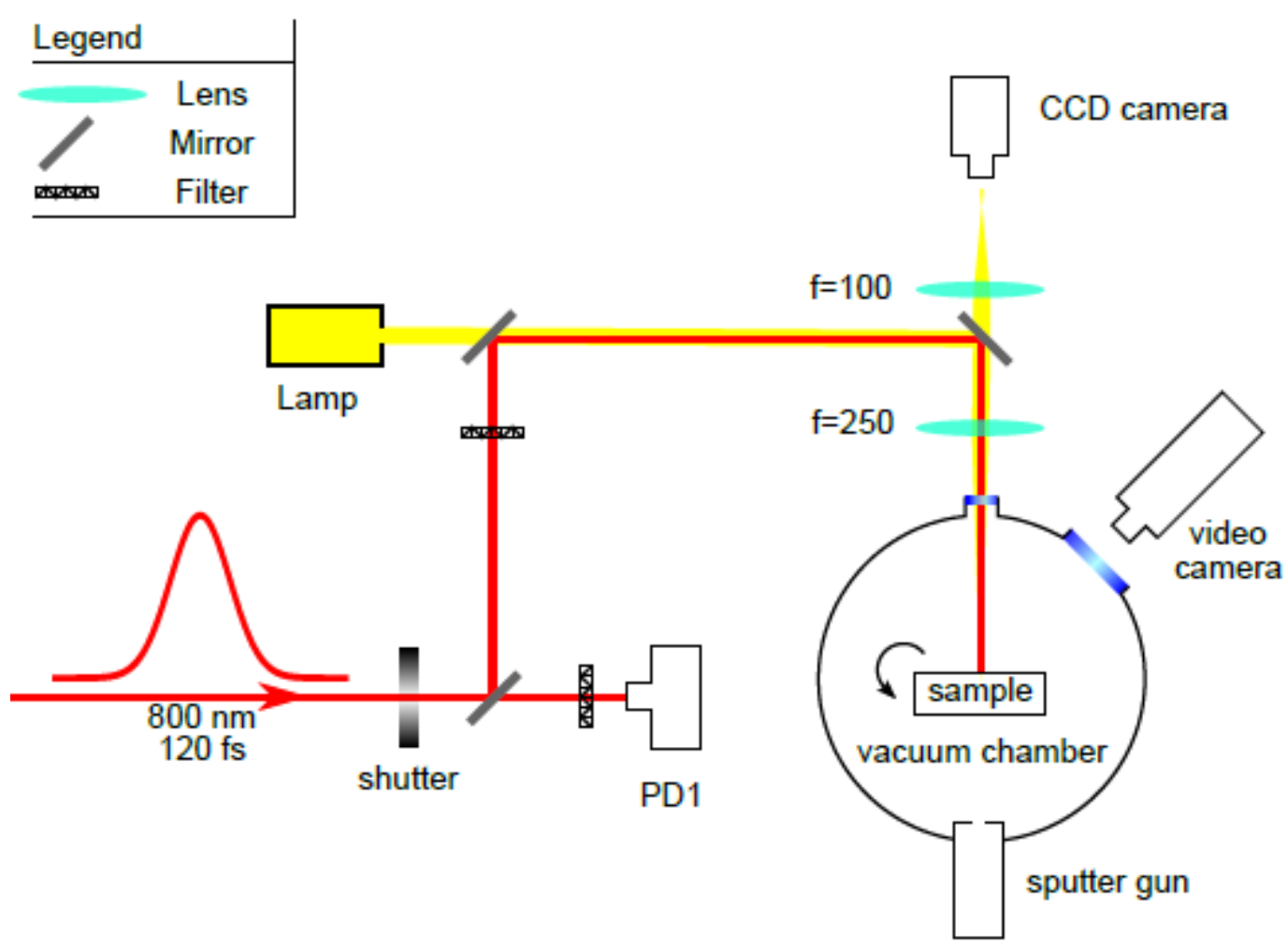

Figure 5.1: Setup for UHV laser ablation experiments. The laser beam is focused onto the sample using a $\mathrm{f}=250 \mathrm{~mm}$ achromatic lens. This figure is adopted from Ref. [20].

Several SEM images of ablation spots at different fluences around and above the ablation threshold are shown in Fig. 5.2 for the Ag (001) surface, which shows the bubble-like structures 
increasing in size with laser fluence. Additional information related to the ablation mechanism can be gained by studying the topography of the ablation spots from the atomic-force microscopy (AFM) images. Fig. 5.3 presents the line outs of AFM images of the ablation spots on Ag (001) surfaces generated at different fluences. From Fig. 5.3, it is clearly seen that the bumps (100s to 1000s nanometers) are formed on the surface, i.e., surface swelling occurs. Only at the highest fluence, the material is removed down to the levels below the initial surface. In addition, the threshold fluences of swelling and material removal obtained from experiments are listed in table 1.

\begin{tabular}{|ccllcc} 
EXP & $\begin{array}{c}\text { Incident } \\
\text { fluence } \\
\left(\mathrm{J} / \mathrm{cm}^{2}\right)\end{array}$ & $\begin{array}{c}\text { Adsorbed } \\
\text { fluence } \\
\left(\mathrm{J} / \mathrm{cm}^{2}\right)\end{array}$ & TTM-MID & $\begin{array}{c}\text { Incident } \\
\text { fluence } \\
\left(\mathrm{J} / \mathrm{cm}^{2}\right)\end{array}$ & $\begin{array}{c}\text { Adsorbed } \\
\text { fluence } \\
\left(\mathrm{J} / \mathrm{cm}^{2}\right)\end{array}$ \\
\hline Swelling & $2.5 \pm 0.1$ & $0.078 \pm 0.003$ & Melting & 1.94 & 0.06 \\
\hline Removal & $3.17 \pm 0.06$ & $0.098 \pm 0.002$ & $\begin{array}{l}\text { Removal/S } \\
\text { welling }\end{array}$ & $2.58-2.90$ & $0.08-0.09$
\end{tabular}

Table 1: Threshold fluences of melting, swelling and material removal from experiments and small-scale TTM-MD simulations. Both incident and adsorbed fluences are given, which can be converted using the Fresnel reflectivity, $R=0.969$, for the short pulse laser (with wavelength 800 $\mathrm{nm}$ ) irradiation on $\mathrm{Ag}$.

In fact, this surprising surface swelling behavior is not specific to the short pulse laser excitation of the Ag (001) system. Similar behavior has also been observed on Al (111) system by Prof. Peter Balling et al. [19]: "at low fluence, swelling exceeding 100 nanometers dominates, while at higher fluences, a hole is gradually formed in the swollen material, which eventually reaches below the original surface level." The AFM line outs of the ablated spots on Al (111) surface can be seen in Fig. 5.4, showing the evolution from bump to a deep hole for increasing fluences. The surface swelling can be thought of "the first step in the ablation of metals by ultrashort laser pulses". As discussed in [19], the surface swelling may be related to the front-side photomechanical spallation [43,96], and can be described as "frustrated ablation", where the material seems to be frozen in porous foamy structure with voids of different sizes. In the following subsection, the TTM-MD simulations are performed to explore the hidden mechanism of the surface swelling and laser ablation. 

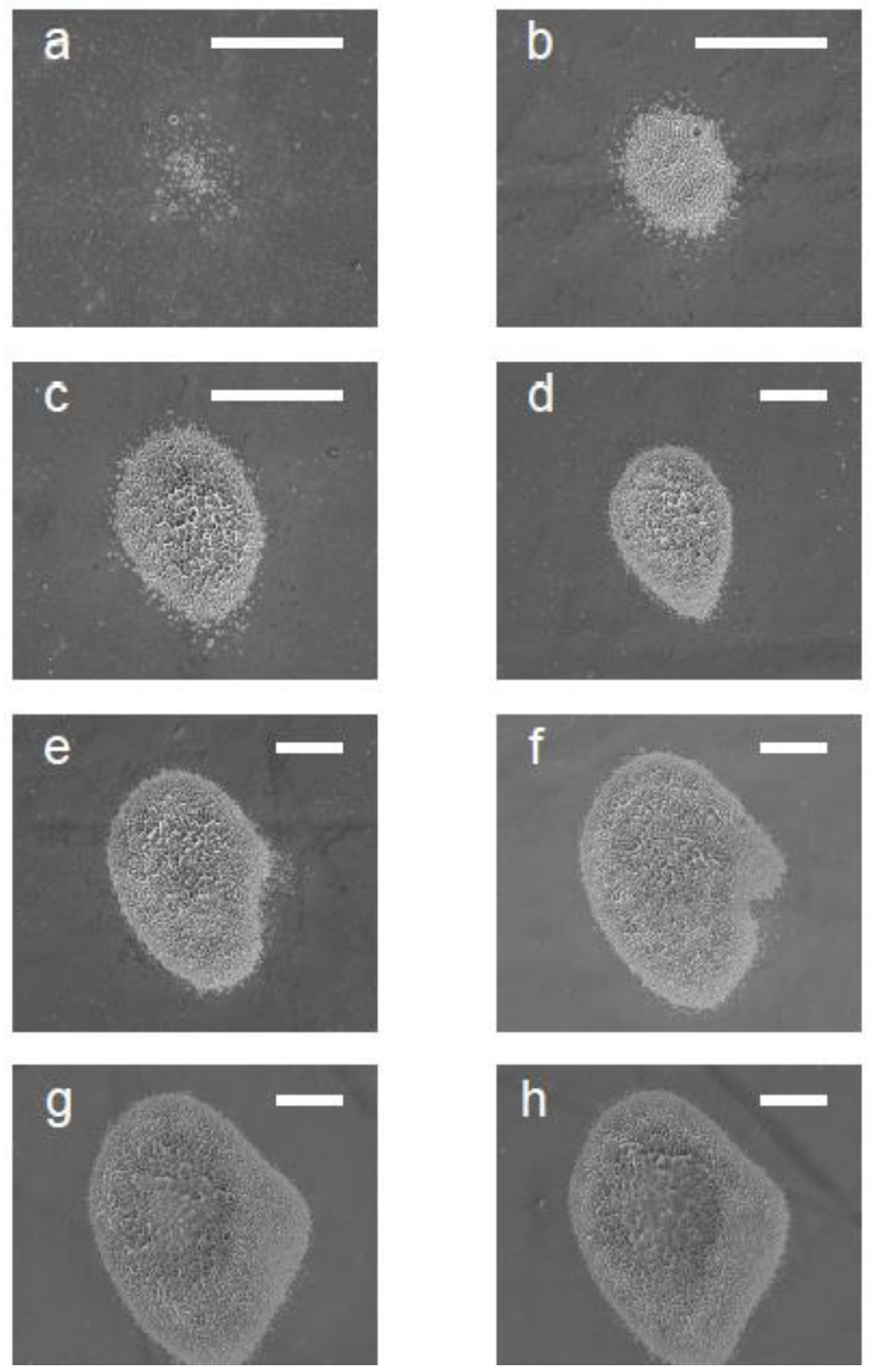

Figure 5.2: Series of SEM images of the ablation spots on $A g(001)$ surfaces irradiated by $100 \mathrm{fs}$ laser pulses at incident fluences from $2.45 \mathrm{~J} / \mathrm{cm}^{2}$ to $42.6 \mathrm{~J} / \mathrm{cm}^{2}$ : (a) $2.45 \pm 0.06 \mathrm{~J} / \mathrm{cm}^{2}$, (b) $3.90 \pm 0.07 \mathrm{~J} / \mathrm{cm}^{2}$, (c) $5.70 \pm 0.08 \mathrm{~J} / \mathrm{cm}^{2}$, (d) $9.40 \pm 0.10 \mathrm{~J} / \mathrm{cm}^{2}$, (e) $15.10 \pm 0.20 \mathrm{~J} / \mathrm{cm}^{2}$, (f) $24.1 \pm 0.3$ $\mathrm{J} / \mathrm{cm}^{2}$, (g) $34.9 \pm 0.4 \mathrm{~J} / \mathrm{cm}^{2}$ and (h) $42.6 \pm 0.5 \mathrm{~J} / \mathrm{cm}^{2}$. The white scale bar in each image corresponds to $20 \mu \mathrm{m}$. This figure is adopted from Ref. [20]. 
(a)
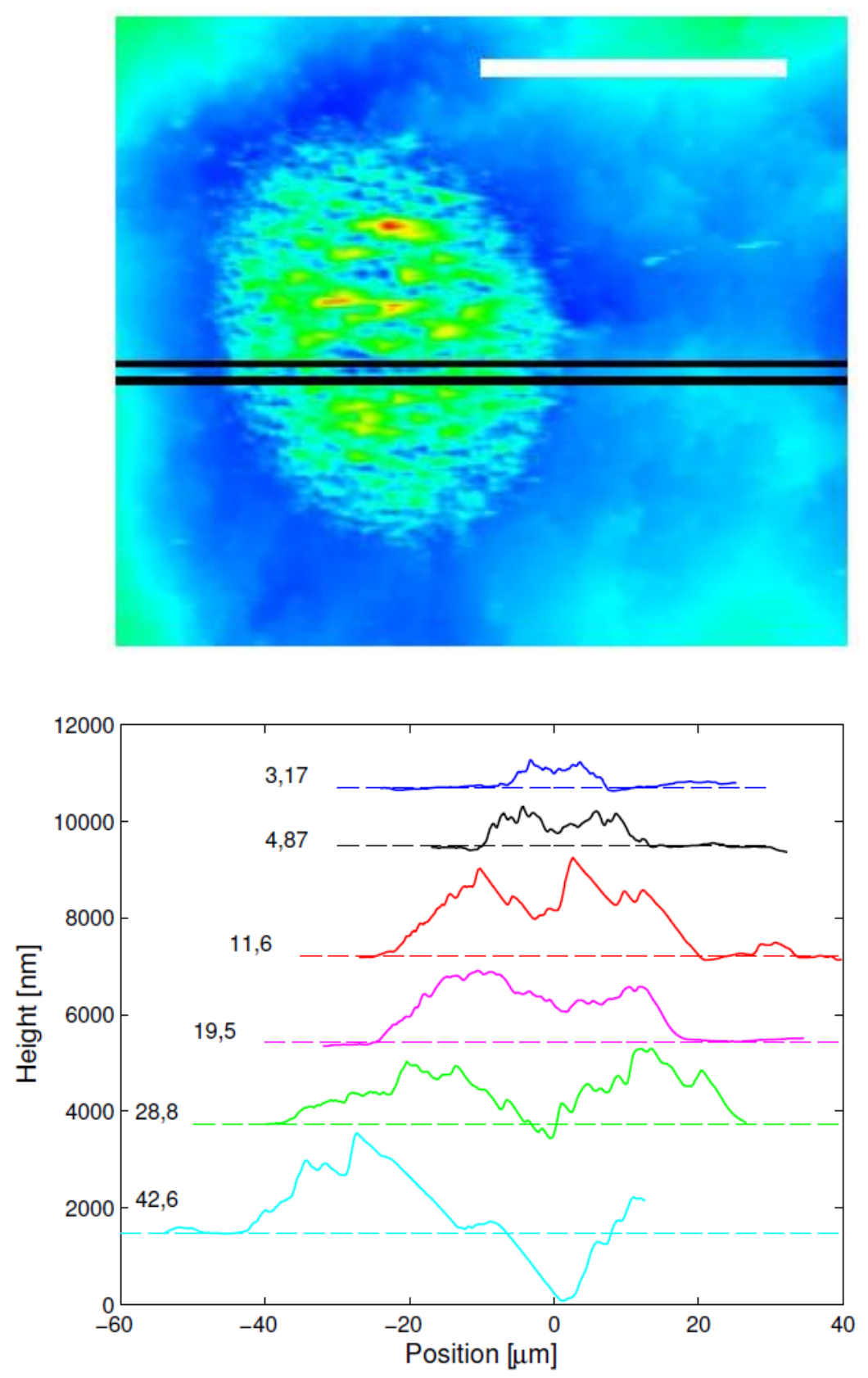

(b)

Figure 5.3: (a) An example of AFM surface scan of an ablation spot at incident laser fluence of $4.87 \pm 0.08 \mathrm{~J} / \mathrm{cm}^{2}$. The black horizontal lines mark the area from which the average line out is computed as shown in (b). (b) AFM line outs of several ablation spots on Ag (001) surface generated by irradiation at incident fluences from $3.17 \mathrm{~J} / \mathrm{cm}^{2}$ (absorbed fluence of $0.098 \mathrm{~J} / \mathrm{cm}^{2}$ ) to $42.6 \mathrm{~J} / \mathrm{cm}^{2}$ (adsorbed fluence of $1.321 \mathrm{~J} / \mathrm{cm}^{2}$ ). For clarity, the curves are offset vertically. The bumps are formed on the surfaces and the material is removed down to the levels below the initial surface level at the highest fluence. This figure is adopted from Ref. [20]. 


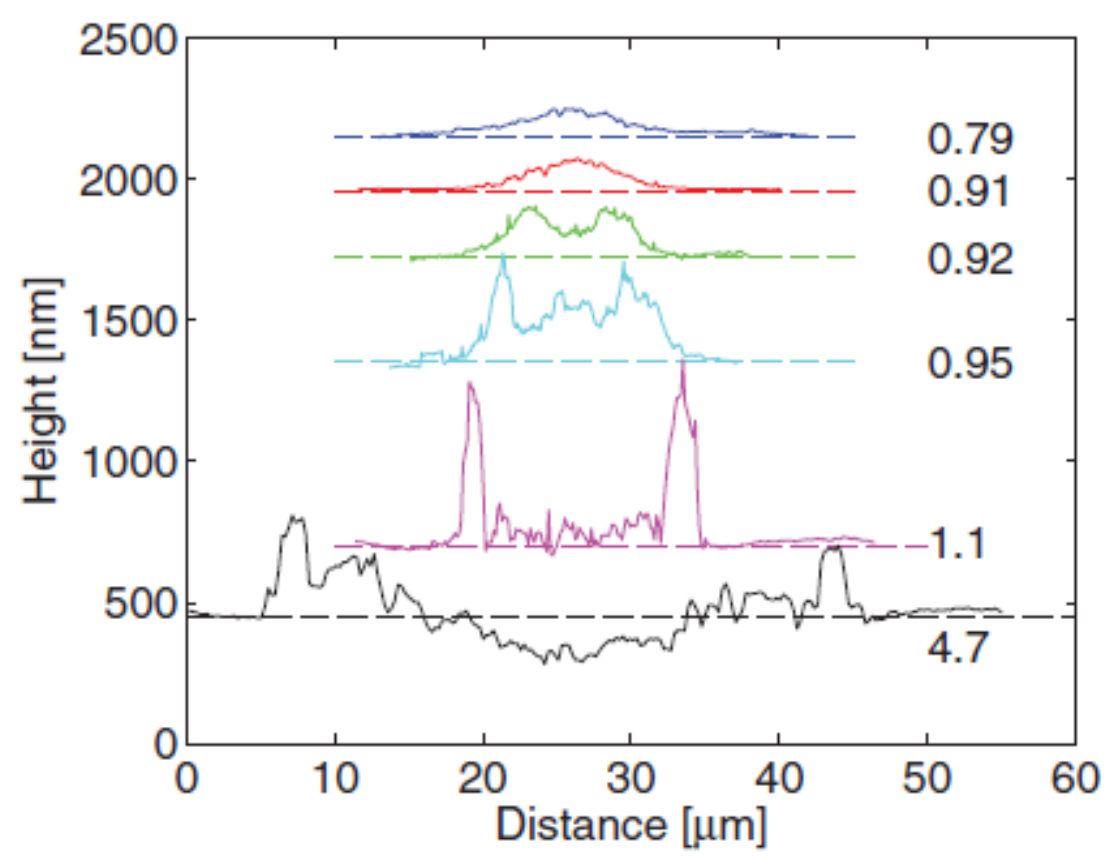

Figure 5.4: AFM line outs of the ablation spots observed on $\mathrm{Al}$ (111) surface irradiated by 100 fs laser pulses at different incident fluences ranging from $0.79 \mathrm{~J} / \mathrm{cm}^{2}$ to $4.7 \mathrm{~J} / \mathrm{cm}^{2}$. For clarity, the curves are offset vertically. This figure is adopted from Ref. [19].

\subsection{TTM-MD simulations of single-shot laser (100 fs) irradiation of Ag (001) target}

The computational setup is similar to the one used in the simulations of $\mathrm{Ag}-\mathrm{Cu}$ layered targets (sections 3 and 4), as shown in Fig. 5.5. Before running large-scale simulation (discussed in the next section, section 6), we perform small-scale simulations to get a big picture of short pulse laser interaction with with $\mathrm{Ag}$ (001) for a broad range of laser fluences. In addition, to get a feeling of the lateral size dependence of the simulation results, two series of simulations are performed for systems of two different sizes: one with $L_{x}=L_{y}=2.47 \mathrm{~nm}$, the other with $L_{x}=4.94 \mathrm{~nm}$ and $L_{y}=9.88 \mathrm{~nm}$. In this subsection, the fluence given is the adsorbed fluence

\section{if not otherwise indicated.}




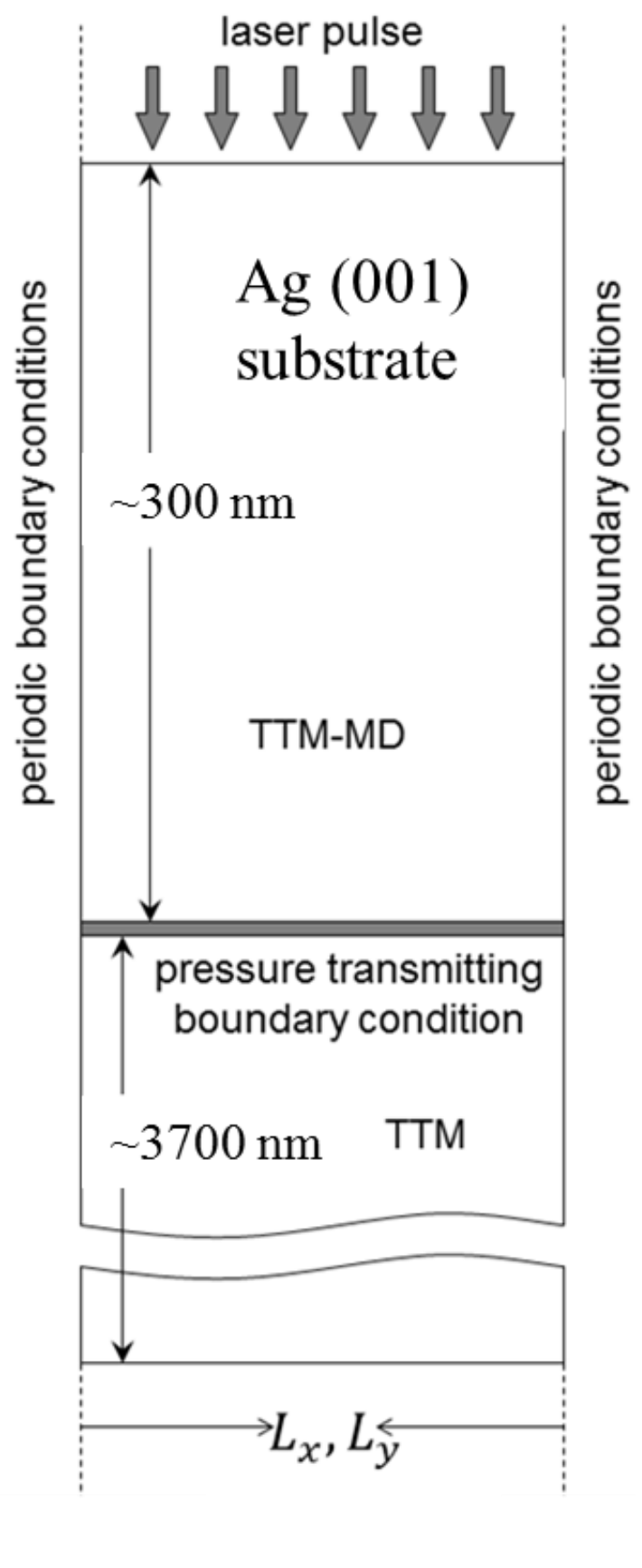

Figure 5.5: Schematic sketch of the simulation setup. TTM-MD model is applied in the surface region of the target, down to $\sim 300 \mathrm{~nm}$, whereas the electron heat conduction in the deeper part of the substrate, down to $4 \mu \mathrm{m}$, is simulated with the conventional TTM.

\subsubsection{Threshold fluence for laser melting}

Fig. 5.6 presents the temperature and pressure evolution during and after laser irradiation obtained in simulations performed at fluences $F=0.05 \mathrm{~J} / \mathrm{cm}^{2}$ and $\mathrm{F}=0.07 \mathrm{~J} / \mathrm{cm}^{2}$. At the fluence 
$\mathrm{F}=0.05 \mathrm{~J} / \mathrm{cm}^{2}$, the maximum lattice temperature just reaches the melting temperature and no melting occurs in the target. At the fluence $\mathrm{F}=0.07 \mathrm{~J} / \mathrm{cm}^{2}$, several layers are melted down to a depth of $\sim 20 \mathrm{~nm}$. Comparing the two temperature contour plots, we expect the threshold fluence for surface melting to be $\sim 0.06 \mathrm{~J} / \mathrm{cm}^{2}$.

(a)
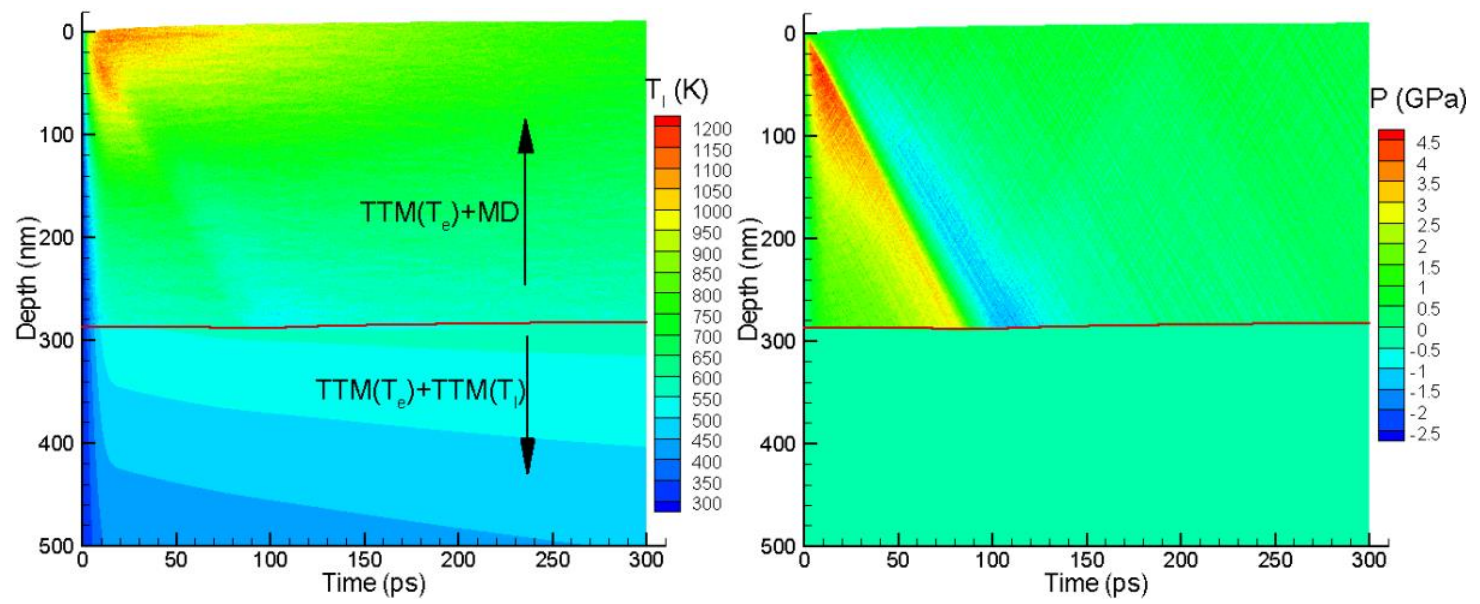

(b)
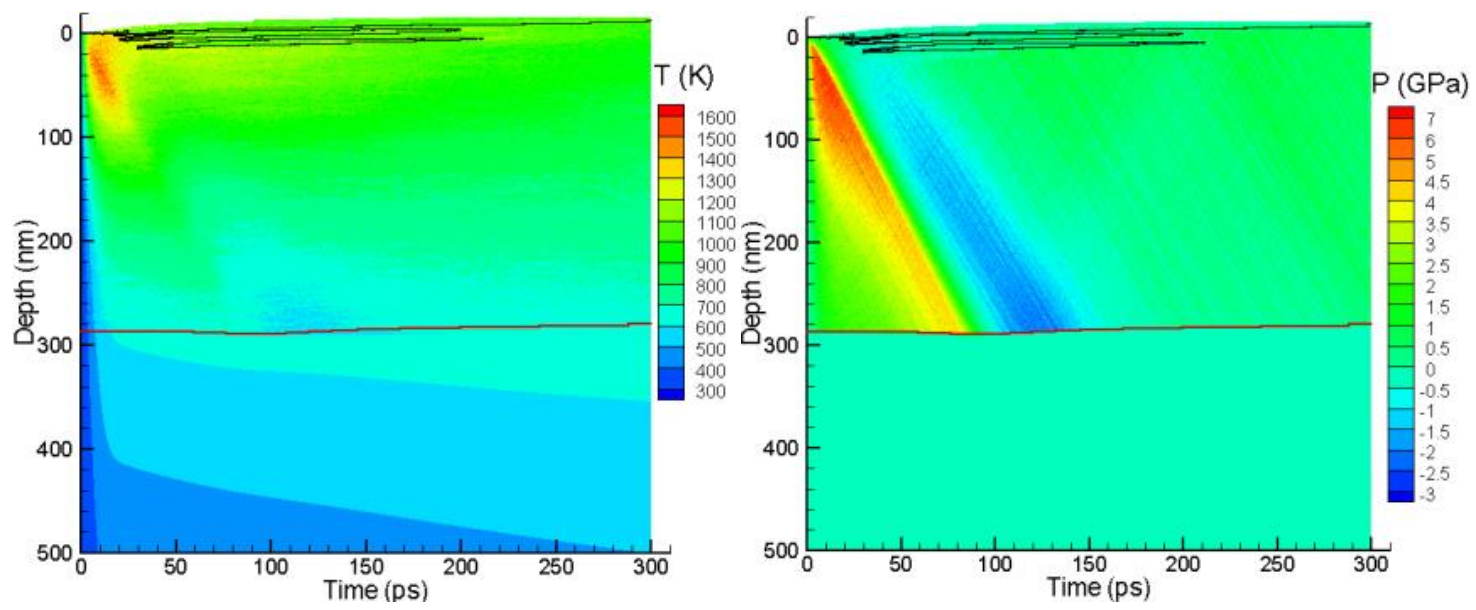

Figure 5.6: Contour plots of the lattice temperatureand pressure as a function of time and depth obtained in simulations of $100 \mathrm{fs}$ pulse laser irradiation of $\mathrm{Ag}(001)$ at fluences of (a) $0.05 \mathrm{~J} / \mathrm{cm}^{2}$, and (b) $0.07 \mathrm{~J} / \mathrm{cm}^{2}$. Laser pulse is directed along the Y-axis, from the top of the contour plots. The black lines separate the melted regions from the crystalline bulk of the target. Red lines separate the MD and continuum parts of the combined TTM-MD model. The simulated system has the lateral size $L_{x}=L_{y}=2.47 \mathrm{~nm}$. 


\subsubsection{Threshold fluence for spallation or void formation}

Fig. 5.7 presents temperature, pressure and density evolution during and after laser irradiation obtained in simulations performed at fluences $F=0.08 \mathrm{~J} / \mathrm{cm}^{2}$ and $F=0.09 \mathrm{~J} / \mathrm{cm}^{2}$. At the fluence $\mathrm{F}=0.08 \mathrm{~J} / \mathrm{cm}^{2}, a \sim 50 \mathrm{~nm}$ surface layer is melted after laser irradiation. At the higher fluence $\mathrm{F}=0.09 \mathrm{~J} / \mathrm{cm}^{2}$, the voids are formed in the liquid and then two liquid layers are ejected or "spallated" from the target. The observed void formation is realistic, but the ejection is probably related to the small lateral size, maximum $9.88 \mathrm{~nm}$, of the simulation system. Due to the small lateral size, the percolation of the voids, once they are formed and grow, can easily lead to the separation and ejection of the liquid layers. This is illustrated in Fig. 5.8: as voids are nucleated, grow and coalescence, they can easily reach the lateral size of the computational cell, maximum $9.88 \mathrm{~nm}$, and then the ejection happens. As soon as the ejection happens, the stress is then quickly relaxed which help the collapse of the other voids as seen in Fig. 5.8. In this sense, it is more appropriate to say that the threshold fluence of void formation is greater than 0.08 $\mathrm{J} / \mathrm{cm}^{2}$ but less than $0.09 \mathrm{~J} / \mathrm{cm}^{2}$ based on these simulations. This void formation is of photomechanical nature, as discussed below.

(a)
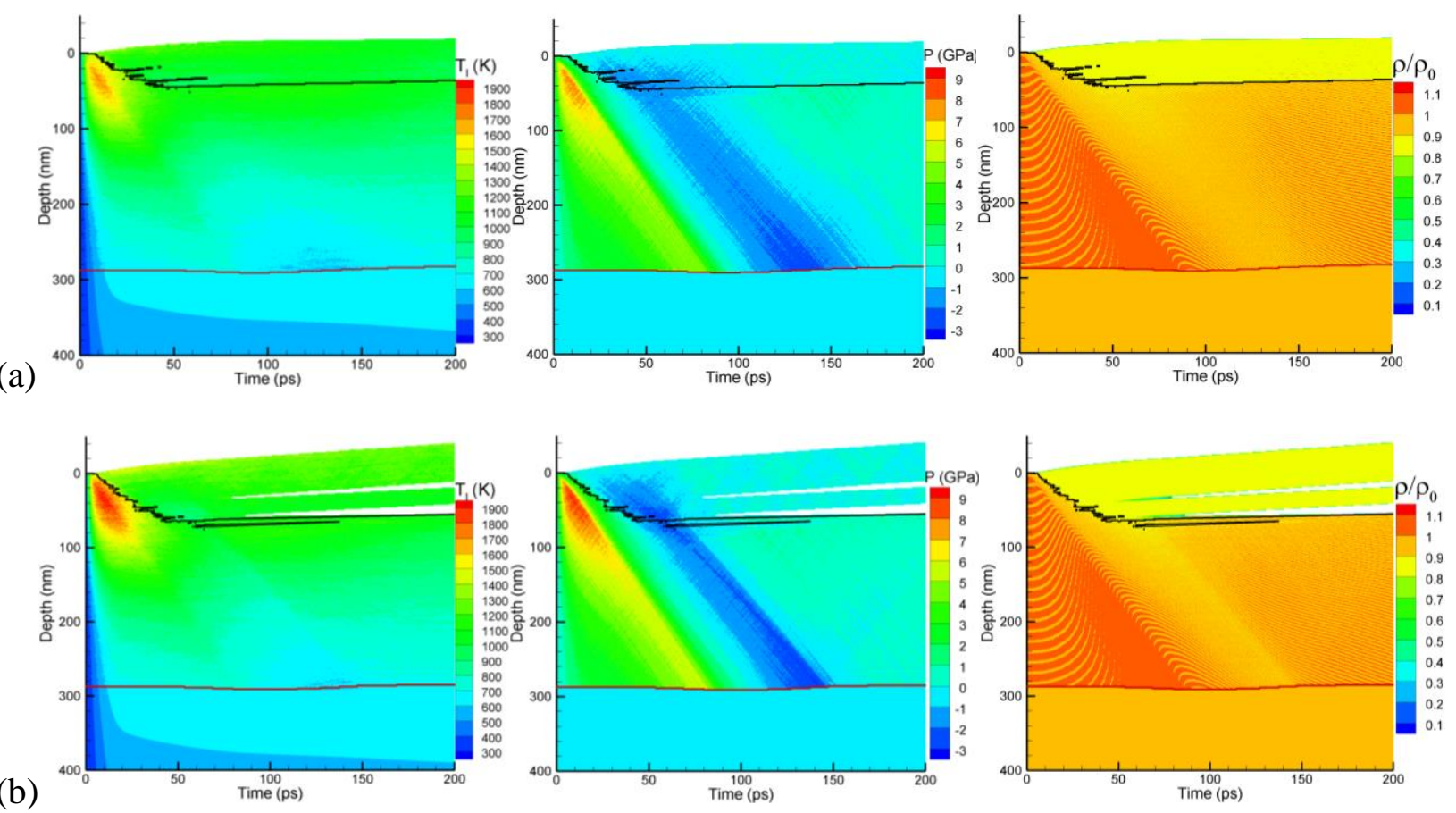

Figure 5.7: Contour plots of the lattice temperature, pressure and density (normalized to the density of $\mathrm{Ag}$ at $300 \mathrm{~K}$ before the irradiation) as a function of time and depth for simulations of $100 \mathrm{fs}$ pulse laser irradiation of $\mathrm{Ag}$ (001) at absorbed fluences of (a) $0.08 \mathrm{~J} / \mathrm{cm}^{2}$, and (b) 0.09 
$\mathrm{J} / \mathrm{cm}^{2}$. Laser pulse is directed along the Y-axis, from the top of the contour plots. The black lines separate the melted regions from the crystalline bulk of the target. Red lines separate the MD and continuum parts of the combined TTM-MD model. The simulated system has the lateral size $L_{x}=L_{y}=2.47 \mathrm{~nm}$.
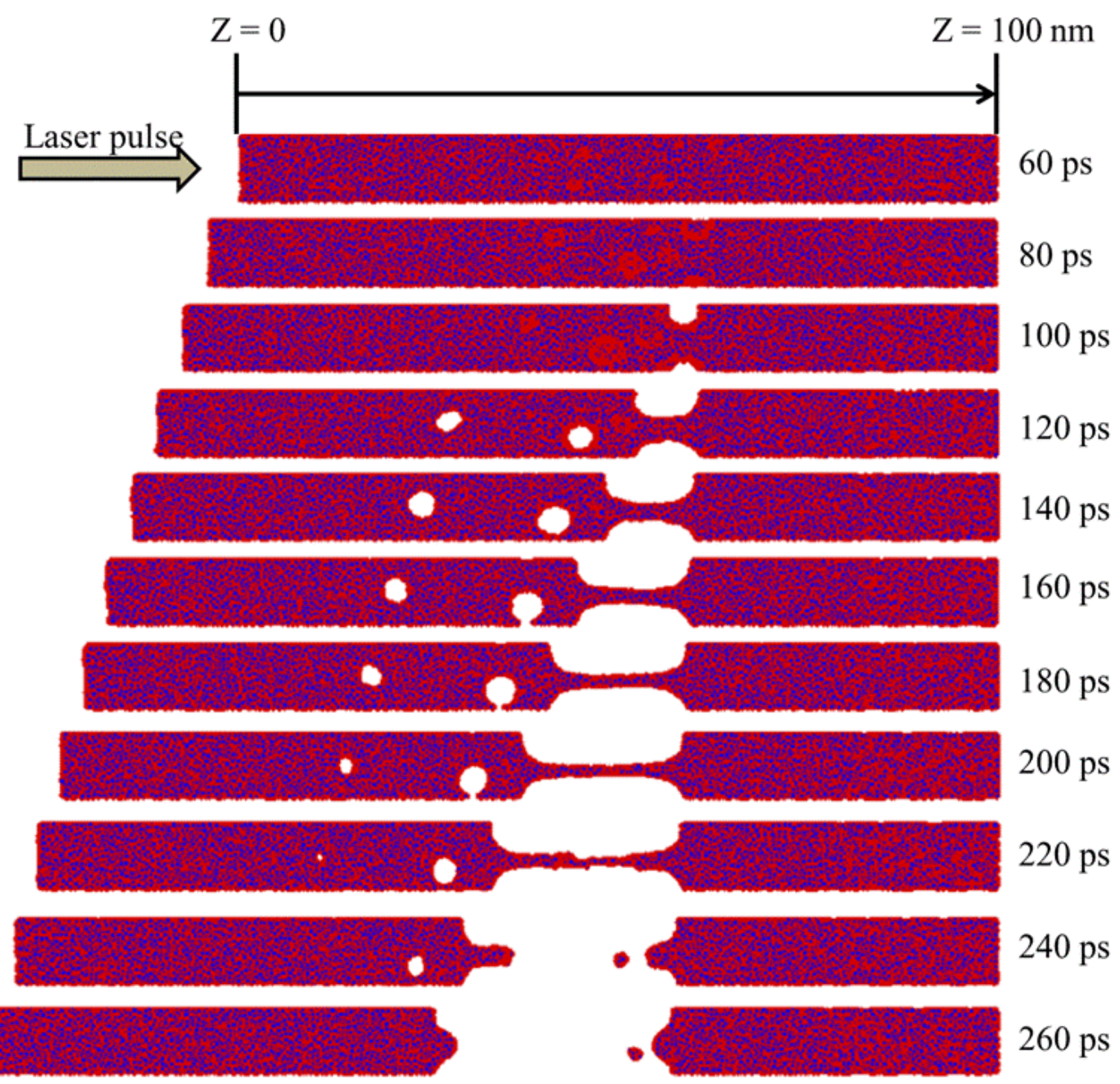

Figure 5.8: Snapshots of the top $100 \mathrm{~nm}$ of the $\mathrm{Ag}(001)$ target irradiated by a $100 \mathrm{fs}$ laser pulse at a fluence of $0.10 \mathrm{~J} / \mathrm{cm}^{2}$. The snapshots show the process of voids formation and subsequent 
ejection of a melted surface layer. from 60 ps to 260 ps at 20 ps time intervals. The computation cell has the lateral size $L_{x}=4.94 \mathrm{~nm}$ and $L_{y}=9.88 \mathrm{~nm}$.

\subsubsection{Photomechanical effects}

The fast energy deposition in short pulse laser irradiation not only leads to a sharp temperature rise in the adsorption region of the target but may also result in the generation of compressive stresses $[43,96]$. The build-up of compressive stresses is defined by the relationship between the characteristic time of laser heating, $\tau_{h}$, and the time required for the mechanical relaxation of the heated volume, $\tau_{r}$. When the time of laser heating is less than the required mechanical relaxation time, i.e., $\tau_{h} \leq \tau_{r}$, the heating takes place under a nearly constant volume condition, leading to the build-up of compressive stresses, also referred to as the condition of stress confinement [96]. In the case of short pulse irradiation on metals, the lattice heating time is the maximum of the laser pulse duration, $\tau_{p}$, and the characteristic time of electron-phonon coupling, $\tau_{e-p h}$, i.e., $\tau_{h}=\max \left\{\tau_{p}, \tau_{e-p h}\right\}$. On the other hand, the mechanical relaxation time is defined by the size of the laser heating region, $L_{c}$, which has the lower limit defined by the effective laser energy penetration depth (the sum of optical and ballistic penetration depths), and the sound speed of the material, $c$, i.e., $\tau_{r} \sim L_{c} / c$. Thus, the condition for generation of the compressive stresses can be formulated as $\tau_{h}=\max \left\{\tau_{p}, \tau_{e-p h}\right\} \leq \tau_{r} \sim L_{c} / c$. In the case of $100 \mathrm{fs}$ laser irradiation on $\mathrm{Ag}$ (001), the lattice heating time is limited by time of the electron-phonon equilibration, $\sim 10 \mathrm{ps}$. The time required for mechanical relaxation can be estimated as, $\tau_{r} \sim L_{c} / c \sim 68 \mathrm{~nm} / 2.7 \mathrm{~nm} / \mathrm{ps} \sim 25 \mathrm{ps}$. Thus, the condition of stress confinement is satisfied and the compressive stresses build up as the red spot near the surface seen in the pressure contour plots in Figs. 5.6 and 5.7.

The compressive stress relaxes by driving a compressive pressure wave deeper into the bulk of the target, seen as the red stripe in the pressure contour plots of Figs. 5.6 and 5.7, and in the direction to the free surface. The reflection of the compressive pressure wave from the irradiated free surface transforms it to an unloading tensile wave that follows the compressive wave propagating deeper into the target and increasing its strength with depth. The tensile wave can be seen as the blue stripe seen in the pressure contour plots of Figs. 5.6 and 5.7. This 
unloading tensile wave drives the void formation, as clearly seen in Fig. 5.7b, which shows the coincidence of the appearance of the voids (and subsequent spallation) with the arrival of the unloading tensile wave in the melted region. The mechanical stability of the surface region is strongly affected (decreased) by laser heating and melting. Therefore, the region of the void formation is much closer to the surface as compared with the region of maximum tensile stresses. The subsequent separation and ejection of the liquid layers take place by nucleation, growth, and coalescence of multiple voids in the subsurface region of the target. By analogy with the term "spallation" commonly used to describe the dynamic fracture that results from the reflection of a shock wave from a back surface of a sample, the observed laser induced material ejection is called "front-side photomechanical spallation".

\subsubsection{Size dependence of simulation results}

As discussed above, the small lateral size of the computational cell leads to the artificially easy spallation of the liquid layers in the simulations. Some notion of the size dependence of simulation results can be obtained by comparing simulation results obtained with differently sized computational cells: one with $L_{x}=L_{y}=2.47 \mathrm{~nm}$ and the other with $L_{x} \sim 4.94 \mathrm{~nm}$ and $L_{y} \sim 9.88 \mathrm{~nm}$. Fig. 5.9 presents simulation results obtained at the laser fluence $\mathrm{F}=0.10 \mathrm{~J} / \mathrm{cm}^{2}$ with at two systems of different size, but otherwise identical conditions. Both simulations exhibit the void formation due to the action of the tensile pressure wave, as can be seen by comparing the pressure and density contour plots. For the smaller system of $L_{x}=L_{y}=2.47 \mathrm{~nm}$, the two liquid layers are spallated almost immediately after the voids are formed, at $\sim 50$ ps. For the larger system of $L_{x} \sim 4.94 \mathrm{~nm}$ and $L_{y} \sim 9.88 \mathrm{~nm}$, the spallation also happens, but at a later time of $\sim 190$ ps and only one liquid layer is spallated. The delayed spallation is due to the fact that more time is needed for the voids to grow and coalescence to the size of the larger computational cell. These differences of the two simulations demonstrate the artificial effect introduced by the small lateral size of the computational cell used in these simulations. 
(a)
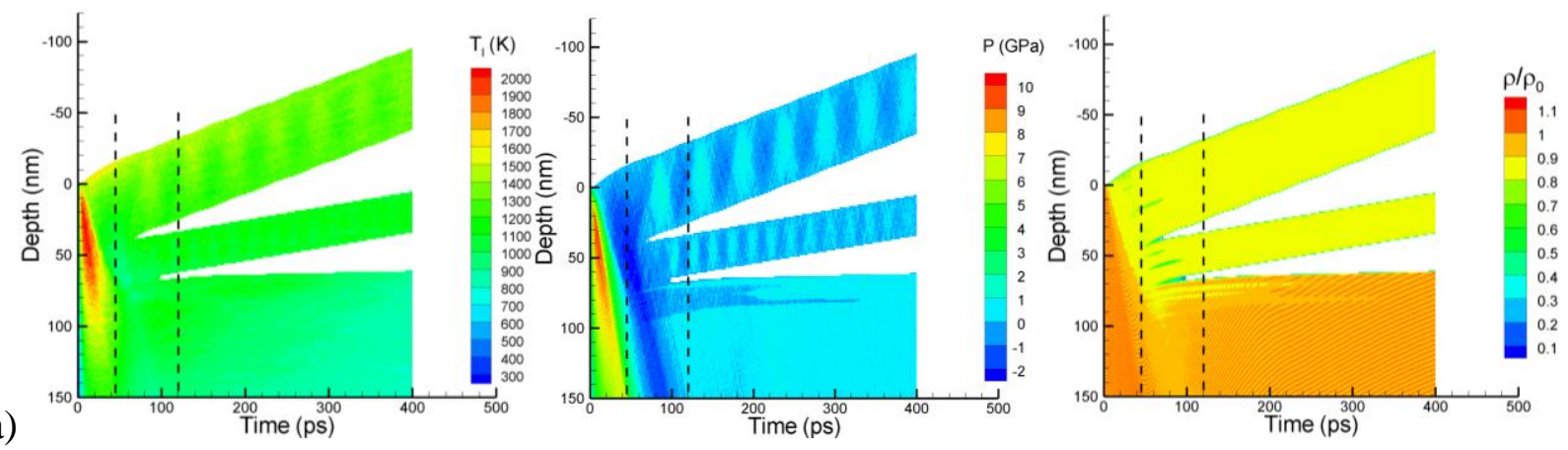

(b)
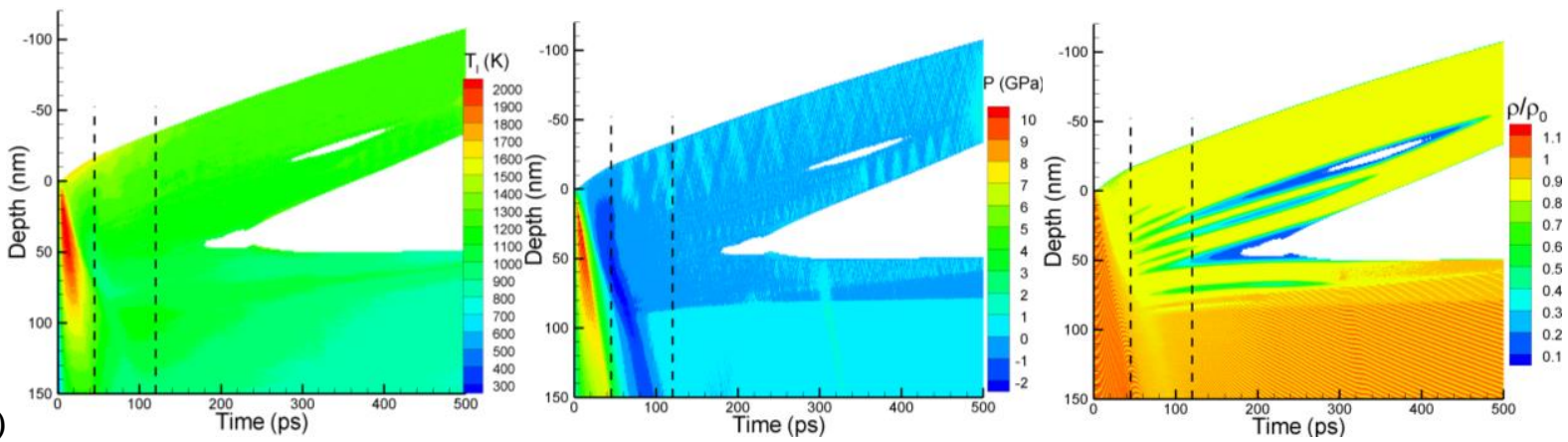

Figure 5.9: Contour plots of the lattice temperature, pressure and density as a function of time and depth for simulations of 100 fs pulse laser irradiation of $\mathrm{Ag}(001)$ at fluences of $0.10 \mathrm{~J} / \mathrm{cm}^{2}$. The simulations are performed for two systems of different lateral size: (a) $L_{x}=L_{y}=2.47 \mathrm{~nm}$ and (b) $L_{x} \sim 4.94 \mathrm{~nm}$ and $L_{y} \sim 9.88 \mathrm{~nm}$.

\subsubsection{Transition from spallation to phase explosion}

As the laser fluence increases from the spallation threshold up to $0.20 \mathrm{~J} / \mathrm{cm}^{2}$, more liquid layers are photomechanically spallated from the bulk target, as can be seen in Fig. 5.10a and 5.10b. Going to even higher fluence, $0.25 \mathrm{~J} / \mathrm{cm}^{2}$, the lattice is heated above $\sim 4000 \mathrm{~K}$ in the surface region and phase explosion is initiated. The phase explosion is commonly discussed as a primary mechanism of short pulse laser ablation of metal targets at high fluence [43,37,97-101], in which the material ejection involves an explosive decomposition of a surface region of the target superheating beyond the limit of thermodynamic stability of the target material (threshold temperature for phase explosion, $\sim 90 \%$ of the critical temperature) into a two-phase mixture of 
liquid and vapor. Following a method applied earlier to a system of Ar atoms [102], the threshold temperature for the onset of phase explosion for FBD EAM Ag material is determined by Eaman Tahir Abdul Karim in constant pressure MD simulations of a slow heating of a metastable liquid, and found to be $\sim 3500 \mathrm{~K}$ at zero pressure, $\sim 4900 \mathrm{~K}$ at the pressure of $0.5 \mathrm{GP}$, and $\sim 6000 \mathrm{~K}$ at the pressure of $1.0 \mathrm{GPa}$. The threshold temperature for phase explosion can be expected to be $\sim 10 \%$ below the critical temperature of the material. The phase explosion temperature of Ag predicted with the FBD EAM potential agrees reasonably with the reported critical temperature that ranges from $4300 \mathrm{~K}$ to $7500 \mathrm{~K}$ with the critical pressure from $0.03 \mathrm{GPa}$ to $0.5 \mathrm{GPa}$ [103]. The initiation of phase explosion does not eliminate the photomechanical spallation, which is still taking place underneath, where the lattice temperature is below the threshold temperature for phase explosion. When the fluence is increased even more, a larger region is heated above phase explosion, and more atoms are ablated by the phase explosion mechanism.

(a)
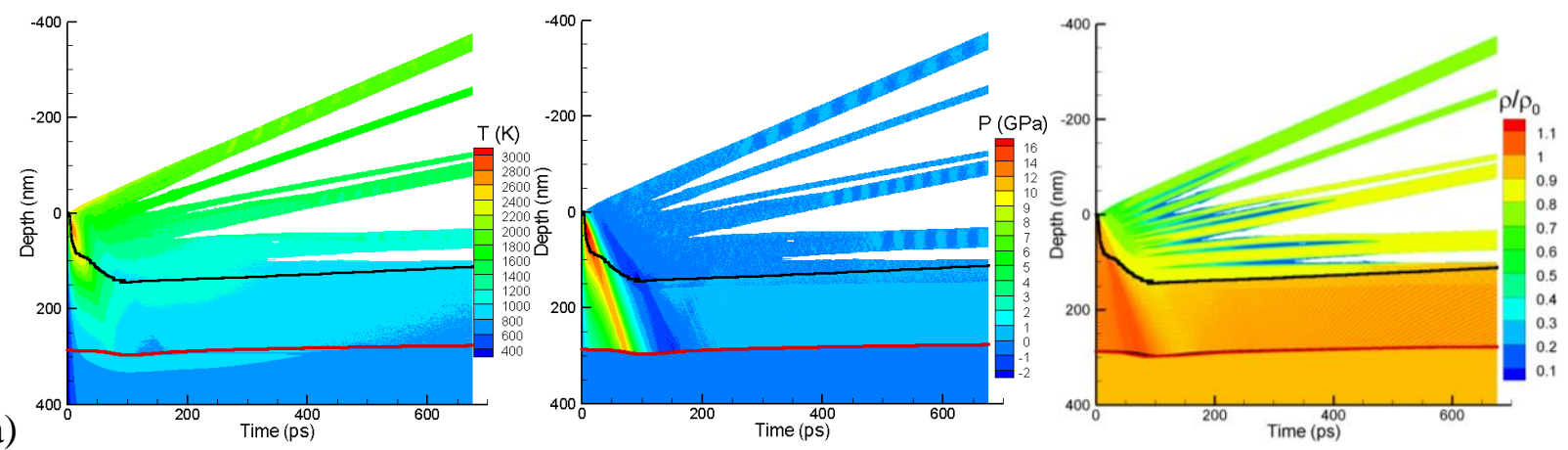

(b)
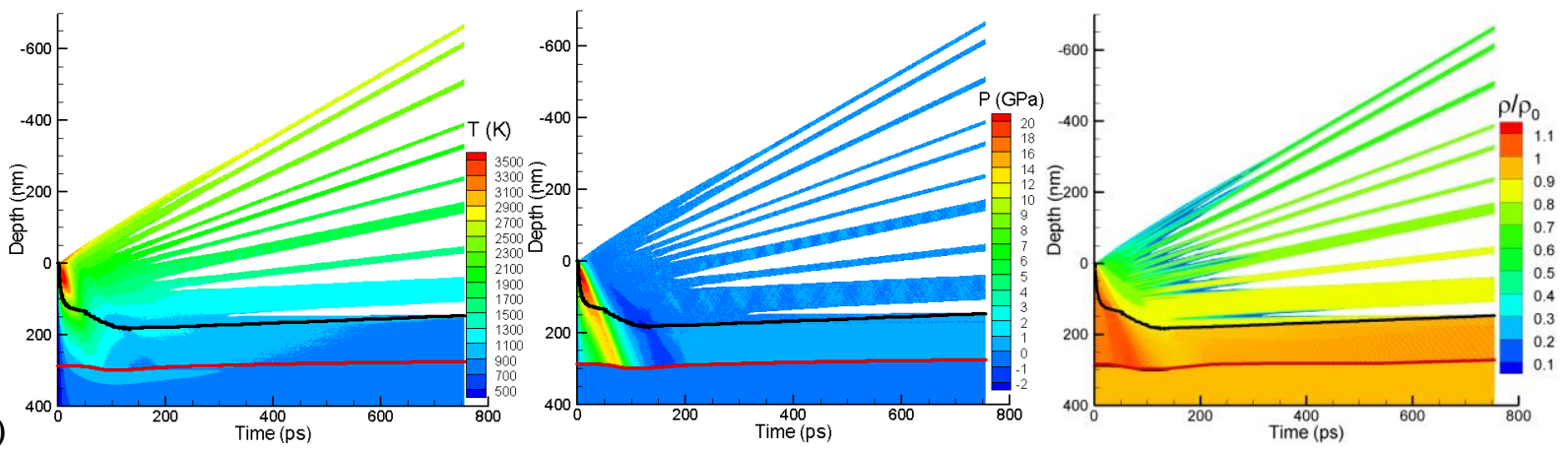
(c)
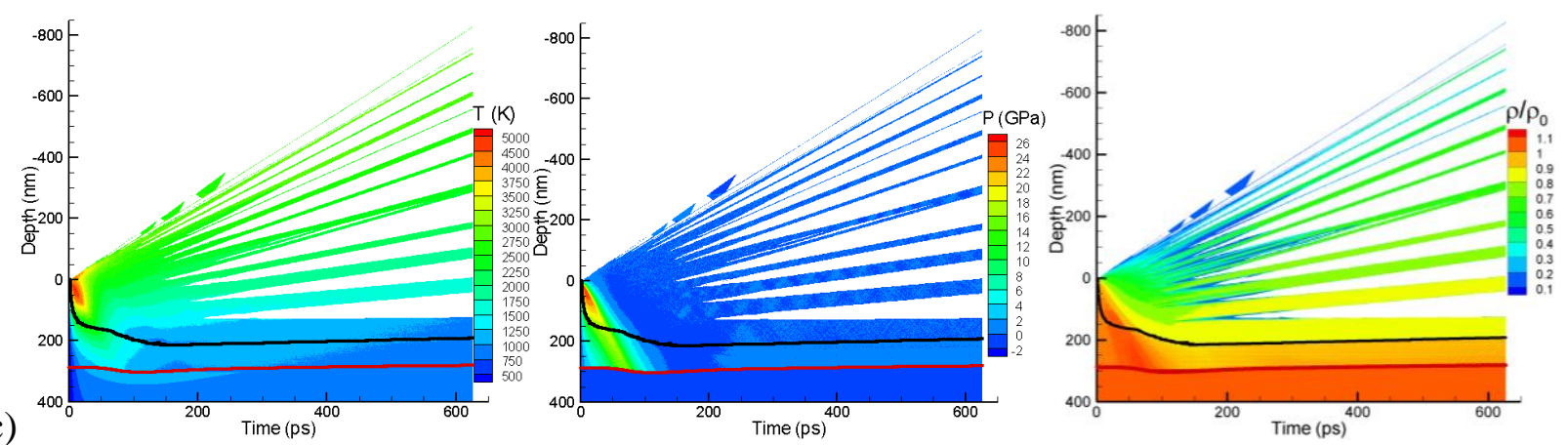

(d)
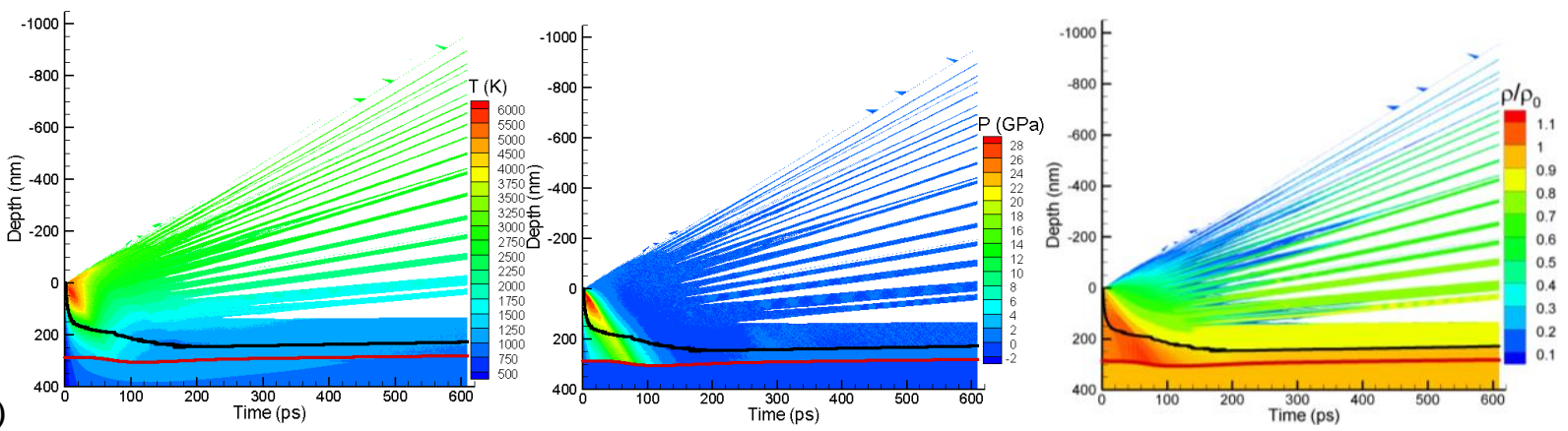

Figure 5.10: Contour plots of the lattice temperature, pressure and density as a function of time and depth for simulations of $100 \mathrm{fs}$ pulse laser irradiation of Ag (001) at absorbed fluences of (a) $0.15 \mathrm{~J} / \mathrm{cm}^{2}$, (b) $0.20 \mathrm{~J} / \mathrm{cm}^{2}$, (c) $0.25 \mathrm{~J} / \mathrm{cm}^{2}$, and (d) $0.30 \mathrm{~J} / \mathrm{cm}^{2}$. The black lines separate the melted regions from the crystalline bulk of the target. Red lines separate the MD and continuum parts of the combined TTM-MD model. The simulated system has the lateral size $L_{x} \sim 4.94 \mathrm{~nm}$ and $L_{y} \sim 9.88 \mathrm{~nm}$.

\subsection{Discussions}

In contrast to the experimental observation of surface swelling, significant material spallation sets in immediately when the voids are formed and grow to the size of the computational cell in the simulations. The failure to capture the experimentally observed surface swelling phenomenon in TTM-MD simulations is related to the two limitations of our computational setup. The first limitation is related to the small lateral size of the computational cell. As discussed above, the small lateral size leads to easy artificial spallation. In experiments, the lateral size can be thought of as infinity. If the lateral size is larger than the characteristic void size, the foamy structure with voids is expected to be frozen due to fast cooling, which can explain the observed surface swelling. Secondly, the uniform laser energy distribution and the 
periodic boundary conditions are applied in lateral directions (perpendicular to the direction of laser irradiation) in our computational setup. In experiments, the laser energy deposition more or less takes a Gaussian profile in lateral directions with a radially decaying intensity. Let us consider irradiation condition in which the peak fluence in the center of the laser spot is around the spallation threshold fluence, while the laser fluence is lower than the spallation threshold in the beam periphery. Under this condition, we can intuitively expect that the cold periphery of the irradiated spots can hold the hot central part, impede the complete separation of the liquid layer in the center of the laser spot from the target thus facilitating surface swelling. Keeping these limitations in mind, even though the surface swelling is not directly predicted in TTM-MD simulations, it is still possible to relate the simulation results with experimental observations.

First, the spallation threshold fluence found from the above simulations can be considered as a theoretical estimate of the threshold fluence for surface swelling in experiments. In fact, the simulated adsorbed fluence that corresponds to the spallation threshold is between $0.08 \mathrm{~J} / \mathrm{cm}^{2}$ and $0.09 \mathrm{~J} / \mathrm{cm}^{2}$. Converted to incident fluence by the Fresnel reflectivity of 0.969 , it is between $2.58 \mathrm{~J} / \mathrm{cm}^{2}$ and $2.90 \mathrm{~J} / \mathrm{cm}^{2}$, which agrees quantitatively with the swelling threshold of $2.5 \pm 0.1 \mathrm{~J} / \mathrm{cm}^{2}$ obtained in experiments.

Second, the experimental threshold (incident) fluence for material removal is $3.17 \pm 0.06$ $\mathrm{J} / \mathrm{cm}^{2}$, which is $\sim 16 \%$ higher than the spallation threshold fluence obtained from the simulations. This is also reasonably expected: the simulated spallation threshold fluence corresponds to the experimental swelling threshold as just discussed, and the experimental threshold fluence of material removal must be larger than the simulated spallation threshold. As seen in Fig. 5.3b, at the threshold incident fluence of $3.17 \pm 0.06 \mathrm{~J} / \mathrm{cm}^{2}$, the material removal happens in the top swollen part. On the other hand, the spallation is observed in the above simulations at the incident fluence of $3.17 \mathrm{~J} / \mathrm{cm}^{2}$ (adsorbed fluence $\sim 0.10 \mathrm{~J} / \mathrm{cm}^{2}$ ) as seen Figs. 5.8 and 5.9. After taking into account the two limitations of the simulations discussed above, the picture might be that the voids are formed due to the laser induced photomechanical effect, and the very top liquid layer is spallated while the foamy structure underneath is frozen with voids.

Third, the material removal below the original surface level at the very high laser fluences may be related to the combined contribution of photomechanical effects and phase explosion. From the simulations, the threshold of phase explosion is $\sim 0.25 \mathrm{~J} / \mathrm{cm}^{2}$ in adsorbed 
fluence or $\sim 8.1 \mathrm{~J} / \mathrm{cm}^{2}$ in incident fluence, which is comparable to the fluences at which a pronounced crater in the middle of the laser spot starts to develop in experiments, Fig. 5.3b.

From the above discussions of simulations and experiments, the following ablation mechanism is proposed and illustrated in Fig. 5.11. Below the adsorbed fluence $\sim 0.06 \mathrm{~J} / \mathrm{cm}^{2}$, no surface melting takes place. When the adsorbed laser fluence is between $\sim 0.06 \mathrm{~J} / \mathrm{cm}^{2}$ and $\sim 0.08$ $\mathrm{J} / \mathrm{cm}^{2}$, surface melting and resolidification occur without void formation or surface swelling. For the adsorbed laser fluence between $\sim 0.08 \mathrm{~J} / \mathrm{cm} 2$ and $\sim 0.10 \mathrm{~J} / \mathrm{cm}^{2}$, the voids are formed in the melted region due to the laser induced photomechanical effects. Some of the voids may be frozen during fast cooling, leading to the surface swelling. If the adsorbed laser fluence is greater than $0.10 \mathrm{~J} / \mathrm{cm}^{2}$, the surface swelling should similarly occur, but with the top swollen part removed by photomechanical spallation. When the adsorbed fluence is greater than $0.25 \mathrm{~J} / \mathrm{cm}^{2}$, atoms in the central part of the laser spot atoms are partly ablated through the phase explosion mechanism

(a)

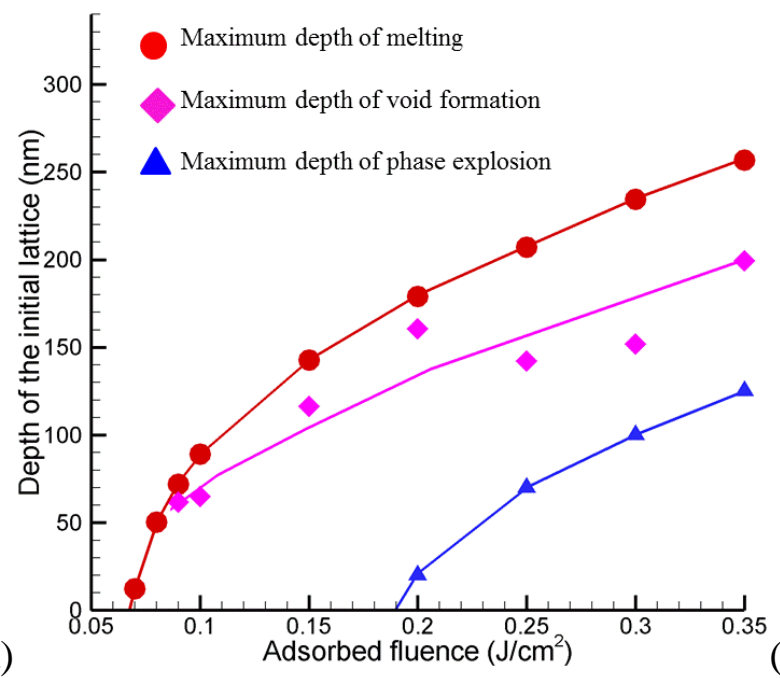

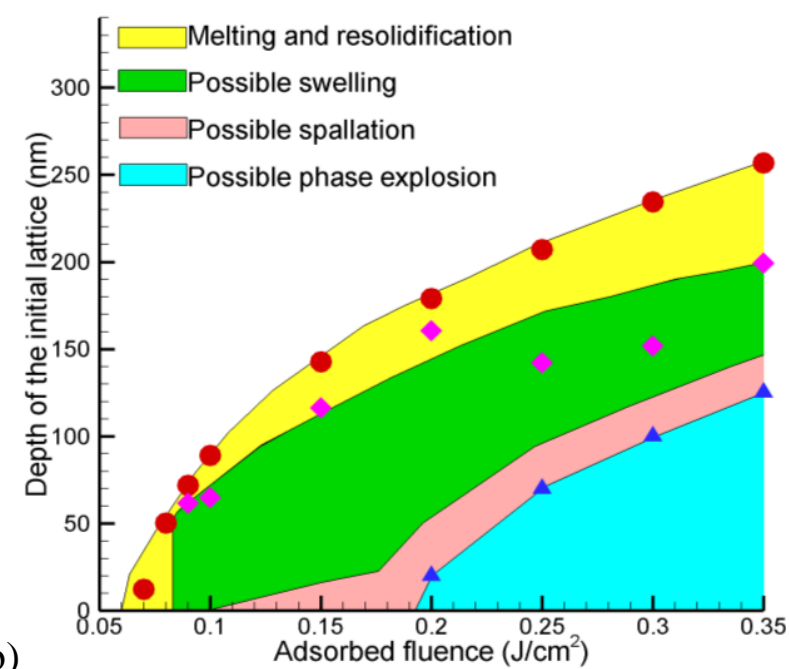

Figure 5.11: (a) The dependence of the maximum depth of melting, void formation and phase explosion on laser fluence obtained in TTM-MD simulations. (b) A map of ablation mechanisms proposed based on the comparison between the TTM-MD simulations and experiments. The maximum depth of phase explosion in (a) is defined as the maximum depth that reaches the threshold temperature for phase explosion, $3500 \mathrm{~K}$. The possible spallation region in (b) is not directly obtained from simulations, but a rough estimation based on experiment results shown in Fig. 5.3b. 


\section{Surface swelling and spallation in large-scale simulations of 100 fs laser irradiation of $\mathrm{Ag}(001)$}

\subsection{Introduction}

As discussed in the previous section, the failure to capture the experimental observation of surface swelling in the small-scale TTM-MD simulations is related to two limitations of the simulation setup. The first limitation is the small lateral size of the computational cell. The second one is neglect of the radially decaying laser energy intensity by using uniform laser energy distribution and periodic boundary conditions in the lateral directions. Both limitations result in easier spallation in simulations than in experiments. Due to the first limitation of using small lateral size of the computational cell, coalescence of several voids can lead to the percolation of voids across the computational cell and prompt ejection of the liquid materials. Due to the second limitation of neglecting the radially decaying laser intensity, the effect of the cold periphery restraining the central hot part from spallation is not taken into account. Intuitively, for a sufficiently large size of the laser spot, we expect the first limitation to be the dominant one, and the second limitation to play a secondary role. Therefore, to overcome the first limitation, we perform a series of large-scale simulations, in which we keep the same computational setup as in the simulations discussed in the last section, including the uniform laser energy distribution and periodic boundary conditions in the lateral directions, but increase the lateral size of the computational cell up to $L_{x}=L_{y}=98.7 \mathrm{~nm}$. With the depth of the MD part of the model being $L_{z}=150 \mathrm{~nm}$, the computational system consists of 84.2 million atoms. In fact, the use of the uniform laser energy distribution and periodic boundary conditions in the lateral directions is a good approximation of experimental conditions, as the lateral size of the computational cell is still much smaller than the laser spot size in Ref. [20] ( 100 nm compared to $\sim 30 \mu \mathrm{m}$ or $3 \times 10^{4} \mathrm{~nm}$ ).

In this section, the results of large-scale TTM-MD simulations of Ag (001) irradiated by a 100 fs laser pulse at a fluence close to the spallation threshold are presented and related to experiments. First, the threshold fluence of void formation is verified. Then two simulations at adsorbed fluences of $F=0.085 \mathrm{~J} / \mathrm{cm}^{2}$ and $F=0.09 \mathrm{~J} / \mathrm{cm}^{2}$ are discussed in detail. As discussed below, these two fluences correspond to different outcomes of laser irradiation: the adsorbed 
fluence $\mathrm{F}=0.085 \mathrm{~J} / \mathrm{cm}^{2}$ is above the void formation threshold but below the spallation threshold, while $\mathrm{F}=0.09 \mathrm{~J} / \mathrm{cm}^{2}$ is just above the spallation threshold. Finally, the experimentally observed surface swelling is explained based on the simulation results and the microscopic mechanisms of surface swelling are discussed.

\subsection{Threshold fluence for void formation}

As established in the last section for small-scale simulations, the "spallation" happens at $0.09 \mathrm{~J} / \mathrm{cm}^{2}$, but not at $0.08 \mathrm{~J} / \mathrm{cm}^{2}$. We argued that it is more appropriate to treat that "spallation" as "void formation" there due to the small lateral size of the computational cell. To more accurately determine the threshold fluence for void formation, 3 large-scale simulations are performed at $0.08 \mathrm{~J} / \mathrm{cm}^{2}, 0.085 \mathrm{~J} / \mathrm{cm}^{2}$, and $0.09 \mathrm{~J} / \mathrm{cm}^{2}$. The temperature, pressure, density, and collective velocity evolution in these three simulations is shown in Fig. 6.1 up to 200 ps. Fast energy deposition during the short pulse (100 fs) laser irradiation leads to fast heating of the surface region $(\sim 100 \mathrm{~nm})$ of the target. The fast heating under the condition of stress confinement (see section 5.3.3) results in the compressive stress build-up. The interaction of the compressive stresses with the free surface transforms them into an unloading tensile pressure wave propagating deeper into bulk target, and induces the collective motion of the top surface region away from the bulk of the target as seen in the velocity contour plot in Fig. 6.1. At the fluence greater than or equal to $0.085 \mathrm{~J} / \mathrm{cm}^{2}$, the tensile wave is strong enough to induce the voids formation as clearly seen by the lower (green) density region in the density contour plots, but not at the fluence $0.08 \mathrm{~J} / \mathrm{cm}^{2}$. Thus, the threshold absorbed fluence for void formation is more accurately determined in the range from $0.08 \mathrm{~J} / \mathrm{cm}^{2}$ to $0.085 \mathrm{~J} / \mathrm{cm}^{2}$. Converted to incident fluence using the Fresnel reflectivity of 0.969 , the threshold fluence for void formation is from $2.58 \mathrm{~J} / \mathrm{cm}^{2}$ to $2.74 \mathrm{~J} / \mathrm{cm}^{2}$, in quantitative agreement with the experiments, where the onset of swelling is observed at $2.5 \pm 0.1 \mathrm{~J} / \mathrm{cm}^{2}$ and spallation threshold is $3.17 \pm 0.06 \mathrm{~J} / \mathrm{cm}^{2}$ [20] Further investigation shows that $0.085 \mathrm{~J} / \mathrm{cm}^{2}$ is above the threshold of void formation, but below the spallation threshold, while $0.09 \mathrm{~J} / \mathrm{cm}^{2}$ is just above the spallation threshold, as discussed below. 

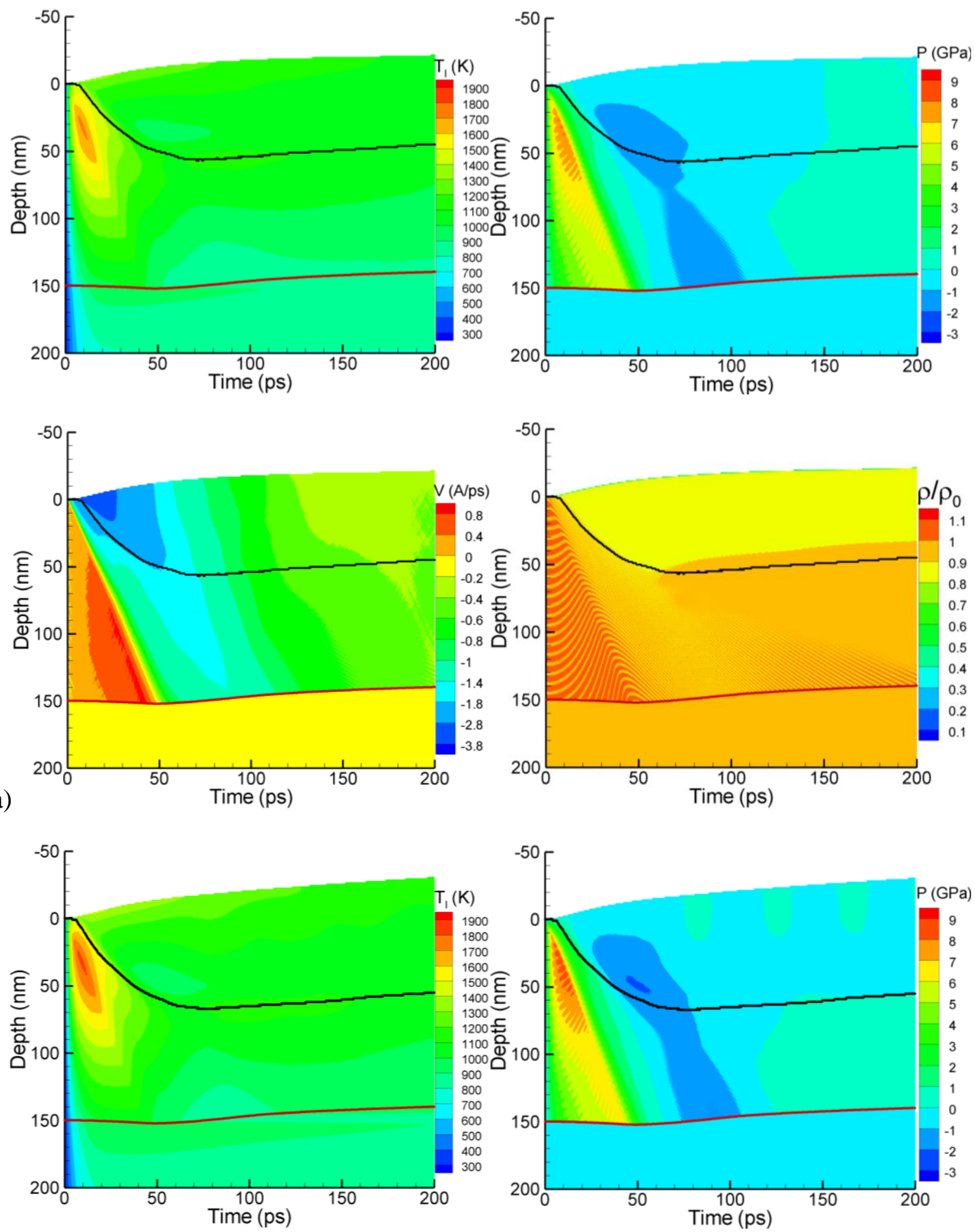
(b)
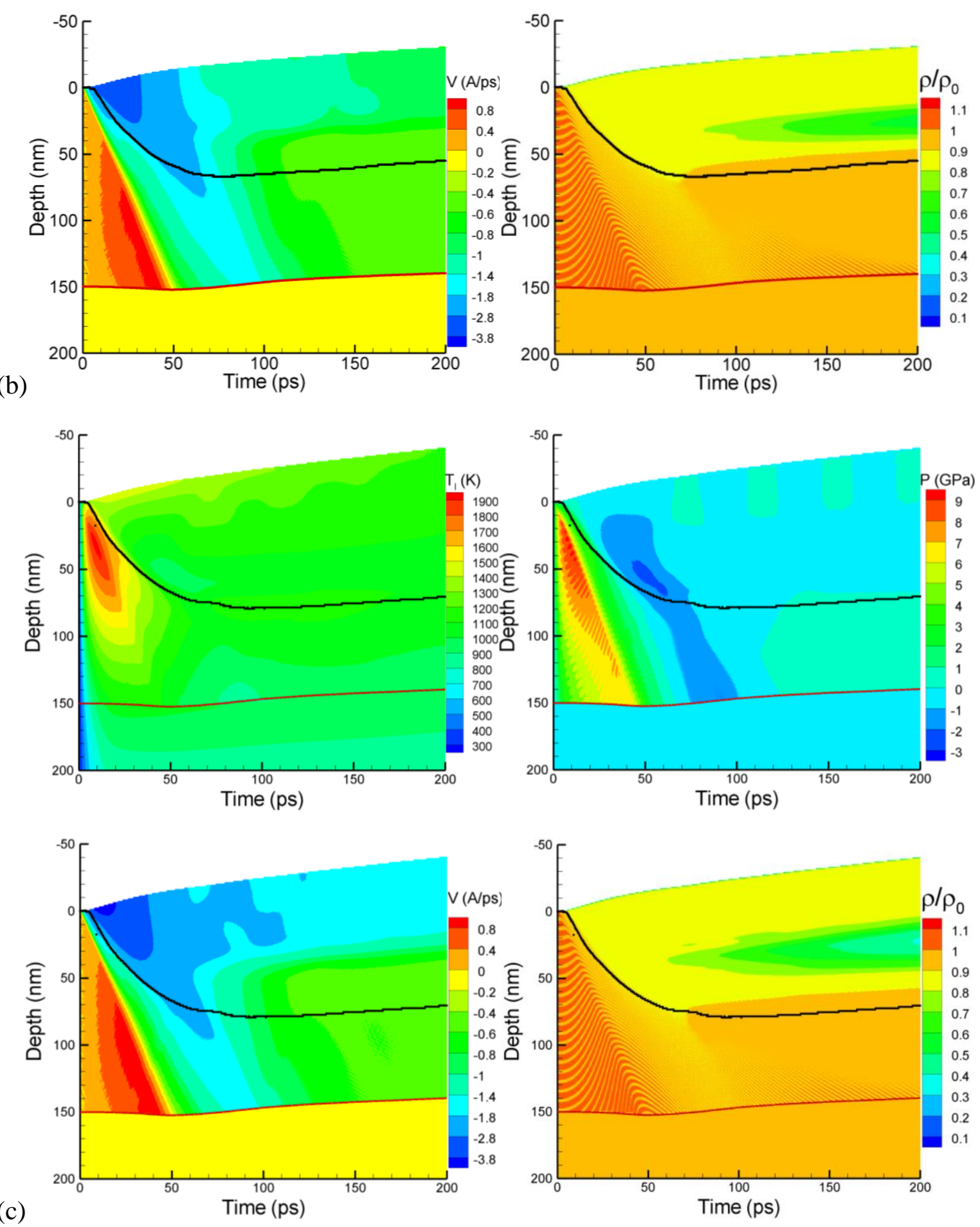

Figure 6.1: Contour plots of the lattice temperature, pressure, collective velocity in the direction normal to the surface, and density as a function of time after the laser pulse and depth under the initial surface for simulations of 100 fs pulse laser irradiation of $\mathrm{Ag}$ (001) target at absorbed fluences of (a) $0.08 \mathrm{~J} / \mathrm{cm}^{2}$, (b) $0.085 \mathrm{~J} / \mathrm{cm}^{2}$, and (c) $0.09 \mathrm{~J} / \mathrm{cm}^{2}$. Laser pulse is directed along the 
Y-axis, from the top of the contour plots. The black lines separate the melted regions from the crystalline bulk of the target. Red lines separate the MD and continuum parts of the combined TTM-MD model. The simulated system has the lateral size $L_{x}=L_{y}=98.7 \mathrm{~nm}$.

\subsection{Laser irradiation at $0.085 \mathrm{~J} / \mathrm{cm}^{2}$ : void formation, but no spallation}

In Fig. 6.2, the temperature, pressure, density and collective velocity evolution is shown up to $2 \mathrm{~ns}$ for the simulation performed at the fluence of $0.085 \mathrm{~J} / \mathrm{cm}^{2}$. Three important observations can be made based on Fig. 6.2. First, the voids are formed, grow, and coalescence, due to the propagation of the unloading tensile pressure wave, as evidenced by the pressure and density contour plots shown in Figs. 6.2b and 6.2d. Second, even though the low-density region affected by the void nucleation and growth contracts during the time between $800 \mathrm{ps}$ and $1200 \mathrm{ps,}$ most of voids are captured by the resolidification as seen in the density contour plot Fig. 6.2d. Finally, the homogeneous crystal nucleation occurs in the top surface region $(\sim 30 \mathrm{~nm})$ as the temperature drops down to $\sim 790 \mathrm{~K}\left(0.69 \mathrm{~T}_{\mathrm{m}}\right)$, as seen from the sudden temperature increase in the part of the temperature contour plot, marked by the blue square in Fig. 6.2a. In the following, these three observations are studied in more details. 
(a)

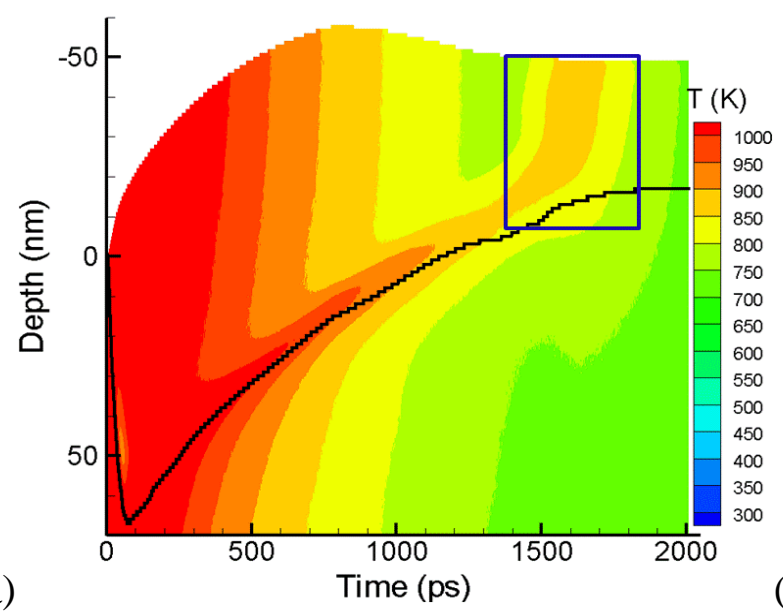

(c)

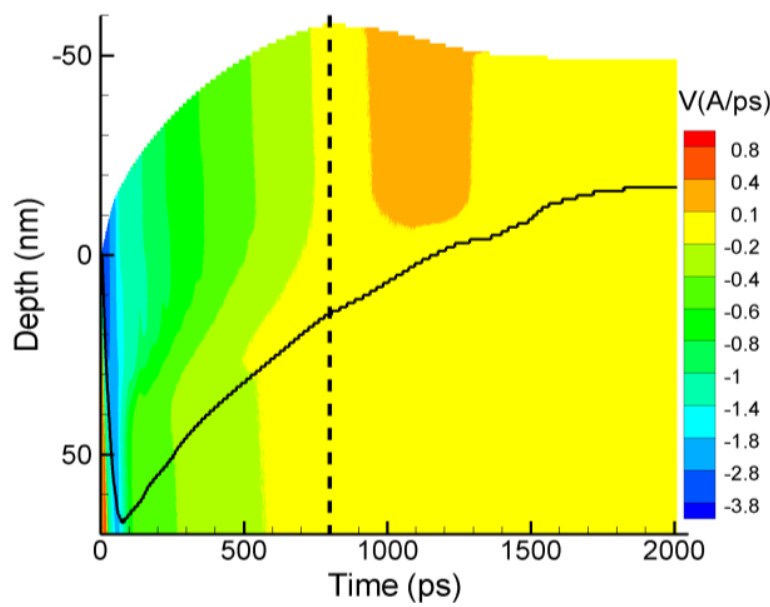

(b)
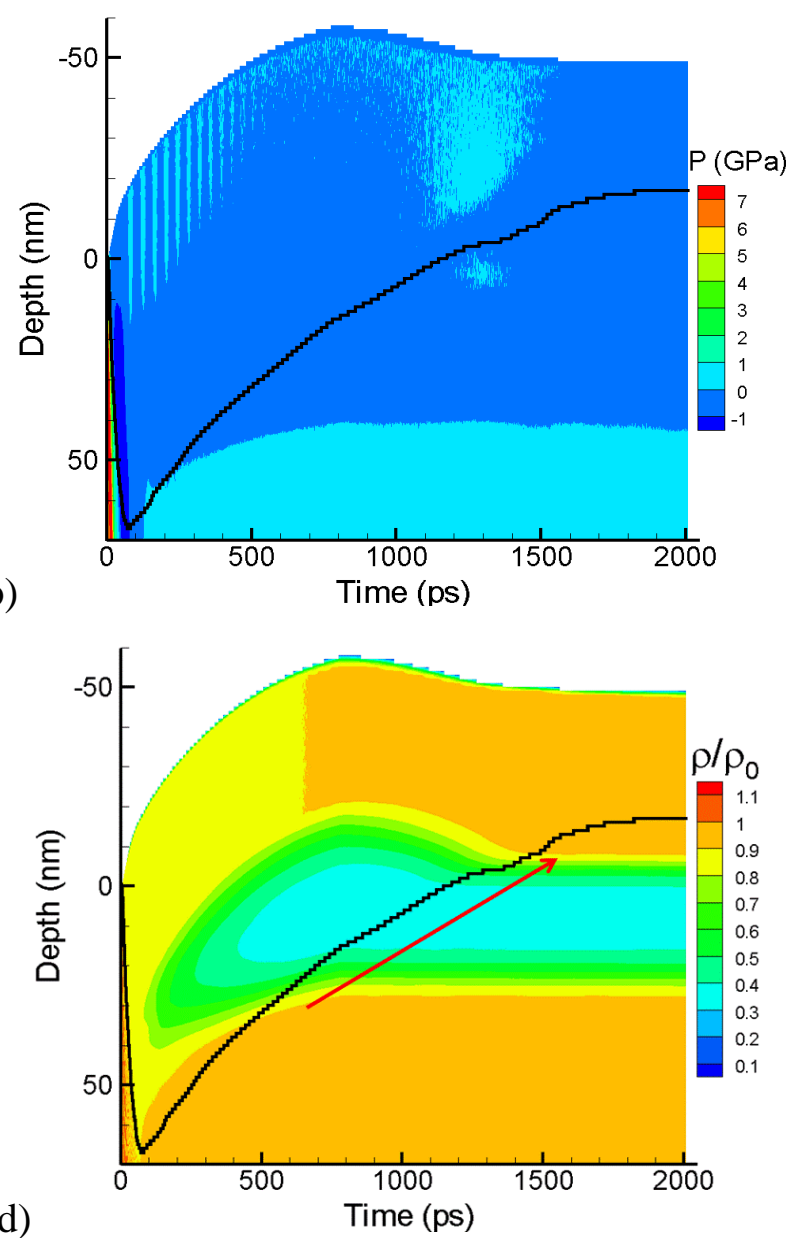

Figure 6.2: Contour plots showing the evolution of (a) the lattice temperature, (b) pressure, (c) collective velocity in the direction normal to the surface, and (d) density normalized to the density of $\mathrm{Ag}$ at $300 \mathrm{~K}$ before the irradiation as a function of time and depth in the surface region $(<70 \mathrm{~nm}$ ) for simulations of $100 \mathrm{fs}$ pulse laser irradiation of Ag (001) target at an absorbed fluences of $0.085 \mathrm{~J} / \mathrm{cm}^{2}$. The black lines separate the "melted regions" from the crystalline bulk of the target. The blue rectangle in the temperature contour plot (a) marks the region of homogeneous nucleation of multiple crystallites in the surface region of the target. The black dashed line in (c) shows the time when the collective velocity drops down to 0 . The red arrow indicates the resolidification crossing the region of voids. 


\subsubsection{Void nucleation, growth, coalescence and contraction}

As discussed above, voids are formed in the melted material due to the laser-induced photomechanical effect. The void evolution is presented in Fig. 6.3, where the voids are viewed from the top. The void evolution can also be illustrated through the evolution of void number and void volume as shown in Fig. 6.4. Under conditions of the large-scale simulation performed with fluence of $0.085 \mathrm{~J} / \mathrm{cm}^{2}$, four distinct regimes can be distinguished in the void evolution: void nucleation, void growth/ coalescence, void contraction, and void captured by the resolidification front. First, the interaction of the unloading tensile pressure wave with the mechanically-weak melted material results in the "void nucleation" as indicated by the fast increase of the void number and void volume during the time period from $\sim 20$ ps to $\sim 60$ ps as seen in in Fig. 6.4. The second stage of "void growth/coalescence" starts from $\sim 60$ ps and continues up to $\sim 800$ ps. During this stage, the number of small voids is steadily decreasing, whereas large voids continue to grow and coalesce. Thus, the total volume of voids increases, and the total number of voids decreases as seen in Fig. 6.4 for the time between $\sim 60$ ps and $\sim 800$ ps. At the end of this stage, only five big voids exist, with the average radius of $\sim 22 \mathrm{~nm}$. The driving force of voids volume increase is the kinetic energy of the motion of top surface layer away from the bulk target (negative velocity) seen in the collective velocity contour plot in Figs. 6.1c and 6.2c. At the late stage of the void expansion, the increase of the (liquid) surface energy related to the voids volume increase slows down the collective motion of the top surface layer and acts as a resistance to spallation. As soon as the relative velocity of the top surface layer (above voids) drops down to 0 at $\sim 800$ ps (as indicated by the black dashed line in the Fig. 6.2c, the second stage of the "void growth/coalescence" ends and the void evolution comes into the third stage of "void contraction". At this stage, the number of voids stays the same, while the voids volume decreases indicating the voids contraction. The voids contraction is driven by the liquid surface energy minimization. At the same time, the resolidfication front crosses the region of voids and inhibits the further voids contraction. That is why we only see the voids contraction on the side close to the free surface, where the material is still in liquid state, in the contour plot shown in Fig. 6.2d. Finally in the fourth stage, the voids are captured after the resolidification front crosses the region of voids as discussed below. 


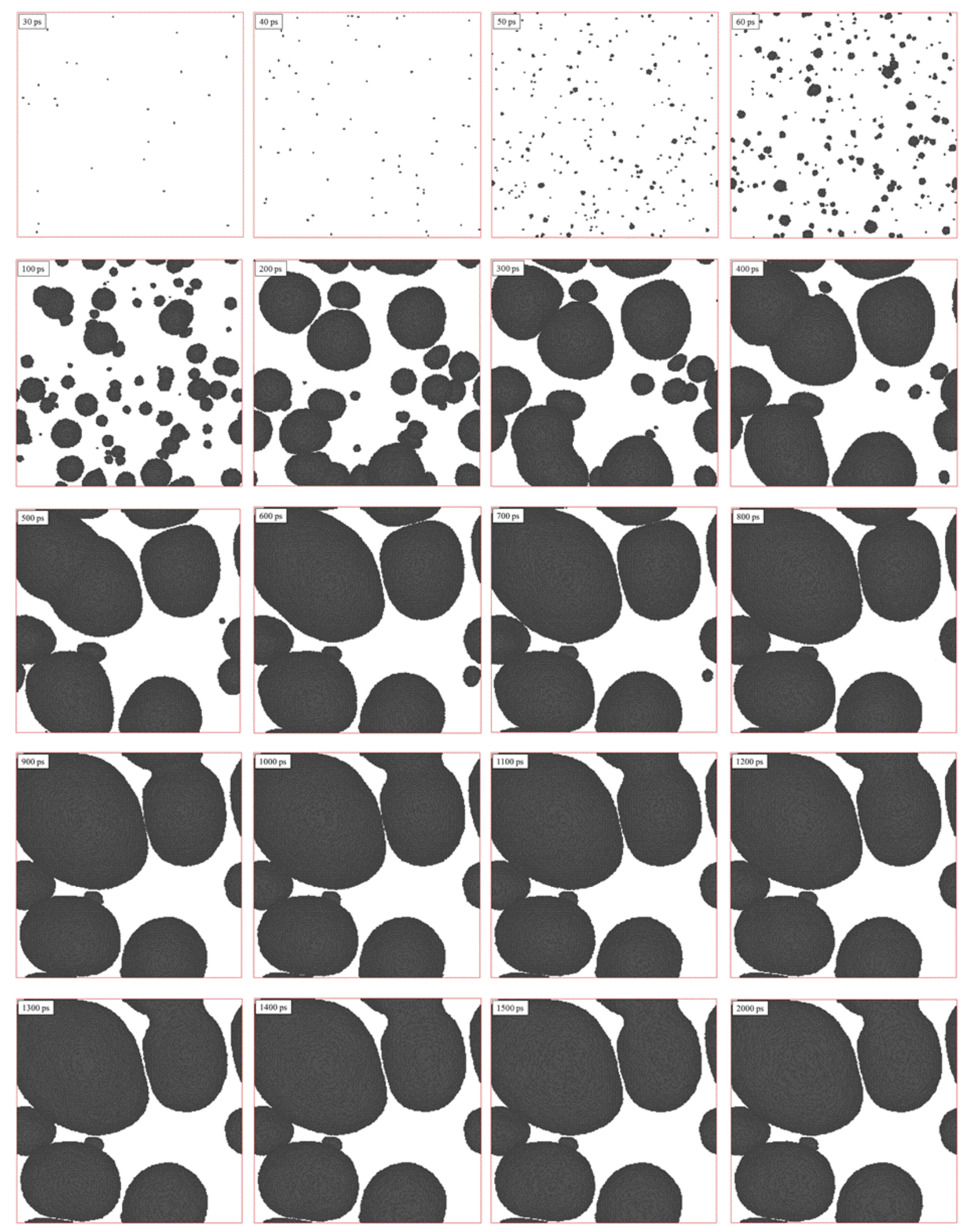


Figure 6.3: Snapshots viewed from top, showing the void nucleation, growth, and coalescence. To identify voids, the computational system is divided into cubic cells of $0.4 \mathrm{~nm}$ and cells without atoms inside are found and plotted.

(a)
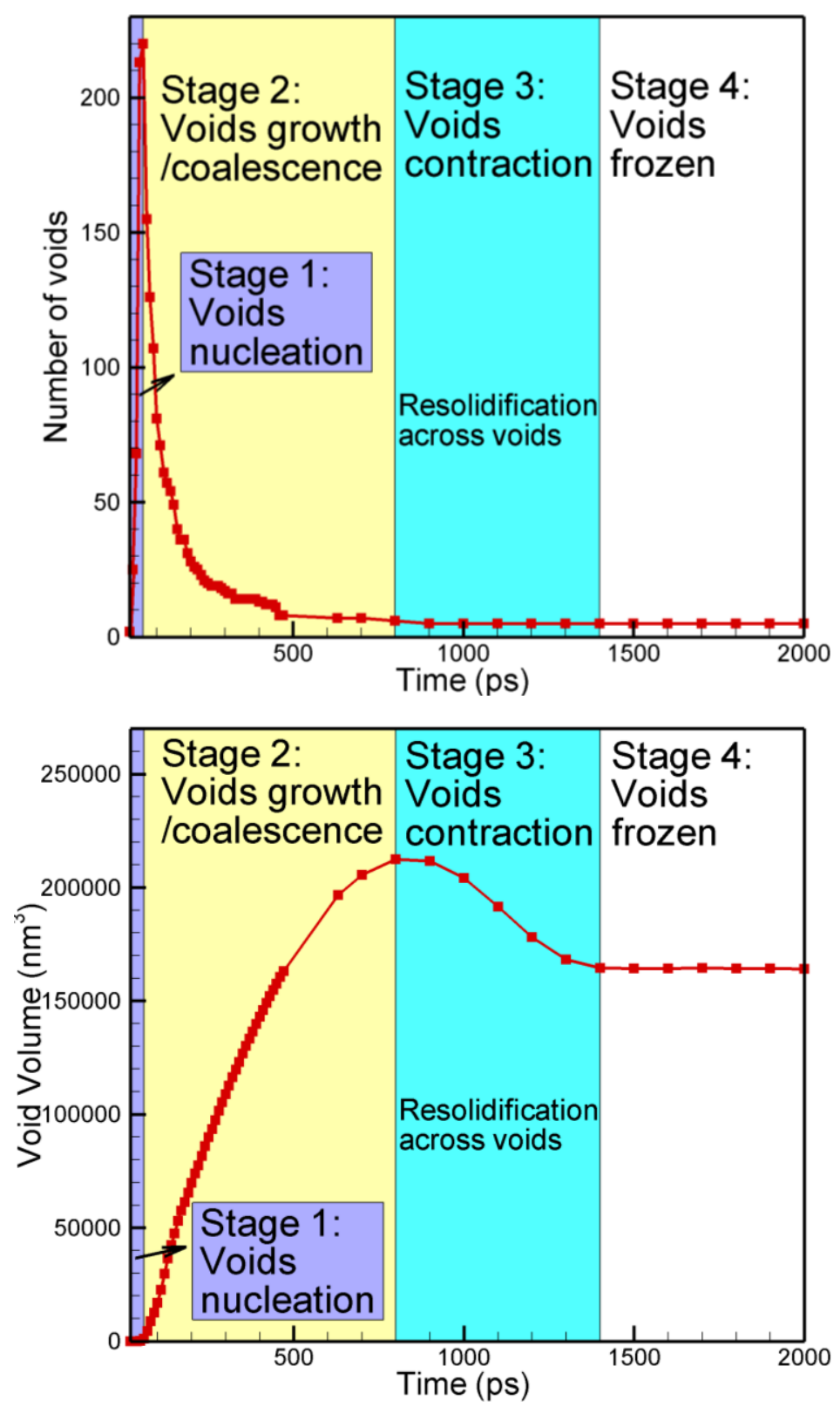

(b)

Figure 6.4: Number of voids (a) and the total volume of voids (b) as a function of time after the laser irradiation at a fluence of $0.085 \mathrm{~J} / \mathrm{cm}^{2}$. Voids are defined as clusters of connected empty cubic cells as shown in Fig. 6.3. Four stages of void evolution are indicated. 


\subsubsection{Voids captured (frozen) by heterogeneous resolidification}

The strong temperature gradient and the fast electronic heat conduction in Ag lead to the rapid cooling of the surface region of the irradiated target. By the time of $100 \mathrm{ps}$, the temperature in the liquid-solid interface drops below the equilibrium melting temperature of $\mathrm{Ag}$, which activates the resolidification process as seen in Fig. 6.2. This resolidification is of heterogeneous nature, i.e., it is epitaxial regrowth of crystal structure from the bulk of the target. The resolidification front moves with a high velocity of $\sim 100 \mathrm{~m} / \mathrm{s}$ until $\sim 500 \mathrm{ps}$, when it meets the bottom of the region with voids. The front then slows down and takes $\sim 700$ ps to cross the whole region of voids (up to $\sim 1200$ ps). In Fig. 6.5, a series of snapshots are shown in order to illustrate this resolidfication process. The atomistic picture of resolidification front crossing the region of voids is clearly seen. After the resolidification front crosses the region with voids, the number of voids and their total volume stay constant as seen in Fig. 6.4.

The observation that the resolidfication freezes the foamy structure of voids, provides a clue for explaining the experimental observation of surface swelling and the voids formation during short pulse laser irradiation [19,20]. Quantitatively, the total void volume of $\sim 1.7 \times 10^{5}$ $\mathrm{nm}^{3}$ accounts for the surface swelling by $\sim 17 \mathrm{~nm}$, much smaller than experimentally observed surface swelling of several hundreds of nanometers at an incident fluence of $3.17 \mathrm{~J} / \mathrm{cm}^{2}$. But this is expected, as the simulated adsorbed fluence of $0.085 \mathrm{~J} / \mathrm{cm}^{2}$ corresponds to the incident fluence of $2.74 \mathrm{~J} / \mathrm{cm}^{2}$ after taking into account the Fresnel reflectivity of 0.969 , much less than 3.17 $\mathrm{J} / \mathrm{cm}^{2}$. In fact, no obvious surface swelling is observed in experiments, probably due to the fact that the very small swelling of $\sim 17 \mathrm{~nm}$ is on the order of surface roughness and cannot be detected by AFM microscopy. This issue will be discussed in more details in section 6.5.1. 

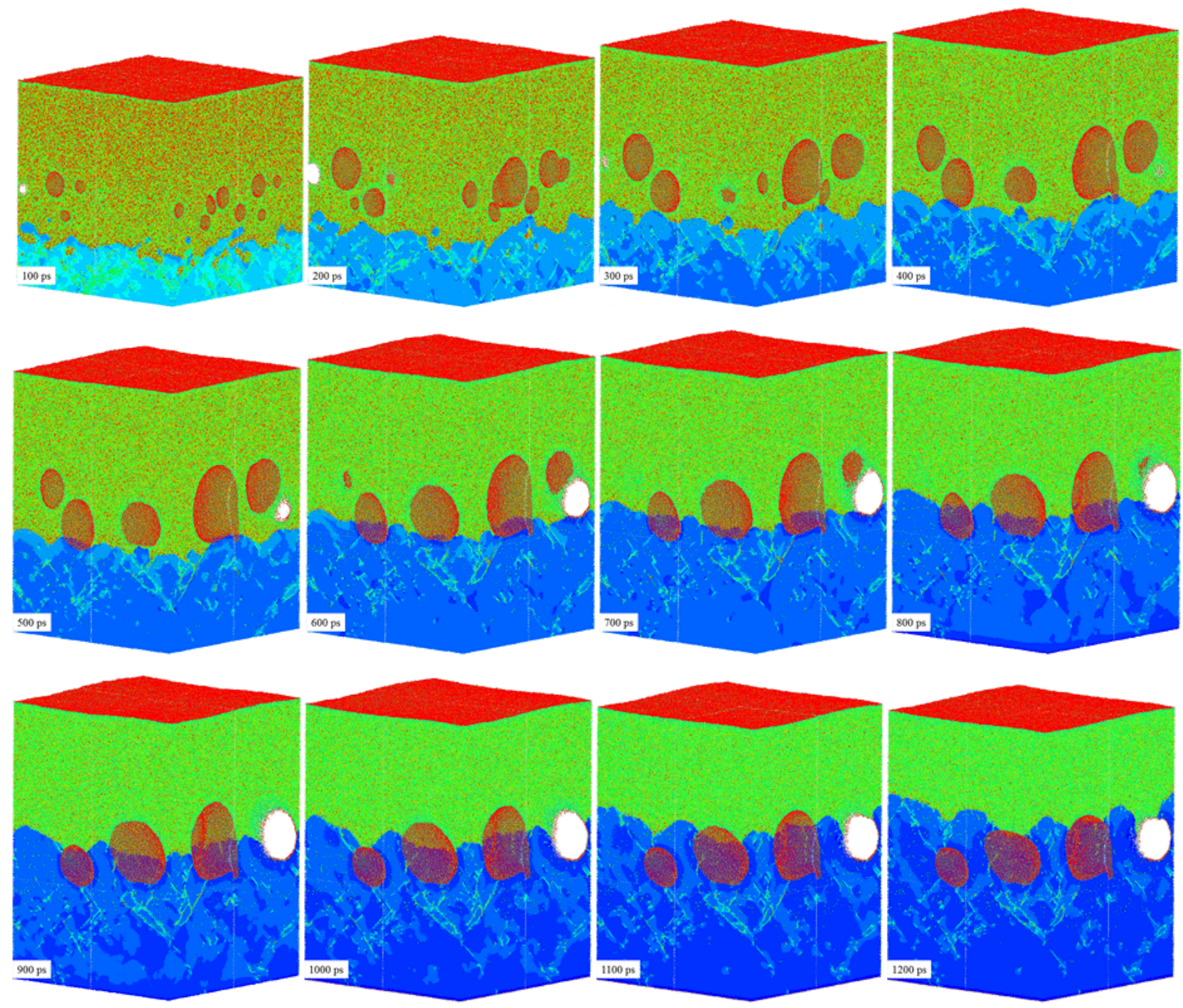

Figure 6.5: A series of snapshots of atomic structure in the surface region $(<80 \mathrm{~nm})$ illustrating the resolidification process in the simulation of $100 \mathrm{fs}$ pulse laser irradiation of $\mathrm{Ag}(001)$ target at a fluence of $0.085 \mathrm{~J} / \mathrm{cm}^{2}$. The snapshots are shown for the times from from 100 ps to $1200 \mathrm{ps}$ with the time interval of $100 \mathrm{ps}$. To reduce the thermal noise in atomic positions, the configurations are quenched for $0.2 \mathrm{ps}$. The atoms are colored by their potential energy with the scale from $-2.85 \mathrm{eV}$ to $-2.64 \mathrm{eV}$. The blue atoms have lower energy, indicating the solid structure, while the green atoms have higher energy, indicating the liquid structure. The red atoms are at the free surface and have the highest energy due to the contribution of the surface energy. The resolidification front separating the liquid from crystal is clearly seen. 


\subsubsection{Homogeneous crystal nucleation to form nano-crystalline structure}

During the heterogeneous resolidification process discussed above, the top surface layer is further cooled. When the temperature is dropped down to $\sim 790 \mathrm{~K}(69 \%$ of equilibrium melting temperature of $\mathrm{Ag}$ ) at $~ 1300$ ps, several small crystalline clusters are homogeneously nucleated in the top surface layer. From $\sim 1400$ ps, this homogeneous resolidification is accelerated and it takes only $\sim 200$ ps to solidify the whole top $\sim 30 \mathrm{~nm}$ layer. Since the resolidification is an exothermic process, heat is released in the surface region of the target which leads to a sudden temperature increase during this homogeneous resolidification, as seen in the region marked by the blue rectangle in the temperature contour plot shown in Fig. 6.2a. In order to illustrate the homogeneous resolidification process, a series of side-view snapshots are shown in Fig. 6.6 and cross sections cut at the depth of $35 \mathrm{~nm}$ from the initial surface are shown in Fig. 6.7. From Figs. 6.6 and 6.7, it is clearly seen that nuclei appear randomly, then grow bigger and meet with each other to generate a $\sim 30 \mathrm{~nm}$ layer of nano-crystalline structure.
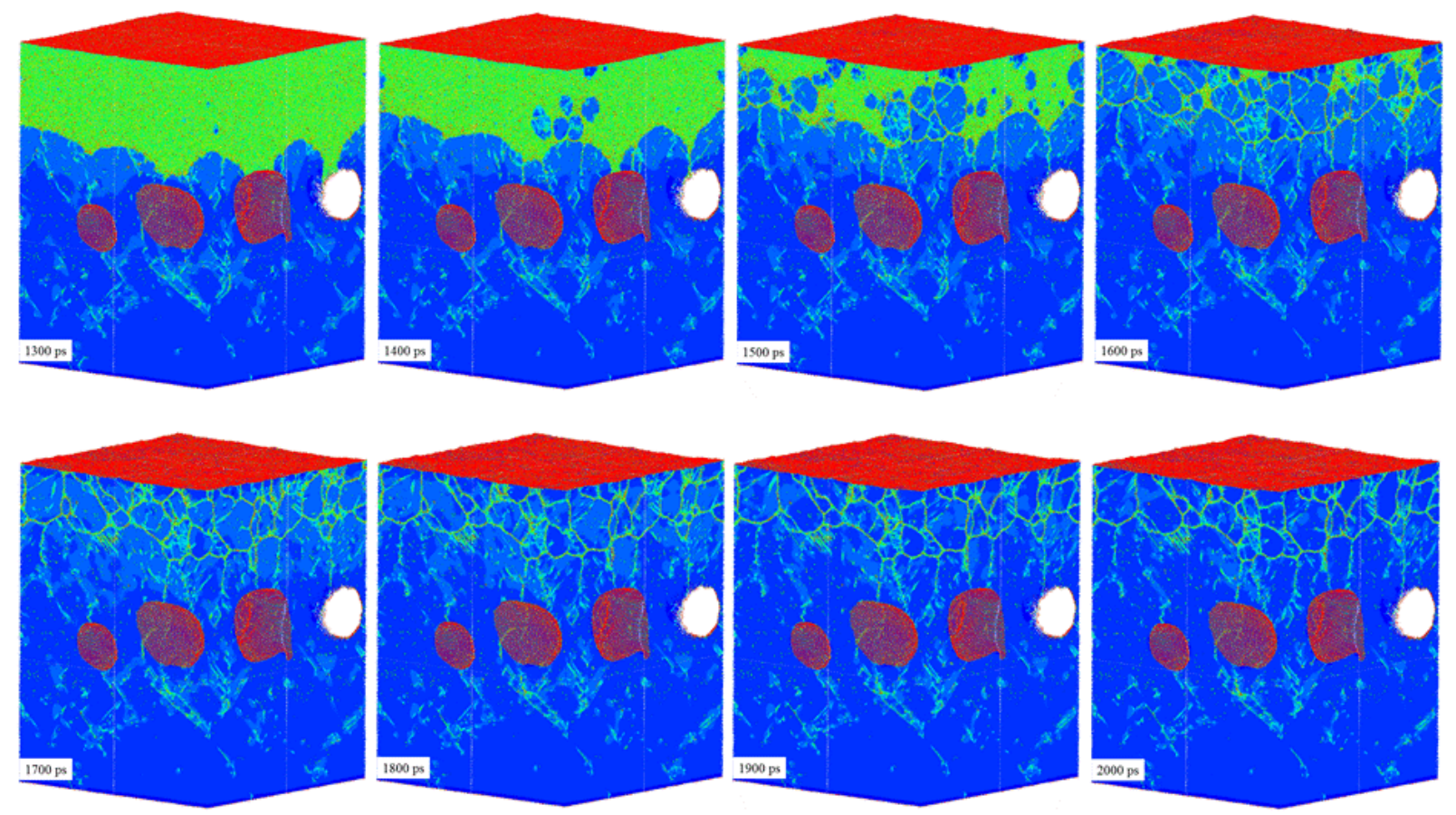

Figure 6.6: Snapshots from the simulation of 100 fs pulse laser irradiation of Ag (001) target at a fluence of $0.085 \mathrm{~J} / \mathrm{cm}^{2}$ shown from $1300 \mathrm{ps}$ to $2000 \mathrm{ps}$ to illustrate the homogeneous resolidification process and the generation of nano-crystalline surface structure. The atoms are colored by their potential energy as in Fig. 6.5. The grain boundaries separating different nanograins can be clearly identified as lines of high energy atoms. 

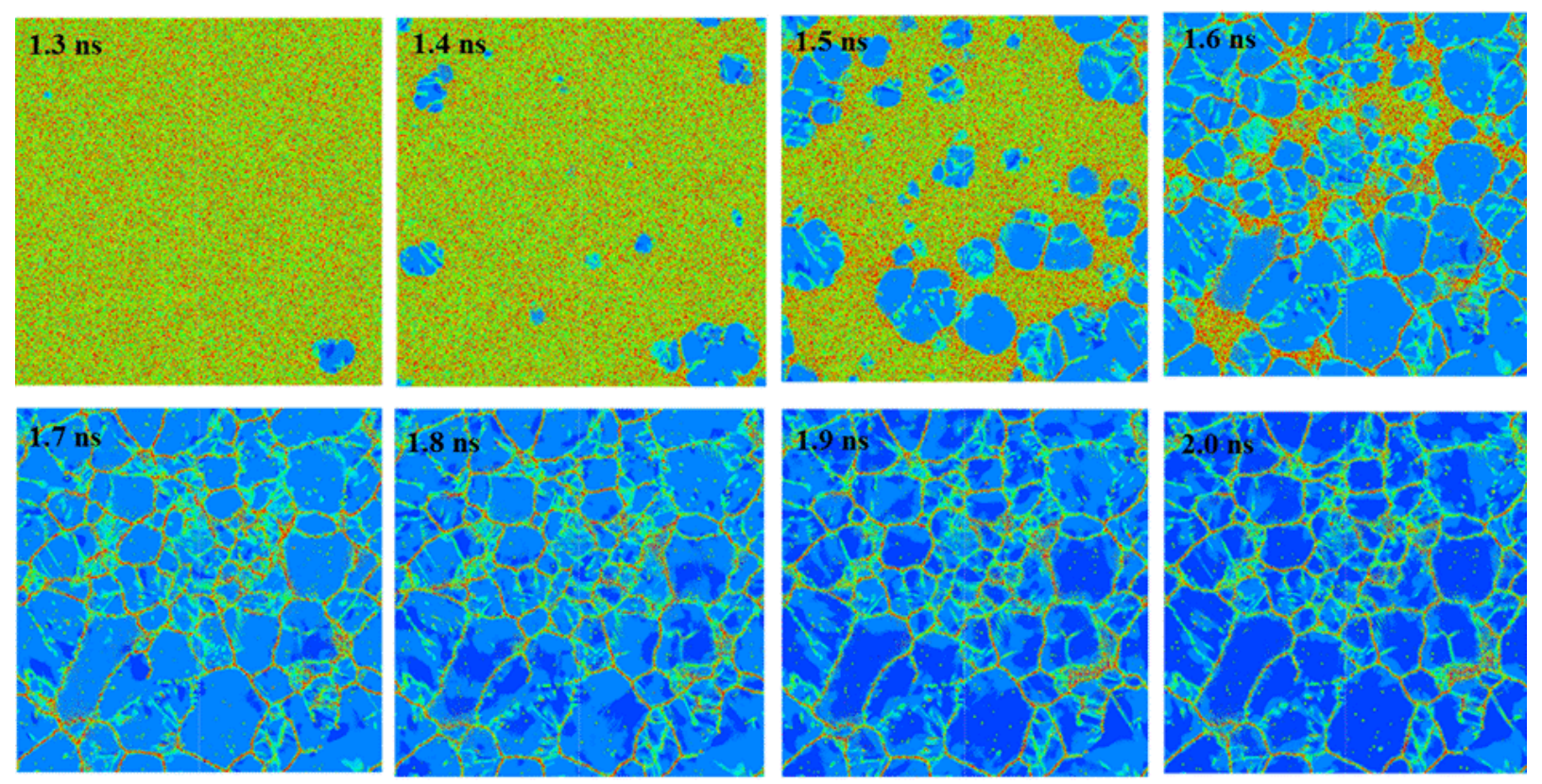

Figure 6.7: Cross sections cut at the depth of $30 \mathrm{~nm}$ from the initial surface for the snapshots shown in Fig. 6.6 to illustrate the homogeneous resolidification process and the generation of nano-crystalline surface structure. The atoms are colored by their potential energy. The grain boundaries separating different nano-grains can be clearly identified as lines of high energy atoms.

\subsubsection{Microstructure of the top surface layer}

The structural analysis is performed for the final atomic configuration at $2 \mathrm{~ns}$ following the method suggested in [80] with some modifications [79], in which each atom is identified as FCC, HCP, BCC, or "defect" by its local structural environment. As shown in Figs. 6.8a and $6.8 \mathrm{~b}$, all nano-grains have close-packed (FCC/HCP) structure. The angle between the normal to the close-packed plane and the [001] direction of the original FCC lattice is calculated for each nano-grain as shown in Fig. 6.8c. The $<111>$ pole figure in Fig. 6.8d is obtained by collecting stereographic projections of four $<111>$ directions of all FCC atoms in the nano-crystalline layer. Due to the random nature of the homogeneous nucleation, the nano-grains are randomly oriented as shown in Figs. $6.8 \mathrm{c}$ and $6.8 \mathrm{~d}$, and no texture can be identifed. The nano-grains have the radius on the order of several nanometers, as shown in Fig 6.8d, where the radius is defined for spheres that have the actual volume of the nano-grains.

Each nano-grain can be represented as a stack of close-packed planes. It is well known that different stacking sequences result in different close-packed structures, i.e., FCC structure is 
generated by stacking of the close-packed planes in the sequence of $\bullet \bullet A B C A B C A B C \cdot \bullet \bullet$, while HCP corresponds to the sequence of $\bullet A \mathrm{ABABAB} \bullet \bullet$ as illustrated in Fig. 6.9. Under the condition of strong undercooling realized in the current simulation, the homogeneous nucleation and growth occur so fast that the stacking does not always follow the FCC sequence that corresponds to the equilibrium Ag crystal. That is why we see a mixture of FCC planes with HCP planes in each nano grain, as identified by our structural analysis. The HCP planes can be thought of as the stacking faults or twins in the FCC lattice as summarized in Table 2.

(a)

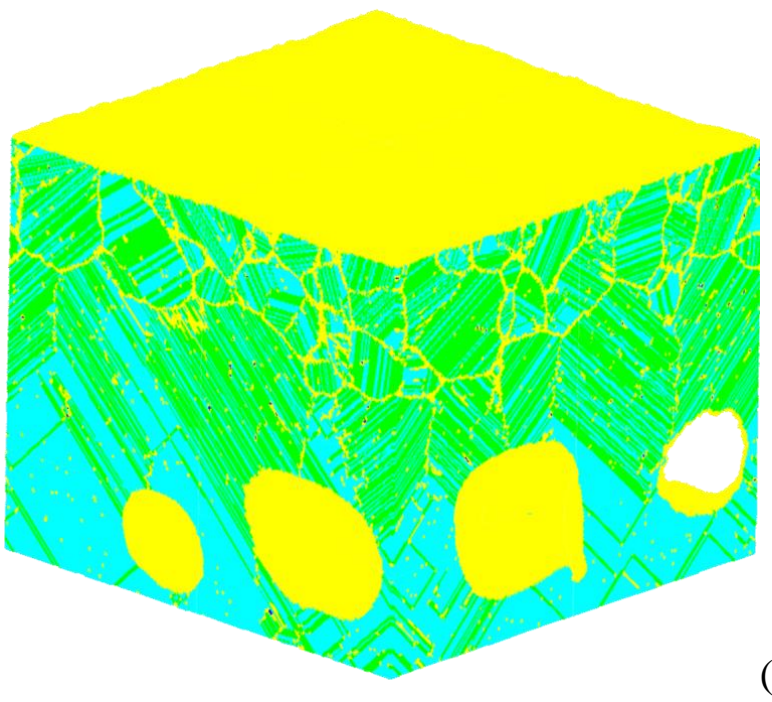

(b)

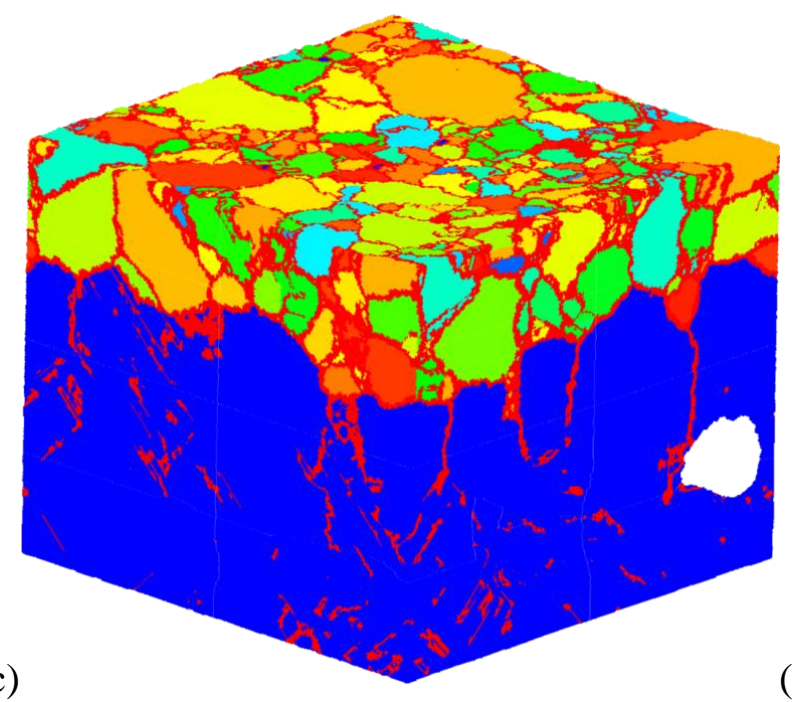

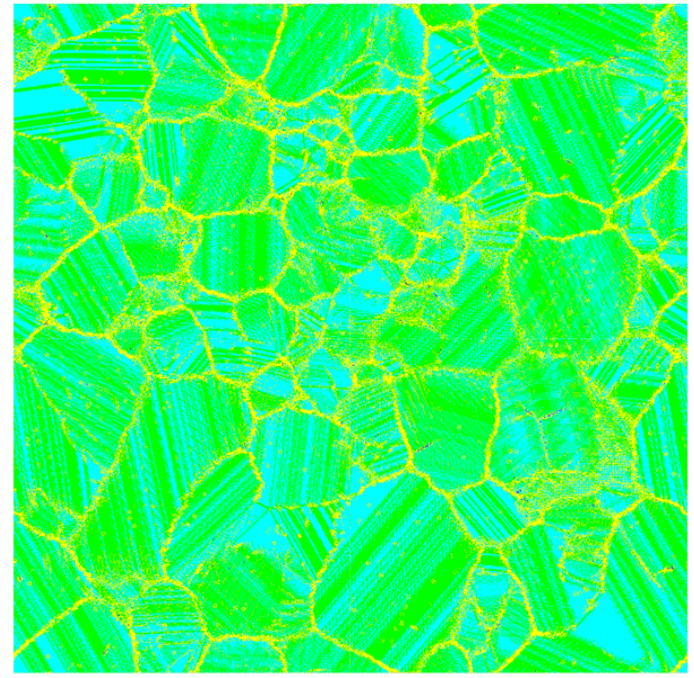

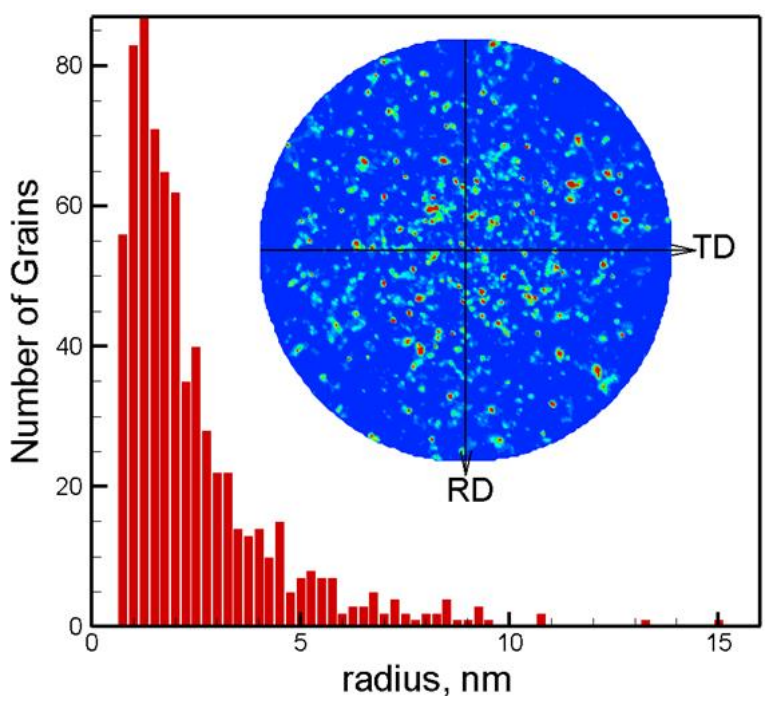

Figure 6.8: (a) Atomic configuration at $2 \mathrm{~ns}$ with atoms colored by their local structural environment: light blue for FCC atoms, green for HCP atoms, blue for BCC atoms and yellow for defect atoms. (b) Cross section cut at the depth of $35 \mathrm{~nm}$ from the initial surface with atoms colored by their local structural environment. Yellow lines indicate the grain boundaries. (c) 
Atomic configuration at $2 \mathrm{~ns}$ with atoms colored by the angle between the normal to the closepacked planes in the corresponding grain and the [001] direction of the original FCC lattice. Blue atoms in the bottom part indicate that they belong to the region that experienced epitaxial resolidification. Red atoms belong to the grain boundaries. (d) The nano-grain size distribution indicating the nano-grains have sizes of several nanometers in radius and the $\langle 111\rangle$ pole figure indicating the random orientations of the nano-grains.

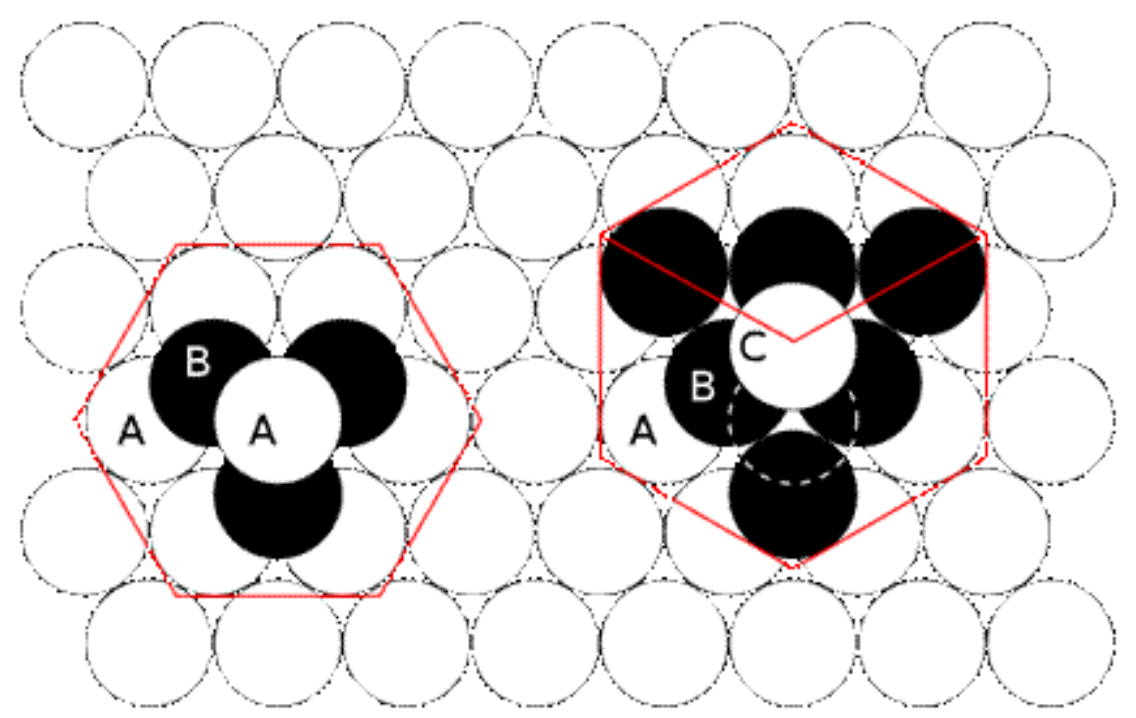

Figure 6.9: Different stacking of close-packed planes to form HCP lattice (left) and FCC lattice (right).

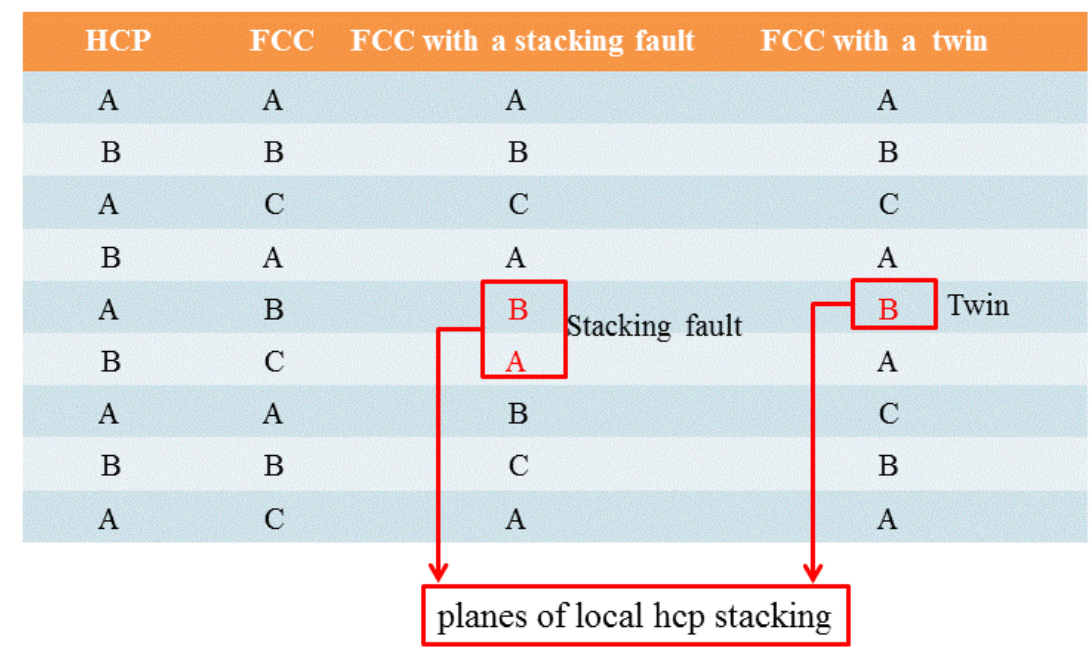

Table 2: Stacking sequence of close-packed planes in the lattice of HCP, FCC, FCC with a stacking fault and FCC with a twin. 
As we know, there are four $\{111\}$ close-packed planes in a FCC lattice which forms a specific-oriented tetrahedron. A twin in the FCC lattice can be represented as a single "HCP" plane inserted into the FCC crystal. The FCC stacking sequence changes after crossing the twin, from $\cdots A B C A B C \cdots$ to $\cdots$ ACBACB $\bullet$ as shown in Table 2. Thus, the FCC lattice on one side of the twin has a different tetrahedron from that of the FCC lattice on the other side. In fact, the FCC tetrahedron is rotated by 60 degrees around the normal direction to the twin plane as seen in Fig. 6.10. As for a stacking fault, it consists of two consecutive "HCP" planes in the FCC lattice as seen in Table 2. The FCC stacking sequences are exactly the same on both sides of the stacking fault, such as $\bullet \bullet A B C A B C \bullet \bullet$ shown in Table 2. Thus, the FCC lattices on both sides of the stacking fault have exactly the same tetrahedron. This can be explained by the fact that the tetrahedron returns back to its original position after two consecutive rotations by 60 degrees around the same axis. More generally, the FCC tetrahedron keeps the same orientation after crossing even number of parallel "HCP" planes, and is rotated by 60 degrees after crossing odd number of parallel "HCP" planes. Therefore, we can generally treat even number of (consecutive) parallel "HCP" planes as a stacking fault (or a sequence of stacking faults) and odd number of parallel "HCP" planes as a twin boundary. In Fig. 6.11, a small region of the nanocrystalline structure obtained in the simulation is given to illustrate the close-packed structure with stacking faults and twins.

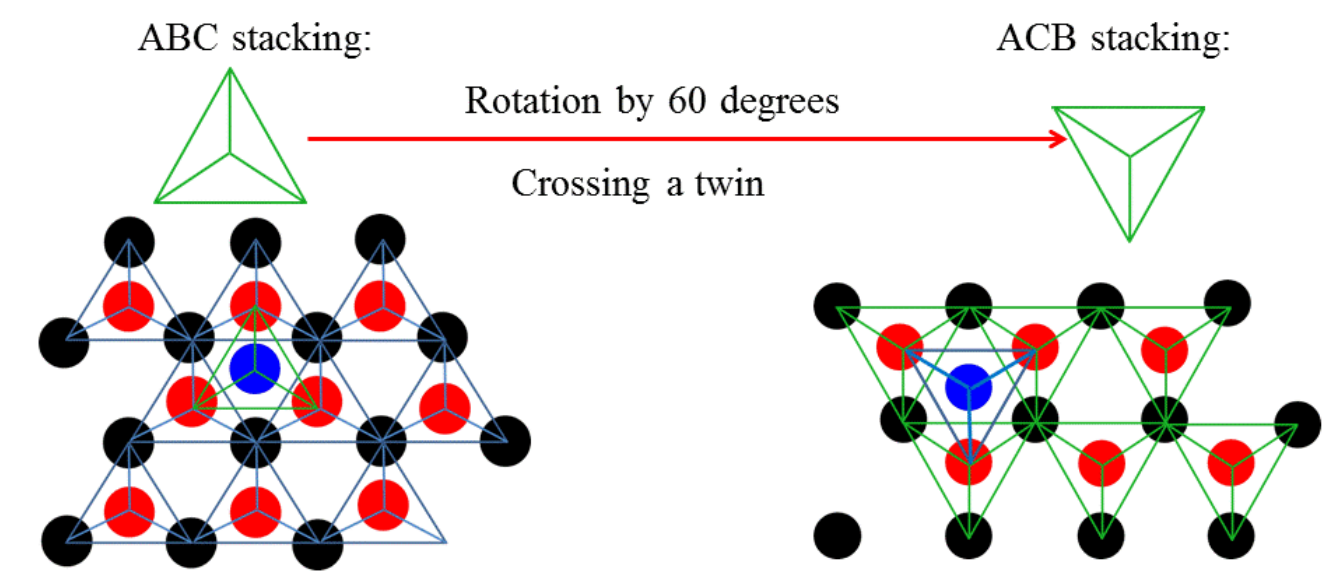

Figure 6.10: Change of FCC stacking sequence after crossing a twin and the corresponding change in the orientation of the FCC tetrahedron. 


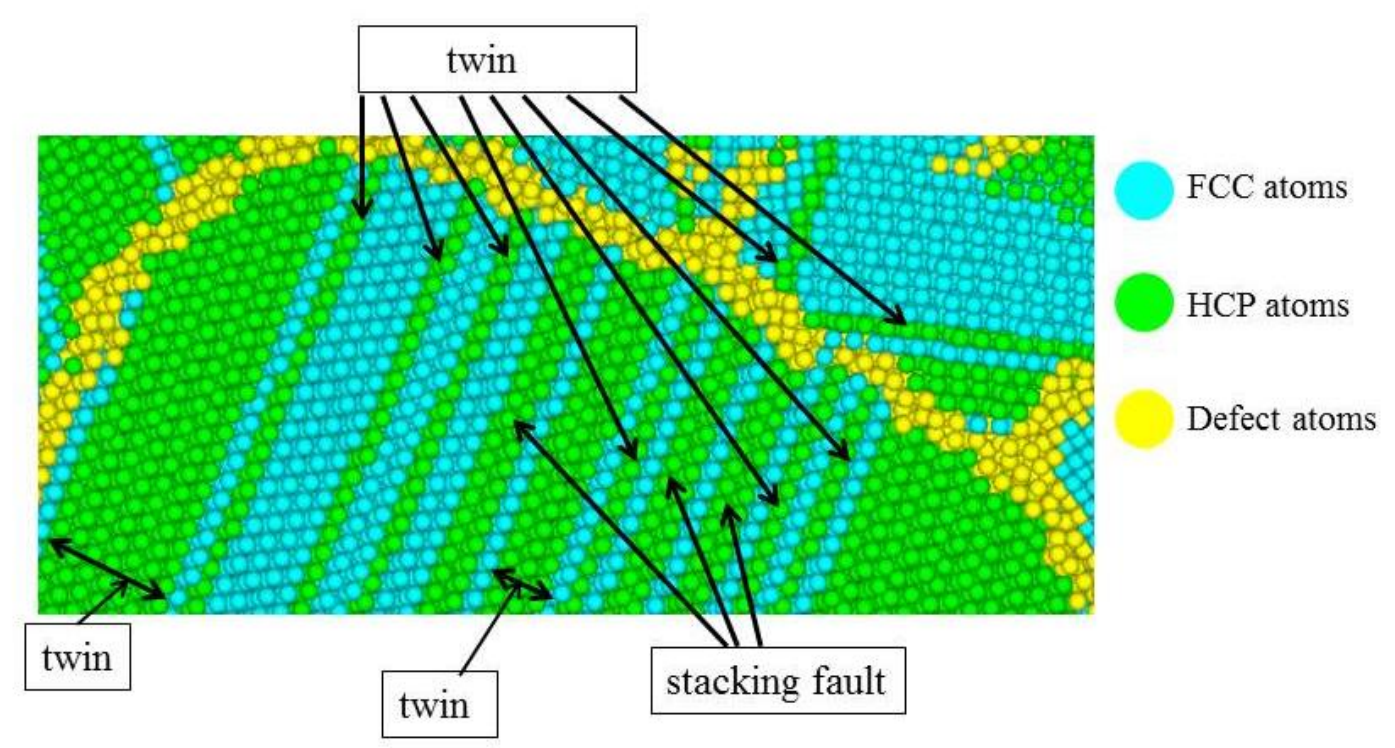

Figure 6.11: A small region of nano-crystalline structure generated in the simulation. Some of the twins and stacking faults are indicated in the figure.

Moreover, five-fold twinning has been observed in the nano-crystalline structure as shown in Fig. 6.12. In fact, the five-fold twinned structure has been experimentally observed in nanowires/nanoparticles of $\mathrm{Ag}$ [104,105], and other metals, such as $\mathrm{Cu}, \mathrm{Au}$, and Pd [106]. Theoretical models of their formation have also been discussed in [106]. Briefly, the possibility of the formation of five-fold twinning structure is related to the fact that the angle between two close-packed $\{111\}$ planes in the FCC lattice is $70.5^{\circ}$ as shown in Fig. 6.13. Since $70.5^{\circ} \times 5=352.5^{\circ} \sim 360^{\circ}$, the five-fold twinning structure can then be formed by stacking five FCC crystallites together with twin boundaries in between. To accommodate the difference between $352.5^{\circ}$ and $360^{\circ}$, the elastic deformation occurs when the size of this structure is small, on the order of nanometers as the ones obtained in our simulations. When the size is bigger, e.g. tens of nanometers, the high stresses can be relaxed through different channels [107-110], such as the formation of dislocations or a hollow centeral region. As we just discussed above, each FCC lattice corresponds to a tetrahedron. In principle, each edge of a tetrahedron can be a potential five-fold axis, by packing it with other tetrahedra. Therefore, more complicated structures with five-fold symmetry can be generated by packing the basic unit of tetrahedron, such as the structure of connected five-fold twinning as in Figs. 6.12b and 6.12c, the decahedron/icosahedron as shown in Fig. 6.14, and so on. 
(a)
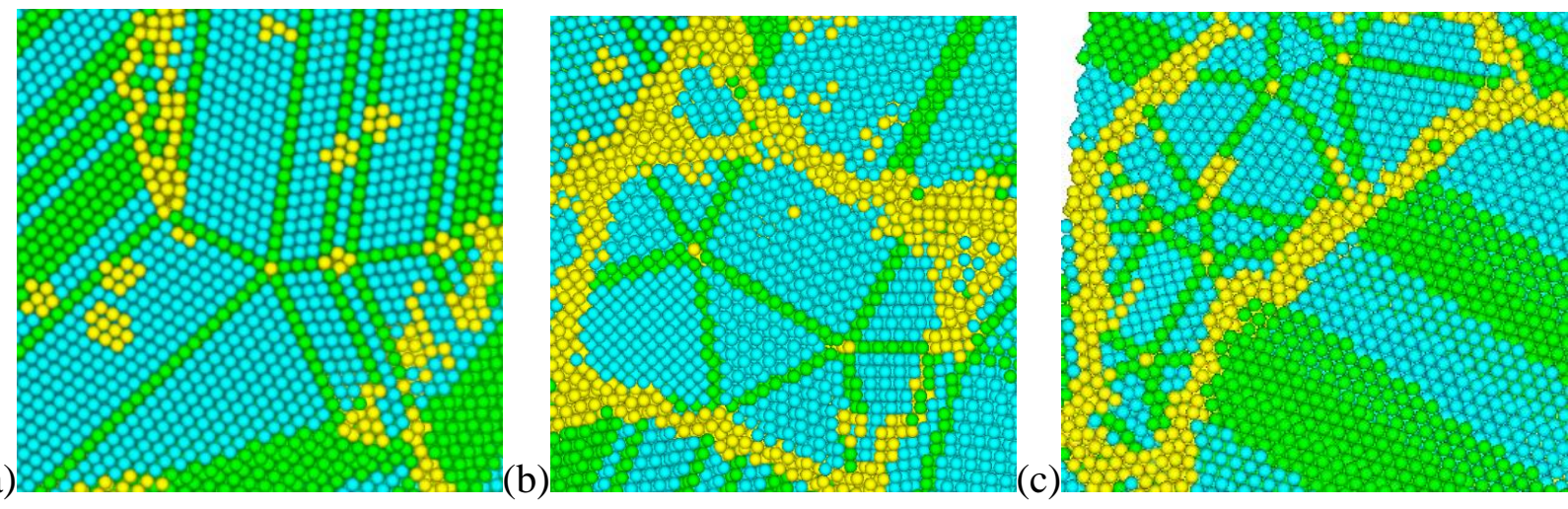

Figure 6.12: Three examples of five-fold twinning observed in the structure of laser-generated nano-crystalline surface region of the irradiated Ag (001) target. (a) shows an isolated 5-fold twinning structure. (b) and (c) show the structure of connected five-fold twinning.

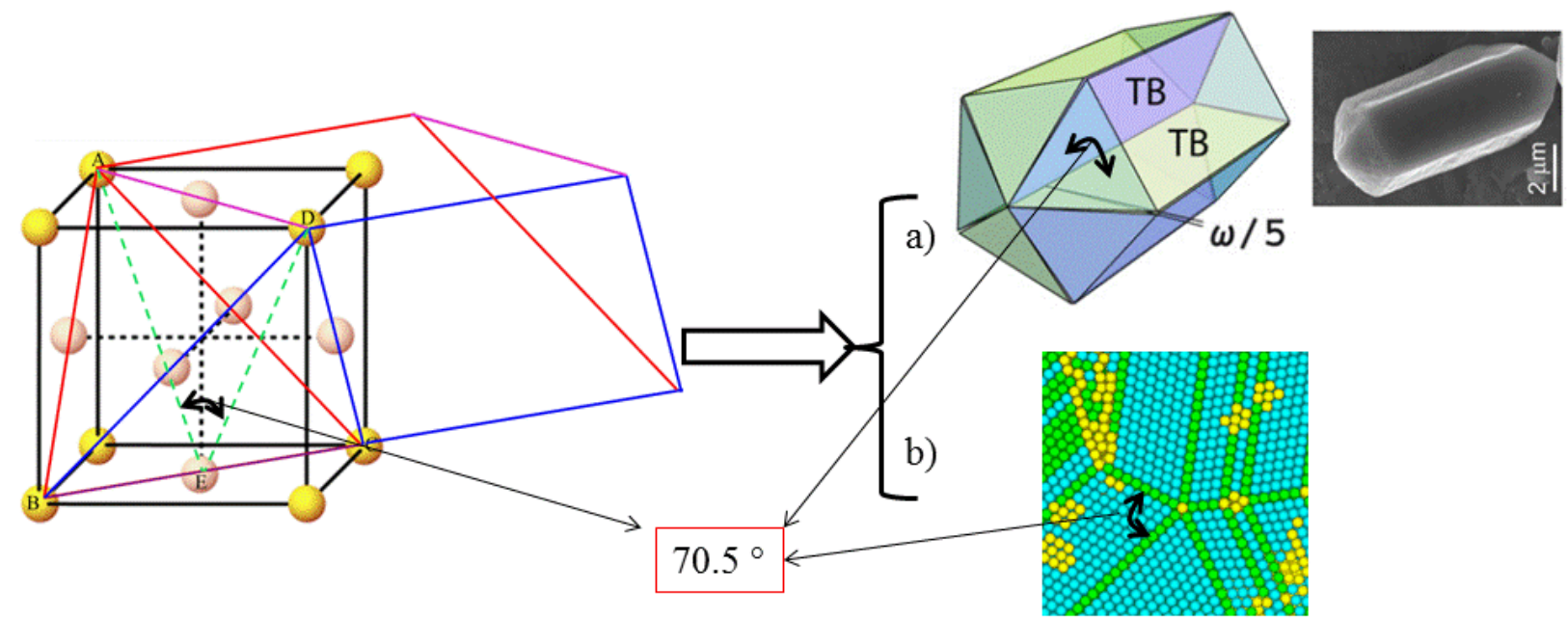

Figure 6.13: Formation of five-fold twinning structure: (a) micro(nano)-rods with five-fold symmetry adopted from Ref. [110], (b) nano-structure of five-fold symmetry obtained in our simulations. 

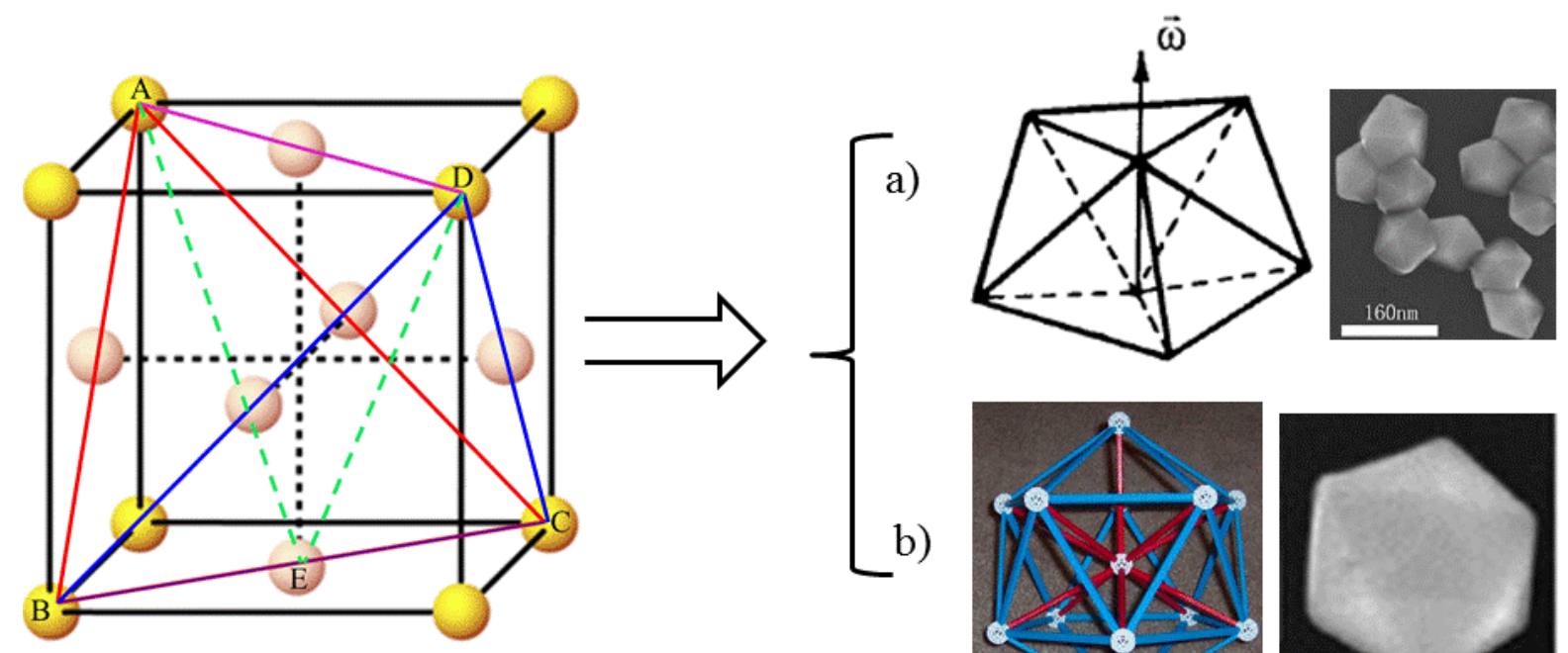

b)
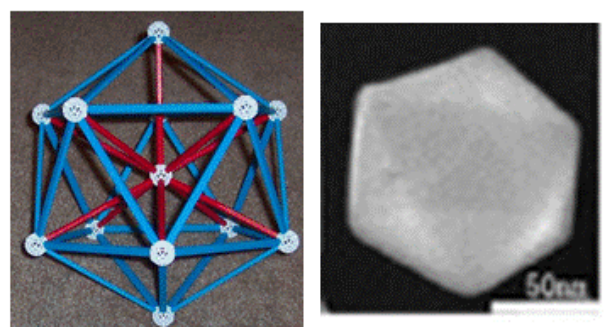

Figure 6.14: Formation of five-fold twinning structure. (a) Decahedron and a SEM image of Ag decahedral nanoparticles from Ref. [105], and (b) Icosahedron and a SEM image of Ag icosahedral nanoparticle from Ref. [105].

\subsection{Laser irradiation at $0.09 \mathrm{~J} / \mathrm{cm}^{2}$ : spallation and generation of a frozen nanospike}

During the laser irradiation at a fluence of $0.09 \mathrm{~J} / \mathrm{cm}^{2}$, the photomechanical spallation and formation of a thin and long nanospike on the surface of the target take place.

\subsubsection{Photomechanical spallation}

Fig. 6.15 illustrates the evolution of temperature, pressure, collective velocity, and density near laser-irradiated surface at laser fluence of $0.09 \mathrm{~J} / \mathrm{cm}^{2}$ up to $3.24 \mathrm{~ns}$. In comparison with the laser irradiation at $0.085 \mathrm{~J} / \mathrm{cm}^{2}$, the photomechanical effect here is stronger as indicated by the higher relative collective velocity of the top surface layer away from the substrate. For example, at $200 \mathrm{ps}$, the velocity of the top layer relative to the substrate is $\sim 1 \AA / \mathrm{ps}(\sim 100 \mathrm{~m} / \mathrm{s})$ at $0.09 \mathrm{~J} / \mathrm{cm}^{2}$, which is more than two times larger than $\sim 0.4 \AA / \mathrm{ps}(40 \mathrm{~m} / \mathrm{s})$ at $0.085 \mathrm{~J} / \mathrm{cm}^{2}$ as seen in the velocity contour plots of Figs. $6.1 \mathrm{~b}$ and $6.1 \mathrm{c}$. Induced by a stronger unloading tensile wave, the voids are formed, grow and coalesce. At $\sim 600 \mathrm{ps,} \mathrm{a} \mathrm{gap} \mathrm{with} \mathrm{material} \mathrm{density} \mathrm{less}$ than $10 \%$ of the initial density before the irradiation appeara as seen in Fig. 6.15. Even though the areas with the material density less than $10 \%$ are blanked in the contour plots shown in Fig. 6.15 , the top surface layer is still linked to the bottom substrate by a bridge of liquid materials up to the time of $\sim 3 \mathrm{~ns}$. The motion of the top layer drives void growth and bridge elongation, leading to the final separation of the top surface layer at $\sim 3 \mathrm{~ns}$ as seen in Fig. 6.16. After the 
separation, a thin nanospike with diameter of $\sim 6 \mathrm{~nm}$ is formed due to the breakdown of the bridge. Under the condition of strong undercooling, the nanospike rapidly freezes up by homogeneous resolidification as seen in Fig. 6.17.

(a)

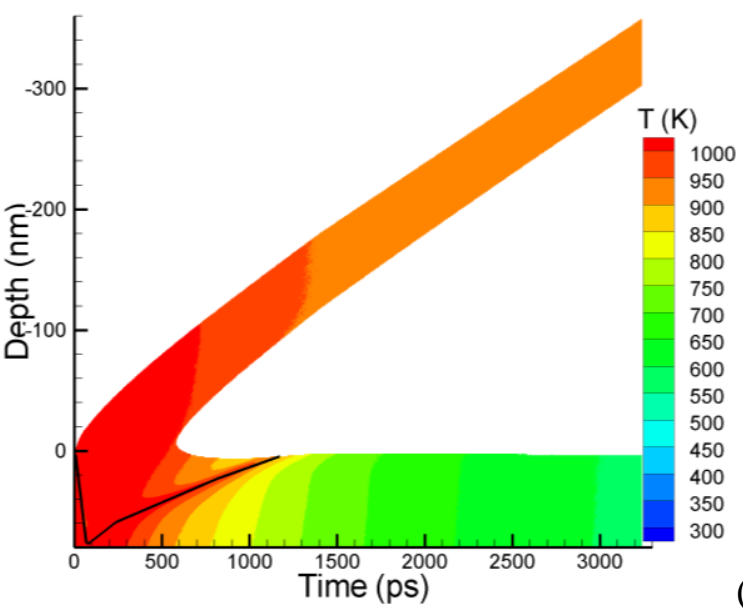

(c)

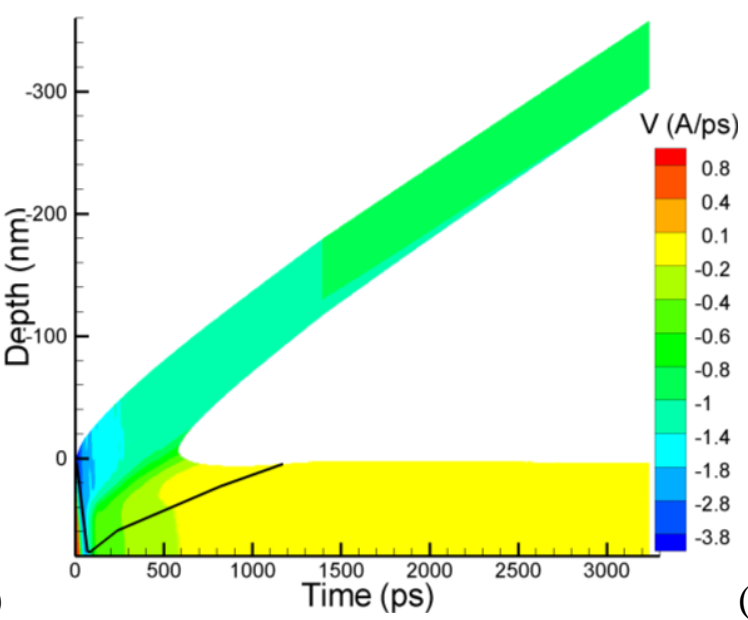

(b)
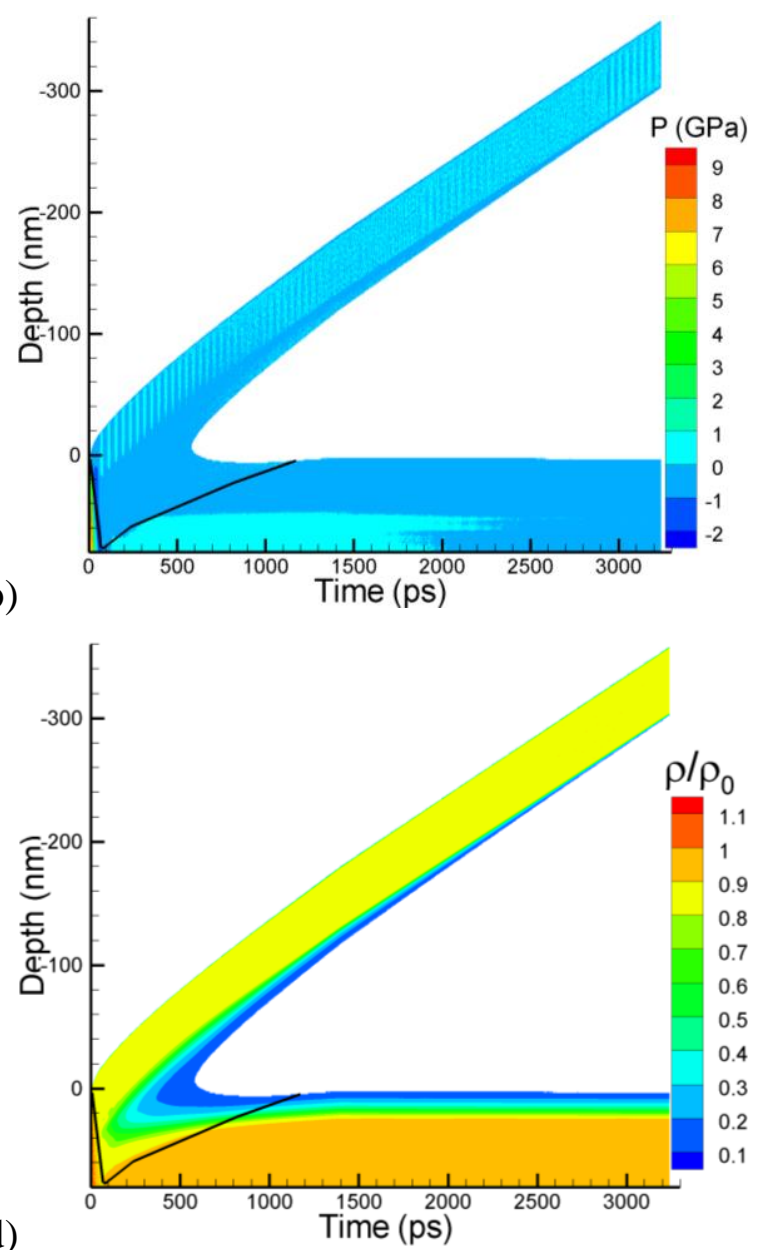

Figure 6.15: Contour plots showing the evolution of (a) the lattice temperature, (b) pressure, (c) collective velocity in the direction normal to the surface and (c) density normalized to the density of $\mathrm{Ag}$ at $300 \mathrm{~K}$ before the irradiation as a function of time and depth in the surface region $(<80$ $\mathrm{nm}$ ) for the simulation of $100 \mathrm{fs}$ short pulse laser irradiation on $\mathrm{Ag}(001)$ target at a fluence of $0.09 \mathrm{~J} / \mathrm{cm}^{2}$. Areas where the density of the material is less than $10 \%$ of the initial density before the irradiation are not shown in the plots. The black lines separate the "melted regions" from the crystalline bulk of the target. 


\begin{tabular}{llllll}
\hline $0.1 \mathrm{~ns}$ & $0.2 \mathrm{~ns}$ & $0.3 \mathrm{~ns}$ & $0.4 \mathrm{~ns}$ & $0.6 \mathrm{~ns}$ & $0.8 \mathrm{~ns}$
\end{tabular}
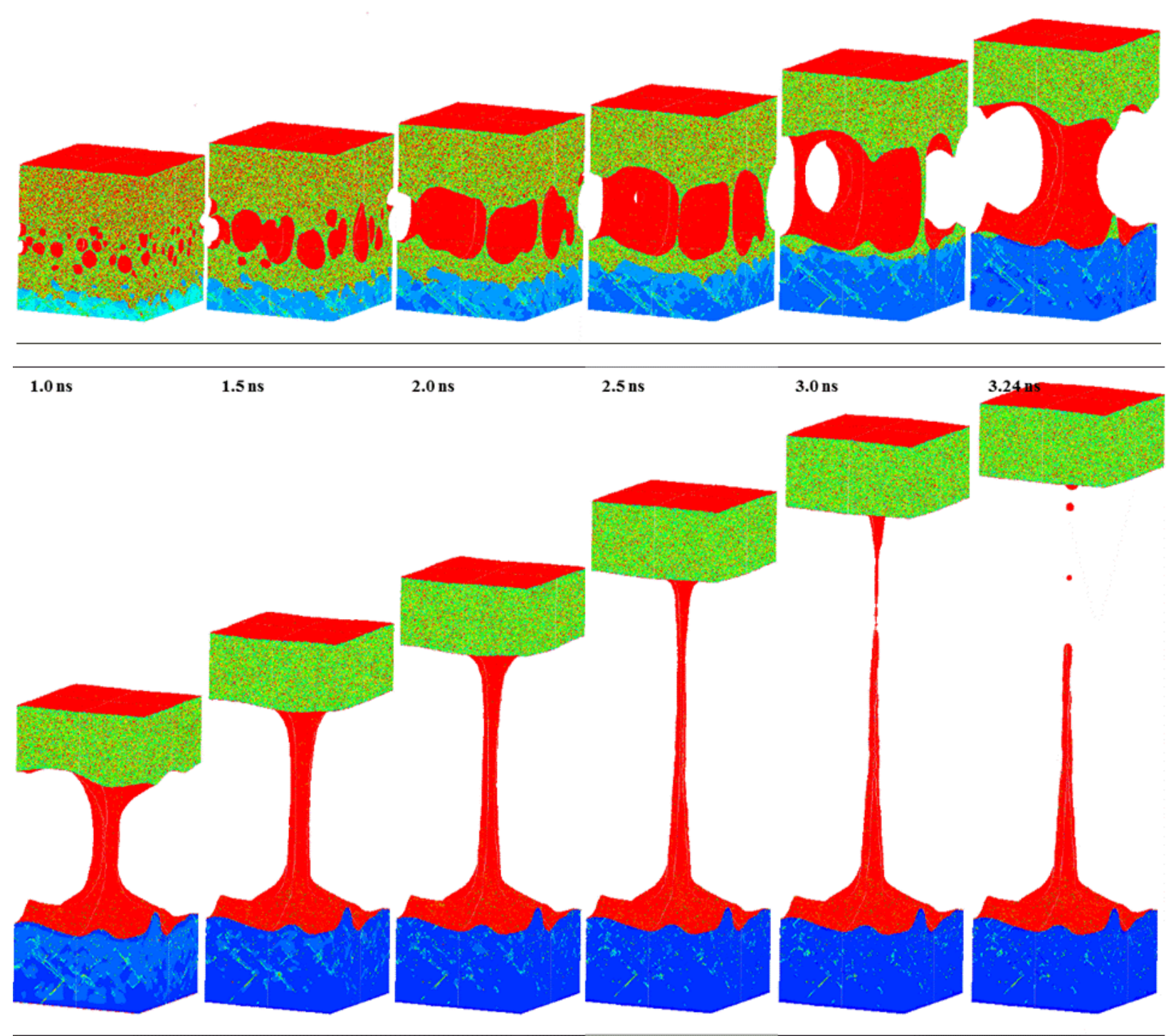

Figure 6.16: A series of snapshots for the simulation performed at a laser fluence of $0.09 \mathrm{~J} / \mathrm{cm}^{2}$. The void growth, bridge elongation and the final separation of the top surface layer are clearly seen. 


\begin{tabular}{llllll}
\hline $0.1 \mathrm{~ns}$ & $0.2 \mathrm{~ns}$ & $0.3 \mathrm{~ns}$ & $0.4 \mathrm{~ns}$ & $0.6 \mathrm{~ns}$ & $0.8 \mathrm{~ns}$
\end{tabular}
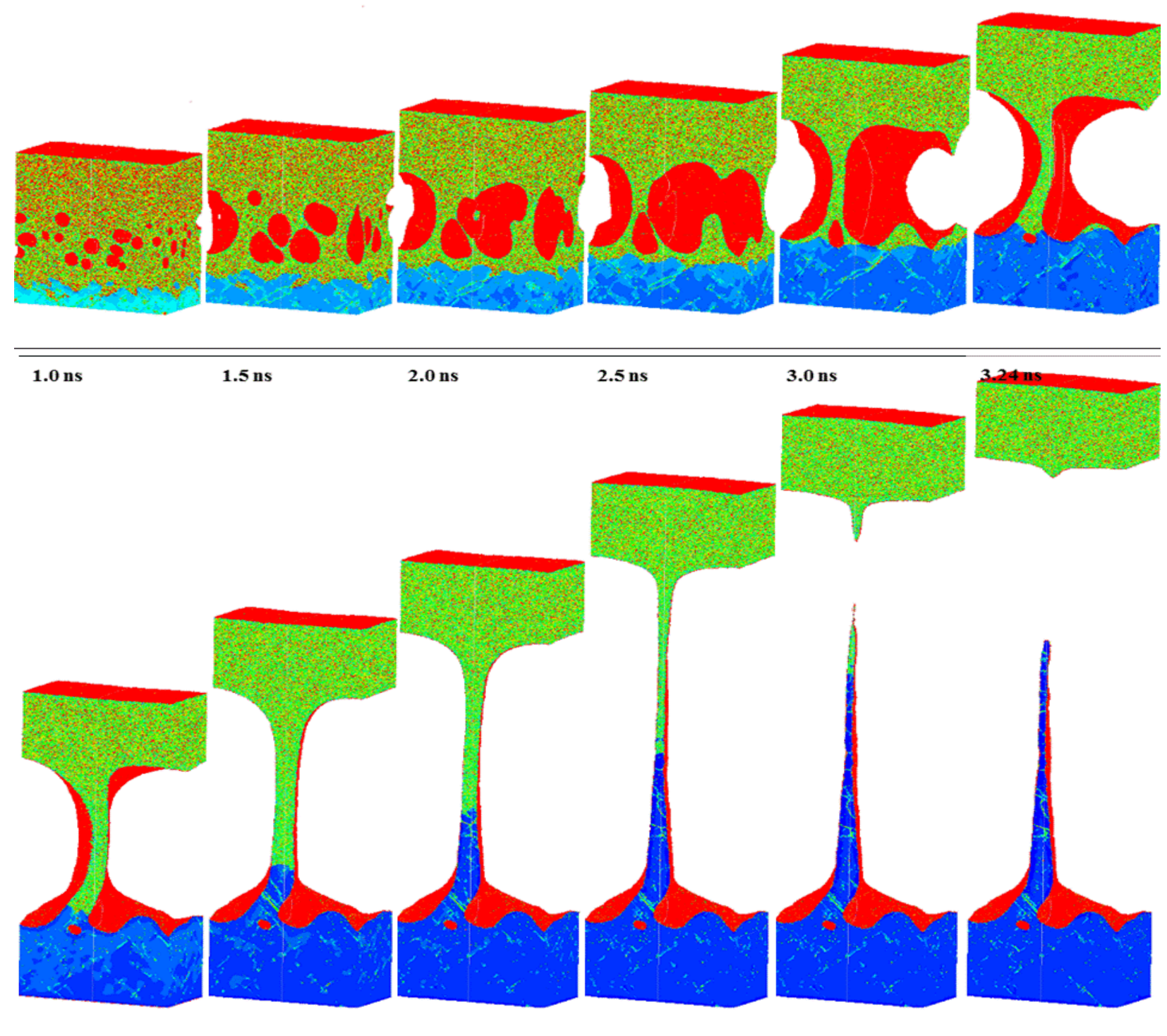

Figure 6.17: Cross sections through the middle of the computational cell of the atomic configurations shown in Fig. 6.16. The cross sections are shown to illustrate the resolidification process, especially the one in the nanospike. 


\subsubsection{Structure of the nanospike}

As seen in Fig. 6.18, the strong undercooling triggers homogeneous nucleation and growth (see Fig. 6.18a) of randomly oriented crystalline regions in the elongated bridge during the spallation process (see Figs. 6.16, 6.17, and 6.18b). The resolidification process can be clearly seen from the temperature contour plot in Fig. 6.18a: due to the exothermic nature of resolidification, the homogeneous crystal nucleation can be identified by the temperature increase at local sites marked by cross signs in Fig. 6.18a. Following the homogeneous nucleation, multiple crystallites grow and meet each other as indicated by the spread of the areas of the elevated temperature marked by black dashed arrows in Fig. 6.18a. The homogeneous nucleation and growth of multiple crystallites results in the formation of a thin and long nanospike with nanocrystalline structure after the spallation of a liquid layer. The nanocrystalline structure of the nanospike is clearly seen in Fig. 6.18c: nanograins have closepacked structure (FCC/HCP) and random crystallographic orientation.

(a)

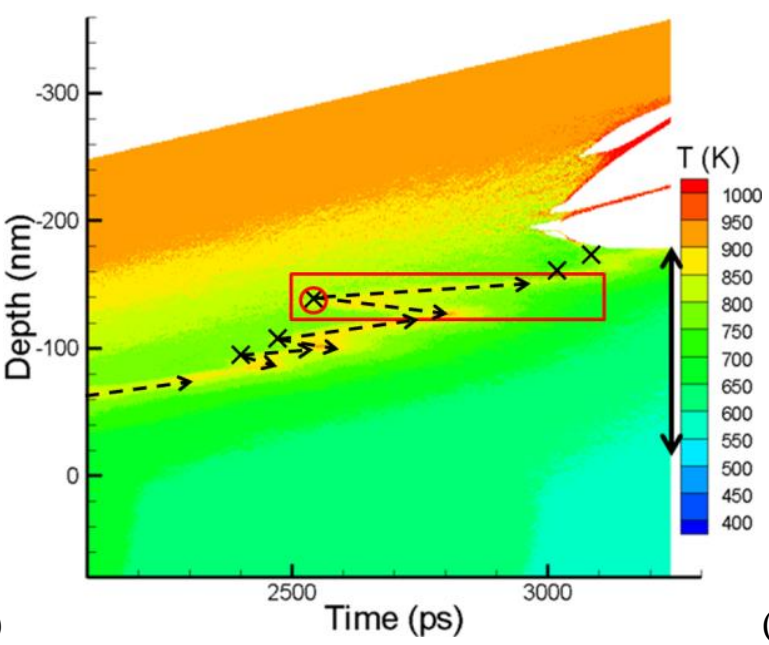

(b)

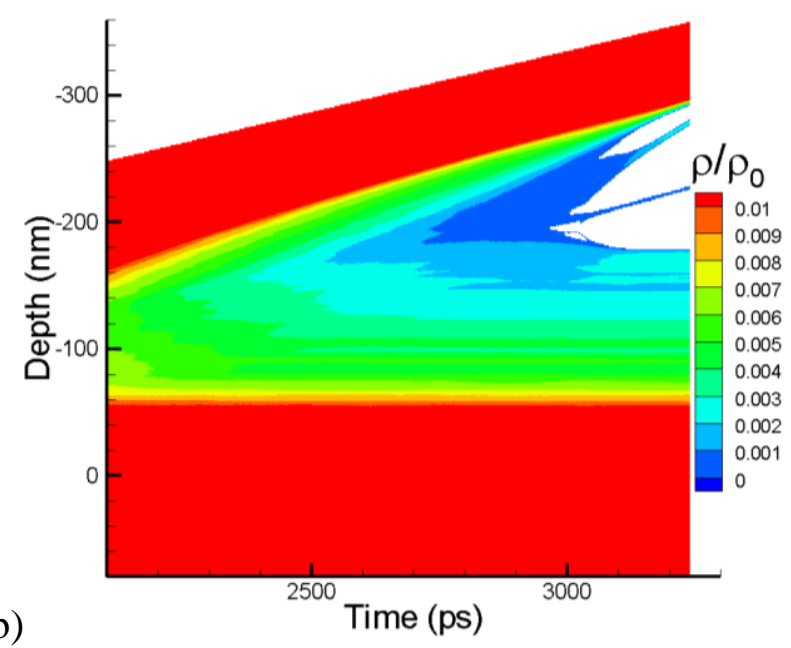

c)

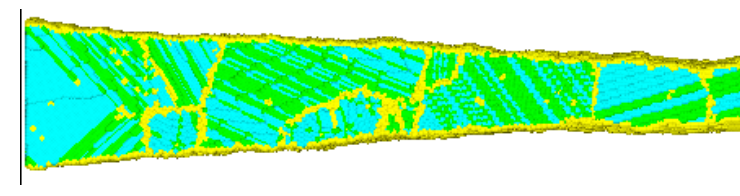

Figure 6.18: Contour plots of temperature (a) and density (b) focused on the region of the solidification of the elongated bridge. Some obvious homogeneous nucleation sites are marked in (a) by the cross signs and their growth shown by the black dashed arrows. A cross section through the middle of the nanospike with atoms colored by its local structural environment (the coloring convention is the same as in Figs. 6.8a and 6.8b is shown in (c). 
Interestingly, the five-fold twinning structure is also found to be formed in the nanospike. The nucleation of this five-fold twinning structure is illustrated in Fig. 6.19. First, a small FCC crystallite is nucleated. Then two twin boundaries are formed due to the irregular stacking. New FCC lattices with different orientations grow from the twin boundaries. Then new twin boundaries are formed again, from which new FCC crystallites grow. Finally, the five-fold twinning structure is formed when five FCC crystallites with different orientations pack together as shown in Fig. 6.19.

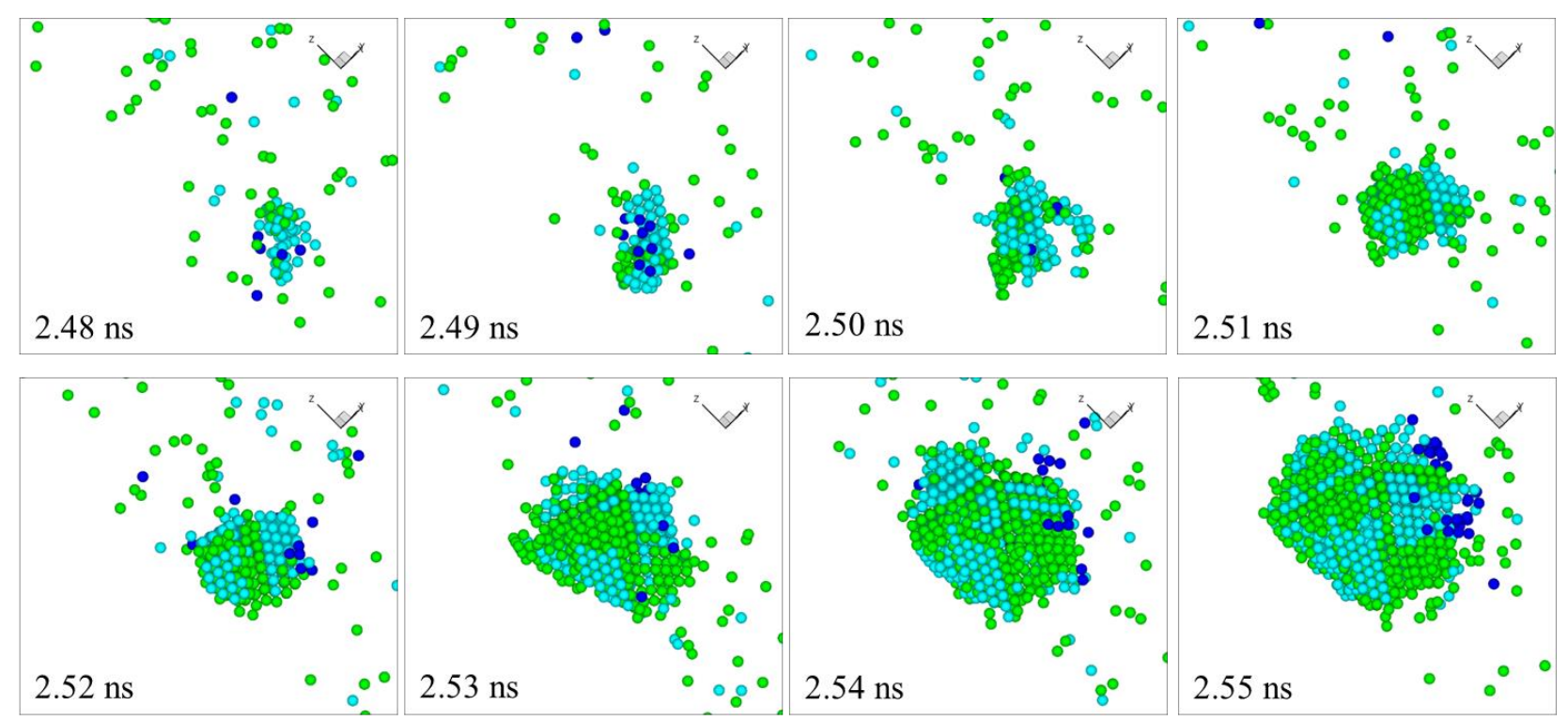

Figure 6.19: Nucleation of five-fold twinning structure in the region outlined by the red circle in Fig. 6.18a. Atoms are colored by their local structural environments and the coloring is the same as in Fig. 6.8a. The "defect" (liquid) atoms are blanked to clearly illustrate the nucleation process.

By following the FCC-twin-FCC cycle, a very complicated five-fold twinning structure is formed in the nanospike as seen in Fig. 6.20. More detailed analysis has demonstrated that the this complex five-fold twinning structure can be represented by 6 inter-penetrating icosahedra truncated by the free surface of the nanospike as seen in Fig. 6.21. 


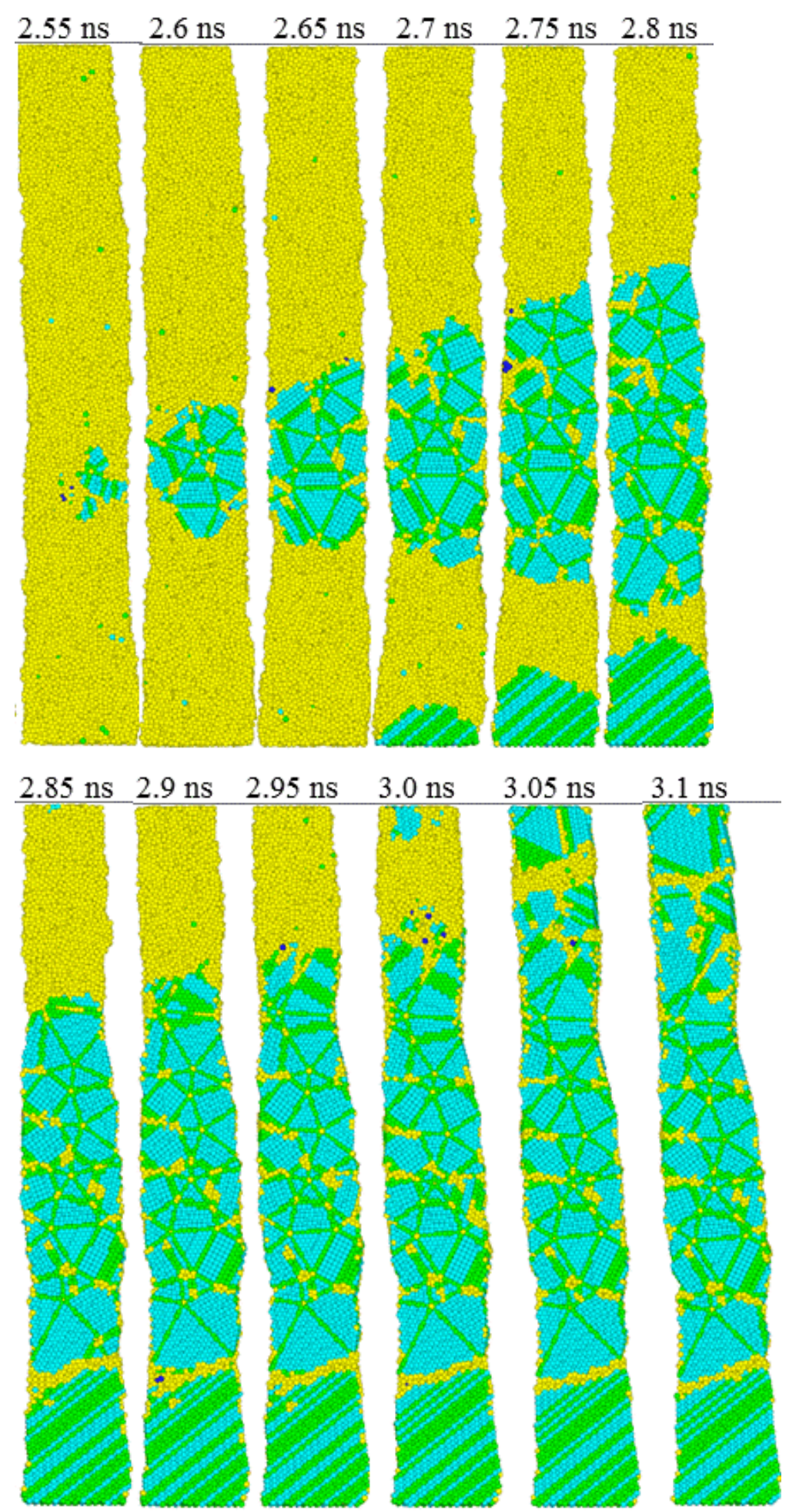

Figure 6.20: A series of cross sections of the nanospike made to illustrate the growth of five-fold twinning structure in the region outlined by the red rectangle in Fig. 6.18a. Atoms are colored by their local structural environments and the same coloring convention and scale as in Fig. 6.8a are used. Surface atoms are also blanked in the figures. 
a)

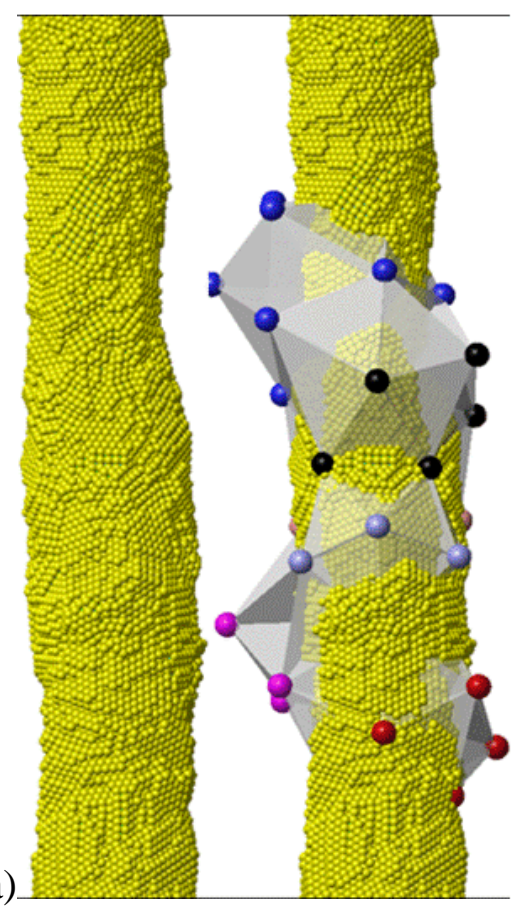

b)
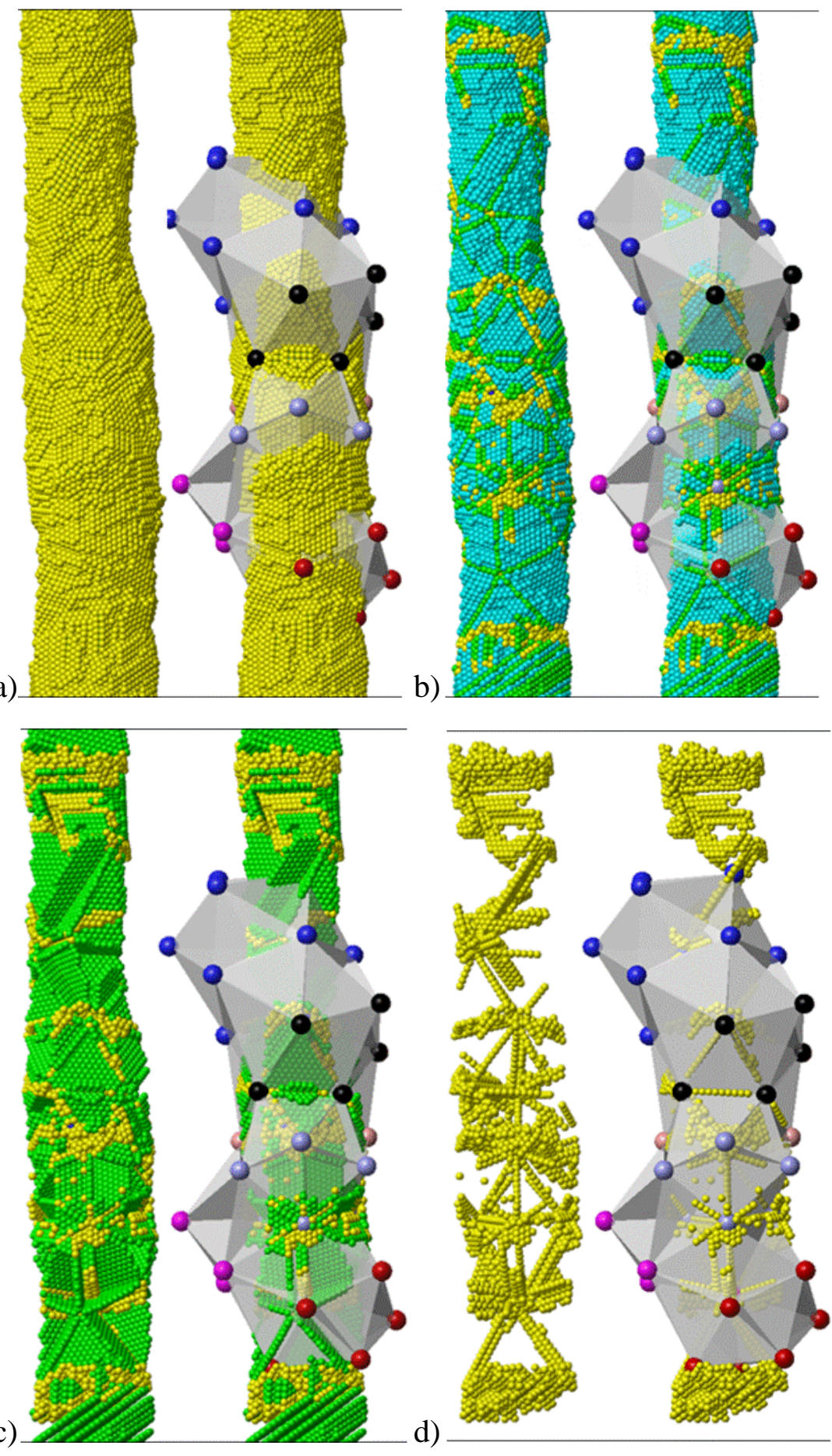

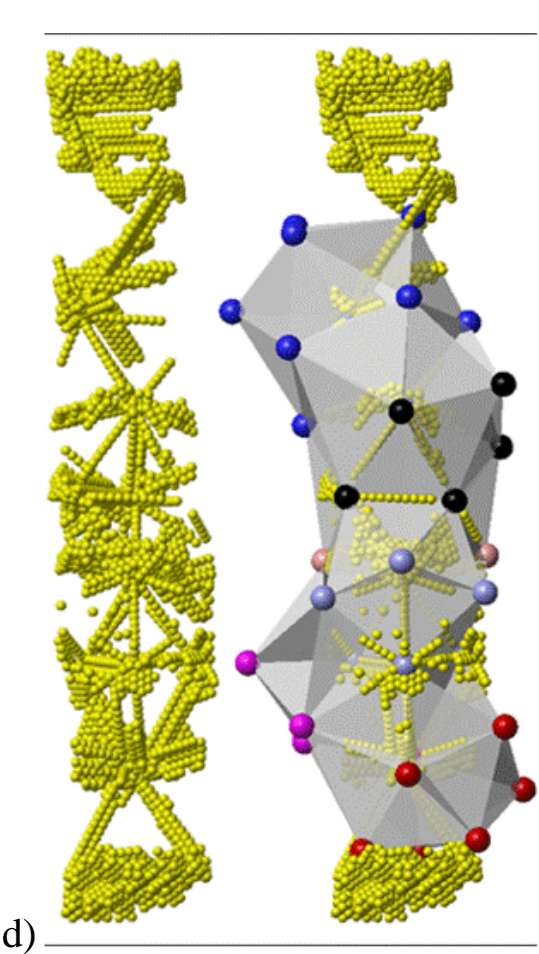

Figure 6.21: (a) A snapshot to illustrate the five-fold twinning structure: 6 inter-penetrating icosahedra truncated by the free surface of the nanospike. To clearly see the structure, surface atoms are blanked in (b), both surface atoms and FCC atoms are blanked in (c), and surface atoms, FCC atoms and HCP atoms are blanked in (d). All are the same snapshots with atoms colored by its local structural environment. 


\subsection{Discussions}

\subsubsection{Surface swelling}

The large scale simulations further demonstrate the void formation and spallation due to the short pulse laser-induced photomechanical processes. In the case of $100 \mathrm{fs}$ laser irradiation of $\mathrm{Ag}$ (001) bulk target, the threshold absorbed fluence for voids formation is found to be in the range between $0.08 \mathrm{~J} / \mathrm{cm}^{2}$ and $0.085 \mathrm{~J} / \mathrm{cm}^{2}$, while the spallation threshold is between $0.085 \mathrm{~J} / \mathrm{cm}^{2}$ and $0.09 \mathrm{~J} / \mathrm{cm}^{2}$. During laser irradiation at the fluence above the spallation threshold, e.g., 0.09 $\mathrm{J} / \mathrm{cm}^{2}$, the growth and coalescence of voids lead to the formation and eventual break down of an elongated bridge between the substrate and the top liquid layer (see Figs. 6.16 and 6.17). The spallation can be explained by the fact that the expansion of the bridge hardly decelerates the layer that moves away from the target with a velocity of about $100 \mathrm{~m} / \mathrm{s}$ (see Fig. 6.15c). In contrast, laser irradiation at the fluence above the voids formation threshold but below the spallation threshold, e.g., $0.085 \mathrm{~J} / \mathrm{cm}^{2}$, creates the conditions for voids to be captured by the resolidification front. The captured voids increase the volume of the surface region, leading to an effective "swelling" of the irradiated target, by about $17 \mathrm{~nm}$ at a fluence of $0.085 \mathrm{~J} / \mathrm{cm}^{2}$. The computational prediction of the generation of porous structure of a surface region of the irradiated target provides a hint for explaining the recently reported experimental observation of surface swelling, or "frustrated ablation," in Al and Ag targets irradiated by 100 fs laser pulses $[19,20]$.

The small gap between the voids formation threshold and the spallation threshold can be explained by the use of periodic boundary conditions and uniform laser energy density in the lateral directions used in the simulations, which facilitates percolation of voids in the lateral directions and separation of the liquid layer from the target. In experiments, on the contrary, a non-uniform laser energy deposition within the laser spot and a restraining effect of the cold periphery of the laser spot on the separation and ejection of the liquid layer accelerated during the initial relaxation of the laser induced stresses are likely to suppress the onset of spallation and to extend the range of laser fluences where the effect of surface swelling is observed. This has been verified in experiments, where the material removal starts to occurs at a higher absorbed fluence of $0.10 \mathrm{~J} / \mathrm{cm}^{2}$ (incident fluence of $3.17 \mathrm{~J} / \mathrm{cm}^{2}$ ), as compared to the spallation threshold of $0.09 \mathrm{~J} / \mathrm{cm}^{2}$ predicted in the simulations. If we assume that the laser spot has a radius of $15 \mu \mathrm{m}$ 
(Full Width at Half Maximum, FWHM), and the laser fluence decays radially with a Gaussian spatial profile, the simulations at $0.08 \mathrm{~J} / \mathrm{cm}^{2}, 0.085 \mathrm{~J} / \mathrm{cm}^{2}$, and $0.09 \mathrm{~J} / \mathrm{cm}^{2}$ can be mapped to the experimental irradiation conditions at the incident fluence $3.17 \mathrm{~J} / \mathrm{cm}^{2}$ as shown in Fig. 6.22. No swelling at $0.08 \mathrm{~J} / \mathrm{cm}^{2}$ is verified in experiment. No obvious swelling at $0.085 \mathrm{~J} / \mathrm{cm}^{2}$ is probably due to the fact that the swelling is too small $(\sim 17 \mathrm{~nm})$ to be observed in experiment. The surface swelling at $0.09 \mathrm{~J} / \mathrm{cm}^{2}$ is as expected. The material removal at the top swollen part is reasonable, as the local fluence in the center of the laser spot is more than $\sim 11 \%$ higher than the spallation threshold. The proposed mechanism is as shown in Fig. 6.22c.

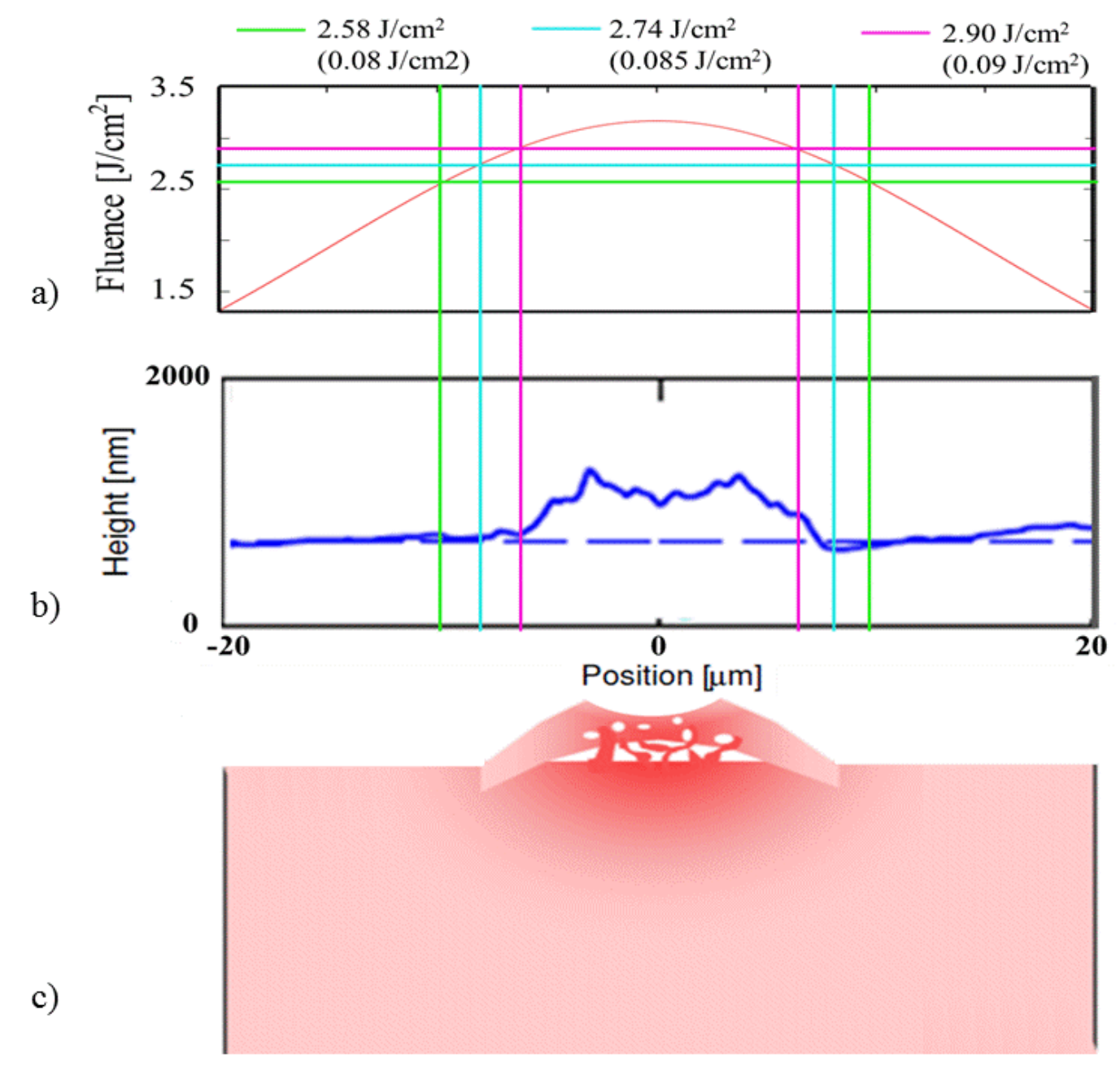

Figure 6.22: Mapping the results of the simulations to the irradiation conditions of the experiment at the incident fluence of $3.17 \mathrm{~J} / \mathrm{cm}^{2}$ with the assumption that the laser energy deposition has a Gaussian energy profile and FWHM of $15 \mu \mathrm{m}$. (a) Laser fluence profile, (b) AFM line outs after laser irradiation, and (c) the proposed swelling mechanism. 


\subsubsection{Microstructure}

Besides the surface swelling, the large-scale simulations directly demonstrate the generation of nanocrystalline structure in a surface layer or nanoscale morphological surface features (e.g., nanospike) by the short pulse laser irradiation. Fast energy deposition during the short pulse laser irradiation leads to a rapid heating and melting, followed by a very fast cooling $\left(10^{11}-10^{12} \mathrm{~K} / \mathrm{s}\right)$ that can hardly be achieved by any other means. The surface layer can cool down to a temperature far below the equilibrium melting temperature (e.g., $0.69 \mathrm{~T}_{\mathrm{m}}$ in the simulation of $\mathrm{Ag}$ (001) target) before the arrival of heterogeneous resolidification front. Under such strong undercooling, the homogeneous nucleation and growth of multiple randomly oriented crystallites results in a rapid solidification and generation of nanocrystalline structure of the top layer of the target. In the case of $\mathrm{Ag}$, all nanograins are found to have close-packed structure, with large number of stacking faults and twin boundaries. More interestingly, the complicated five-fold twinning structure is formed. The grain boundaries, stacking faults, and twin boundaries are all likely to present strong barriers for dislocation propagation, resulting in the effective hardening of the generated nanocrystalline structure (indentation simulations are planned to test the hardness of the nanocrystalline layer).

\subsubsection{Complex surface morphology}

As seen in the simulation of laser irradiation on $\mathrm{Ag}$ at $0.09 \mathrm{~J} / \mathrm{cm}^{2}$, which is just above spallation threshold, a thin and long nanospike is formed after spallation. As the laser fluence increases above the spallation threshold, the size of the region affected by the void formation is increasing and the thickness of the ejected liquid layer is decreasing. These trends are illustrated in Fig. 6.23, where snapshots are shown for a simulation performed for an Al target at a laser fluence that is about $25 \%$ above the spallation threshold. The appearance, growth, and coalescence of voids in this case proceeds at a higher temperature and leads to the formation of a complex structure of interconnected liquid regions. The top liquid layer separates from the target by 500 ps and moves away with a velocity of about $600 \mathrm{~m} / \mathrm{s}$. Although this simulation has not been continued until the complete resolidification, we can speculate that the competition between the solidification of the surface region and a relatively slow collapse/flattening of the foamy liquid structure generated in the course of spallation may be responsible for the formation of 
complex nanoscale surface morphology commonly observed in femtosecond laser processing experiments, e.g. [111-114].
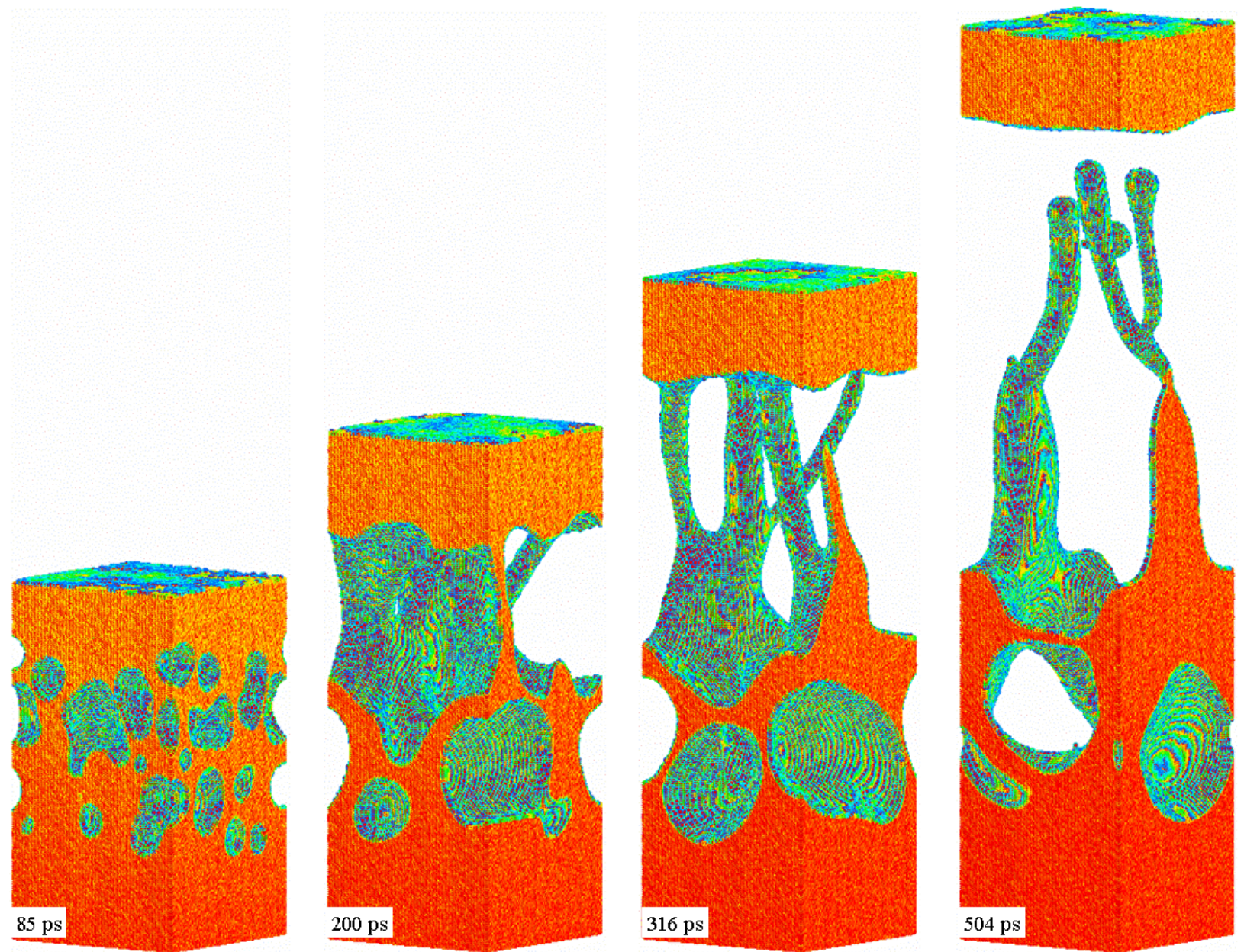

Figure 6.23: Snapshots of atomic configurations generated in TTM-MD simulations of bulk Al irradiated by $100 \mathrm{fs}$ laser pulses at absorbed fluence $0.09 \mathrm{~J} / \mathrm{cm}^{2}$. Only top parts of the computational cells are shown in the snapshots. The atoms are colored by local density of their immediate surroundings. 
PART II: Short Pulse Laser-induced Photoacoustic Control of Surface Diffusion (In collaboration with Prof. Vladimir Yu. Zaitsev from Institute of Applied Physics of Russian Academy of Science) 


\section{Introduction}

Short pulse laser irradiation of a strongly absorbing target can produce strong stresses in the region of the laser energy deposition and result in the generation of acoustic pulses of a very high intensity and frequency in the range of up to hundreds of GHz [115]. The generation of the photoacoustic pulses is defined by the condition of stress confinement [96]. Photoacoustic pulses produced via the short pulse laser irradiation were first observed in 1963 [21]. Due to the periodic boundary condition and the uniform laser energy deposition used in simulations, only bulk acoustic waves are generated and observed in the pressure contour plots illustrating the simulations discussed in Part I. In experiments, acoustic propagation is more complex and both bulk and surface acoustic waves can be generated [22,23,116-118]. As the laser technology progresses, the laser unltrasonic techniques develop as well and are demonstrated to be an efficient tool in many areas. For example, the laser-induced acoustic waves are used for nondestructive evaluation and material characterization, such as measurements of material elastic constants, density, film thickness and characterization of microstructure, e.g. [24,25,119,120]. The ability of the acoustic waves to desorb molecules, atoms and ions from surfaces has also been discussed in literature and supported by experimental evidence and theoretical calculations [16-18]. In dry laser cleaning, laser generated transient surface deformation is used for removal of small particles from the irradiated surfaces [11-14].

In 1980, K. A. Nelson et al. [121] proposed a transient grating method, as shown in Fig. 7.1a, of creating surface acoustic waves (SAWs) through the interference between the incoming excitation laser pulses. The interference between the incoming excitation laser pulses results in an oscillatory density of excited states. The period of the interference pattern defines the SAWs wavelength, which is limited in the range of optical wavelengths. The shortest acoustic wavelength generated to date using the transient grating method is approximately $750 \mathrm{~nm}$, with acoustic frequencies approaching $5 \mathrm{GHz}[122,123]$. To overcome the optical limit, an alternative approach [124], as shown in Fig. 7.1b, is developed by patterning a nanostructure on the surface. This pattern is then optically excited, generating local stress in the surface and thus exciting a SAW with a wavelength defined by the period of the patterned nanostructure. The shortest acoustic wavelength generated to date using this method of photoacoustic excitation of patterned surface is approximately $100 \mathrm{~nm}$, with acoustic frequencies approaching $100 \mathrm{GHz}[124,125]$. 
(a)

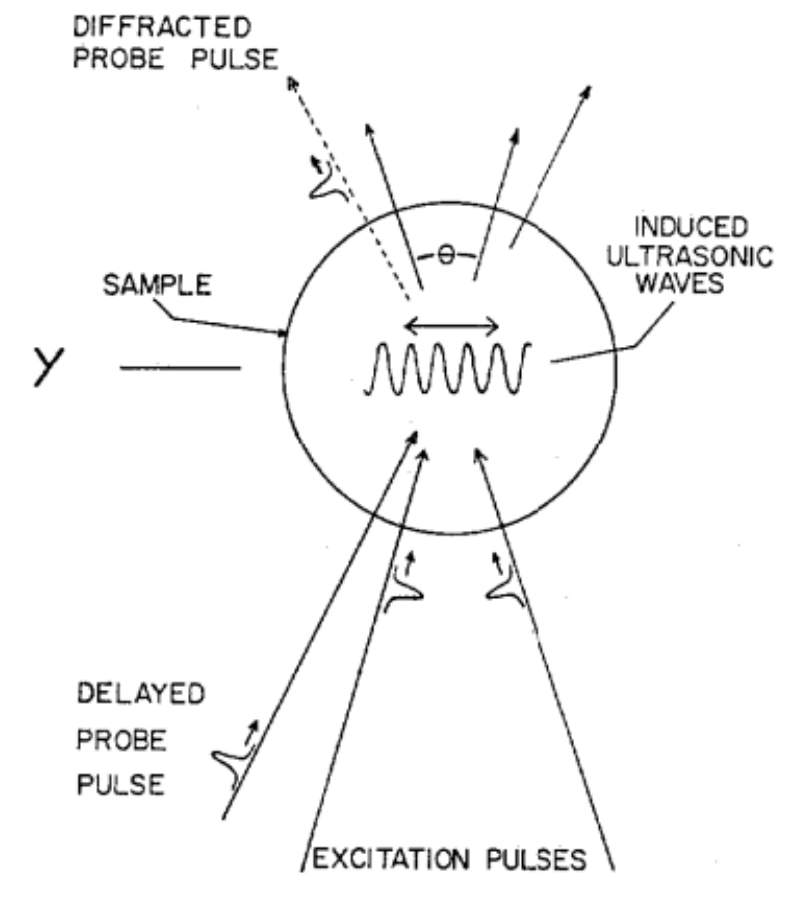

(b)

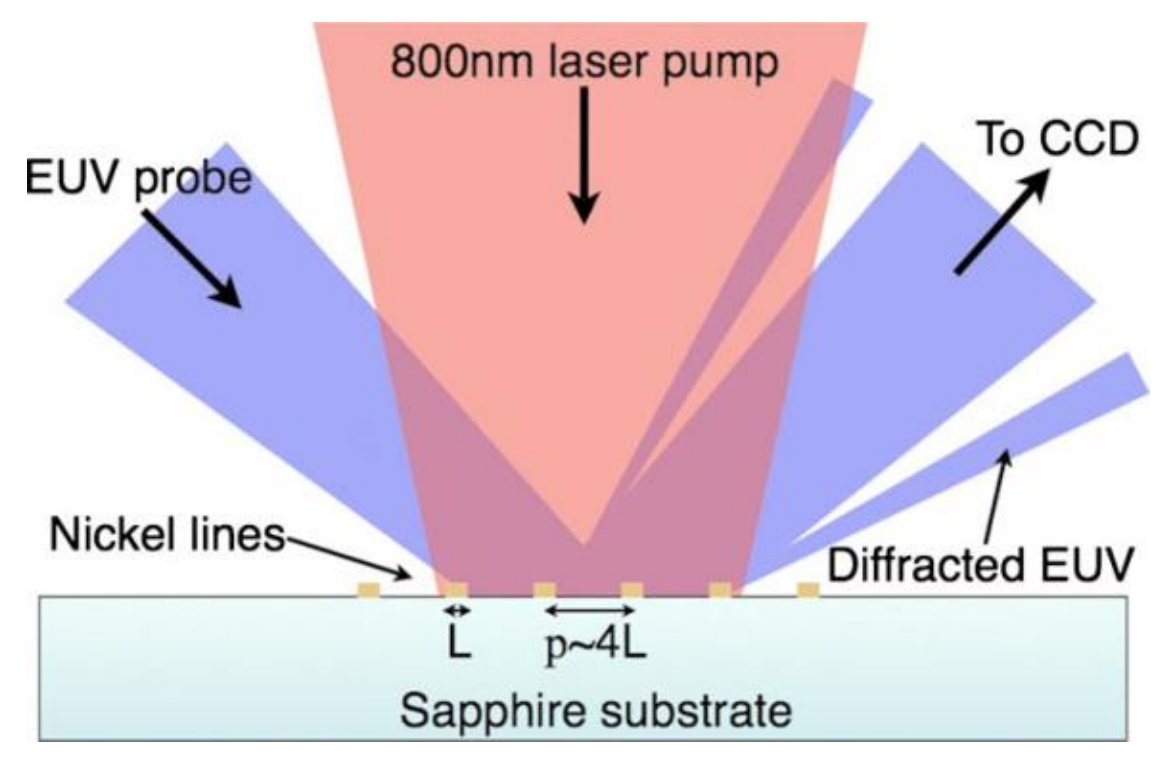

Figure 7.1: (a) Schematic illustration of the transient grating method to create SAWs. Interference between the incoming excitation pulses results in a periodic oscillatory density of excited states, that excites a SAW propagating in $\mathrm{z}$ diretion. This figure is adopted from Ref. [121]. (b) Schematic illustration of the method of photoacoustic excitation of patterned surface to create SAWs. Nickel lines of width $L$ and period $p$ are patterned on a sapphire substrate. An 800 laser pulse heats the Ni creating a periodic stress that excites a SAW. This figure is adopted from Ref. [124]. 
In this part of the thesis, we intend to explore the effect of the laser-induced surface acoustic waves (LISAWs) on surface diffusion of adsorbed species, as schematically shown in Fig. 7.2. The research questions to be addressed are briefly outlined below.

1). Is it possible to achieve a substantial increase in the surface mobility of physisorbed molecules by exposing the surface to running acoustic waves generated by short laser pulses?

2). Is it possible to achieve surface self-structuring (spatial modulation of adatom concentration) by exposing the substrate surface to a standing acoustic wave generated by short pulse laser pulses?

The first question is addressed theoretically and in a series of atomic-level MD simulations performed for a generic material system with interatomic interactions described by Lennard-Jones potential. The use of the simple interatomic potentials enables a computationallyefficient systematic study of the dependence of the surface processes on the parameters of the photoacoustic excitation and the characteristics of adsorbate molecules. It is found that a substantial diffusion enhancement (up to tens and hundreds of percents) by surface acoustic waves is possible. The mechanisms of acoustic enhancement of surface diffusion are explored and may provide new ideas for guiding the surface processes in film growth by photoacoustic stimulus. The results of the simulations are presented in section 8 .

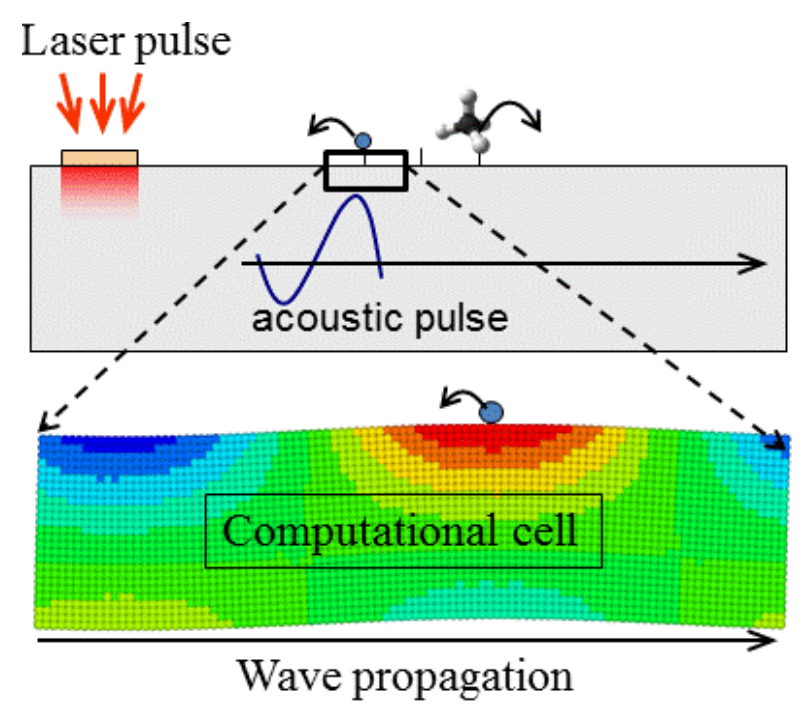

Figure 7.2: Schematic representation of the computational system used in MD simulations of adatom diffusion on a substrate in the presence of a LISAW propagating along the $x$ direction. More details can be found in section 8. 
The second question is first addressed theoretically and in a series of kinetic Monte Carlo (kMC) simulations. It is found that, in addition to the diffusion enhancement, the surface selfstructuring (spatial modulation of adatom concentration) can be effectively induced by standing SAWs. Due to the strong concentration dependence of the nucleation rate, one can expect that the ability of the SAWs to induce the spatial fluctuations of concentration can be used to control nucleation during the thin film deposition, and thus affect structural characteristics of the growing films. The results of self-structuring are presented in section 9. MD simulations for a generic material system with interatomic interactions described by Lennard-Jones potential is planned for the near future. These MD simulations are designed to verify the predictions of the theoretical analysis and $\mathrm{kMC}$ simulations and to provide additional insights into the role of dynamic coupling of SAWs to the adsorbates in surface structuring. 


\section{Acoustic enhancement of surface diffusion}

\subsection{Introduction}

The ability of surface acoustic waves (SAW), or Rayleigh waves, to propagate and transfer energy along a thin (several wavelengths deep) surface layer of substrate with little dissipation has been utilized in a number of applications ranging from nondestructive evaluation of mechanical properties and surface defects [126-128] to micro- and nano-scale manipulation of particles, biomolecules and fluid flow in microfluidics devices[129,130]. At the atomic level, however, the ability of SAW to directly affect surface mobility of individual adatoms or adsorbed molecules remains largely unexplored. The lack of attention to the acoustic activation of surface diffusion can be explained by an intuitive expectation that, due to the large frequency mismatch between SAWs and vibrational states of the adsorbed species, the possibility of dynamic coupling of SAWs to the adsorbates can be excluded, whereas periodic perturbations of surface energy landscape associated with the acoustic strain should have a negligible effect on adatom mobility. This intuitive reasoning, however, comes into conflict with emerging experimental evidence of substantial enhancement of surface catalysis [131-136] and molecular desorption $[16-18,137,138]$ by acoustic waves, as well as recent observation of SAW-induced directional motion of small atomic Au clusters on a Si substrate[139]. These intriguing results call for a detailed analysis of the acoustic activation of atomic-level surface processes.

The results of a theoretical and computational investigation reported in this paper demonstrate that the enhancement of surface diffusion under the action of acoustic waves can reach tens and hundreds of percents. The mechanisms responsible for the enhancement of the surface diffusion of adsorbed atoms and the degree to which the diffusion can be affected/controlled by SAW are revealed in a systematic study that combines theoretical analysis with kinetic Monte Carlo (kMC) and molecular dynamics (MD) simulations. The prospects for the acoustic activation of surface diffusion illuminated in this study suggest an attractive alternative to the thermal activation in thin film growth on heat-sensitive substrates. 


\subsection{Mechanisms of acoustic activation of surface diffusion}

The modification of surface diffusion by SAW can be realized through the following three mechanisms: (1) modification of the diffusion barriers through transient variation of surface strain due to the SAW propagation, (2) variation of surface temperature due to the adiabatic heating and cooling associated with the fast surface deformation, and (3) dynamic coupling of the SAW to surface vibrational states of the adsorbed species. When considering the temperature dependence of the surface diffusion coefficient in the presence of a plane SAW propagating in $x$-direction, these three effects can be represented as follows:

$$
D(x, t)=D_{0} \exp \left(-\frac{E_{d}(x, t)}{k_{B} T(x, t)+\Delta E_{S A W}}\right) .
$$

Here, the SAW manifests itself via spatial and time variations of the surface temperature $T(x, t)$ and the diffusion barrier $E_{d}(x, t)$, as well as via the energy of the non-thermal vibrational excitation of the adsorbates $\Delta E_{S A W}$ due to the dynamic coupling with the SAW.

The dynamic coupling between the adsorbed species and SAW requires proximity to the "resonance condition," [16] i.e., the frequency of the SAW should be comparable to the ones of the surface vibrational states. While this requirement makes the dynamic coupling an unlikely mechanism for sub-GHz SAWs typically generated with conventional interdigital transducers, recent advances in the development of photo-acoustic methods for generation of SAWs with frequencies approaching $100 \mathrm{GHz}[124,125,140]$ suggest that the acoustic activation of this mechanism may be feasible, particularly for physisorbed molecules weakly bound to the surface. Therefore, the contributions from all three mechanisms are analyzed below and related to the parameters of SAWs and characteristics of material/adsorbate systems.

\subsubsection{Modification of diffusion barriers by surface strain}

The effect of the modification of diffusion barriers, $E_{d}(x, t)$, through transient variation of surface strain is related to the strong non-linearity of the dependence of the diffusion coefficient on the activation barrier. Even if the surface strain in SAW induces symmetric variation of the activation barrier around a zero-strain value, $E_{d}^{0}$, the exponential dependence on $E_{d}$ may still result in a strong enhancement of the diffusion coefficient. The effect of the surface 
strain on the diffusion barrier has been investigated in a number of quasi-static atomistic calculations [141-146]. The calculations performed with density-functional theory (DFT) [142,144-146] and classical MD [141,143] for adatom diffusion on $\mathrm{Si}$ and $\mathrm{Ge}$ (001) [141,145,146], Ag (111) [142], GaAs (100) [144] and Lennard-Jonesium (001) [143] surfaces predict that surface strain on the order of $10^{-3}-10^{-2}$ can result in the variation of $E_{d}$ by $5-10 \%$. Although for surfaces with complex anisotropic diffusion paths the effect of strain on surface energy landscape is complex and may depend on the strain type (biaxial or uniaxial) and direction [144-146], for a variety of material systems and surface strains up to several percents [142-146], the variation of the diffusion barriers can be relatively well approximated by a linear dependence, i.e.,

$$
E_{d}(e) \approx E_{d}^{0}+\gamma e,
$$

where $e$ is the magnitude of the surface strain, $\gamma$ is the coefficient of proportionality between the diffusion barrier and surface strain, and $E_{d}^{0}$ is the unperturbed diffusion barrier at $e=0$.

To complement the existing data on the strain dependence of the diffusion barrier and to ensure reliable interpretation of the results of MD simulations reported below, we performed a series of calculations of energy barriers for adatom diffusion on (001) surfaces of strained FCC substrates. The interatomic interactions in the substrate are described by the Lennard Jones (LJ) potential with energy and length parameters $\varepsilon$ and $\sigma$, a cutoff function suggested in Ref. [147], and a cutoff distance of $3 \sigma$. The adatom-substrate interaction is described by the same potential, except for the length parameter, $\sigma_{a-s}$, that is varied between $0.8 \sigma$ and $1.5 \sigma$ to study the effect of the size of the adsorbate on the strain dependence of the diffusion barrier. Regardless of $\sigma_{a-s}$, the adatom is assumed to have the same mass $m$ as the substrate atoms. The calculations are performed for a substrate composed of $12 \times 12 \times 12$ FCC unit cells, with periodic boundary conditions applied in $x$ and $y$ directions and free boundary conditions used in $z$ direction. The uniaxial lateral strain $e_{x x}$ ranging from -0.04 to 0.04 (negative values correspond to compression) is imposed by changing the size of the substrate in $x$ direction. The diffusion barrier is defined as the difference of the total energies of systems with the adatom located at the binding site $\left(E_{B}\right)$ and the saddle point $\left(E_{S}\right)$, i.e., $E_{d}=E_{S}-E_{B}$. The values of $E_{S}$ and $E_{B}$ are evaluated by fixing the lateral position $(x, y)$ of the adatom at the binding site or the saddle point of the (001) surface and 
allowing the system to relax (to achieve the energy minimum) while allowing the adatom to only move in $z$ direction. The values of $E_{d}$ are found to follow the linear scaling with strain given by Eq. (2) and the results of the calculations are summarized in Table 3.

\begin{tabular}{|c|c|c|c|c|c|c|c|c|c|c|}
\hline$\sigma_{a-s}$ & $0.8 \sigma$ & $0.9 \sigma$ & $1.0 \sigma$ & $\begin{array}{c}1.0 \sigma \\
(\mathrm{MD})\end{array}$ & $1.1 \sigma$ & $1.2 \sigma$ & $1.3 \sigma$ & $1.4 \sigma$ & $1.5 \sigma$ & $\begin{array}{c}1.5 \sigma \\
(\mathrm{MD})\end{array}$ \\
\hline$E_{d}^{0} / \varepsilon$ & 1.93 & 1.69 & 1.48 & 1.48 & 1.28 & 1.07 & 0.86 & 0.66 & 0.47 & 0.49 \\
\hline$\gamma / E_{d}^{0}$ & 1.37 & 1.07 & 1.09 & 1.03 & 1.31 & 1.54 & 1.98 & 2.15 & 2.64 & 2.57 \\
\hline
\end{tabular}

Table 3: The values of the diffusion barrier $E_{d}^{0}$ and scaling factor $\gamma$ in Eq. (2) obtained in quasistatic calculations and MD simulations (shaded cells) performed for different values of $\sigma_{a-s}$ and uniaxial lateral strain $e_{x x}$ ranging from -0.04 to 0.04 .

The diffusion barriers evaluated by an alternative method, based on the results of direct MD simulations of adatom diffusion, are also listed in Table 3 for $\sigma_{a-s}=\sigma$ and $\sigma_{a-s}=1.5 \sigma$. In this case, several series of simulations are performed at different temperatures. At each temperature, $N=120$ independent adatom trajectories with a length of $8400 \tau_{0}$ are obtained, where $\tau_{0}=\sqrt{m \sigma^{2} / \varepsilon}$ (for reference, the period of adatom vibrations is $\sim 1.2 \tau_{0}$ for $\sigma_{a-s}=\sigma$ and $\sim 2.8 \tau_{0}$ for $\left.\sigma_{a-s}=1.5 \sigma\right)$. The diffusion coefficients are determined from time dependences of the mean square displacements of the adatoms, $<\Delta \vec{r}(t)^{2}>\equiv \frac{1}{N \times N_{t}} \sum_{i=1}^{N} \sum_{j=1}^{N_{t}}\left(\vec{r}_{i}\left(t+t_{j}\right)-\vec{r}_{i}\left(t_{j}\right)\right)^{2}=4 D t$, where $\vec{r}_{i}(t)$ are "unfolded" true adatom positions not affected by the periodic boundary conditions. The diffusion barrier is then obtained from the Arrhenius plot for the temperature dependence of the diffusion coefficient. A good agreement between the values of $E_{d}^{0}$ and $\gamma$ obtained in quasi-static and dynamic calculations supports the notion of the involvement of the motions of substrate atoms in the adatom jumps, accounted for by the relaxation of the substrate in the evaluation of the saddle point energy in the quasi-static calculations. Contrary to the assessment of Ref. [143], a procedure where no relaxation of the substrate is done in the calculation of the saddle point energy leads to a substantial overestimation (up to $60 \%$ for $\sigma_{a-s}=1.5 \sigma$ ) of the diffusion barriers as compared to the MD simulations. 


\subsubsection{Temperature variation due to the adiabatic heating and cooling}

Turning to the second mechanism of the acoustic modification of the diffusion coefficient given by Eq. (1), the variation of surface temperature $T(x, t)=T_{0}+\Delta T(x, t)$ induced by the SAW can be evaluated by considering the nearly-adiabatic transient acoustic deformation of the surface region. For the reversible adiabatic heating and cooling, $\Delta T / T_{0}=-K_{b} \alpha e_{i i}^{S A W} / c_{V}[148]$. where $K_{b}$ is the bulk elastic modulus, $\alpha$ is the thermal expansion coefficient, $c_{V}$ is the specific heat capacity at constant volume, and $e_{i i}^{S A W}$ is the dilatation caused by the acoustic deformation. Considering a SAW running along [100] direction on a (001) surface of an FCC LJ crystal, the surface strain in the direction of the SAW propagation can be described as $e_{x x}(x, t)=e_{0} \sin (k x-\Omega t)$, where $e_{0}$ is the amplitude, $\Omega=2 \pi \mathrm{c} / \lambda$ is the angular frequency, $k=2 \pi / \lambda$ is the wave vector, $c$ is the propagation velocity, and $\lambda$ is the wavelength of the SAW. The dilatation in the vicinity of the free surface is defined by two strain components with

opposite signs, $e_{i i}^{S A W}=e_{x x}+e_{z z}=\left(\frac{C_{11}-C_{12}}{C_{11}}\right) e_{x x}$, and is smaller than the one for the bulk wave, $e_{i i}=e_{x x}$. Using the thermoelastic parameters of the $\mathrm{LJ}$ material, the dilatation and temperature variations can be expressed as $e_{i i}^{S A W} \approx 0.43 e_{x x}$ and $\Delta T / T_{0} \approx-1.56 e_{x x}$.

The adiabatic temperature variation can also be directly studied in MD simulations performed for the LJ system discussed above, where the running SAW is generated and maintained as illustrated in Fig. 8.1. The computational system consists of 31 atomic planes in $z$ direction, 24 atomic planes (12 FCC unit cells) in $y$ direction, and has a size equal to the wavelength $\lambda$ of the SAW in $x$ direction. The periodic boundary conditions are applied in $x$ and $y$ directions that are parallel to (001) free surface of the system. Prior to the introduction of the SAW, the substrate is equilibrated at a desired temperature. The SAW is then initialized by assigning displacements and additional velocities to all atoms in the substrate according to the analytical expressions for the Rayleigh wave in an FCC crystal [149]. The SAW is then maintained by applying the dynamic boundary conditions to the bottom 5 atomic planes and 4 partial vertical planes, where the atomic displacements follow the analytical expressions for the Rayleigh wave [149]. The dynamic boundary condition suppresses the generation of higher harmonics that distort the shape of the SAW, particularly in systems with small $\lambda$. To avoid 
heating of the substrate due to the dissipation of the SAW, the temperature of 14 atomic layers adjacent to the bottom part of the dynamic boundary is maintained with the Berendsen thermostat method [150], with the atoms subject to the dynamic boundary condition excluded from the thermostat region. The atoms in the 12 topmost atomic layers of the substrate undergo unconstrained free dynamics. The instantaneous local temperature is calculated from kinetic energies of atoms in rows along $y$ direction and the neighboring rows. The collective velocity due to the acoustic wave propagation is subtracted from atomic velocities used in the temperature calculation.

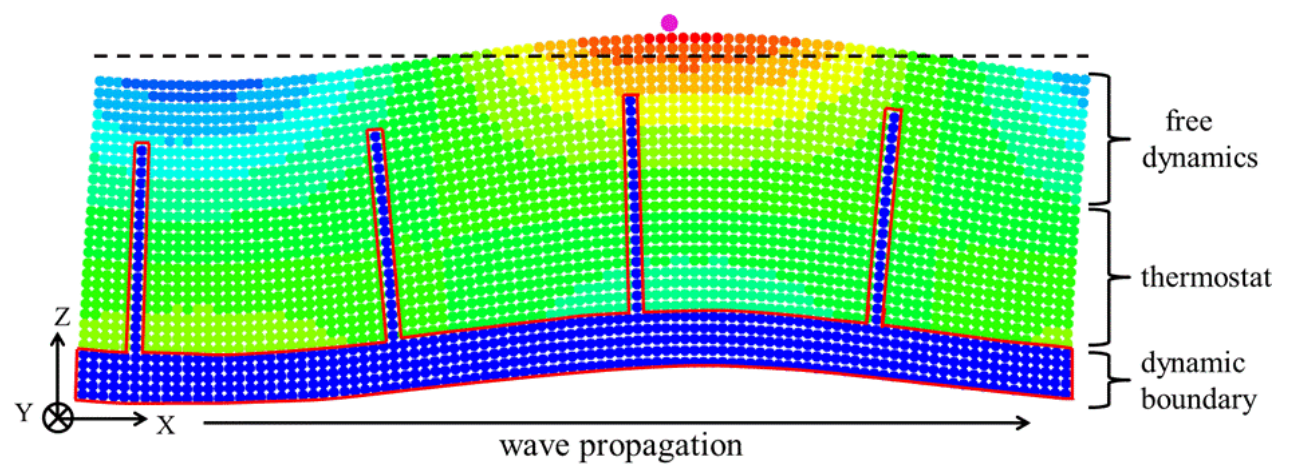

Figure 8.1: The computational system used in MD simulations of adatom diffusion on a substrate in the presence of a SAW propagating along the $x$ direction. The blue atoms in the region outlined by the red solid lines are displaced according to the analytical expressions for the desired Rayleigh wave. The purple atom is the adatom diffusing on the surface. The other atoms are colored by the instantaneous value of local strain. The atoms in the topmost 12 monolayers undergo unconstrained free dynamics and the atoms of the following 14 monolayers are coupled with a thermostat to keep a constant temperature of the system. The black dashed line marks the position of the free surface in the absence of SAW and the black arrow shows the wave propagation direction.

The results of the calculation of the instantaneous strain and temperature distributions in a MD simulation performed for a running SAW with the lateral surface strain amplitude $e_{0}=0.048$ and the wavelength $\lambda \approx 78 \sigma$ (50 FCC unit cells) are shown in Fig. 8.2. To reduce thermal noise in the strain and temperature distributions, the results are averaged over 138 periods of the wave. The profile of the surface strain shown in Fig. 8.2a follows closely the expected distribution of $e_{x x}(x, t)=e_{0} \sin (k x-\Omega t)$ at a fixed time $t$, whereas the profile of the 
surface temperature exhibits a small phase shift from the expected antiphase variation with strain. The phase shift and the asymmetry of the temperature distribution in Fig. 8.2c are related to deviations from perfectly adiabatic thermoelastic heating and cooling conditions in the case of high-frequency SAWs. The phase shift decreases with increasing $\lambda$ and, even for the small values of $\lambda$ used in this work, is found to have little effect on surface diffusion in kMC simulations. Therefore, the antiphase temperature and strain variations, i.e., $\Delta T / T_{0} \approx-\theta e_{x x}(x, t)$ are assumed in further discussion. The value of $\theta \approx 1.49$, obtained from about $7.2 \%$ magnitude of $\Delta T / T_{0}$ variation in Fig. 8.2, is in a fairly good agreement with the value of $\theta \approx 1.56$ estimated above based on thermoelastic properties of the substrate material.
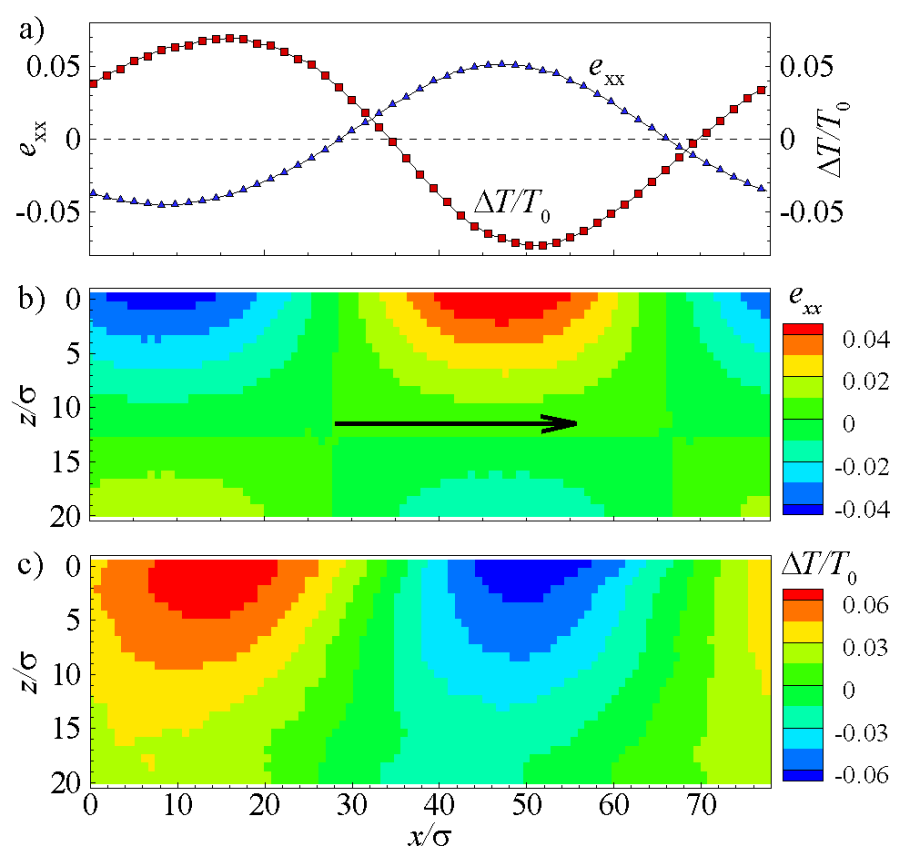

Figure 8.2: Instantaneous strain and temperature distributions in a MD simulation performed for a running SAW with $e_{0}=0.048$ and $\lambda \approx 78 \sigma$. The average temperature $T_{0}$ of the surface is maintained close to $T_{t h}=0.15 \varepsilon / k_{B}$. To reduce thermal noise in the strain and temperature distributions, the results are averaged over 138 periods of wave propagation. The strain and temperature in the top surface monolayer are shows in (a) by blue delta and red square symbols, respectively. The contour plots in (b) and (d) show the strain and temperature distributions in the sub-surface region of the substrate. The black arrow in (b) shows the direction of the wave propagation. 


\subsubsection{Diffusion enhancement via diffusion barrier and adiabatic temperature variations}

With the approximations of the linear scaling of the diffusion barrier, $E_{d}(x, t) \approx E_{d}^{0}+\gamma e_{x x}(x, t)$, and surface temperature, $\Delta T(x, t) / T_{0} \approx-\theta e_{x x}(x, t)=-\left[\gamma_{T} / E_{d}^{0}\right] e_{x x}(x, t)$ (where $\gamma_{T}=\theta E_{d}^{0}$ is introduced for convenience), the combined effect of the adiabatic temperature variation and the modification of the diffusion barriers can be evaluated by spatial and time integration of Eq. (1),

$$
D_{S A W}=\frac{1}{\lambda \tau} \int_{0}^{\lambda} d x^{\prime} \int_{0}^{\tau} d t^{\prime} D_{0} \exp \left(-\frac{E_{d}^{0}+\gamma e_{x x}\left(x^{\prime}, t^{\prime}\right)}{k_{B}\left[T_{0}+\Delta T\left(x^{\prime}, t^{\prime}\right)\right]}\right),
$$

where $\tau$ is the period of SAW. For small $\Delta T / T_{0}$, an approximation $1 /\left(1+\Delta T / T_{0}\right) \approx 1-\Delta T / T_{0}$ can be used and the above equation can be rewritten as

$$
D_{S A W}=\frac{1}{\lambda \tau} \int_{0}^{\lambda} d x^{\prime} \int_{0}^{\tau} d t^{\prime} D_{0} \exp \left(-\frac{\left[E_{d}^{0}+\gamma e_{x x}\left(x^{\prime}, t^{\prime}\right)\right]\left[1+\left[\gamma_{\mathrm{T}} / E_{d}^{0}\right] e_{x x}\left(x^{\prime}, t^{\prime}\right)\right]}{k_{B} T_{0}}\right) .
$$

By neglecting the second order term $\sim e_{x x}^{2}$ in the numerator of the exponent and considering an oscillating surface strain induced by SAW, $e_{x x}(x, t)=e_{0} \sin (k x-\Omega t)$, the diffusion coefficient can be expressed as

$$
D_{S A W}=\frac{D_{n}\left(T_{0}\right)}{(2 \pi)^{2}} \int_{0}^{2 \pi} d x^{\prime} \int_{0}^{2 \pi} d t^{\prime} \exp \left(-\frac{\gamma_{e f f} e_{0} \sin \left(x^{\prime}-t^{\prime}\right)}{k_{B} T_{0}}\right)=D_{n}\left(T_{0}\right) f\left(\gamma_{e f f} e_{0} / k_{B} T_{0}\right)
$$

where $D_{n}\left(T_{0}\right)=D_{0} \exp \left(-E_{d}^{0} / k_{B} T_{0}\right)$ is the diffusion coefficient in the absence of SAW and $\gamma_{e f f}=\gamma+\gamma_{T}$ is the effective scaling factor that reflects the additive contributions of the diffusion barrier and temperature variations to the diffusion enhancement. For positive values of $\gamma$, such as the ones listed in Table 3, the effect of the temperature variation acts synergistically with the diffusion barrier modification given by Eq. (2), i.e., the compressive strain $\left(e_{x x}<0\right)$ reduces the diffusion barrier $\left(E_{d}-E_{d}^{0}<0\right)$ while the simultaneous temperature increase $(\Delta T>0)$ further facilitates diffusion. Interestingly, the diffusion enhancement due to the transient variation of the diffusion barrier and temperature does not depend on the wavelength of the SAW, but is only a function of $\gamma_{e f f} e_{0} / k_{B} T_{0}$. 
The diffusion enhancement factor $D_{S A W} / D_{n}$ predicted by Eq. (5) is illustrated in Fig. 8.3 for a range of parameters that may be relevant to practical applications. The value of $\gamma_{\text {eff }}$ is intrinsic to a specific system and defines the limits of the diffusion enhancement that can be achieved at practically relevant values of $T_{0}$ and $e_{0}$. Since the temperature $T_{0}$ that ensures a $D_{n}$ that is sufficiently high to meet the requirements of a given application is determined by the magnitude of $E_{d}^{0}$, it is actually the value of $\gamma_{\text {eff }} / E_{d}^{0}$ that controls the diffusion enhancement for a given system. Assuming that an adsorbate makes one successful jump per $10^{4}$ "attempts" (jump rate $r^{*} \sim 0.1-1 \mathrm{~ns}^{-1}$ ) as an example, a complementary scale is shown on the right side of Fig. 8.3 to illustrate the diffusion barriers $E_{d}^{0^{*}}=4 k_{B} T_{0} \ln 10$ that ensure $r^{*}$ at $T_{0}$. The corresponding natural limit of $\gamma_{e f f} e_{0}<E_{d}^{0^{*}}$ is also outlined by a dashed line in Fig. 8.3. The upper limit for $e_{0}$ is determined by the onset of surface damage $[127,128]$ and is on the order of $10^{-2}$.

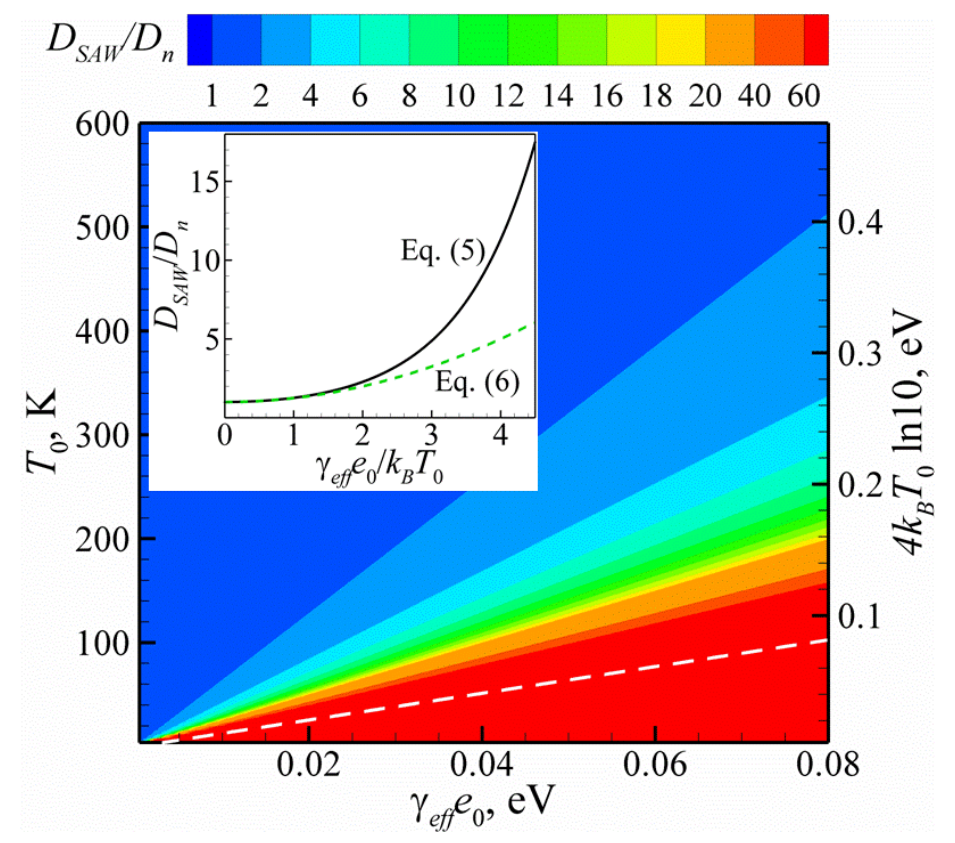

Figure 8.3: The diffusion enhancement factor $D_{S A W} / D_{n}$ as a function of $\gamma_{e f f} e_{0}$ and $T_{0}$ obtained by numerical integration of Eq. (5). The inset shows the diffusion enhancement as a function of $\frac{\gamma_{e f f} e_{0}}{k_{B} T_{0}}$ predicted by Eqs. (5) and (6). The scale on the right side of the contour plot shows the values of the diffusion barrier $E_{d}^{0^{*}}=4 k_{B} T_{0} \ln 10$ that ensure an adatom jump rate of about 
$r^{*} \sim 0.1-1 \mathrm{~ns}^{-1}$ at the corresponding substrate temperature $T_{0}$. The white dashed line in the contour plot corresponds to $\gamma_{e f f} e_{0}=E_{d}^{0^{*}}$.

To evaluate the approximate functional dependence of the diffusion enhancement factor $D_{S A W} / D_{n}$ on $\gamma_{e f f} e_{0} / k_{B} T_{0}$, the exponent in Eq. (5) can be expanded with respect to the small value of $\gamma_{e f f} e_{x x} / k_{B} T_{0}$, i.e., $\exp \left(-\gamma_{e f f} e_{x x} / k_{B} T_{0}\right) \approx 1-\gamma_{e f f} e_{x x} / k_{B} T_{0}+\left(\gamma_{e f f} e_{x x} / k_{B} T_{0}\right)^{2}$. After the spatial and time averaging in Eq. (5), this approximation yields the following scaling of the diffusion enhancement:

$$
D_{S A W} / D_{n} \approx 1+\frac{1}{4} \frac{\gamma_{e f f}^{2}}{\left(k_{B} T_{0}\right)^{2}} e_{0}^{2} .
$$

Thus, the diffusion enhancement can be expected for any sign of $\gamma_{\text {eff }}$ and exhibits quadratic scaling with $\gamma_{\text {eff }}$ and $e_{0}$. The relative change of the diffusion coefficient also scales as $T_{0}^{-2}$ and is larger for lower $T_{0}$, although the absolute change of the diffusion coefficient is larger at higher $T_{0}$ due to the Arrhenius temperature dependence of $D_{n}\left(T_{0}\right)$. As can be seen from the inset in Fig. 8.3, the discrepancy between the diffusion enhancement predicted by Eqs. (5) and (6) is increasing at low temperatures (large $\gamma_{e f f} e_{0} / k_{B} T_{0}$ ) due to the decreasing accuracy of the truncated Taylor expansion used in the derivation of Eq. (6).

The accuracy of the approximations used in the derivation of Eqs. (5) and (6) can be evaluated by performing one-dimensional (1D) kMC simulations of surface diffusion in the presence of a SAW. In kMC simulations, the microscopic kinetics of surface diffusion is reproduced by moving surface adatoms between the adjacent equilibrium sites with probability that is the product of the time step $\Delta t$ and the adatom jump rate, i.e., $p=R_{0} \exp \left[-E_{d}(x, t) / k_{B} T(x, t)\right] \times \Delta t$, where $R_{0}$ is the so-called attempt frequency related to the vibrational frequency of a surface adatom and chosen based on the value of $D_{0}$ predicted in MD simulations. The time step is chosen to ensure that $p<1$ and $\Delta t$ is at least one order of magnitude smaller than the period of SAW. The $\mathrm{kMC}$ simulations are performed under assumption of linear strain dependences of the diffusion barriers, $E_{d}(x, t)=E_{d}^{0}+\gamma e_{x x}(x, t)$, and surface temperature, $T(x, t)=T_{0}-T_{0} \theta e_{x x}(x, t)$, with surface strain associated with a running SAW 
introduced as $e_{x x}(x, t)=e_{0} \sin (k x-\Omega t)$. The local asymmetry of the energy barriers related to the gradient of the acoustic strain is neglected and the adatom has equal probability to jump to the left and to the right at any given time. The parameters of the simulations are chosen to match those determined in MD simulations performed for $\sigma_{a-s}=\sigma$ and $\sigma_{a-s}=1.5 \sigma$, with corresponding values of $E_{d}^{0}$ and $\gamma$ taken from the shaded cells of Table 3 and $\theta=1.49$ used to describe the acoustically-induced temperature variations. The distance between the equilibrium sites in 1D kMC simulations is chosen to be one half of the FCC lattice constant to allow for quantitative comparison with the results of MD simulations of adatom diffusion on a (001) surface of an FCC crystal. The total of 50,000 independent $1000 \Delta t$-long trajectories are generated in $\mathrm{kMC}$ simulations and, similarly to the MD simulations discussed above, the diffusion coefficient is calculated from the time dependence of the mean square displacement of the adatoms, $<\Delta x(t)^{2}>=2 D t$.

The diffusion enhancement found in kMC simulations and shown in Fig. 8.4 is consistently below the results of the numerical integration of Eq. (5). This is not surprising since both approximations used in the derivation of Eq. (5), the expansion $1 /\left(1+\Delta T / T_{0}\right) \approx 1-\Delta T / T_{0}$ and the neglect of a second order term $\sim e_{x x}^{2}$ in the numerator of the exponent, contribute to overestimation of the diffusion enhancement. The deviations, however, are relatively small in the whole range of temperatures considered in the calculations. This suggests that the approximations, which allowed us to describe the combined effect of the adiabatic temperature variation and the modification of the diffusion barriers in a uniform manner, through the introduction of $\gamma_{\text {eff }}$, are reasonable. The discrepancy between the predictions of Eq. (5) and Eq. (6) in Fig. 8.4 is also relatively small and, similarly to the inset of Fig. 8.3, increases with decreasing $T_{0}$. 

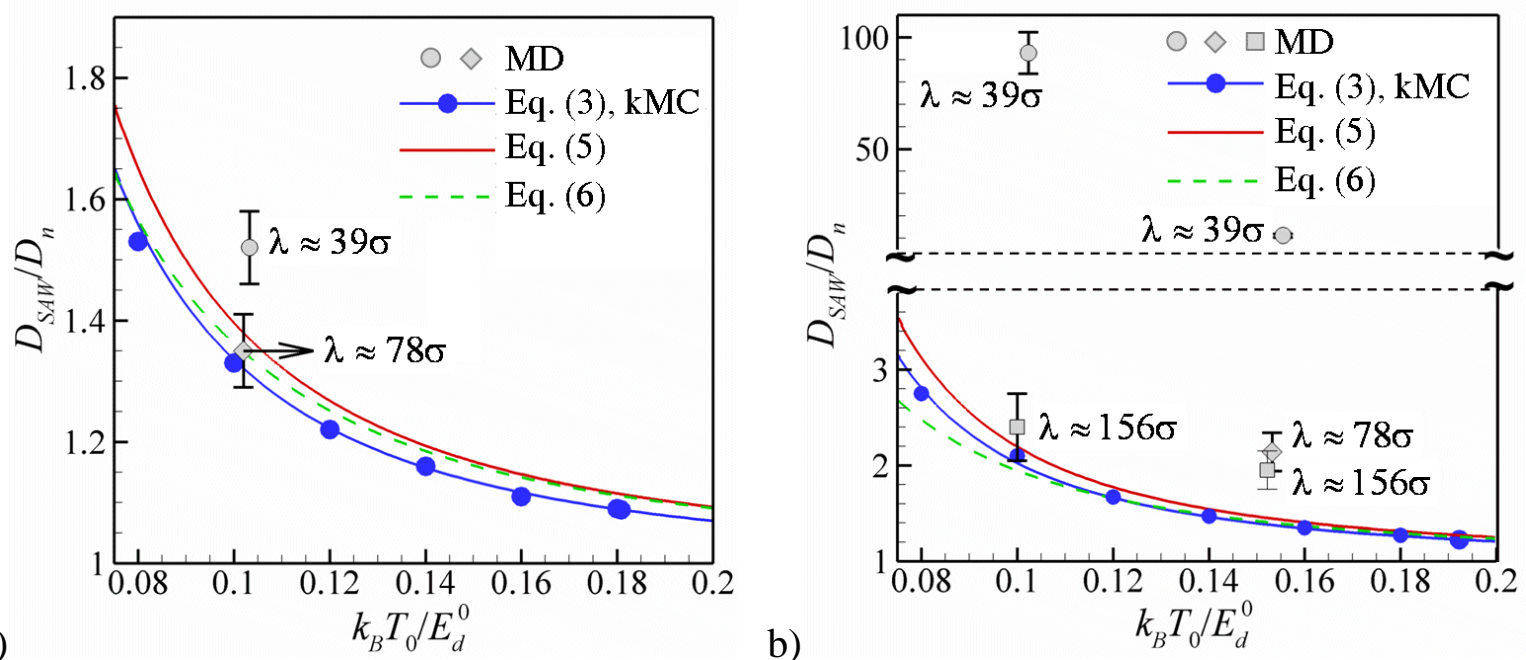

Figure 8.4: The diffusion enhancement $D_{S A W} / D_{n}$ by a SAW with $e_{0}=0.048$ as a function of $k_{B} T_{0} / E_{d}^{0}$ for adatoms with $\sigma_{a-s}=\sigma$ (a) and $\sigma_{a-s}=1.5 \sigma(\mathrm{b})$, respectively. The red solid line and the green dashed line show the predictions of Eqs. (5) and (6), respectively. The blue solid line and blue circles show the predictions of Eq. (3) and the results of kMC calculations, respectively. The large grey symbols show the results of MD simulations, with the error bars corresponding to the standard deviations of the mean calculated based on 8 independent series of the simulations.

\subsubsection{Dynamic coupling between adsorbates and SAW}

Finally, we turn to the third mechanism of the acoustic enhancement of surface mobility, the dynamic coupling between the adsorbed species and SAW leading to a non-thermal energy transfer to the adsorbate. The effect of dynamic coupling is represented in Eq. (1) as an extra energy $\Delta E_{S A W}$ added to the thermal energy of the adsorbates. Under conditions when the direct resonant coupling of the acoustic wave to the surface-adsorbate bond is prevented by the high frequency of individual vibrational surface modes, the energy transfer is likely to be a multi-step process involving anharmonic dissipation of the energy of the acoustic wave to the high frequency vibrations. A rigorous theoretical treatment of this process is difficult and, as the first step, we evaluate the contribution of the dynamic coupling to the diffusion enhancement by comparing the predictions of the KMC simulations discussed above with the results of MD simulations, which naturally account for the dynamic coupling, as well as the other two mechanisms that are reproduced in $\mathrm{kMC}$ simulations. 
The MD simulations with the same setup as used above in the analysis of the adiabatic temperature variation and illustrated in Fig. 8.1 are performed for adatoms with $\sigma_{a-s}=\sigma$ and $\sigma_{a-s}=1.5 \sigma$. For $\sigma_{a-s}=\sigma$, the simulations are done for two different wavelengths, $\lambda \approx 39 \sigma$ and $\lambda \approx 78 \sigma$, and the temperature of the thermostat region, $T_{\mathrm{th}}$, maintained at $0.1 E_{d}^{0} / k_{B}$. For $\sigma_{a-s}=1.5 \sigma$, two series of simulations with $\lambda \approx 39 \sigma$ and $\lambda \approx 78 \sigma$ are performed for $T_{t h}=0.1 E_{d}^{0} / k_{B}$ and three series of simulations with $\lambda \approx 39 \sigma, \lambda \approx 78 \sigma$ and $\lambda \approx 156 \sigma$ are performed for $T_{t h}=0.152 E_{d}^{0} / k_{B}$. Taking the value of the diffusion barrier $E_{d}^{0}=0.25 \mathrm{eV}$ as an example, the two temperatures used in the MD simulations correspond to 290 and $441 \mathrm{~K}$. Note that the energy release in the surface region of the substrate due to the dissipation of SAW leads to the fact that the steady-state surface temperature $T_{0}$ is slightly (up to $2 \%$ ) higher than the thermostat temperature $T_{t h}$ in the simulations performed with small $\lambda$. Therefore, the actual surface temperature $T_{0}$, rather than the thermostat temperature, is used in the presentation of the results of the MD simulations. The diffusion coefficients are calculated from the time dependences of the mean square displacements of the adatoms, based on the results of 512 independent trajectories obtained for each set of conditions (except for the simulation with $\sigma_{a-s}=1.5 \sigma, T_{t h}=0.1 E_{d}^{0} / k_{B}$, and $\lambda \approx 156 \sigma$, for which 1280 simulations were done). The duration of each simulation was $4200 \tau_{0}$ for $T_{t h}=0.1 E_{d}^{0} / k_{B}$ and $2100 \tau_{0}$ for $T_{t h}=0.152 E_{d}^{0} / k_{B}$.

The values of the diffusion enhancement obtained in MD simulations and shown in Fig. 8.4 demonstrate the convergence to the predictions of the $\mathrm{kMC}$ simulations at long wavelengths of SAWs, thus supporting the discussion of the wavelength-independent strain-induced mechanisms provided above. At the same time, the MD results demonstrate a substantial contribution of the dynamic effects to the diffusion enhancement at small wavelengths, especially for $\sigma_{a-s}=1.5 \sigma$. This large contribution of the dynamic effects is surprising given that even for the shortest wavelength $\lambda=39 \sigma$ the period of SAW is $\sim 7.6 \tau_{0}$, several times larger than the period of adatom vibrations, thus excluding the direct resonance coupling between the SAW and adatom vibrations. Spectral analysis of the atomic dynamics in the surface region of the substrate indicates that the generation of higher harmonics is largely suppressed by the dynamic boundary condition designed to maintain the desired shape of the SAW. Nevertheless, 
the presence of a weak signal from the $2^{\text {nd }}$ (and $3^{\text {rd }}$ in the case of $\lambda \approx 39 \sigma$ ) harmonics leaves the possibility of coupling of the higher harmonics to the adatom vibrations open. The particularly large diffusion enhancement observed for $\sigma_{a-s}=1.5 \sigma$ at $\lambda \approx 39 \sigma\left(D_{S A W} / D_{n}=90 \pm 9\right.$ at $T_{0}=0.102 E_{d}^{0} / k_{B}$ and $D_{S A W} / D_{n}=11.2 \pm 0.8$ at $\left.T_{0}=0.155 E_{d}^{0} / k_{B}\right)$, as well as the noticeable contribution of the dynamic effects to the diffusion enhancement even at $\lambda \approx 156 \sigma$ (Fig. 8.4b), can also be related to the relatively long lifetime of the excited vibrational state of the adatom. The lifetime of the excited state is evaluated by exponential fitting of the evolution of the amplitude of the kinetic energy oscillations of an adatom initially placed at a saddle point position on a quenched (zero temperature) substrate, as illustrated in Fig. 8.5. For $\sigma_{a-s}=1.5 \sigma$ the lifetime is found to be $\sim 30 \tau_{0}$, which corresponds to about 10 vibrational periods of the adatom and is comparable to the period of SAW with $\lambda \approx 156 \sigma$. The long "memory" of the vibrational excitation is reflecting the weak coupling between the adatom vibrations and substrate phonons and results in frequent multiple jumps (one per 2-3 jumps) observed in the simulations performed for $\sigma_{a-s}=1.5 \sigma$. On the contrary, for $\sigma_{a-s}=\sigma$ the lifetime of the excited vibrational state of the adatom is only $\sim \tau_{0}$, such that the contribution of the dynamic effects to the diffusion coupling disappears at $\lambda \geq 78 \sigma$ (Fig. 8.4a), and the multiple jumps are rare (e.g., 2 out of 100 jumps at $T_{0}=0.102 E_{d}^{0} / k_{B}$ ). 

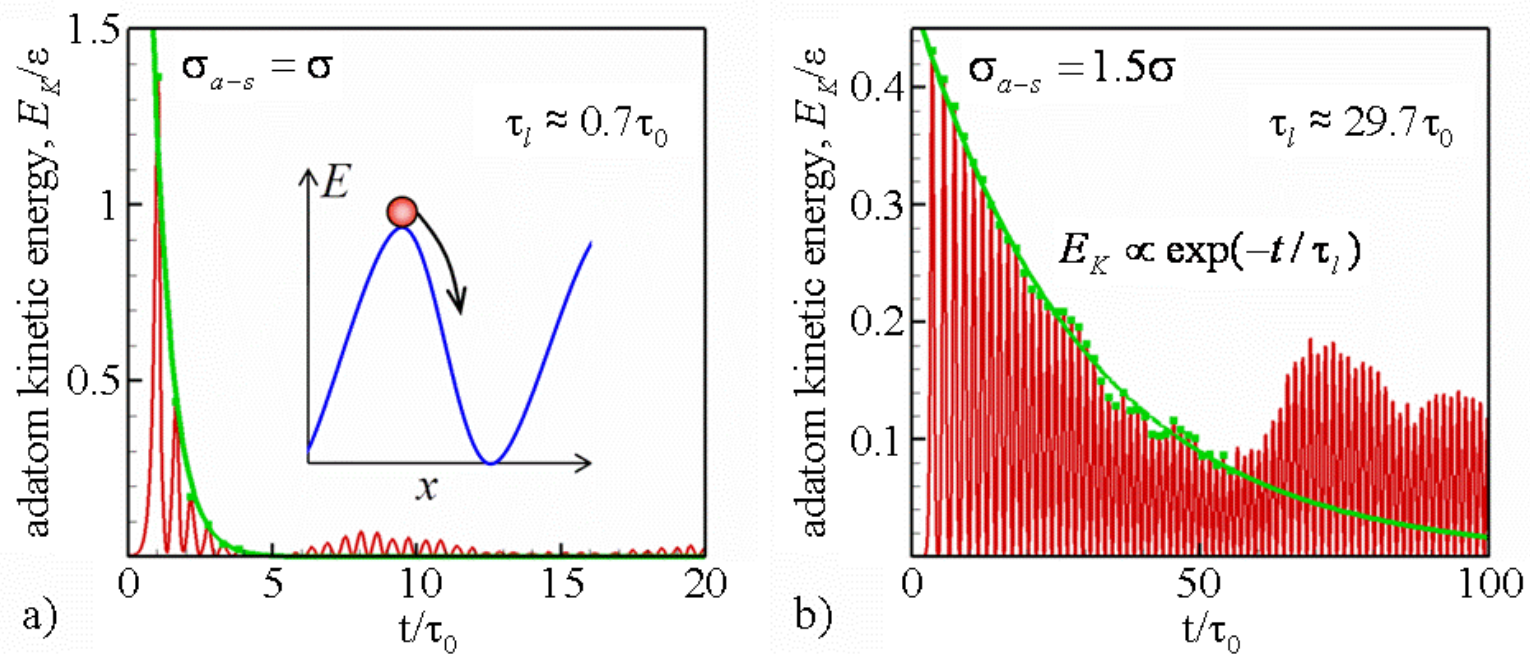

Figure 8.5: Evaluation of the lifetimes of the excited vibrational state of adatom with $\sigma_{a-s}=\sigma$ (a) and $\sigma_{a-s}=1.5 \sigma(\mathrm{b})$. The lifetime is obtained by exponential fitting of the evolution of the amplitude of the oscillations of the kinetic energy an adatom initially placed at a saddle point position on a quenched substrate, as shown schematically in the inset in (a). Green lines show the results of the exponential fitting, with data from the initial parts of the simulations $\left(4 \tau_{0}\right.$ for $\sigma_{a-s}=\sigma$ and $54 \tau_{0}$ for $\left.\sigma_{a-s}=1.5 \sigma\right)$ used in the fitting. The long-term beatings of the kinetic energy are related to the interaction of weak acoustic pulses generated by the excited adatom with periodic boundary conditions used in the calculations (the substrate represented by $12 \times 12 \times 12$ FCC unit cells is used in these calculations).

\subsection{Concluding remarks}

The results of the theoretical analysis and atomistic simulations reported above demonstrate the feasibility of the idea of acoustic activation of surface diffusion and identify the mechanisms responsible for the diffusion enhancement. Transient modification of the diffusion barriers and the reversible/adiabatic temperature variation due to the periodic oscillations of the acoustic strain are shown to be capable of manyfold enhancement of surface diffusion for systems that exhibit strong strain dependence of the diffusion barriers. Note that the system considered in this work, an adatom on a (001) surface of a LJ crystal, is far from being unique in demonstrating the diffusion enhancement, as the values of $\gamma / E_{d}^{0}$ reported for various systems 
can be substantially larger than those listed in Table 3. In particular, a broad range of values of $\gamma / E_{d}^{0}$ has been reported for biaxially strained substrates, e.g., 8.6 for $\mathrm{Ag} / \mathrm{Ag}(111)$ [142], -5 for $\mathrm{Ge} / \mathrm{Si}(001)$ [146], -15 for $\mathrm{Ge} / \mathrm{Ge}(001)$ [146], -2.7 for $\mathrm{In} / \mathrm{GaAs}(001)$ [144], and from -6.8 to 3.4 (for different diffusion paths) for $\mathrm{Si} / \mathrm{Si}(001)$ [145]. The value of $\gamma$ calculated in our work for the uniaxially strained substrate with $\sigma_{a-s}=\sigma$ is about twice smaller than the value reported for the same system under conditions of biaxial strain [143] suggesting that, in accord with similar observations for $\mathrm{Si}(001)$ surface [145], the components of surface strain make a near-additive contributions to the scaling of the diffusion barrier.

An approximate scaling law established in the theoretical analysis reveals the connections between the diffusion enhancement due to the transient variation of the acoustic strain and the parameters characterizing the acoustic wave and surface-adatom interaction. Importantly, the scaling law predicts independence of the strain-induced enhancement on the frequency of the SAW. An additional mechanism of acoustic activation of surface diffusion that does exhibit strong frequency dependence is the dynamic coupling of SAW to the vibrational states of the adsorbed species. More than an order of magnitude diffusion enhancement is observed in MD simulations when the frequency of the SAW is about 3 times lower than the vibrational frequency of the adsorbate. While the contribution of the dynamic coupling to the diffusion enhancement diminishes with decreasing frequency of SAW, the presence of this effect down to the frequency of an order of magnitude lower that the vibrational frequency of the adsorbate, combined with the large magnitude of the dynamic diffusion enhancement, suggest the potential practical importance of this mechanism in applications utilizing high-frequency SAWs in the range of tens of $\mathrm{GHz}[124,125,140]$.

Overall, the results of this study reveal the fundamental physical mechanisms of acoustic activation of surface diffusion and establish the dependence of the surface processes on the parameters of the acoustic waves. The acoustic activation of surface processes can serve as an attractive alternative to the conventional thermal activation in thin film growth on heat-sensitive substrates, and the mechanistic insights obtained in this study provide guidelines for the design of novel film growth techniques. 


\section{Mechanism of acoustically induced diffusional structuring of surface adatoms}

\subsection{Introduction}

The phenomenon of average forces acting on particles in oscillating fields has long been attracting interest [1-3] that is renewed now [154] in view of novel lab-on-a-chip applications, e.g., for structuring of particles suspended in liquids and non-contact manipulation of acoustically trapped individual micro-particles [129,155,156]. A discussion in [153] of the appearance of time-averaged forces acting on particles subjected to symmetric-in-time but spatially non-uniform oscillating force emphasizes that, in the absence of other forces, an oscillating particle tends to positions where its kinetic energy is minimal. Thus, the kinetic energy of the particle oscillations plays the role of an effective potential. Hereafter, we call this mechanism inertial, since inertia (non-zero particle's mass) is essential for appearance of the average force. A manifestation of this mechanism is gathering of charged particles in nodes of an oscillating electric field regardless of the sign of the charge [157,158]. In the presence of other potential forces, new equilibrium positions can appear for the total effective potential. This is exemplified by a well-known case of a rigid pendulum with the oscillating suspension point, for which counter-intuitive local equilibrium at the upper point may appear [153].

Another commonly discussed mechanism of average-force appearance is the radiation mechanism $[151,152,154]$. Unlike the inertial one, it can operate even for spatially uniform, on average, running fields. Scattering of the incident wave by a particle causes the mechanical momentum transfer and results in the appearance of time-averaged force. Under certain conditions, the radiation force may act in accord with the inertial one. For example, particles in liquids subjected to acoustic waves can experience both the inertial force (due to the difference in densities of the liquid and particles) and the radiation force (due to the difference in the compressibility, even if the densities are equal). This interplay determines the so-called Gorkov's acoustic radiation-force potential for particles in liquids [159] and its extensions accounting for viscosity $[154,160]$.

Recently, intriguing results of molecular dynamics (MD) simulations were reported for even smaller objects: nanostructuring of adatoms was induced by a standing SAW on a solid 
substrate [161]. Since for individual atoms radiation pressure due to acoustic wave re-radiation is irrelevant, the physical origin of the computational results [161] was discussed in Ref. [161-163] within the framework of the attractively clear inertial mechanism which, in principle, is applicable to individual ions and electrons (e.g., in plasmas) $[157,158]$. However, despite the apparent qualitative similarity between the results of the theoretical analysis $[162,163]$ and MD simulations [161], the questions on the dependence of the characteristics of nanostructuring on the parameters of SAW and, more generally, on applicability of the inertial mechanism to realistic material systems remain open.

In this section, we report an approximate analytical model and results of kinetic MonteCarlo (kMC) simulations which reveal a specific, essentially diffusional, mechanism of acoustically-induced time-averaged structuring of surface adatoms. This mechanism cannot be reduced to conventionally used paradigms of inertia- and radiation-induced average forces and, to the best of our knowledge, has not been discussed earlier. We found an explicit relationship between the acoustic strains and their average action, which revealed a rich variety of possible structuring regimes. Before turning to the discussion of the diffusional mechanism, we briefly recall some of the features of the inertial mechanism that make it inoperable for adatom diffusion and necessitate the search for an alternative mechanism.

\subsection{The role of damping}

Certainly, the mere presence of damping does not necessarily deem the inertial mechanism impractical. To evaluate its efficiency for a particle of mass $m$ oscillating in the presence of damping with effective coefficient $\alpha$, we consider a representative case of a sinusoidal standing wave force field with amplitude $F_{0}$, frequency $\Omega$, and wavenumber $k$, described by the following equation:

$m \ddot{x}+\alpha \dot{x}=F_{0} \cos (\Omega t) \sin (k x)$.

To obtain the time-averaged motion, the solution can be represented as a sum $x(t)=X(t)+\zeta(t)$ of the slowly varying term $X(t)$ and rapidly oscillating term $\zeta(t)$. Conventional procedures $[153,161]$ yield the following solution for $\zeta(t)$ in the first-order of $F_{0}$ :

$\zeta(t)=-\frac{F_{0} \sin (k X) m}{\alpha^{2}+m^{2} \Omega^{2}} \cos (\Omega t)+\alpha \frac{F_{0} \sin (k X)}{\alpha^{2}+m^{2} \Omega^{2}} \frac{\sin (\Omega t)}{\Omega}$. 
For small-amplitude oscillations $\zeta k<<1$, the slow part of the particle motion, $X(t)$, obeys the equation with the same lhs as Eq.(1) and time-averaged rhs:

$$
m \ddot{X}+\alpha \dot{X} \approx<k \zeta(t) F_{0} \cos (\Omega t) \cos [k X(t)]>\equiv-\frac{\partial W_{e f f}}{\partial X},
$$

where the effective potential $W_{\text {eff }}$ for the slow motion is:

$W_{\text {eff }}=\frac{1}{4} \frac{m F_{0}^{2}}{\alpha^{2}+m^{2} \Omega^{2}} \sin ^{2}(k X)$.

Notice that, since $<\cos (\Omega t) \sin (\Omega t)>=0$, the second term in Eq. (2) (that is in quadrature with the force) does not contribute to $W_{\text {eff }}$. This means that increasing $\alpha$ shifts $\zeta(t)$ out of phase with the force and eliminates the effective potential for $\alpha>>\Omega m$. In energy terms, this means that the role of kinetic energy of the primary oscillatory motion of the particle becomes negligible (inertia is small compared with viscous forces). For given $\Omega$ and $m$, Eq. (4) indicates that the damping coefficient should be sufficiently small, i.e. $\alpha / m<<\Omega$, for the inertial effects to become prominent (alternatively, for a fixed $\alpha$, the dominance of inertia means $\left.\Omega \gg \Omega^{*}=\alpha / m\right)$.

To determine whether the inertia dominates, one needs to estimate the effective damping coefficient $\alpha$ for diffusing adatoms. To this end, one may turn to the classical Langevin equation, $m \ddot{x}+\alpha \dot{x}=f(t)$, describing diffusional motion of a particle under the action of a random force $f(t)$ mimicking the thermal fluctuations. The effective damping coefficient $\alpha$ in Eq. (1) has the same physical meaning as in the Langevin equation, for which the value of $\alpha$ can be related to the diffusion coefficient $D$ at temperature $T$ through the Einstein-Smoluchowski relation [164]: $\alpha=k_{B} T / D$, where $k_{B}$ is the Boltzmann constant.

Even for an active diffusion with $D \sim 10^{-10} . .10^{-9} \mathrm{~m}^{2} / \mathrm{s}$, temperature of hundreds of $\mathrm{K}$, and adatom mass of $\sim 100 \mathrm{amu}$, the condition for significance of the inertial terms is $\Omega>>\Omega^{*}=\alpha / m \sim 10^{14} .10^{15} \mathrm{~s}^{-1}$. Such a frequency of the acoustic wave required for the dominance of the inertial effects is several orders of magnitude higher than the frequency of atomic vibrations (the Debye frequency is $\Omega_{D} \sim 10^{13} \mathrm{~s}^{-1}<\Omega *$ ). This suggests that for any SAW 
with a realistic frequency, the inertial mechanism of generation of time-averaged forces is completely suppressed for the diffusional motion of surface adatoms.

\subsection{Diffusional mechanism}

Since the commonly discussed inertial and radiation-force mechanisms are not relevant to the acoustically induced structuring of surface adatoms, we now focus on revealing an alternative mechanism that is consistent with diffusional nature of adatoms mobility. As a starting point, we note that the acoustic strain $e(x, t)$ can modify the instantaneous values of both the diffusion barriers $E_{d}$ and surface temperature $T$, thus affecting the adatom jump rate determined by the Arrhenius law

$$
r(x, t)=R_{0} \exp \left(-\frac{E_{d}[e(x, t)]}{k_{B} T[e(x, t)]}\right)
$$

where $R_{0}$ is the so-called attempt frequency related to the vibrational frequency of a surface adatom.

The effect of strain on the energy landscape for adatom diffusion is schematically illustrated in Fig. 9.1. The results of quasi-static ab initio [142,144-146] and classical [143] atomistic calculations suggest that, for a broad range of material systems, the variation of the binding energy $E_{B}$ and the saddle point energy $E_{S}$ of an adatom on a strained surface can be fairly well approximated by linear dependences up to surface strains of several percents, i.e., $E_{B}(e) \approx E_{B}^{0}+\beta_{B} e$ and $E_{S}(e) \approx E_{S}^{0}+\beta_{S} e$, where $E_{B}^{0}$ and $E_{S}^{0}$ are the binding and saddle point energies in the absence of strain. The strain dependence of the corresponding diffusion barrier, $E_{d}=E_{S}-E_{B}$, can be described as

$E_{d}(e) \approx E_{d}^{0}+\gamma e$

where $E_{d}^{0}$ is the diffusion barrier at a strain-free surface and $\gamma=\beta_{S}-\beta_{B}$. 


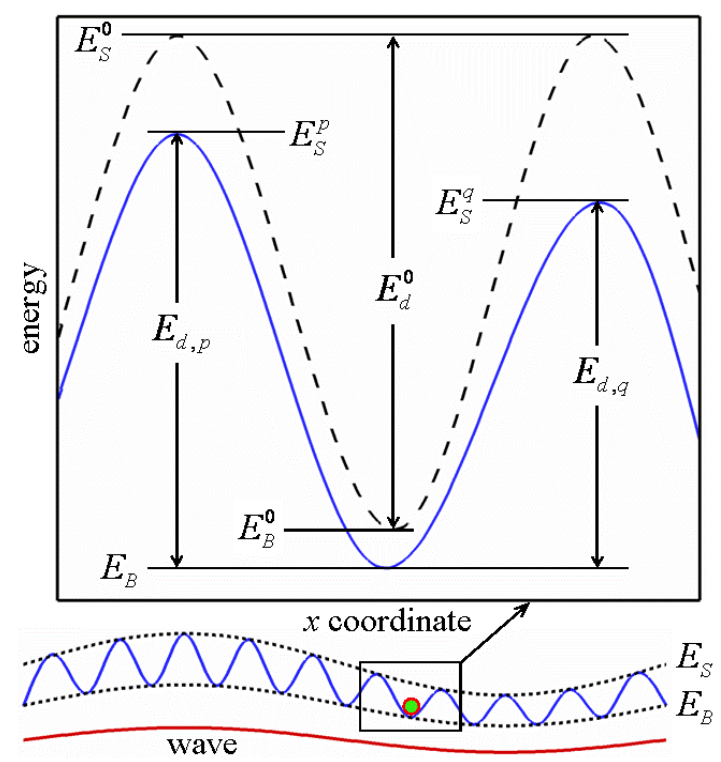

Figure 9.1: Schematic illustration of the effect of surface strain generated by a SAW on the energy landscape for adatom diffusion. The dashed and solid lines show the energy barriers for adatom diffusion without and with the SAW, respectively.

For inhomogeneous uniaxial strain $e=e(x, t)$, such as the one generated by SAWs, the modification of the surface energy landscape is not limited to the variation of the local diffusion barrier given by Eq. (6) but also includes the asymmetry of the diffusion barriers for adatom jumps along and counter the strain gradient, i.e., $E_{d, p}(x)$ and $E_{d, q}(x)$ for jumps in the left and right directions in Fig. 9.1:

$E_{d, q}(x)-E_{d, p}(x) \approx \delta \cdot b \cdot \partial e / \partial x$

Here, $b$ is the adatom jump distance in $x$ direction and $\delta$ is the diffusion barrier asymmetry parameter that can readily be shown to be equal to $\beta_{S}$. As demonstrated below, it is the interplay of the diffusion barrier inhomogeneity [Eq. (6)] and asymmetry [Eq. (7)] that largely defines the characteristics of the acoustically induced structuring of surface adatoms.

Besides the modification of the surface energy landscape, the adatom jump rate given by Eq. (5) can be affected by transient changes of surface temperature associated with the variation of the acoustic strain. The reversible adiabatic heating and cooling [165] occur even if the substrate acts as an efficient thermostat and trivial heating due to the wave dissipation is 
negligible. The nearly-adiabatic variations in $T$ are approximately linear in strain and can be expressed as

$T(e) \approx T_{0}-T_{0} \theta e(x, t)$

where $T_{0}$ the temperature of the substrate and the parameter $\theta$ is defined by the elastic moduli, heat capacity and coefficient of thermal expansion of the substrate material [165]. For small changes of the surface temperature $\left(\Delta T<<T_{0}\right)$, the effect of the adiabatic temperature variation on the adatom jump rate given by Eq. (5) can be approximated by an equivalent variation in the diffusion barrier, $E_{d}^{T}(e) \approx E_{d}^{0}+\gamma_{T} e$, where $\gamma_{T}=\theta E_{d}^{0}$ [166]. By introducing $\gamma_{\text {eff }}=\gamma+\gamma_{T}$, the combined effect of the adiabatic temperature variation and the modification of the diffusion barriers given by Eq. (6) can then be described in terms of an effective variation in the diffusion barrier,

$E_{d}^{e f f}(e) \approx E_{d}^{0}+\gamma_{e f f} e$

Now, with the understanding of the strain effect on key parameters controlling diffusion, the time-averaged structuring of adatoms in the presence of a standing SAW can be considered by solving $1 \mathrm{D}$ kinetic equation

$\partial n(x) / \partial t=v_{+}-v_{-}$,

where $n(x)$ is the adatom density at a site located at $x, v_{-}(x)=n(x)\left[r_{p}(x)+r_{q}(x)\right]$ and $v_{+}(x)=n(x+b) r_{p}(x+b)+n(x-b) r_{q}(x-b)$ are the rates of adatom jumps from/to neighboring sites with coordinates $x \pm b, r_{p}$ and $r_{q}$ are the jump rates to the left and to the right given by Eq. (5) with corresponding barriers $E_{d, p}$ and $E_{d, q}$ that are perturbed by acoustic strain $e(x, t)$. In what follows, by analogy with the rhs of Eq. (1), we consider an oscillating strain field that corresponds to a standing SAW, $e(x, t)=e_{0} \cos (\Omega t) \sin (k x)$.

We denote the uniform unperturbed density as $n_{0}$ and its dimensionless perturbation as $N=\left(n-n_{0}\right) / n_{0}$. Then, recalling that for acoustic waves $k b<<1$, combining Eq. (10) with Eqs. (5), (7), and (9), and expanding the exponent in Eq. (5) to the quadratic in $e_{0}$ and $k b$ perturbations in $E_{d}^{e f f}$, one obtains the following diffusion equation for $N$ : 


$$
\begin{aligned}
& \frac{\partial N}{\partial t}-r_{0} b^{2} \frac{\partial^{2} N}{\partial x^{2}} \approx b^{2} \frac{\partial^{2}}{\partial x^{2}}[R(x, t)+R(x, t) N]+ \\
& +b \frac{\partial}{\partial x}[Q(x, t)+Q(x, t) N]
\end{aligned},
$$

where $r_{0}$ is the unperturbed jump rate given by Eq. (5) at $e=0$, and the acoustically induced "force" in the right- hand side is defined by $R(x, t)=\left(r_{p}+r_{q}\right) / 2-r_{0}$ and $Q(x, t)=r_{p}-r_{q}$ that characterize the variations in the adatom jump rates due to the acoustically induced modification of the magnitudes of the effective diffusion barriers [Eq. (9)] and their left-right asymmetry [Eq. (7)], respectively. If the exponent in Eq. (5) is expanded to the second order in strain, $R(x, t)$ and $Q(x, t)$ can be approximated as

$$
\begin{aligned}
& R \approx R^{(1)}+R^{(2)}=-\frac{r_{0} \gamma_{e f f} e}{k_{B} T_{0}}+\frac{r_{0}}{2}\left(\frac{\gamma_{e f f} e}{k_{B} T_{0}}\right)^{2}, \\
& Q \approx Q^{(1)}+Q^{(2)}=\frac{r_{0} b \delta \partial e / \partial x}{k_{B} T_{0}}-\frac{r_{0} b \delta \gamma_{e f f} e \partial e / \partial x}{\left(k_{B} T_{0}\right)^{2}} .
\end{aligned}
$$

The "force" in the rhs of Eq. (11) comprises two terms that do not depend on $N$ and are directly determined by the strain-dependent perturbation of the Arrhenius jump rates described by the quantities $R$ and $Q$, which are approximated by Eqs. (12) and (13). The other two terms contain products $R N$ and $Q N$ and are due to the fact that the rates $v_{ \pm}$in the kinetic equation (10) are proportional to the local density of the adatoms and the probabilities of leaving or entering a given atomic site.

For an oscillating acoustic strain field, the linear-in-strain components $R^{(1)}, Q^{(1)}$ entering the rhs of Eq. (11) yield the linear-in-strain oscillating perturbation $N^{(1)}$ of the adatom density. Then, in the next approximation, quadratic-in-strain terms $R^{(1)} N^{(1)}$ and $Q^{(1)} N^{(1)}$ in Eq. (11) lead to the time-averaged (over a SAW period) perturbation of adatoms' concentration. This "cascade" mechanism, however, is extremely damped for any realistic SAW parameters and, for a given $k$, it can be activated only for $\Omega \rightarrow 0$, i.e., for static periodic strain $e(x)=e_{0} \sin (k x)$ rather than oscillating acoustic strain. While this mechanism may control nucleation of selfassembled islands on surfaces where static periodic strain field is created by dislocation arrays 
[167] or buried strained islands $[168,169]$, it does not contribute to the acoustic structuring and is not considered further in the present paper.

The quadratic-in-strain terms $\left(R^{(2)}\right.$ and $\left.Q^{(2)}\right)$ entering the rhs of Eq. (11) due to nonlinearity of the Arrhenius rate equation, on the other hand, directly yield non-zero perturbation in the adatoms' concentration averaged over the SAW period, $\bar{N}$. The leading term that provides an estimate for the time-scale and magnitude of structuring induced by the acoustic strain has the following form

$\bar{N}(x, t) \approx[1-\exp (-t / \tau)] \frac{\gamma_{e f f}\left(\gamma_{e f f}-\delta\right)}{8\left(k_{B} T_{0}\right)^{2}} e_{0}^{2} \cos (2 k x)$,

where the characteristic structuring time $\tau=1 /\left(4 b^{2} k^{2} r_{0}\right)$. The more detailed theoretical derivation can be found in Appendix C.

Several conclusions can be derived from the approximate scaling law given by Eq. (14) for the acoustic structuring.

First, similarly to the inertial mechanism described by Eq. (1), the spatial period of the diffusional structuring is twice smaller than the wavelength of SAW, $\lambda=2 \pi / k$. This similarity prevents the discrimination of the mechanisms by the period of structuring and is due to the trivial reason that both effects in the lowest approximations are quadratic in strain.

Second, the wavelength of SAW does not affect the ultimate structuring contrast $\bar{N}(x, t \rightarrow \infty)$ but defines the characteristic structuring time $\tau$. This is an important conclusion indicating that the effect of surface structuring is not limited to extreme frequencies of 100s of $\mathrm{GHz}$ [161] and can be achieved with conventional sources of SAWs. As illustrated in Fig. 9.2, the quadratic increase of the structuring time with wavelength, $\tau \sim \lambda^{2}$, characteristic of the diffusional redistribution of the adatoms, can be counteracted by a moderate temperature increase that affects the structuring time through the strong Arrhenius temperature dependence of $r_{0}$ given by Eq. (5) with $e=0$. The increase in $T_{0}$ reduces the ultimate structuring contrast [see Eq. (14)] and the choice of $T_{0}$ is defined by the balance between the sufficiently high level of structuring contrast and reasonably short structuring time. Since the temperature $T_{0}$ that yields the value of $r_{0}$ needed to ensure a reasonable time of structuring for given parameters of the 
SAW is determined by the magnitude of $E_{d}^{0}$, the material parameter that controls the structuring is $\gamma_{\text {eff }}\left(\gamma_{\text {eff }}-\delta\right) /\left(E_{d}^{0}\right)^{2}$.

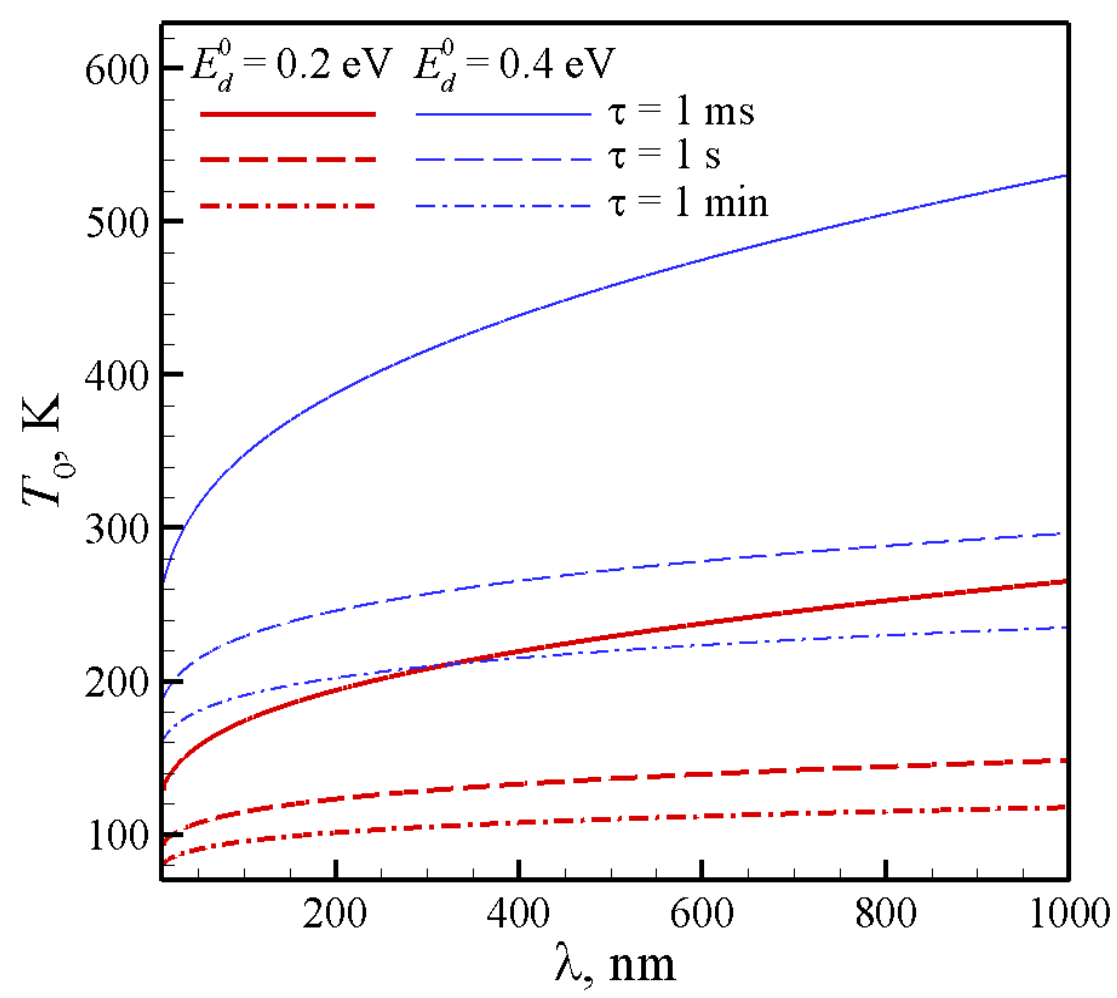

Figure 9.2: Isochrone curves of the structuring time $\tau$ as functions of the substrate temperature $T_{0}$ and SAW wavelength $\lambda=2 \pi / k$ for representative values of $b=0.2 \mathrm{~nm}, R_{0}=1 \mathrm{ps}^{-1}$, and $E_{d}^{0}$ equal to 0.2 , and $0.4 \mathrm{eV}$, as predicted by the analytical model, Eq. (14). The solid, dashed and dash-dotted curves are for $\tau$ equal to $1 \mathrm{~ms}, 1 \mathrm{~s}$, and $1 \mathrm{~min}$, respectively.

Finally, the factor $\gamma_{\text {eff }}\left(\gamma_{\text {eff }}-\delta\right)$ in Eq. (14) suggests that an interplay between straininduced variations of surface temperature [Eq. (8)] and magnitude of diffusion barriers [Eq. (6)], as well as local asymmetry of the barriers associated with the gradient of the acoustic strain [Eq. (7)] results in the existence of several distinct regimes of surface structuring, namely:

(i) gathering of adatoms in the regions of SAW-strain nodes when $\left(\gamma_{\text {eff }}>0\right.$ and $\left.\gamma_{\text {eff }}>\delta\right)$ or $\left(\gamma_{\text {eff }}<0\right.$ and $\gamma_{e f f}<\delta$ ), e.g., Fig. 9.3a;

(ii) gathering of adatoms in the regions of SAW-strain antinodes when $\left(\gamma_{\text {eff }}<0\right.$ and $\left.\gamma_{\text {eff }}>\delta\right)$ or $\left(\gamma_{\text {eff }}>0\right.$ and $\left.\gamma_{\text {eff }}<\delta\right)$, e.g., Fig. 9.3b; 
(iii) absence of structuring when the effective barrier-height variation is either absent $\left(\gamma_{\text {eff }}=0\right)$ or is compensated by the barrier asymmetry $\left(\gamma_{e f f}=\delta \neq 0\right)$.

The accuracy of the approximations used in the derivation of Eq. (14) can readily be evaluated in one-dimensional kMC simulations of surface diffusion [166] in the presence of a standing SAW. The simulations directly implement the discrete kinetic Eq. (10) in which the Arrhenius dependence of adatom jump rates [Eq. (5)] is retained without quadratic-in-strain expansion and the strain-induced variations of the diffusion barrier and temperature are described by Eqs. (6-8). The results of $\mathrm{kMC}$ simulations performed for two distinct regimes of surface structuring are shown in Fig. 9.3 along with the theoretical predictions. Both the time evolution of the density profiles and the final structuring contrasts predicted in the simulations are well described by the theoretical curves. Minor discrepancy between the approximate analytical and $\mathrm{kMC}$ results is mainly related to the quadratic-in-strain expansion of the Arrhenius equation that becomes less accurate at lower temperatures.
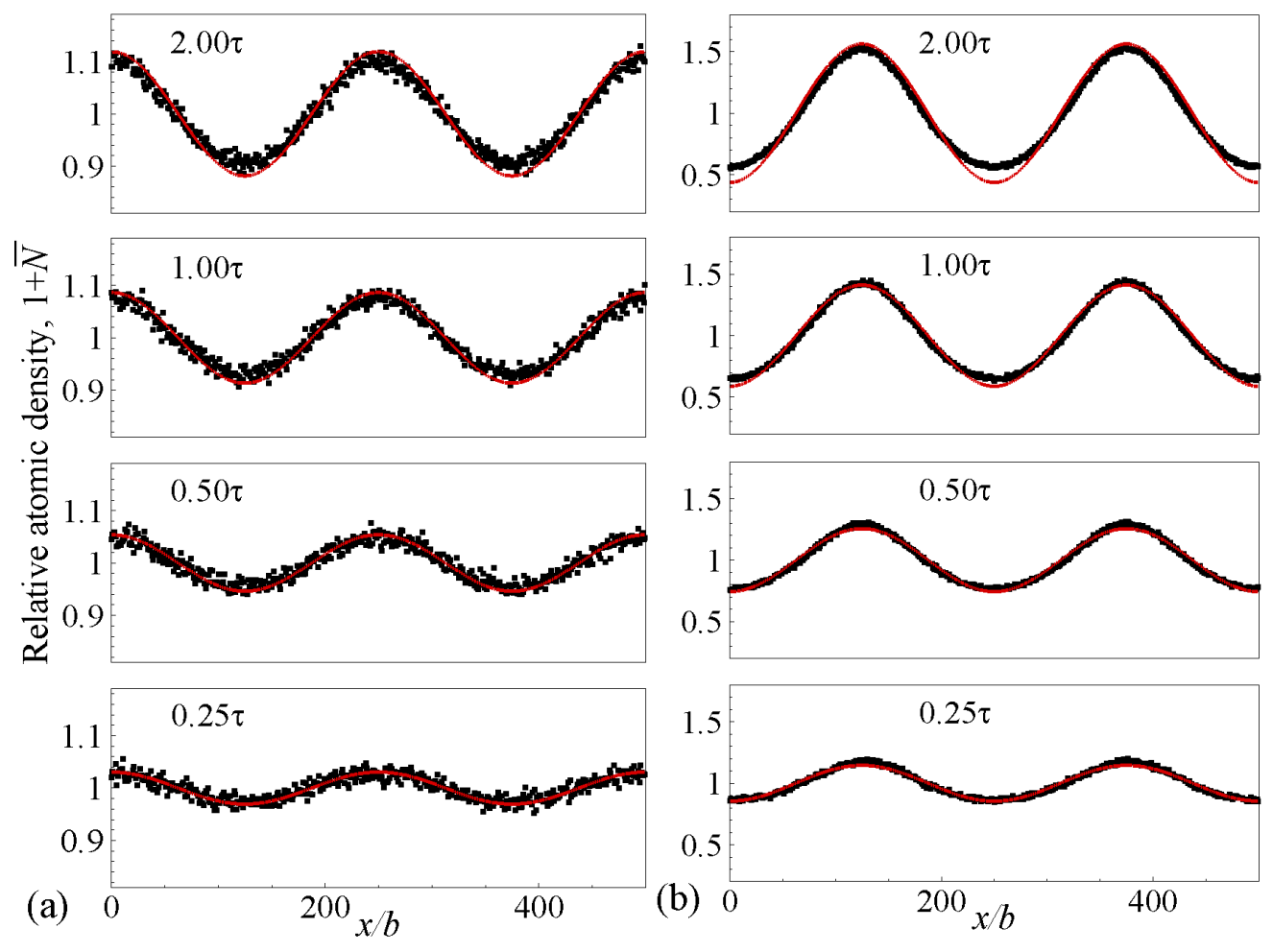

Figure 9.3: Examples of SAW-induced gathering of adatoms in the regions of SAW-strain nodes (a) and anti-nodes (b). Density profiles are shown for different times given in units of characteristic structuring time $\tau$. Red solid curves show predictions of Eq. (14) and black 
symbols are the results of kMC simulations. (a) is for a system with $\gamma=0.91 \mathrm{eV}, \delta=0.30 \mathrm{eV}, \theta=$ $1.49, E_{d}^{0}=0.66 \mathrm{eV}, k_{B} T_{0} / E_{d}^{0}=0.1\left(T_{0}=770 \mathrm{~K}\right)$, i.e., $\gamma_{\text {eff }}\left(\gamma_{\text {eff }}-\delta\right) /\left(E_{d}^{0}\right)^{2}=6.9$ and (b) is for a system with $\gamma=0.43 \mathrm{eV}, \delta=1.95 \mathrm{eV}, \theta=1.49, E_{d}^{0}=0.16 \mathrm{eV}, k_{B} T_{0} / E_{d}^{0}=0.1\left(T_{0}=190 \mathrm{~K}\right)$, i.e., $\gamma_{\text {eff }}\left(\gamma_{\text {eff }}-\delta\right) /\left(E_{d}^{0}\right)^{2}=-33.5$. The same strain magnitude $e_{0}=0.04$ is used in both simulations.

The feasibility of different structuring regimes in real material systems can be evaluated by considering the results of atomistic simulations of adatom diffusion on strained surfaces. In particular, recent simulations of adatom diffusion on an uniaxially strained (001) surface of a Lennard-Jones (LJ) crystal [166] reveal non-monotonous variations of $\gamma / E_{d}^{0}$ between 1.1 to 2.6 and $\delta / E_{d}^{0}$ between 0.5 to 12.0 as the size of the adatom changes from 0.8 to 1.5 of the size of atoms in the substrate. With $\gamma_{T} / E_{d}^{0}=1.5$ evaluated for the LJ substrate, these variations correspond to values of $\gamma_{\text {eff }}\left(\gamma_{\text {eff }}-\delta\right) /\left(E_{d}^{0}\right)^{2}$ ranging from -32.4 to 6.9 (Fig. 9.3 illustrates these two limiting cases). Even larger values of $\gamma(\gamma-\delta) /\left(E_{d}^{0}\right)^{2}$ can be obtained based on the results of $a b$ initio calculations performed for biaxially strained substrates, e.g., 539 for Ge/Ge(001) [146], -3 for $\mathrm{Ge} / \mathrm{Si}(001)$ [146], -14 for $\mathrm{In} / \mathrm{GaAs}(001)$ [144], and 299 for $\mathrm{Ag} / \mathrm{Ag}(111)$ [142].

The broad variability of the parameters that control surface structuring in different material systems indicates that different structuring regimes (gathering of adatoms in SAW-strain nodes or antinodes) may be realized for different substrate - adsorbate combinations. For an adsorbate that makes one successful jump per $10^{4}$ "attempts" (jump rate of $\sim 0.1-1 \mathrm{~ns}^{-1}$ ), $k_{B} T_{0}=E_{d}^{0} /(4 \ln 10)$, and the structuring contrast, $\sim\left[\gamma_{\text {eff }}\left(\gamma_{\text {eff }}-\delta\right) /\left(E_{d}^{0}\right)^{2}\right] \times 10 e_{0}^{2}$, can be sufficiently strong to induce acoustically-guided nucleation of 2D islands of adatoms.

Even stronger structuring can be achieved at extremely high frequencies of SAWs on the order of 100 s of $\mathrm{GHz}$, when the direct dynamic coupling between the acoustic wave and surface vibrations of adsorbates is possible. The results of recent MD simulations demonstrate that the dynamic (near-resonance) coupling is capable of strongly enhancing surface diffusion for running SAWs [166] and surface structuring for standing SAWs [161]. While the prospects for practical realization of the regime of dynamic coupling are boosted by recent advances in the development of photo-acoustic methods for generation of SAWs with frequencies approaching and exceeding $100 \mathrm{GHz}[124,125,140]$, strong attenuation and dispersion of high-frequency 
SAWs may limit utilization of this regime in applications. In contrast, the diffusional structuring regimes discussed in the present work have a relatively weak sensitivity to $\lambda$ (Fig. 9.2) and may be explored with conventional sources of SAWs.

\subsection{Conclusions}

Theoretical analysis of the phenomenon of structuring of surface adatoms in the presence of standing SAWs reveals a distinct diffusional mechanism that cannot be reduced to conventional paradigms based on analysis of inertial and radiation-induced average forces produced by oscillating inhomogeneous fields. The diffusional redistribution of surface adatoms is defined by a complex interplay between acoustically-induced transient modification of the diffusion activation barriers, local barrier asymmetry and adiabatic temperature variations. Explicit relationships between the SAW parameters and the effective "forces" controlling the characteristic time of diffusional structuring and its ultimate contrast are derived analytically and verified in $\mathrm{kMC}$ simulations. A rich variety of structuring regimes which are difficult to foresee intuitively, is established and related to the parameters of SAW and substrate-adatom interactions. Mapping the theoretical predictions to real material systems indicates that practical realization of surface structuring is feasible and suggests an attractive route for acoustic control of surface self-assembly without permanent modification of the substrate and growth conditions. 
Summary of the dissertation 


\section{Summary of the dissertation}

In Part I of this dissertation this dissertation, short (pico- to femtosecond) pulse laser interaction with metals are studied by performing MD simulations using the combined TTM-MD model. Rich information on the material response to the extreme conditions created by short pulse laser irradiation is obtained and, in particular, the microscopic mechanisms of the ultrafast structural and phase transformations in the irradiated targets are revealed. The connections between the simulation results and experimental observations are elucidated.

A special effort was devoted to the development of an efficient parallel code implementing the combined TTM-MD model using the Message Passing Interface (MPI) communication library. The code has demonstrated a very good parallelization scalability for simulations performed for systems of up to $\sim 340$ million atoms with the use of up to 24,000 computer cores. The efficient parallelization makes it possible to run large-scale simulations, which is often necessary in order to make direct connections to experimental observations.

An interesting phenomenon of generation of a runaway lattice-mismatched interface with complex three-dimensional corrugated structure is observed in the simulation of femtosecond laser irradiation of $\mathrm{Ag}-\mathrm{Cu}$ layered targets. The generation of this run-away lattice-mismatched interface can be attributed to the fast resolidification process under the conditions of strong undercooling created by short pulse laser irradiation. The complex three-dimensional corrugated structure of the interface is understood based on a detailed structural analysis, which identifies the dislocation configuration present at the interface. In addition, metastable $\mathrm{BCC} \mathrm{Cu}$ phase is found to form through epitaxial growth of $\mathrm{Cu}$ on FCC Ag (001) during resolidification process, which has been directly related to the experimental observations of pseudomorphic growth of BCC $\mathrm{Cu}$ during deposition of $\mathrm{Cu}$ on $\mathrm{Ag}$ (001) surface. Simulations of femtosecond laser irradiation of $\mathrm{Ag}$ film - $\mathrm{Cu}$ film - Ag (001) substrate system demonstrate that the BCC $\mathrm{Cu}$ can be formed and stabilized when sandwiched between two FCC Ag (001)/BCC Cu (001) interfaces, which suggests a possible way of generation of "stable" BCC $\mathrm{Cu}$ by atomic depositions of $\mathrm{Ag}-$ $\mathrm{Cu}-\mathrm{Ag}$ multilayer films. Finally, a much wider (5 times wider) atomic mixing region compared to the width of the equilibrium interface in this immiscible $\mathrm{Ag}-\mathrm{Cu}$ system is observed in simulations of short pulse laser irradiation of $\mathrm{Ag}-\mathrm{Cu}$ systems. 
Simulations of short pulse laser irradiation of $\mathrm{Ag}$ targets are performed, and it is found that the short pulse laser-induced photomechanical processes lead to the voids nucleation, growth and coalescence. At laser fluence just below the spallation threshold, the voids can be captured by fast resolidification. The captured voids increase the volume of the surface region, leading to an effective "swelling" of the irradiated spot. The computational prediction of the generation of porous structure of a surface region of the irradiated target provides an explanation of the recently reported experimental observations of surface swelling, or "frustrated ablation," in Al and Ag targets irradiated by 100 fs laser pulses. In addition, the large-scale simulations of short pulse laser irradiation of Ag targets have revealed generation of a $\sim 30 \mathrm{~nm}$ surface layer of nanocrystalline structure. The generation of the nanocrystalline structure can be attributed to the homogeneous nucleation and growth of multiple randomly oriented crystallites under the condition of strong undercooling that is achieved during the short pulse laser irradiation. The atomistic picture of the homogeneous nucleation is explored. The structural analysis on the generated nanocrystalline structure shows that all nanograins have close-packed structure, with large number of stacking faults and twin boundaries. More interestingly, the complicated fivefold twinning structures are formed. The grain boundaries, stacking faults, and twin boundaries are all likely to present strong barriers for dislocation propagation, resulting in the effective hardening of the surface region of the irradiated target.

In Part II of this dissertation, atomic-level simulations (MD and kMC) are combined with theoretical analysis of the effect of laser-induced surface acoustic waves on surface diffusion. It is found that a substantial diffusion enhancement (up to tens and hundreds of percents) by laserinduced surface acoustic waves is possible via (1) transient surface strain-induced modification of the diffusion barriers, (2) adiabatic variation in the surface temperature, and (3) dynamic coupling of the acoustic waves with vibrational states of adsorbed species. The approximate scaling laws describing the first two effects are established and verified in kinetic Monte-Carlo simulations. The combined contribution of all three effects is studied in molecular dynamics simulations and the conditions for the diffusion activation through the dynamic coupling are elucidated. The acoustic enhancement of surface diffusion provides an attractive alternative to thermal activation in thin film growth on heat-sensitive substrates.

In addition to the surface diffusion enhancement, it is found that conditions for effect the self-structuring (spatial modulation of adatom concentration) can be created by the laser-induced 
standing surface acoustic waves. The mechanism of the self-structuring is explored theoretically and in kinetic Monte Carlo (kMC) simulations. The results of this exploration reveal several distinct structuring regimes and directly relate them to the transient modification of diffusion barriers and adiabatic temperature variations induced by SAW strains. The predicted phenomenon indicates that practical realization of surface structuring is feasible and suggests an attractive route for acoustic control of surface self-assembly without permanent modification of the substrate and growth conditions. 
Appendix 


\section{Appendix A: MPI parallelization of the combined TTM-MD model}

\subsection{Brief review of the combined TTM-MD model}

Before discussing the details of the MPI parallelization of TTM-MD model [34], the basic idea of the TTM-MD model is reviewed. Briefly, the model combines the classical molecular dynamics (MD) method for simulation of non-equilibrium processes of lattice superheating and fast phase transformations induced by the laser energy deposition with a continuum description of the laser excitation and subsequent relaxation of the conduction band electrons, based on the so-called two-temperature model (TTM) [49]. As schematically illustrated in Fig. 11.1, the MD method is combined with TTM equation for $T_{e}$ in the very surface region of the target, in which MD substitutes the TTM equation for $T_{l}$ to capture the active processes of laser melting, void nucleation, and spallation/ablation, whereas TTM equation for $T_{e}$ accounts for the dominant electronic thermal conduction. The diffusion equations for both electron $T_{e}$ and lattice $T_{l}$ temperature are solved in the deeper region affected by the thermal conduction. A special pressure transmitting boundary condition [51,52] is used in this case to imitate the propagation of the laser induced stress wave from the MD region of the computational system to the continuum part of the model. The energy carried away by the stress wave is monitored to check energy conservation in the combined atomistic-continuum model [53].

The diffusion equation for electronic temperature $T_{e}$ (\& lattice temperature $T_{e}$ in deeper part) is solved by a finite difference method simultaneously with MD integration of the equations of motion of atoms. The cells in the finite difference discretization are related to the corresponding volumes of the MD system and the local lattice temperature $T_{1}$. is calculated for each cell from the average kinetic energy of thermal motion of atoms. An additional force, $\xi m_{i} \vec{v}_{i}^{\text {th }}$, is added to the MD equations of motion to account for the electron-phonon coupling. In this coupling term, $m_{i}$ is the mass of an atom, $\vec{v}_{i}^{\text {th }}$ is the thermal velocity of an atom defined as $\vec{v}_{i}^{t h}=\vec{v}_{i}-\vec{v}_{i}^{c}$, where $\vec{v}_{i}$ is the actual velocity of an atom $i$ and $\vec{v}_{i}^{c}$ is the velocity of the center of mass of a cell to which atom $i$ belongs, and $\xi$ is a coefficient defined as $\xi=\frac{G V_{N}\left(T_{e}-T_{l}\right)}{2 K^{T}}$, where 
$G$ is electron-phonon coupling factor, $V_{N}$ is the cell volume, and $K^{T}$ is the thermal kinetic energy of the cell volume. The expansion, density variations and disintegration of the irradiated target predicted in the MD part of the model are accounted for in solving the equation for the electronic temperature $T_{e}$ evolution. The 3-dimensional solution of the diffusion equation for $T_{e}$ is used in simulations of laser ablation or spallation, where the development of large density variations at the initial stage of the ablation process or voids in spallation can affect the lateral heat conduction in the target.

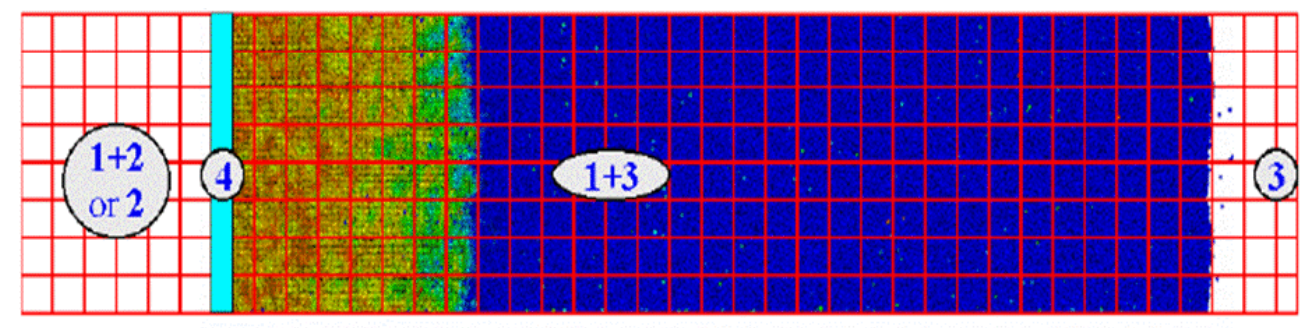
(1) $C_{e}\left(T_{e}\right) \frac{\partial T_{e}}{\partial t}=\nabla \cdot\left[K_{e}\left(T_{e}, T_{i}\right) \nabla T_{e}\right]-G\left(T_{e}\right)\left(T_{e}-T_{i}\right)+S(\vec{r}, t)$
(2) $C_{l}\left(T_{l}\right) \frac{\partial T_{l}}{\partial t}=\nabla \cdot\left[K_{l}\left(T_{l}\right) \nabla T_{l}\right]+G\left(T_{e}\right)\left(T_{e}-T_{l}\right)$
TTM
(3) $m_{i} d^{2} \vec{r}_{i} / d t^{2}=\vec{F}_{i}+\xi m_{i} \vec{v}_{i}^{t h}, \quad T_{i}^{\text {cell }}=\sum_{\text {cell }} m_{i}\left(v_{i}\right)^{t / h} /\left(3 k_{B} N_{c e l l}\right)$
MD
(4) pressure-transmitting, heat-conducting boundary conditions
$\xi=\frac{G V_{N}\left(T_{e}-T_{l}\right)}{2 K^{T}}$

Figure 11.1: Schematics of the TTM-MD model for simulation of laser interaction with a metal target. The laser irradiation is directed from the right side.

\subsection{MPI parallelization of the combined TTM-MD model}

The combined TTM-MD model has been demonstrated to be suitable for simulating the short-pulse laser-metal interactions. The need to directly compare simulation results with experiments often requires large scale simulations on big systems that consist of $~ 100$ million atoms. It is impossible to run these large scale simulations using one computer core with a serial implementation of the TTM-MD model due to two main reasons. Firstly, the operational memory of one computer core is limited, and there is not enough memory even for storing atomic coordinates and velocities. Secondly, the speed of the calculation is extremely slow for 
these large scale simulations. Currently, simulations with the serial MD code are limited to systems with less than one million atoms. To enable large-scale simulations, the parallelization of the combined TTM-MD model is needed.

The basic idea of the parallelization is to divide a "big" calculation into many "small" ones, which are executed by many computer cores in parallel. By doing this, the calculation speed is increased due to the small calculation load for each core. On the other hand, the total size of the simulation system can be increased dramatically, since each core only needs to store information about a small part of the system. Different types of parallelization can be distinguished based on whether the information exchange (communication) between the computer cores is needed or not. Perfect parallelization can be achieved if no communication is needed during the whole calculation process, i.e., the calculations are independent among computer cores. The calculation speed increases proportionally with number of computer cores used for this perfect parallelization, i.e., the perfect parallelization scalability is achieved. In most cases, particularly in the classical MD or the combined TTM-MD, the communication among computer cores is required during calculations. To achieve good parallelization scalability for these cases, one has to carefully implement the parallel algorithm.

In this work, we apply the Message Passing Interface (MPI) communication library for parallelization of the TTM-MD code, written in Fortran 90. We begin with breaking calculations involved in the TTM-MD model into two general types: integration of the Newton equations for atomic motions in the MD part of the model and solving the equations for the electron and lattice temperature evolution in the TTM part of the model. Accordingly, the requested computer cores are divided into 2 parts: one (main) part of the computer cores, called MD cores, are distributed for MD integration, and the other part of the computer cores, called TTM cores, are distributed for solving TTM equation, as illustrated in Fig. 11.2. Then we divide the MD system into a number of MD subdomains, with each MD subdomain assigned to a single MD core. Similarly, the TTM system is divided into larger TTM subdomains, with each TTM subdomain assigned to a single TTM core. For the convenience, the MD cores are grouped into a separate "communicator", so called "MD_COMM_WORLD", and the TTM cores are grouped into the communicator of "TTM_COMM_WORLD". As shown in Fig. 11.2, the two types of parallelization discussed above, parallelization of MD integration of atomic motions and parallelization of solving TTM equations, require three types of communications: 1) 
communication among MD cores based on communicator "MD_COMM_WORLD", 2) communication among TTM cores based on communicator "TTM_COMM_WORLD”, 3) communication between the MD cores and TTM cores based on a common default communicator "MPI_COMM_WORLD”. Below, we go over the MPI parallelization details in 3 steps. Firstly, the MPI parallelization of MD integration (including the internal communication among MD cores) is discussed. Then, we turn to the MPI parallelization of TTM part (including the internal communication among TTM cores). At last, we discuss the combination of the MD parallelization with TTM parallelization and the communication between the MD cores and TTM cores.

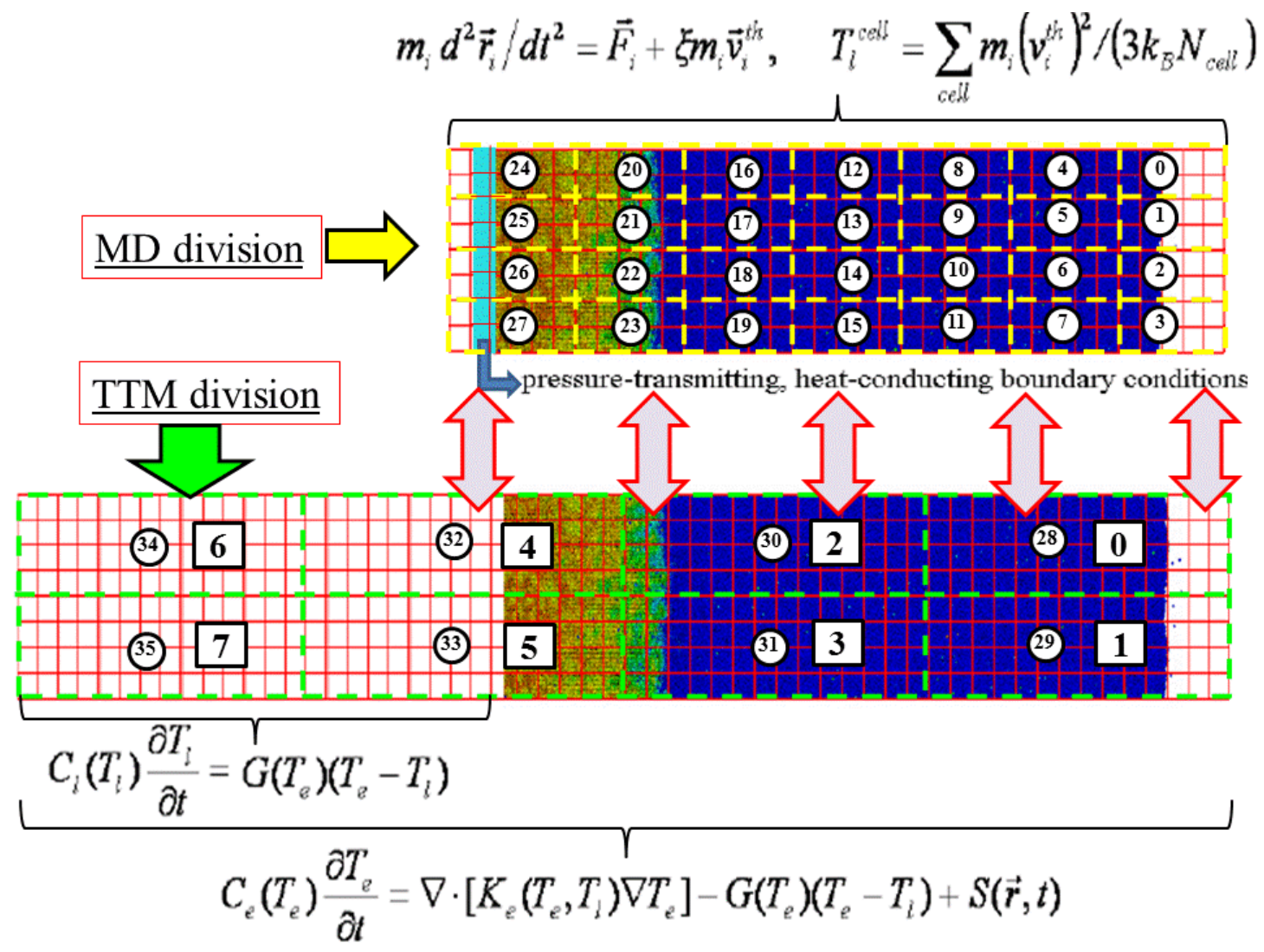

Figure 11.2: Schematics of the parallelization of the TTM-MD model for simulation of laser interaction with a metal target. The laser irradiation is directed from the right side. As an example, here the MD system is divided into 28 subdomains and the TTM system is divided into 8 subdomains. Each subdomain corresponds to a single computer core, and the total number of requested computer cores is 36 . Out of the total 36 cores, 28 (0-27) cores, grouped into "MD_COMM_WORLD", are used for MD integration and the remaining 8 (28-35) cores 
(“TTM_COMM_WORLD") are used to solve TTM equations. The red squares are the cells used for solving the TTM equations with a finite difference method. The red arrows indicate the communication between MD cores and TTM cores.

\subsubsection{MPI parallelization of the MD part}

In order to parallelize the MD part, we break the MD system down to a number of subdomains as shown in Fig. 11.3, each corresponding to a single computer core with a given "global" ID in "MD_COMM_WORLD", which is responsible for the MD integration of atomic motions in this subdomain. The "global" ID can be derived from its component ID in each direction. For example, if the system is divided into NXD subdomains in $x$ direction, NYD subdomains in y direction and NZD in z direction, the global ID GID of a subdomain can be obtained from its $\mathrm{x}$ component ID XID, $\mathrm{y}$ component ID YID and $\mathrm{z}$ component ID ZID as follows: $G I D=X I D+N X D \times(Y I D+N Y D \times Z I D)$. With this numbering convention, each subdomain can easily identify its neighboring subdomains for communication.

\subsubsection{Linked list, neighbor list and MD communication}

As shown in Fig. 11.3, each MD subdomain is further divided into link cells (white squares), with the size a little greater than the cutoff distance of interatomic interaction potential plus a buffer distance ( $1 \AA$ ). A single-link-cell border layer (green slashes) and a single-link-cell skin layer (purple slashes) are identified for each subdomain and shown for the subdomain 10 as an example. It is easily seen that the border layer of the current subdomain is the skin layer of its corresponding neighboring subdomains and the skin layer of the current subdomain is the border layer of its corresponding neighboring subdomains. The information of atoms in the border layer is sent to the corresponding neighboring subdomains. By this communication, the information of atoms of the skin layers can be updated. Atoms in each link cell are linked in a chain, so called "linked list" [170,171]. In order to generate the linked list, two arrays are used: one saves the head of the chain, i.e., the ID of the first atom in the list, for each link cell, and the other saves a sequence of IDs of all atoms in the chain. The end of the chain is reached if the ID of the next atom is zero.

Taking advantage of the linked list algorithm [170,171], we construct the neighbor list of each atom by firstly going through the linked list of the current link cell and then 26 neighboring link cells. Due to the fact that if atom A is a neighbor of atom B, the atom B must also be a 
neighbor of atom A, we can further improve the efficiency of neighbor list generation by only going through 13 neighboring link cells. To save the computational time (the atom's neighbor list generation is time consuming and takes $\sim 50 \%$ of the total computational time), we renew the linked list and neighbor list every 10 time steps (10 fs), by generating a little "larger" neighbor list (the atoms are counted as neighbors within the range of interaction cutoff distance plus a buffer zone with the size of $1 \AA$ ) to account for the atomic displacements during 10 time steps (We assume here that the relative atomic displacements during the time of $10 \mathrm{fs}$ cannot exceed 1 $\AA$, i.e., the relative atomic velocity of any pair of atoms cannot be higher than $100 \AA / \mathrm{ps}$ or $10^{4}$ $\mathrm{m} / \mathrm{s}$ ). In addition, the "migration" process is performed every 10 time steps to send atoms that move outside of the current subdomain to its correct subdomains and receive atoms that move into the current subdomain from its neighboring subdomains. The information on atomic positions of atoms in the skin cells are updated by communication every time step to ensure the correct MD integration.

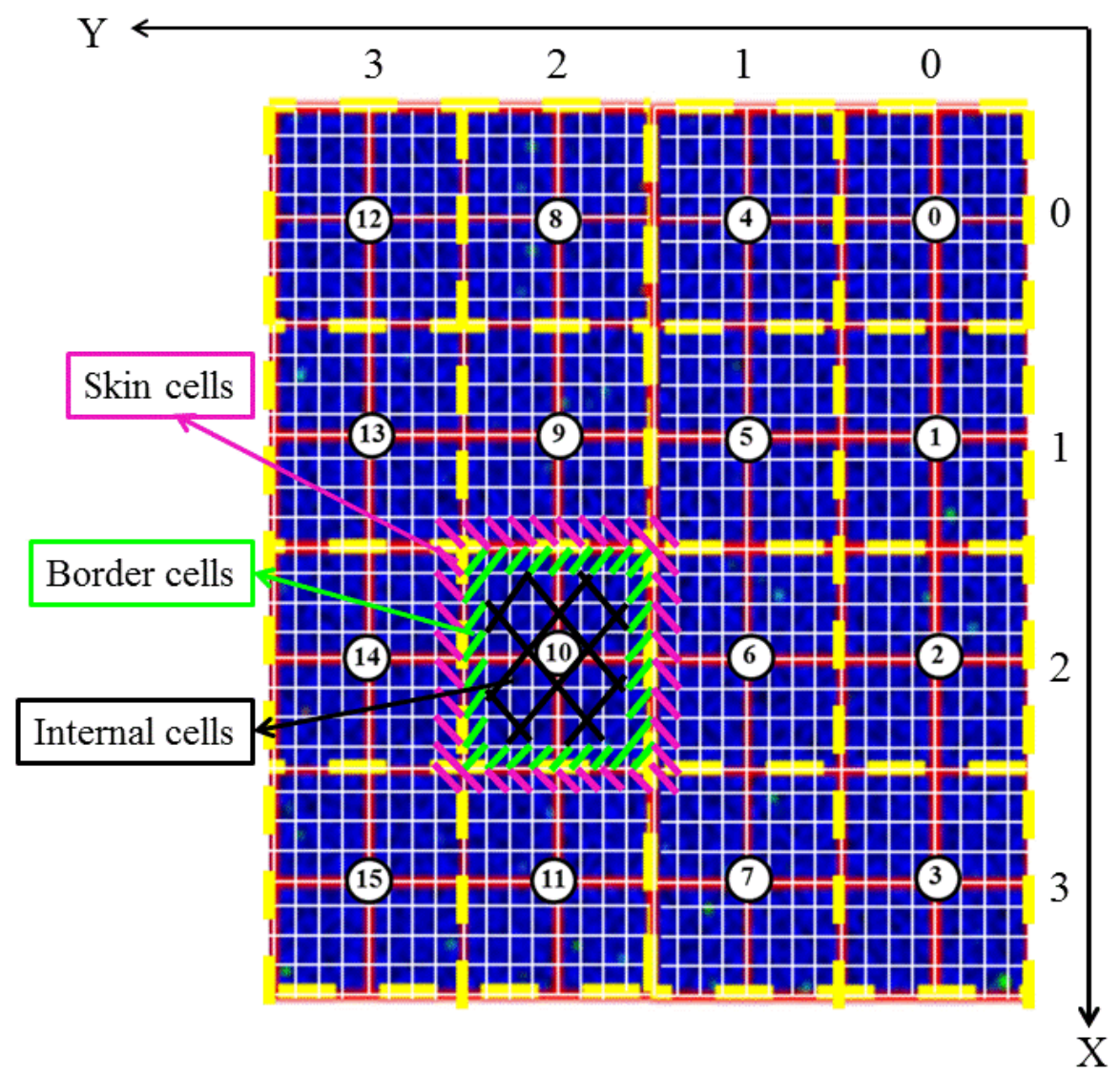

Figure 11.3: Spatial division of the MD computational cell into subdomains for parallelization. Each subdomain corresponds to a single computer core. Each subdomain is further divided into 
MD link cells (white squares). The TTM cell (red squares) used to calculate lattice temperature (for TTM) has the size of multiple times of a MD link cell. The division ensures an integer number of "TTM" cells in each subdomain. A single-link-cell border layer (green slashes) and a single-link-cell skin layer (purple slashes) are identified for each subdomain for the convenience of communications with neighboring subdomains and are shown for the subdomain 10 as an example.

\subsubsection{MD integrator}

The Nordsieck fifth-order predictor-corrector algorithm [170] is applied for MD integration. This algorithm is one of the most efficient integrators that ensures a good accuracy. Assume that an atom has coordinate $r(t)$ and its time derivatives are $q_{1}(t)=h \frac{d r}{d t}, q_{2}(t)=\frac{h^{2}}{2} \frac{d^{2} r}{d t^{2}}, q_{3}(t)=\frac{h^{3}}{6} \frac{d^{3} r}{d t^{3}}, q_{4}(t)=\frac{h^{4}}{24} \frac{d^{4} r}{d t^{4}}, q_{5}(t)=\frac{h^{5}}{120} \frac{d^{5} r}{d t^{5}}$, where $h$ is the time step of MD integration. The Nordsieck algorithm can be described as a 2-step procedure.

Step 1 (prediction, Nord5_Step1): new values of coordinates and their time derivatives are obtained through corresponding Taylor series, without any account of interatomic interactions, i.e., the atoms are moving "by interia".

$r^{p}(t+h)=r(t)+q_{1}(t)+q_{2}(t)+q_{3}(t)+q_{4}(t)+q_{5}(t)$

$q_{1}^{p}(t+h)=q_{1}(t)+2 q_{2}(t)+3 q_{3}(t)+4 q_{4}(t)+5 q_{5}(t)$

$q_{2}^{p}(t+h)=q_{2}(t)+3 q_{3}(t)+6 q_{4}(t)+15 q_{5}(t)$

$q_{3}^{p}(t+h)=q_{3}(t)+4 q_{4}(t)+10 q_{5}(t)$

$q_{4}^{p}(t+h)=q_{4}(t)+5 q_{5}(t)$

$q_{5}^{p}(t+h)=q_{5}(t)$

Step 2 (correction, Nord5_Step2): the atomic coordinates, velocities and higher order derivatives are corrected based on the force calculation. If we define $\delta q_{2}(t+h)=\frac{F(t+h)}{2 m} h^{2}-q_{2}{ }^{p}(t+h)$, the corrections can be performed as shown below. 


$$
\begin{array}{ll}
r(t+h)=r^{p}(t+h)+C_{0} \delta q_{2}(t+h) & C_{0}=\frac{3}{20} \\
q_{1}(t+h)=q_{1}{ }^{p}(t+h)+C_{1} \delta q_{2}(t+h) & C_{1}=\frac{251}{360} \\
q_{2}(t+h)=q_{2}{ }^{p}(t+h)+C_{2} \delta q_{2}(t+h) & C_{2}=1 \\
q_{3}(t+h)=q_{3}{ }^{p}(t+h)+C_{3} \delta q_{2}(t+h) & C_{3}=\frac{11}{18} \\
q_{4}(t+h)=q_{4}{ }^{p}(t+h)+C_{4} \delta q_{2}(t+h) & C_{4}=\frac{1}{6} \\
q_{5}(t+h)=q_{5}{ }^{p}(t+h)+C_{5} \delta q_{2}(t+h) & C_{5}=\frac{1}{60}
\end{array}
$$

\subsubsection{Implementation of MD integrator: computation and communication overlap}

One way to design an efficient parallelization algorithm is to allow for an overlap between communication and computation, i.e., perform communication in parallel with computation. To achieve this, a specific algorithm for implementation of Nordsieck fifth-order predictor-corrector MD integrator is developed as shown in Fig. 11.4 and discussed below. Here we choose metal system with EAM potential [59] as an example. The basic idea of EAM potential is that it defines the total energy of a system of interacting atoms as the total energy of pair interactions, plus an addition energy term that depends on the local "electron density" $\rho$ and is called the embedding energy. To calculate the force of the atom, we need to know the coordinates and also the "electron densities" of the current atom and its neighboring atoms. A detailed description of EAM potential is provided in section 2.4. 


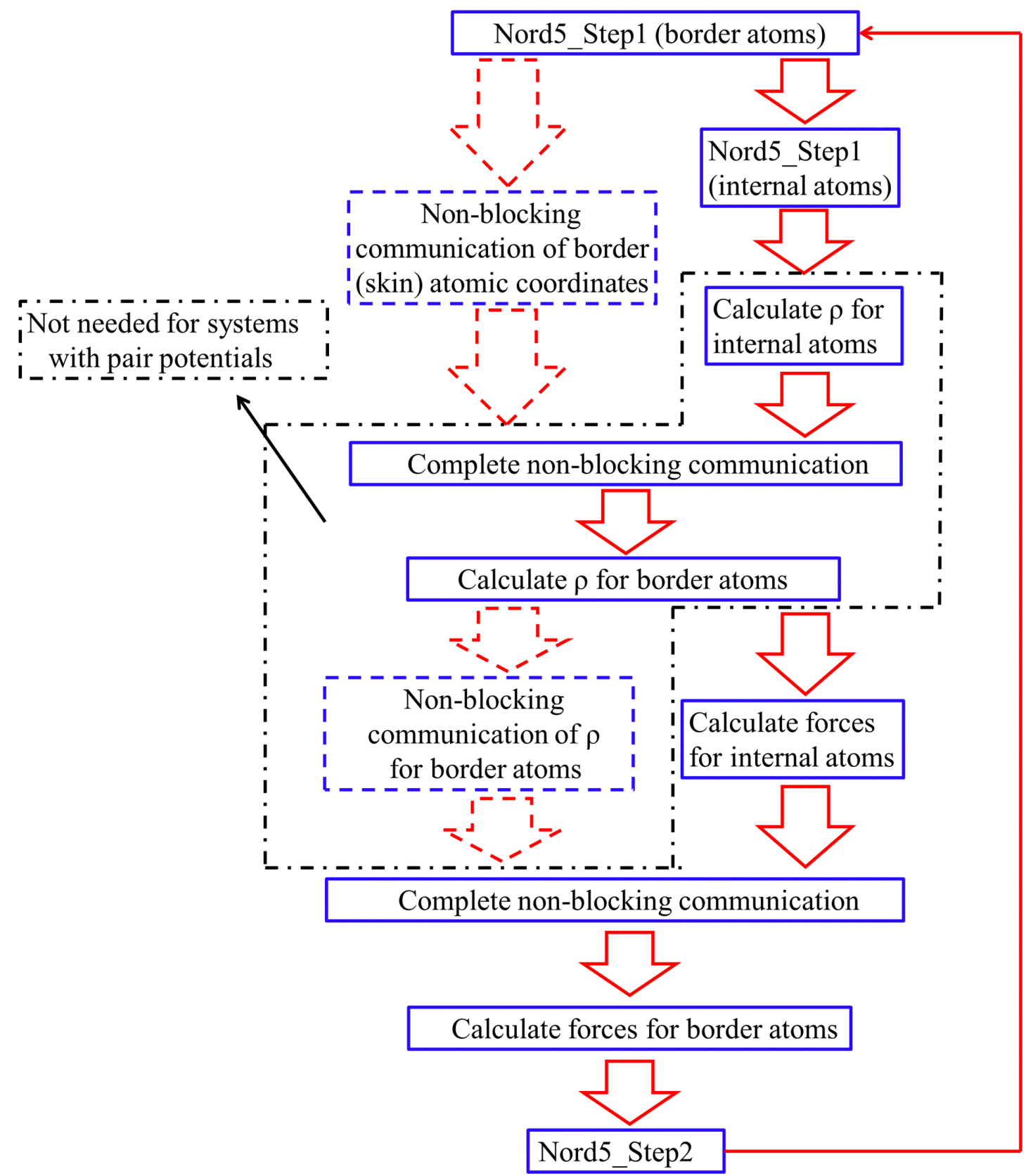

Figure 11.4: Algorithm for implementation of Nordsieck fifth-order predictor-corrector MD integrator to achieve MPI parallelization with the overlap of communication and computation for a system with EAM potential. For the systems with pair potentials (e.g., L-J), the steps outlined by the black dot dashed lines are not needed.

The steps involved in the parallel integration of the MD equations of atomic motions as illustrated in Fig. 11.4 are as follows.

Step 1: Perform Nord5_Step1 for the atoms in border cells. This calculation doesn't require any 
information of neighboring atoms.

Step 2: Initiate "non-blocking" communication to send (MPI_ISEND) the newly calculated (step 1) coordinates of atoms in the border cells to (the skin cells of) the corresponding neighboring subdomains. At the same time, initiate "non-blocking" communication to receive (MPI_IRECV) the newly calculated coordinates of atoms in the skin cells from the corresponding neighboring subdomains. Using "non-blocking" communications (send and receive), the computer cores process the communications in background and continue with the computations of Step 3. In other words, "non-blocking" communications allow the overlap of computation and communication.

Step 3: Perform Nord5_Step1 for the rest of atoms in the internal cells of the current subdomain. During this computation, the "non-blocking" communication initiated at Step 2 is processed in the background.

Step 4: Calculate the local "electron density" for the atoms in the internal cells of the current subdomain, $\rho_{\mathrm{i}}=\sum_{j \neq i} f_{j}\left(r_{i j}\right)$, with the updated atomic coordinates obtained in Step 1 and 3. This does not require the updated coordinates of atoms in skin cells, since they cannot be the neighbors of atoms in the inside cells. During this computation, the "non-blocking" communication initiated at Step 2 is still processed in the background.

Step 5: "Wait" for the Step 2-initiated communication to complete and update the coordinates of atoms in the skin cells of the current subdomain with the received message.

Step 6: Calculate the local "electron density" for the atoms in the border cells of the current subdomain with the updated coordinates, $\rho_{\mathrm{i}}=\sum_{j \neq i} f_{j}\left(r_{i j}\right)$.

Step 7: Initiate "non-blocking" communication to send (MPI_ISEND) the newly calculated (step 6) "electron densities" of atoms in the border cells to (the skin cells of) the corresponding neighboring subdomains. At the same time, initiate "non-blocking" communication to receive (MPI_IRECV) the newly calculated "electron densities" of atoms in the skin cells from the corresponding neighboring subdomains. The "Non-blocking" communications allow the overlap of computation and communication.

Step 8: Calculate the force acting on the atoms in the internal cells of the current subdomain with 
the updated atomic coordinates and "electron densities". This does not require the updated "electron densities" of atoms in skin cells, since they cannot be the neighbors of atoms in the internal cells. During this computation, the "non-blocking" communication initiated at Step 7 is processed in the background.

Step 9: "Wait" for the Step 7-initiated communication to complete and update the "electron density" of atoms in the skin cells of the current subdomain with the received message.

Step 10: Calculate the force acting on the atoms in the border cells of the current subdomain with the updated atomic coordinates and "electron densities". If non-reflecting boundary condition is used, extra force is calculated for the atoms in the boundary region. In the case of TTM-MD simulation of laser-metal interaction, the additional force due to electron-phonon coupling is also calculated at this step.

Step 11: Perform Nord5_Step2 to update coordinates, velocities and higher order derivatives for all atoms in the current subdomain and go back to Step 1.

\subsubsection{TTM cells for TTM-MD simulation}

In the case of simulations of laser-metal interaction with TTM-MD model, so called "TTM cells" (red squares as in Figs. 11.2 and 11.3) are also defined in the each MD subdomain. The lattice temperature $T_{l}$ is calculated for each TTM cell as an input to solve the TTM equation for the electronic temperature $T_{e}$. To ensure reliable calculation of the lattice temperature, each TTM cell needs to have $\sim 300$ atoms before laser irradiation. As discussed above in section 11.2.1.1, each MD link cell has the size a little larger than the interaction cutoff distance plus the 1 A buffer to ensure efficient neighbor list generation and MD communication. For example, the MD link cell for the FBD EAM Ag system has the size $\sim 7 \AA$, consisting of $\sim 22$ atoms. Thus, the TTM cell should be much larger than the MD link cell. To simplify the algorithm, we choose the TTM cell size to be multiple of the size of the MD link cell as shown in Fig. 11.3. On the other hand, strong electronic temperature gradient is generated along z-direction during laser irradiation, which requires small step in the spatial discretization in the solution of the TTM equation for the electronic temperature. So the TTM cell is chosen to coincide with the MD link cell along z-direction, i.e., TTM cell has the same size as MD cell along z-direction. From my experience, $1 \mathrm{~nm}$ is a good choice for the size in z-direction for both MD link cells and TTM cells. In $\mathrm{x}$ - and $\mathrm{y}$ - directions, the TTM cells can be chosen to be 4 times larger than the MD cells 
as shown in Fig. 11.3.

\subsubsection{MPI parallelization of the TTM part}

To solve the TTM equation for the electronic temperature $T_{e}$, $C_{e}\left(T_{e}\right) \frac{\partial T_{e}}{\partial t}=\nabla \cdot\left(K_{e}\left(T_{e}, T_{l}\right) \nabla T_{e}\right)-G\left(T_{e}\right)\left(T_{e}-T_{l}\right)+S(z, t)$, the TTM system is divided into TTM cells (red squares) as shown in Fig. 11.2. Similarly to the MD parallelization, we break the TTM system down to a number of subdomains, with each TTM subdomain corresponding to a single TTM computer core with a given "global" ID in "TTM_COMM_WORLD". This TTM core is responsible for solving the TTM equations of TTM cells in the subdomain. The "global" ID can be derived from its component IDs in each direction in the same way as for the MD part. The finite difference method is used to solve the TTM equations. The stability criterion $\frac{D \Delta t}{\Delta x^{2}}+\frac{D \Delta t}{\Delta y^{2}}+\frac{D \Delta t}{\Delta z^{2}} \leq \frac{1}{2}$ needs to be satisfied when we choose the time step for solving this diffusion equation, where $D=K_{e}\left(T_{e}, T_{l}\right) / C_{e}\left(T_{e}\right)$. Three energy channels are clearly seen from the TTM equation. The first one is the heat conduction between the neighboring TTM cells, $\nabla \cdot\left(K_{e}\left(T_{e}, T_{l}\right) \nabla T_{e}\right)$. In the case of 3D TTM-MD model, each TTM cell exchanges energy by heat conduction with 6 neighboring TTM cells ( 2 in each direction). The second one is the electronphonon coupling, $-G\left(T_{e}\right)\left(T_{e}-T_{l}\right)$ in the current TTM cell. The energy is transferring from the electronic system to the lattice system, when the electronic temperature is higher than the lattice temperature. The third channel of the energy input is from the laser energy deposition $S(z, t)$. The effect of atomic density variations in the MD part of the model on the parameters of TTM equations $\left(C_{e}, K_{e}\right.$ and $\left.G\right)$ is taken into account in solving the TTM equation. Similarly to the parallelization of the MD part, a single-TTM-cell border layer and a single-TTM-cell skin layer are identified for each TTM subdomain. The information of electronic temperature in the skin cells needs to be updated by receiving message from the neighboring subdomain every TTM diffusion time step. An approach similar to the one discussed in section 11.2.1.3 is applied to achieve the parallelization with overlap of communication and computation as shown in Fig. 11.5. 


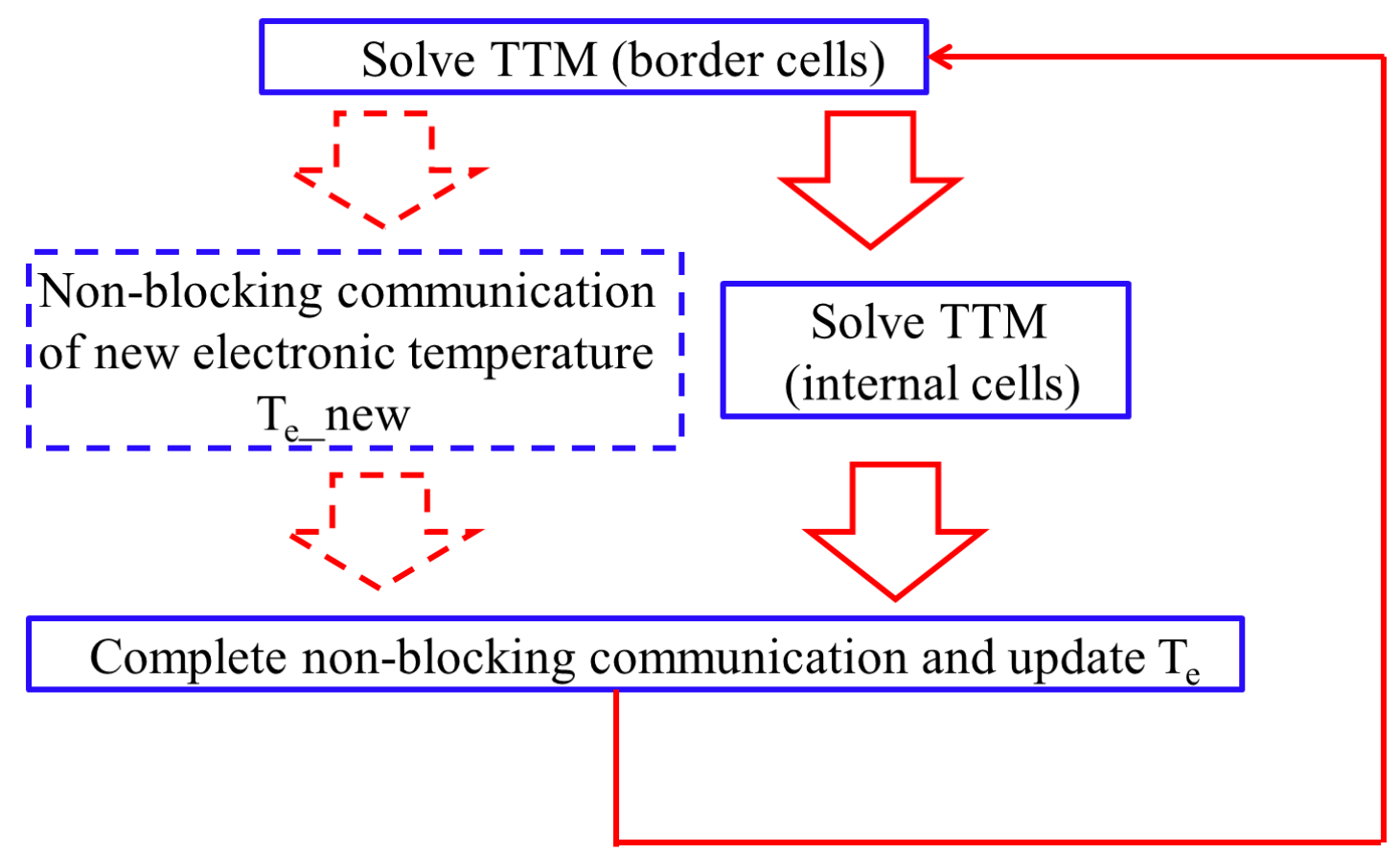

Figure 11.5: Algorithm to achieve MPI parallelization of TTM part with the overlap of communication and computation.

\subsubsection{Combination of TTM parallelization and MD parallelization}

Even though we divide the computer cores into two parts, one part for MD parallelization and the other part for TTM parallelization, they are not independent of each other. The combined TTM-MD model involves communication between TTM and MD computer cores. On one hand, the MD cores send the newly calculated lattice temperature $T_{l}$, and atomic density to the corresponding TTM cores. On the other hand, the TTM cores send the newly calculated electron-phonon coupling energy to MD cores in order to calculate the additional forces (due to e-ph coupling) in the MD part. Actually, in the simulations, we have to order the TTM and MD calculations in a way that is shown in Fig. 11.6. For example, at a MD time step $N$, the MD calculations are performed using the value of $\xi$ calculated with e-ph coupling energy obtained from TTM calculations at time step $N-1$. Then the newly calculated lattice temperature is sent to TTM cores for their calculation of the step $N$. To make the MD and TTM "trains" move fluently, computer cores are distributed wisely between the TTM and MD parts so that the work load for the TTM and MD cores is similar and it takes similar CPU time per MD time step for both TTM and MD cores. 


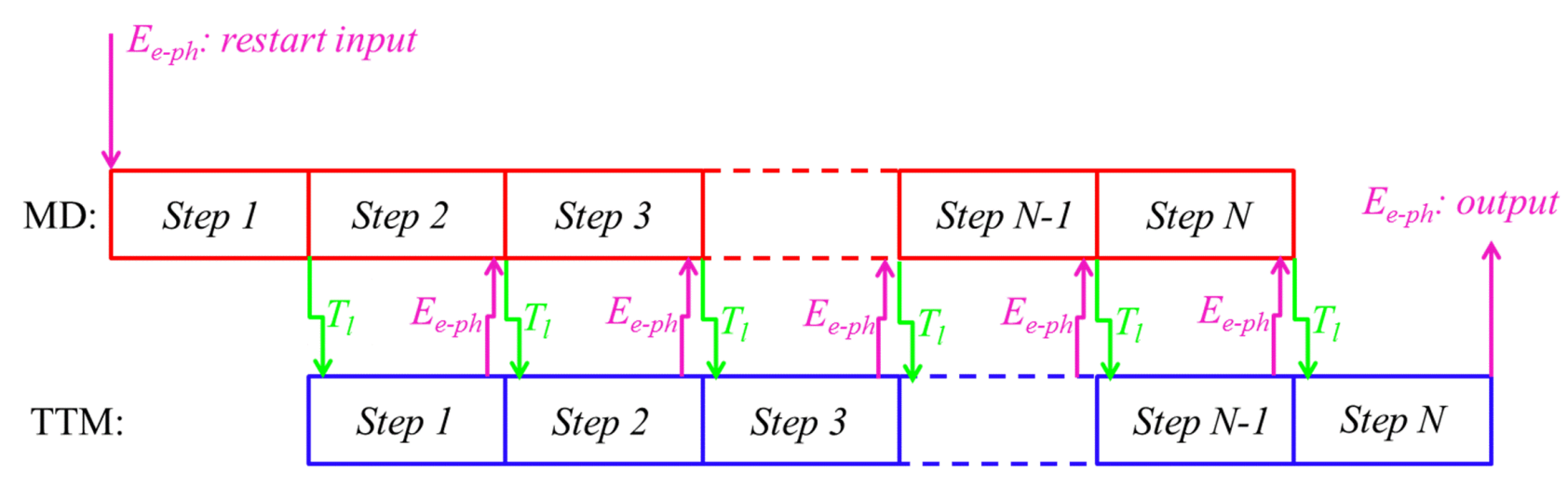

Figure 11.6: Communication between MD and TTM cores used for synchronization of the TTM and MD parts of the model.

\subsubsection{General structure of the parallel algorithm for the TTM-MD model}

Finally, we are ready to discuss the general structure of the parallel algorithm implementing the TTM-MD model as shown in Fig. 11.7. To save the operational memory and make the code more flexible, the memory is allocated for each core dynamically. To make sure the simulation runs efficiently, the code keeps checking the load balancing between the cores, if some of the cores are overloaded, the simulation writes the restart files and stops for the redividing the load between the cores.

The parallel algorithm implementing the combined TTM-MD model can be described as sequence of steps listed below. The corresponding names of Fortran files are also provided where appropriate.

1) The core " 0 " reads all the common input files (md.input, etc) and broadcasts the data to the other cores. $\rightarrow$ ReadFiles. $f 90$

2) Cores are divided into two categories: MD and TTM cores. MD cores are grouped as a communicator "MD_COMM_WORLD" and TTM cores are grouped as a communicator "TTM_COMM_WORLD".

3) A: Each MD core defines its own geometry (subdomain size and boundary). $\rightarrow$ SetNodeMD.f90

B: Each TTM core defines its own geometry (subdomain size and boundary). $\rightarrow$ SetNodeTTM.f90

4) A: Each MD core allocates its own memory that is required for simulation. $\rightarrow$ 
Allocate_MD.f90

B: Each TTM core allocates its own memory that is required for simulation. $\rightarrow$ Allocate_TTM.f90

5) A: Each MD core finds the corresponding TTM cores that it needs to communicate with. $\rightarrow$ MDRecvTTM.f90

B: Each TTM core finds the corresponding MD cores that it needs to communicate with. $\rightarrow$ TTMRecvMD.f90

6) A: Each MD core reads its own input of atomic coordinates, velocities, etc. $\rightarrow$ ReadDataMD. 990

B: Each TTM core reads its own input of electronic temperature, lattice temperature, etc. $\rightarrow$

\section{ReadDataTTM.f90}

7) Energy and Force tables are generated for MD cores. $\rightarrow \boldsymbol{E f t a b}-* . f 90$

8) All neighboring subdomains are determined for each MD and TTM core. $\rightarrow$ SetNodes. $f 90$

9) A: MD cores are initialized. $\rightarrow$ SetInit.f90, RecvMD_Init.f90

B: TTM cores are initialized. $\rightarrow$ SetTTM.f90, TTMInit.f90

10) A: Each MD core performs MD integration for atoms in its subdomain, communicates with neighboring MD subdomains, and communicates with the corresponding TTM subdomains. B: Each TTM core performs TTM calculation for TTM cells in its subdomain, communicates with neighboring TTM subdomains, and communicates with the corresponding MD subdomains.

11) Collect necessary information to core (of MD_COMM_WORLD/TTM_COMM_WORLD) every time we write average quantities for the whole system (MD/TTM). $\rightarrow$ Collect. $f 90$

12) Each (MD or TTM) core writes its own restart files. $\rightarrow$ SwriteMD. $f 90 /$ Swrite1TTM.f90 


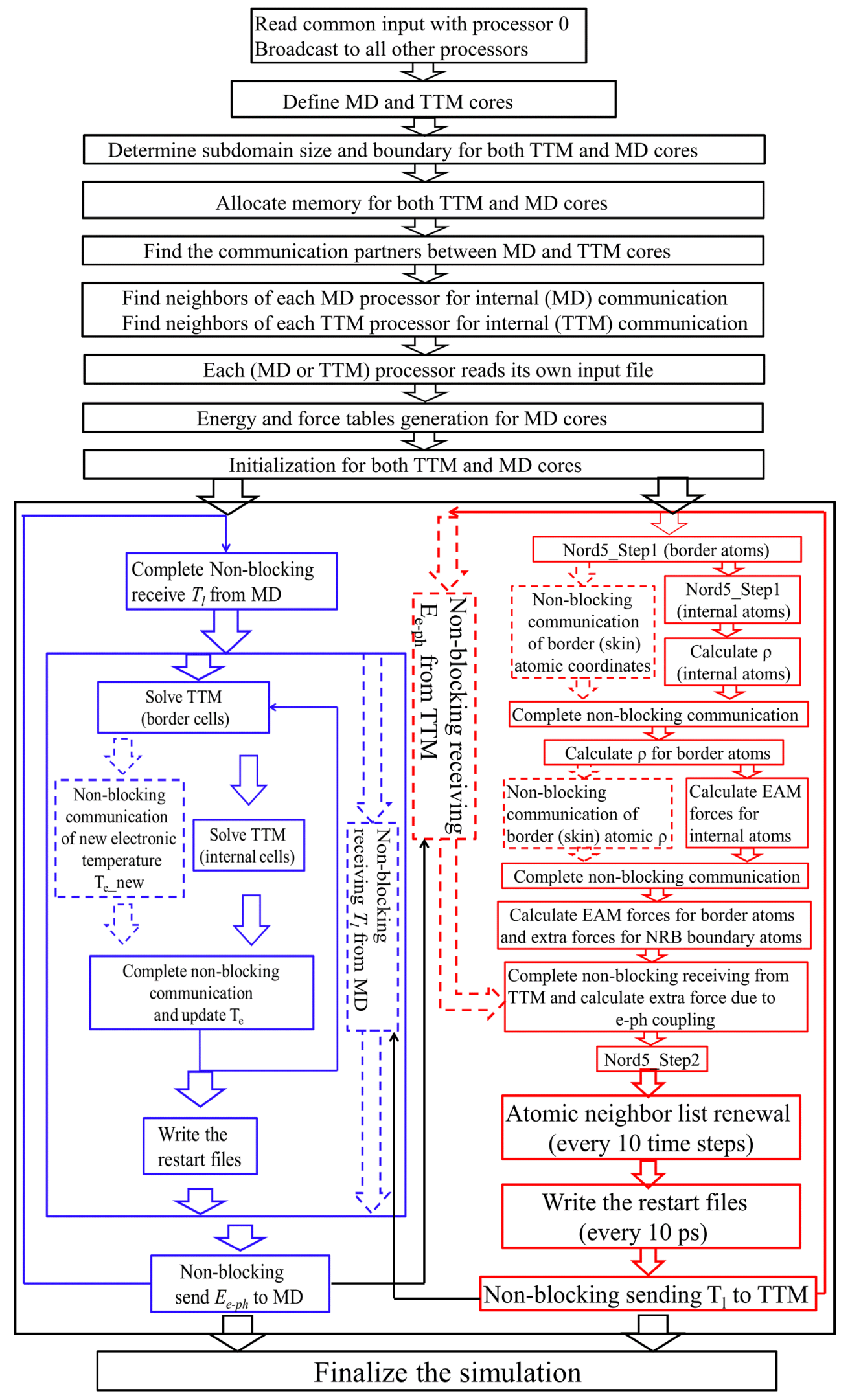

Figure 11.7: General structure for the parallel algorithm implementing the TTM-MD model. 


\subsection{Performance of MPI parallelization of the combined TTM-MD model}

The code has been used in simulations performed on up to 2100 cores at a Blue Gene/P machine (Eugene, now decommissioned) at NCCS/ORNL and demonstrated a good scalability in simulations performed for systems consisting of up to 120,000,000 atoms. Test and production runs on Kraken (NICS) have also been performed. The results of test runs performed on the Kraken cluster for a relatively small Ag system consisting of 800,000 atoms interacted with an EAM potential [59]. Perfect strong scaling behavior is observed up to $\sim 200$ cores, down to $\sim 4000$ atoms per core. First test simulations have also been performed on the Jaguar Cray XK6 System (TITAN) with the support from the Director Discretion INCITE Preparation Award (MAT043). Both weak scaling and strong scaling tests have been done Ag systems with interatomic interactions described by an EAM potential [59]. For the weak scaling test, three test runs are done. The test runs are done for 84 million atoms on 6,016 cores, 168 million atoms on 12,032 cores, and 336 million atoms on 24,064 cores. Thus, the per-core computational work is kept constant in these 3 simulations. The weak scaling behavior shown in Fig. 11.9 indicates a good weak scaling up to $\sim 24,000$ cores. For the strong scaling test, five runs with different number of cores are performed for the same Ag system consisting of 336 million atoms. As shown in Fig. 11.10, the initial tests demonstrate a good strong scaling up 25,000 cores.

On the other hand, the parallelization efficiency deviates from the ideal case as more and more computer cores are used, as shown in Figs. 11.8 and 11.10 for the strong scaling test. This can be explained by the two limits. Firstly, the system cannot be divided evenly when it is divided into smaller and smaller subdomains. To make it clear, we consider a system that consists of 50,000 atoms. When it is divided into 10 subdomains, 50,000 atoms are evenly distributed among 10 subdomains i.e., each subdomain has 5,000 atoms. When it is divided into 20 subdomains, it is very probable that atoms are not evenly distributed due to the fact that MD system is made up of discretized particles, not continuum. Imagine that one subdomain is overloaded and has 5000 atoms. This overloaded subdomain limits the parallelization scalability and the calculation is not speeded up at all even though two times more computer cores are used. Secondly, more and more communication is needed as the system is divided into smaller and smaller subdomains. Even though the calculation per computer core is perfectly reduced, the communication per core is not reduced perfectly, as more computer cores are used. In the other words, the fraction of time spent on communication is increased which limit the parallelization scalability. The extreme condition is that the core spends more time on communication than internal calculations as described by Amdahl's Law [172]. From my experience, 10,000 atoms 
per MD core is a good choice for dividing the system.

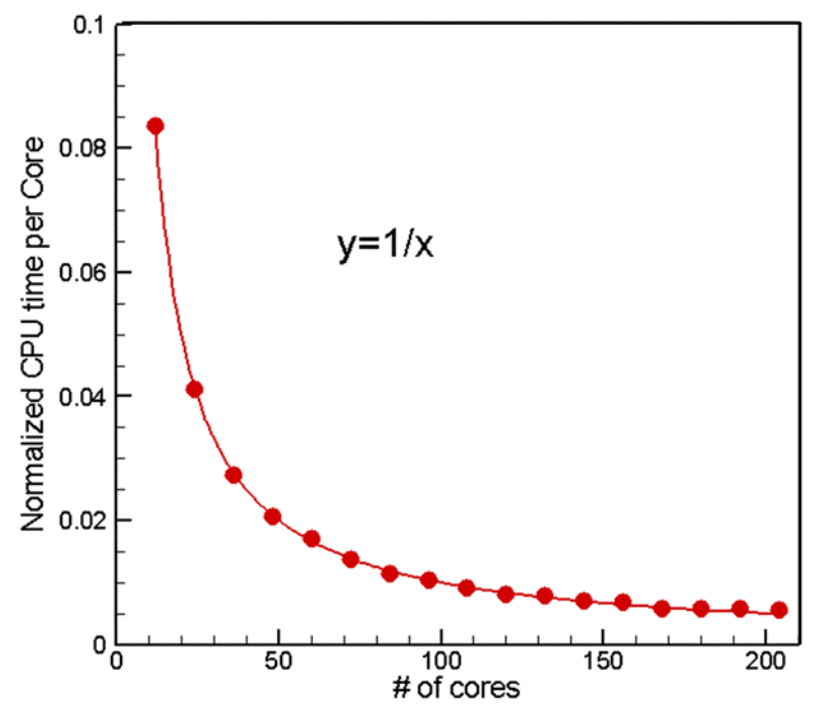

Figure 11.8: The results of test runs performed on the Kraken cluster for a system consisting of 800,000 $\mathrm{Al}$ atoms. The $\mathrm{CPU}$ time per core is normalized to 1 in the simulation performed on a single core. A perfect scalability is observed up to 204 cores, down to $\sim 4000$ atoms per core.

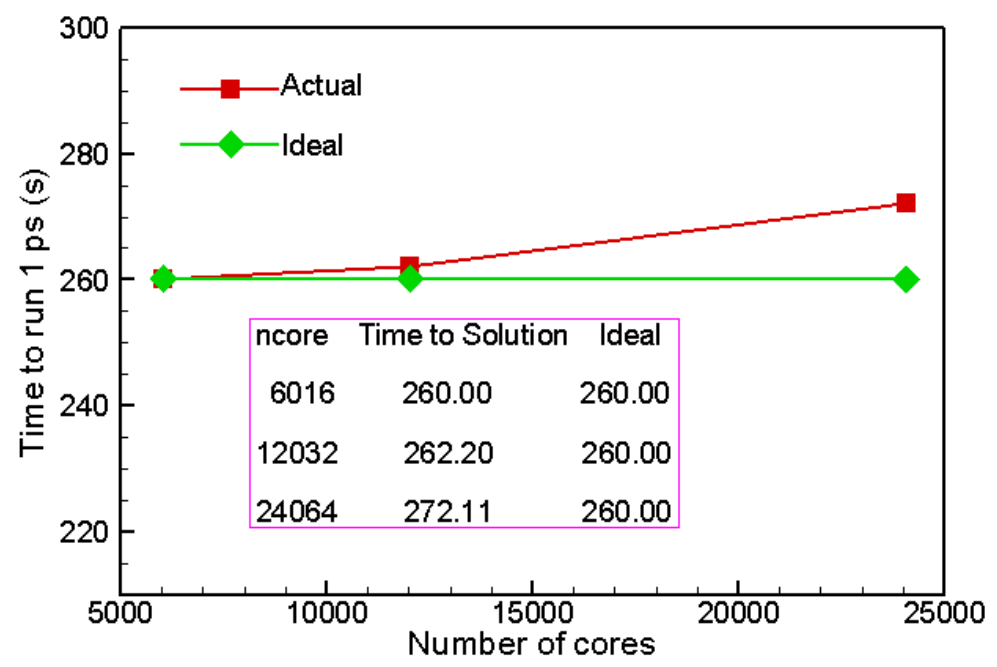

Figure 11.9: Weak scaling behavior tested in simulations of 84 million Ag atoms on 6,016 cores, 168 million Ag atoms on 12,032 cores, and 336 million Ag atoms on 24,064 cores. The MD time step is $1 \mathrm{fs}$ in the simulations. 
a)

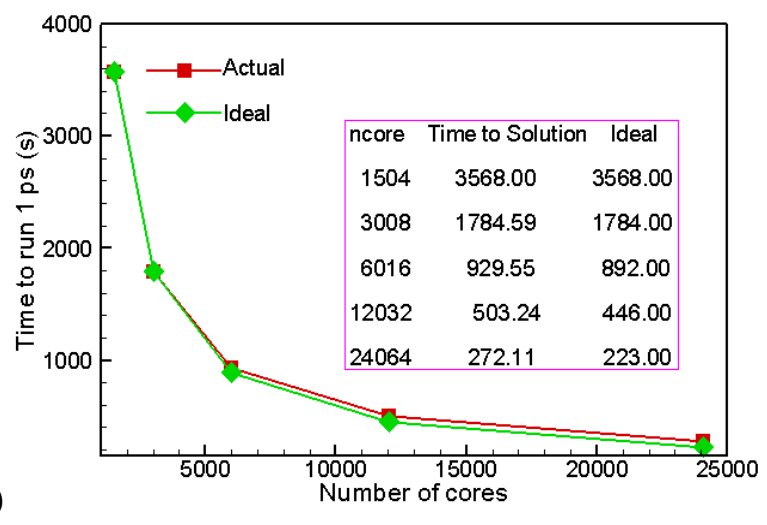

b)

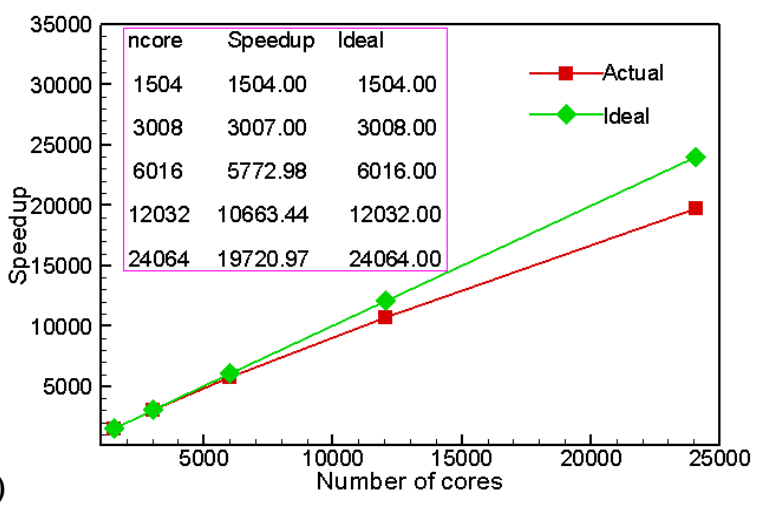

Figure 11.10: Strong scaling behavior tested in simulations of a system of 336 million $\mathrm{Ag}$ atoms performed 1504, 3008, 6016, 12032, and 24064 cores. The time to solution is shown in (a) and the corresponding strong scaling speedup is shown in (b). 


\section{Appendix B: Detailed analysis of structure of the runaway lattice-mismatched interface}

The complex structure of the three-dimensional interface as shown in Fig. 3.8 in section 3.3.4 can be thought of as formed from the two-dimensional semi-coherent interface as shown in Fig. 3.2 in section 3.2 by a sequence of dislocation reactions. It is well known that, the dislocation reactions in FCC lattice can be easily understood with the help of the Thompson tetrahedron [88] as shown in Fig. 12.1. In the following discussions, the notation of the Thompson tetrahedron is applied.

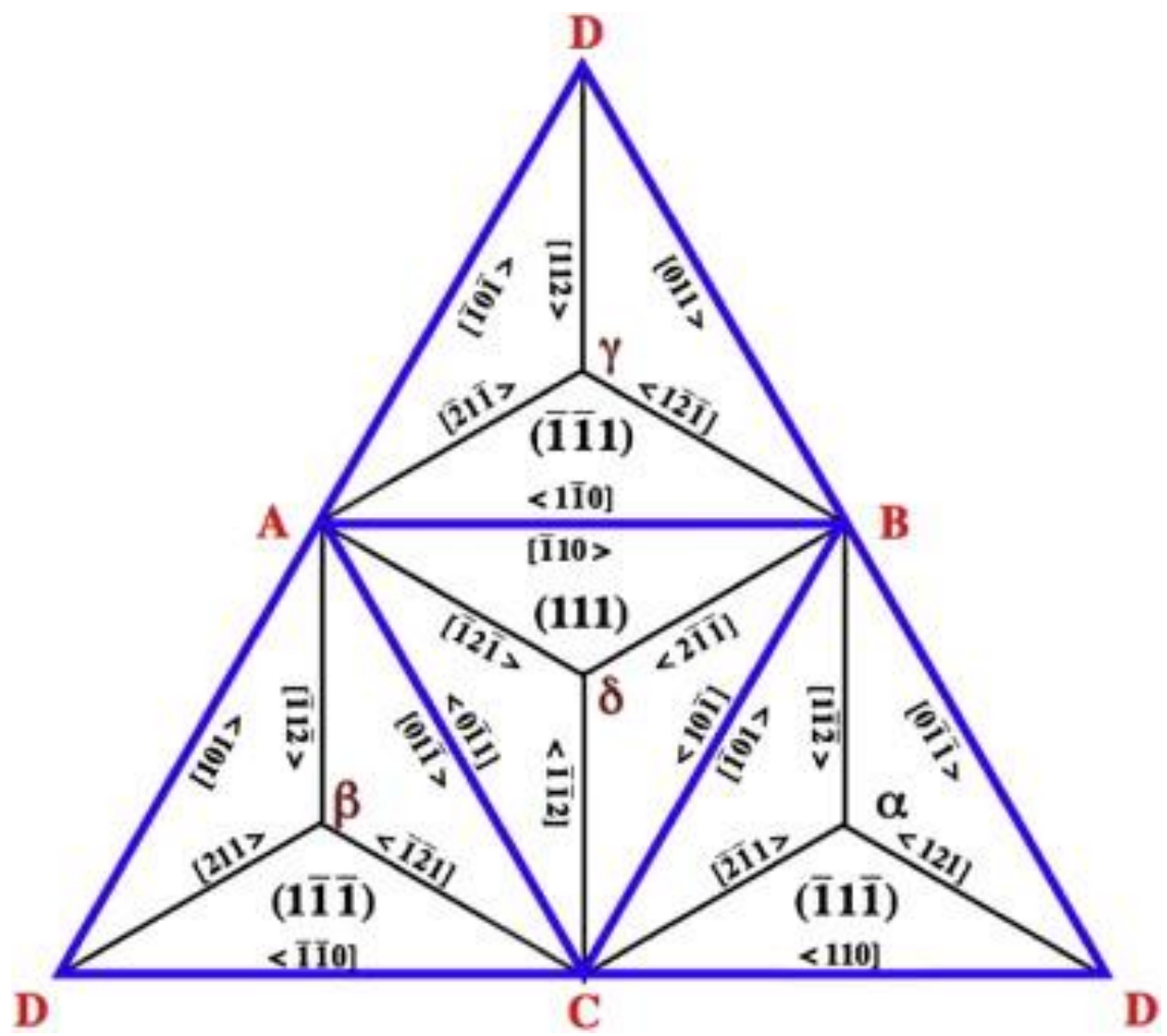

Figure 12.1: Two-dimensional representation of the Thompson tetrahedron illustrating the possible slip planes and the Burgers vectors of dislocations in an FCC crystal.

The initial square misfit dislocation network of the two-dimensional semicoherent interface is shown in Fig. 12.2a with the arrows indicating the dislocation directions. These dislocations are perfect with Burgers vector identified as $\vec{b}=\frac{1}{2}\langle 110\rangle$ ( $A B$ and $D C$ in Fig. 12.2a). 
At the first step, each of these dislocations is dissociated into two perfect dislocations as shown in Fig. 12.2b. The dislocation with Burgers vector $\mathrm{A} B=\frac{1}{2}[\overline{1} 10]$ dissociates through the reaction, $\mathrm{A} B=\frac{1}{2}[\overline{1} 10]=A C+C B=\frac{1}{2}[01 \overline{1}]+\frac{1}{2}[\overline{1} 01]$, into two perfect dislocations: one on the slip plane $A C D /(1 \overline{1} \overline{1})$ with Burgers vector $A C=\frac{1}{2}[01 \overline{1}]$, and the other on the slip plane $B C D /(\overline{1} 1 \overline{1})$ with Burgers vector $C B=\frac{1}{2}[\overline{1} 01]$. Similarly, the dislocation with Burgers vector $D C=\frac{1}{2}[110]$ dissociates $\quad$ as $\quad D C=\frac{1}{2}[110]=D B+B C=\frac{1}{2}[011]+\frac{1}{2}[10 \overline{1}]$ into two perfect dislocations, one on the slip plane $A B D /(\overline{1} \overline{1} 1)$ with Burgers vector $D B=\frac{1}{2}[011]$, and the other on the slip plane $A B C /(111)$ with Burgers vector $B C=\frac{1}{2}[10 \overline{1}]$.

At the second step, the perfect dislocations generated in the first step further dissociate into partial dislocations on the corresponding slip planes as shown in Fig. 12.2c. There are altogether 4 types of dissociations involved in this step. For example, the dislocation with Burger vector $A C=\frac{1}{2}[01 \overline{1}]$ is split into 2 partial dislocations by the reaction $A C=\frac{1}{2}[01 \overline{1}]=A \beta+\beta C=\frac{1}{6}[\overline{1} 1 \overline{2}]+\frac{1}{6}[12 \overline{1}]$ on its slip plane $A C D(1 \overline{1} \overline{1})$, one with Burgers vector $\beta C=\frac{1}{6}[12 \overline{1}]$ and the other moving away with Burgers vector $A \beta=\frac{1}{6}[\overline{1} 1 \overline{2}]$. Similarly, $C B=\frac{1}{2}[\overline{1} 01]=C \alpha+\alpha B=\frac{1}{6}[\overline{2} \overline{1} 1]+\frac{1}{6}[\overline{1} 12] \quad$ on $\quad$ the $\quad$ slip $\quad$ plane $\quad B C D /(\overline{1} 1 \overline{1})$ $D B=\frac{1}{2}[011]=D \gamma+\gamma B=\frac{1}{6}[112]+\frac{1}{6}[\overline{1} 21] \quad$ on $\quad$ the $\quad$ slip $\quad$ plane $\quad A B D /(\overline{1} \overline{1} 1) \quad, \quad$ and $B C=\frac{1}{2}[10 \overline{1}]=B \delta+\delta C=\frac{1}{6}[2 \overline{1} \overline{1}]+\frac{1}{6}[11 \overline{2}]$ on the slip plane $A B C /(111)$.

Finally, the partial dislocations dissociated in the second step react to generate the stairrod dislocations at the intersections of the $\{111\}$ planes as seen in Fig. 12.2d. There are altogether 6 types of reactions involved in this step as indicated in Fig. 12.2d. For the first type, 
the partial dislocation $\beta C=\frac{1}{6}[12 \overline{1}]$ reacts with $C \alpha=\frac{1}{6}[\overline{2} \overline{1} 1]$ to generate a new dislocation $\beta \alpha=\frac{1}{6}[\overline{1} 10]$ by $\beta C+C \alpha=\frac{1}{6}[12 \overline{1}]+\frac{1}{6}[\overline{2} \overline{1} 1]=\beta \alpha=\frac{1}{6}[\overline{1} 10]$ at the intersection [110] of plane $A C D /(1 \overline{1} \overline{1})$ and plane $B C D /(\overline{1} 1 \overline{1})$. For the second type, the partial dislocation $\gamma B=\frac{1}{6}[\overline{1} 21]$ reacts with $B \delta=\frac{1}{6}[2 \overline{1} \overline{1}] \quad$ to generate a new dislocation $\quad \gamma \delta=\frac{1}{6}[110]$ by $\gamma B+B \delta=\frac{1}{6}[\overline{1} 21]+\frac{1}{6}[2 \overline{1} \overline{1}]=\frac{1}{6}[110]=\gamma \delta$ at the intersection $[1 \overline{1} 0]$ of plane $A B D /(\overline{1} \overline{1} 1)$ and plane $A B C /(111)$. The dislocations generated in these 2 types of reactions are well known as the "Lomer-Cottrell locks". For the third type, $\alpha B=\frac{1}{6}[\overline{1} 12]$ reacts with $D \gamma=\frac{1}{6}[112]$ to generate a new dislocation $\gamma \alpha / D B=\frac{1}{3}[100]$ by $B \alpha+D \gamma=-\frac{1}{6}[\overline{1} 12]+\frac{1}{6}[112]=\frac{1}{3}[100]=\gamma \alpha / D B$ at the intersection $[0 \overline{1} \overline{1}]$ of plane $B C D /(\overline{1} 1 \overline{1})$ and plane $A B D /(\overline{1} \overline{1} 1)$ ( $B \alpha$ is used in the reaction instead of $\alpha B$ to account for the opposite dislocation direction). For the fourth type, $\alpha B=\frac{1}{6}[\overline{1} 12]$ reacts with $\delta C=\frac{1}{6}[11 \overline{2}]$ to generate a new dislocation $\alpha \delta / B C=\frac{1}{3}[010]$ by $\alpha B+\delta C=\frac{1}{6}[\overline{1} 12]+\frac{1}{6}[11 \overline{2}]=\frac{1}{3}[010]=\alpha \delta / B C$ at the intersection $[10 \overline{1}]$ of plane $B C D /(\overline{1} 1 \overline{1})$ and plane $A B C /(111)$. For the fifth type, $\delta C=\frac{1}{6}[11 \overline{2}]$ reacts with $A \beta=\frac{1}{6}[\overline{1} 1 \overline{2}]$ to generate a new dislocation $\beta \delta / A C=\frac{1}{3}[\overline{1} 00]$ by $C \delta+A \beta=-\frac{1}{6}[11 \overline{2}]+\frac{1}{6}[\overline{1} 1 \overline{2}]=\frac{1}{3}[\overline{1} 00]=\beta \delta / A C$ at the intersection $[01 \overline{1}]$ of plane $A B C /(111)$ and plane $A C D /(1 \overline{1} \overline{1})$. For the last type, $A \beta=\frac{1}{6}[\overline{1} 1 \overline{2}]$ reacts with $D \gamma=\frac{1}{6}[112]$ by $\beta A+\gamma D=-\frac{1}{6}[\overline{1} 1 \overline{2}]-\frac{1}{6}[112]=\frac{1}{3}[0 \overline{1} 0]=\gamma \beta / D A$ at the intersection $[\overline{1} 0 \overline{1}]$ of plane $A C D(1 \overline{1} \overline{1})$ and plane $A B D(\overline{1} \overline{1} 1)$. The dislocations generated in these 4 types of reactions are well known as the "Hirth locks". The final dislocation network is shown in Fig. 12.2e. 
As we know, the Burgers vector is conserved in the dislocation network, i.e., in each dislocation node, the sum of Burgers vectors of the entering dislocations should be equal to the sum of the Burgers vectors of the leaving dislocations. We can apply this criterion to check the correctness of our above analysis. For the dislocation node marked by black circle, all dislocations are entering. The sum of Burgers vectors is $\gamma \alpha / D B+\alpha \delta B C+\beta \delta / A C+\gamma \beta / D A=\frac{1}{3}[100]+\frac{1}{3}[010]+\frac{1}{3}[\overline{1} 00]+\frac{1}{3}[0 \overline{1} 0]=[000]$ as expected. For the dislocation node marked by purple circle, the sum of Burgers vectors of entering dislocations is $\beta \alpha+\gamma \delta=\frac{1}{6}[\overline{1} 10]+\frac{1}{6}[110]=\frac{1}{3}[010]$ and is indeed equal to the sum of Burgers vectors of leaving dislocations, $\gamma \alpha / D B+\alpha \delta B C+\beta \delta / A C+\gamma \beta / D A+\beta \alpha+\gamma \delta=[000]+\beta \alpha+\gamma \delta=\beta \alpha+\gamma \delta=\frac{1}{3}[010]$. So the Burgers vector is conserved.

In summary, by a sequence of dislocation reactions, a three-dimensional interface structure consisting of a periodic array of stacking fault pyramids outlined by stair-rod partial dislocations is generated. The partial dislocations in the base of each pyramid are identified as Lomer-Cottrell locks with Burgers vector $\vec{b}=\frac{1}{6}\langle 110\rangle$ and the partial dislocations forming the edges of the pyramids are Hirth locks with Burgers vector $\vec{b}=\frac{1}{3}\langle 010\rangle$. The fact that these stairrod dislocations at the pyramid edges are immobile and the faces of the pyramids are intrinsic stacking faults presents a strong barrier to dislocation slip and results in the effective hardening of the interfacial region. 
(a)

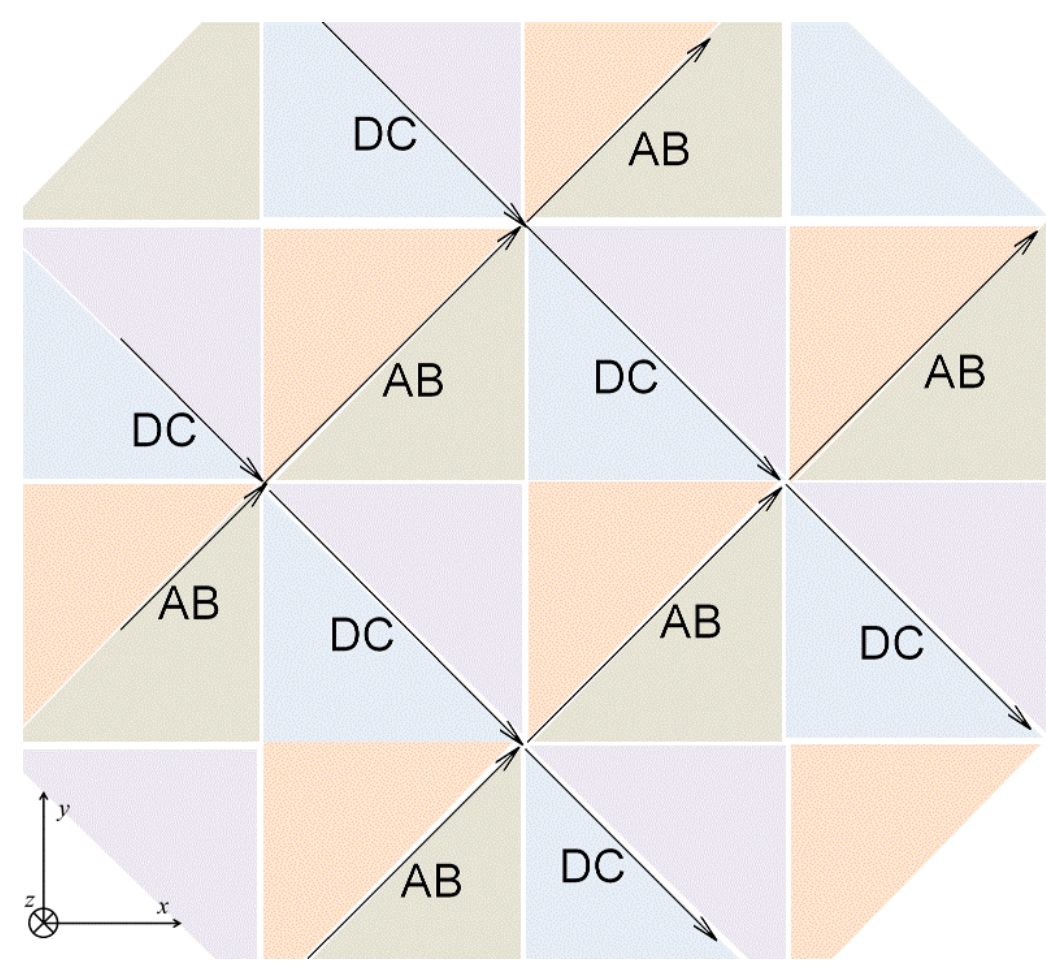

(b)

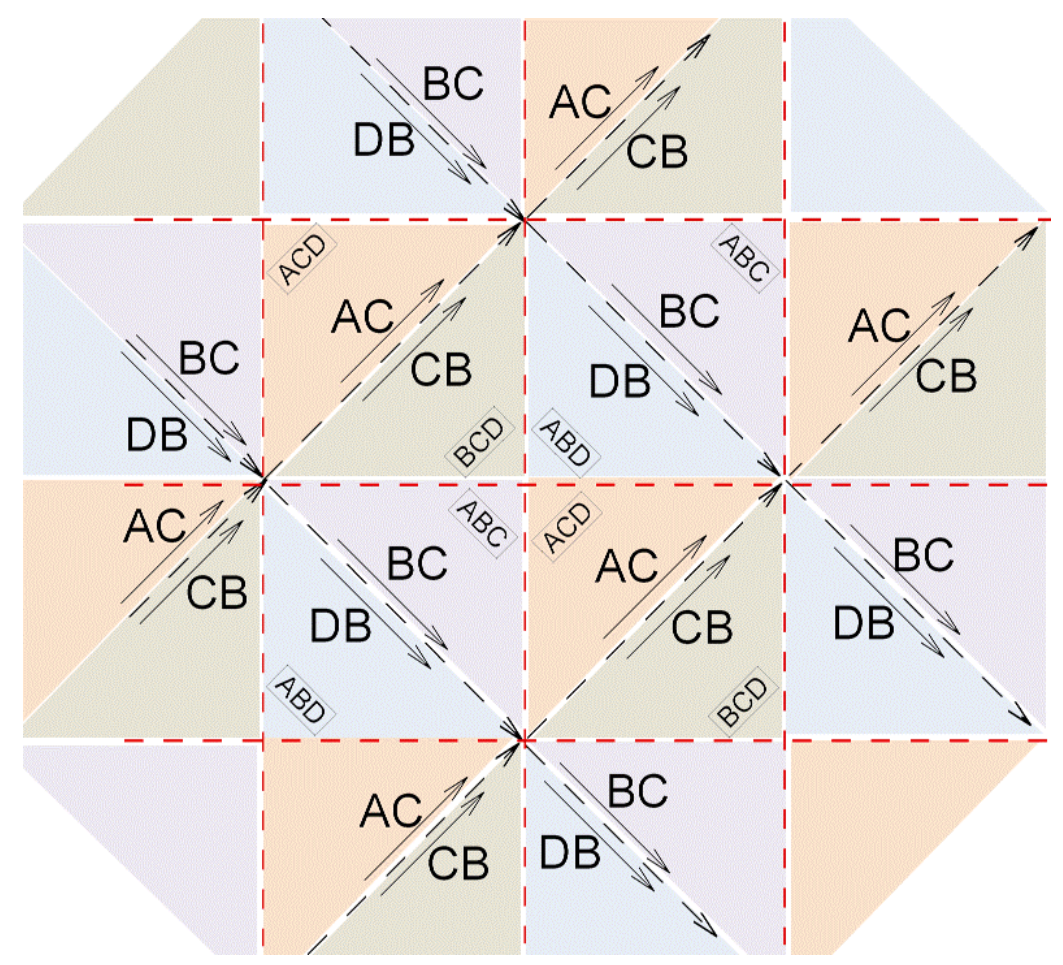


(c)

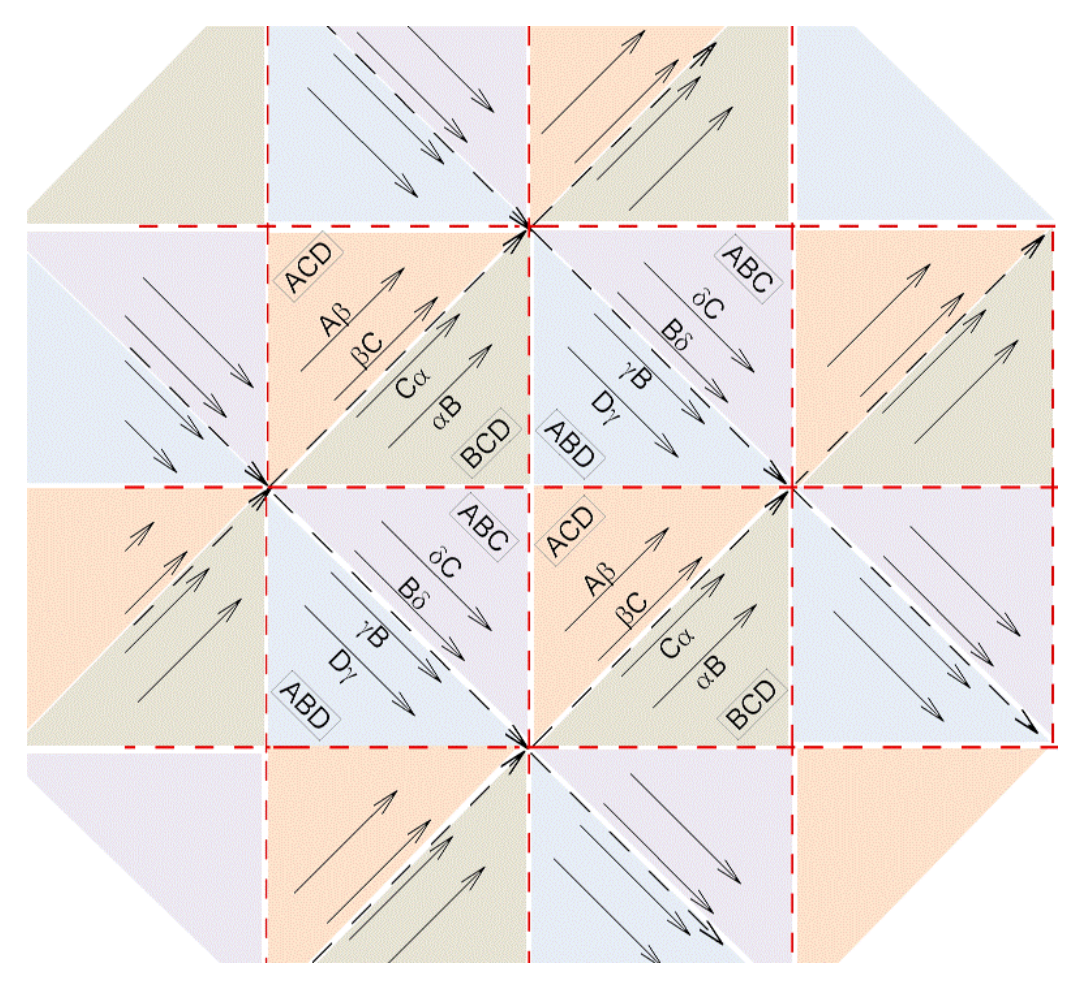

(d)

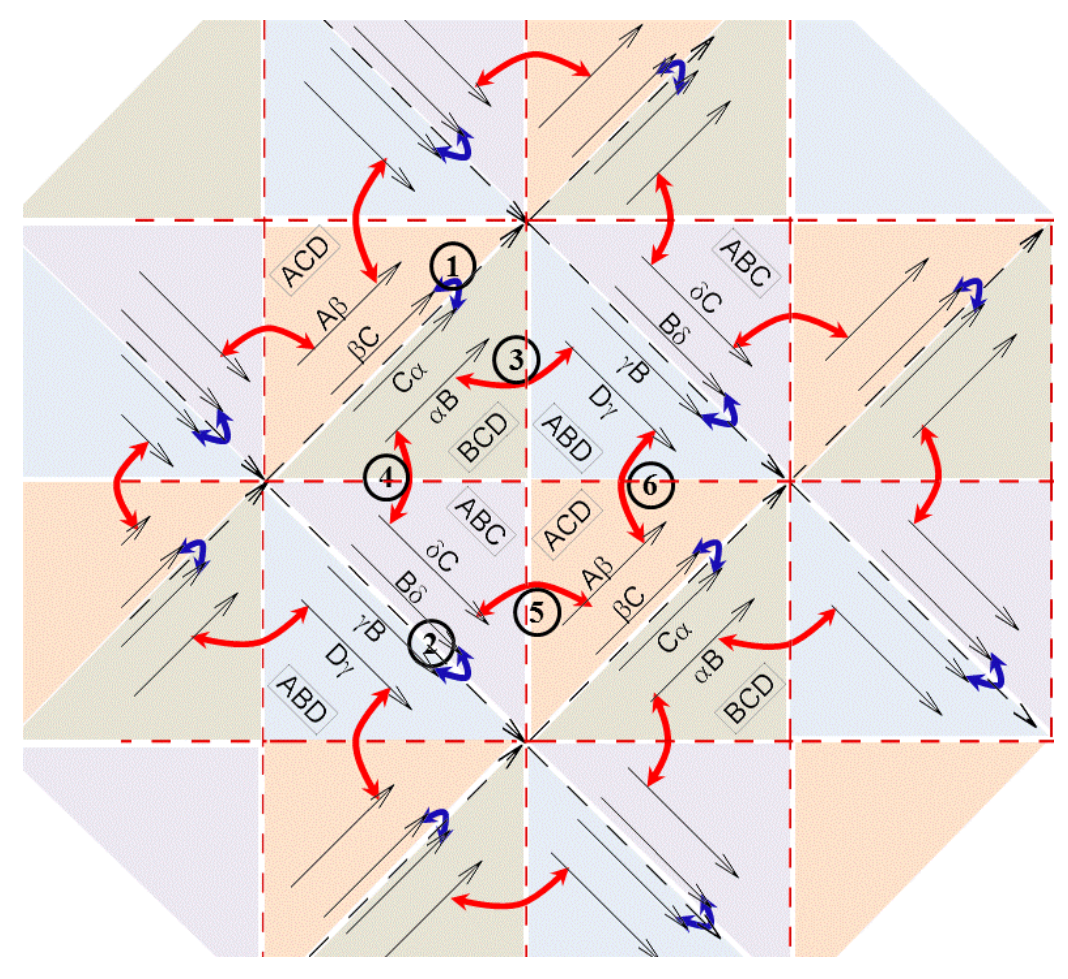


(e)

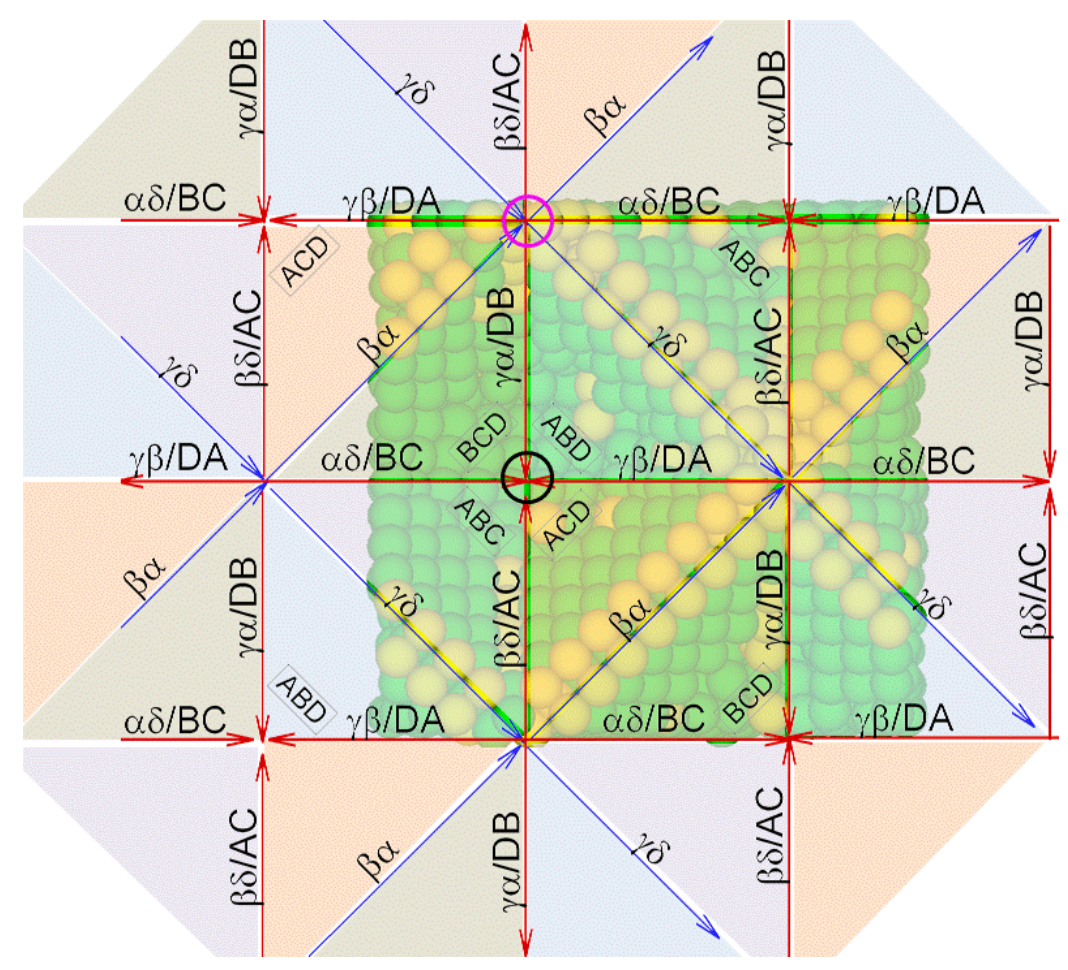

Figure 12.2: Dislocation reactions involved in the formation of the three-dimensional latticemismatched interface. (a) Square dislocation networks of the two-dimension semi-coherent interface. (b) The first step: dissociation into two perfect dislocations. (c) The second step dissociation into two partial dislocations. (d) The reaction of the partial dislocations to form the three-dimensional dislocation network of the new lattice-mismatched interface shown in (e). 


\section{Appendix C: Analytical derivation on nano-structuring equations}

\subsection{Diffusion barriers and jump probabilities}

As discussed in section 9.3, the acoustic strain field induces the diffusion barrier inhomogeneity and diffusion barrier left-right asymmetry. This can be understood by the linear strain dependence of binding/saddle-point energy, $E_{B, S}=E_{B, S}{ }^{0}+\beta_{B, S} e$, and the strain gradient of acoustic strain field. For the diffusion barriers to the left $E_{d, p}(x)$ and to the right $E_{d, q}(x)$ as shown in Fig. 13.1, they can be calculated as,

$$
\begin{aligned}
& E_{d, p}(x)=E_{S}^{p}-E_{B}(x)=E_{S}\left(x-\frac{b}{2}\right)-E_{B}(x)=E_{S}^{0}+\beta_{S} e\left(x-\frac{b}{2}\right)-\left[E_{B}^{0}+\beta_{B} e(x)\right] \\
& \approx\left(E_{S}^{0}-E_{B}^{0}\right)+\beta_{S}\left(e(x)-\frac{b}{2} \frac{\partial e}{\partial x}\right)-\beta_{B} e(x) \\
& =E_{d}^{0}+\left(\beta_{S}-\beta_{B}\right) e(x)-\frac{b}{2} \beta_{S} \frac{\partial e}{\partial x} \\
& =E_{d}^{0}+\gamma e(x)-\frac{b}{2} \delta e_{x} \\
& =E_{d}^{0}+\Delta E_{d, p} \\
& E_{d, q}(x)=E_{S}^{q}-E_{B}(x)=E_{S}\left(x+\frac{b}{2}\right)-E_{B}(x)=E_{S}^{0}+\beta_{S} e\left(x+\frac{b}{2}\right)-\left[E_{B}^{0}+\beta_{B} e(x)\right] \\
& \approx\left(E_{S}^{0}-E_{B}^{0}\right)+\beta_{S}\left(e(x)+\frac{b}{2} \frac{\partial e}{\partial x}\right)-\beta_{B} e(x) \\
& =E_{d}^{0}+\left(\beta_{S}-\beta_{B}\right) e(x)+\frac{b}{2} \beta_{S} \frac{\partial e}{\partial x} \\
& =E_{d}^{0}+\gamma e(x)+\frac{b}{2} \delta e_{x}^{\prime} \\
& =E_{d}^{0}+\Delta E_{d, q}
\end{aligned}
$$

where $b$ is the distance between neighboring equilibrium sites, $e_{x}^{\prime}=\frac{\partial e}{\partial x}$ is the local strain gradient, $E_{S}^{0}, E_{B}^{0}$ and $E_{d}^{0}$ are unperturbed saddle-point energy, binding energy and diffusion barrier, $E_{S}^{p}$ and $E_{S}^{q}$ are the saddle-point energies on the left side and on the 
right, $\Delta E_{d, p} \approx \gamma e(x)-\frac{b}{2} \delta e_{x}^{\prime}$ and $\Delta E_{d, q} \approx \gamma e(x)+\frac{b}{2} \delta e_{x}^{\prime}$ are perturbations of diffusion barriers to the left and right, $\beta_{S}$ and $\beta_{B}$ are the coefficients describing the linear strain dependence of saddle-point energy and binding energy, and $\gamma$ and $\delta$ are the coefficients describing the diffusion barrier inhomogeneity, $E_{d}(e)=\frac{E_{d, p}+E_{d, q}}{2} \approx E_{d}^{0}+\gamma e, \quad$ and diffusion barrier asymmetry, $E_{d, q}(x)-E_{d, p}(x) \approx \delta b e_{x}^{\prime}$.

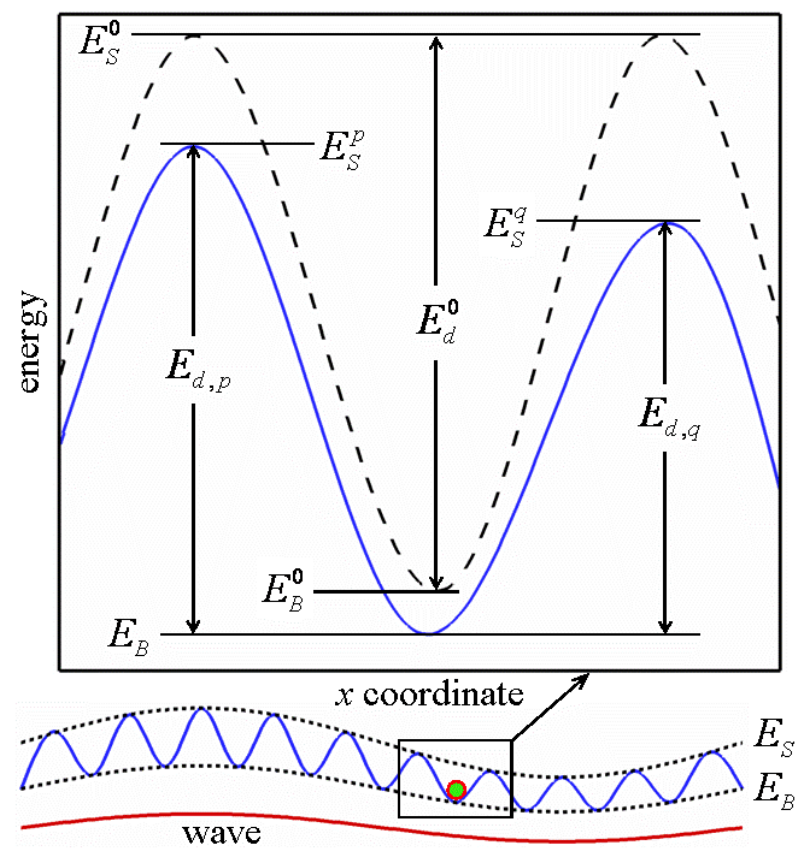

Figure 13.1: Schematic illustration of the effect of surface strain generated by a SAW on the energy landscape for adatom diffusion. The dashed and solid lines show the energy barriers for adatom diffusion without and with the SAW, respectively. This figure is same as Fig. 9.1.

The difference of the left, $E_{d, p}(x)$, and right, $E_{d, q}(x)$, diffusion barriers leads to the difference of probabilities for the adatom jump to the left $r_{p}$ and right $r_{q}$ :

$$
\begin{aligned}
& r_{p}(x, t)=R_{0} \exp \left(-\frac{E_{d, p}(x, t)}{k_{B} T_{0}}\right)=R_{0} \exp \left(-\frac{E_{d}{ }^{0}+\Delta E_{d, p}}{k_{B} T_{0}}\right) \approx r_{0}\left[1-\frac{\Delta E_{d, p}}{k_{B} T_{0}}+\frac{1}{2}\left(\frac{\Delta E_{d, p}}{k_{B} T_{0}}\right)^{2}+\ldots\right) \\
& r_{q}(x, t)=R_{0} \exp \left(-\frac{E_{d, q}(x, t)}{k_{B} T_{0}}\right)=R_{0} \exp \left(-\frac{E_{d}{ }^{0}+\Delta E_{d, q}}{k_{B} T_{0}}\right) \approx r_{0}\left[1-\frac{\Delta E_{d, q}}{k_{B} T_{0}}+\frac{1}{2}\left(\frac{\Delta E_{d, q}}{k_{B} T_{0}}\right)^{2}+\ldots\right)
\end{aligned}
$$


where $R_{0}$ is the so-called attempt frequency related to the vibrational frequency of a surface adatom, and $r_{0}$ is the unperturbed jump probability. We retain only the first two terms in the power-law expansion of the exponential Arrhenius factors determining the probabilities of adatom jump between the neighboring atomic cells. This approximation is accurate when the diffusion barrier perturbation is smaller than $k_{B} T_{0}$. For the convenience, two variables $R$ and $Q$ are introduced below, with $R=\frac{r_{p}+r_{q}}{2}-r_{0}$ describing the mean probability variation and $Q=r_{p}-r_{q}$ describing the left-right asymmetry.

$R=\frac{r_{p}+r_{q}}{2}-r_{0} \approx-\frac{r_{0}\left(\Delta E_{d, p}+\Delta E_{d, q}\right)}{2 k_{B} T_{0}}+\frac{r_{0}\left(\Delta E_{d, p}{ }^{2}+\Delta E_{d, q}{ }^{2}\right)}{4\left(k_{B} T_{0}\right)^{2}}$

$Q=r_{p}-r_{q} \approx \frac{r_{0}\left(\Delta E_{d, q}-\Delta E_{d, p}\right)}{k_{B} T_{0}}+\frac{r_{0}\left(\Delta E_{d, p}{ }^{2}-\Delta E_{d, p}{ }^{2}\right)}{2\left(k_{B} T_{0}\right)^{2}}$

By plugging in $\Delta E_{d, p} \approx \gamma e(x)-\frac{b}{2} \delta e_{x}^{\prime}$ and $\Delta E_{d, q} \approx \gamma e(x)+\frac{b}{2} \delta e_{x}^{\prime}$ into Eq. (5) and (6) and keeping the terms up to the first order of $k b(k b<<1$ in the long acoustic wave assumption) and second order of strain $e, R$ and $Q$ can be expressed as below.

$$
\begin{aligned}
& R=\frac{r_{p}+r_{q}}{2}-r_{0} \approx-\frac{r_{0} \gamma e}{k_{B} T_{0}}+\frac{r_{0}}{2}\left(\frac{\gamma e}{k_{B} T_{0}}\right)^{2}=R^{(1)}+R^{(2)} \\
& \text { with } R^{(1)}=-\frac{r_{0} \gamma e}{k_{B} T_{0}}, \quad R^{(2)}=\frac{r_{0}}{2}\left(\frac{\gamma e}{k_{B} T_{0}}\right)^{2} \\
& Q=r_{p}-r_{q} \approx \frac{r_{0} b \delta \partial e / \partial x}{k_{B} T_{0}}-\frac{r_{0} b \gamma \delta e \partial e / \partial x}{\left(k_{B} T_{0}\right)^{2}}=Q^{(1)}+Q^{(2)} \\
& \text { with } Q^{(1)}=\frac{r_{0} b \delta \partial e / \partial x}{k_{B} T_{0}}, \quad Q^{(2)}=-\frac{r_{0} b \gamma \delta e \partial e / \partial x}{\left(k_{B} T_{0}\right)^{2}}
\end{aligned}
$$

\subsection{D kinetic equation for adatom's density}

The time-averaged structuring of adatoms in the presence of a standing SAW can be considered by solving 1D kinetic equation 


$$
\begin{aligned}
\partial n(x, t) / \partial t=v_{+}(x, t)-v_{-}(x, t)= & {\left[n(x+b, t) r_{p}(x+b, t)+n(x-b, t) r_{q}(x-b, t)\right], } \\
& -\left[n(x, t) r_{p}(x, t)+n(x, t) r_{q}(x, t)\right]
\end{aligned},
$$

where $n(x, t)$ is the adatom density at a site located at $x$ at time $t$, $v_{+}(x, t)=n(x+b, t) r_{p}(x+b, t)+n(x-b, t) r_{q}(x-b, t)$ and $v_{-}(x, t)=n(x, t) r_{p}(x, t)+n(x, t) r_{q}(x, t)$ are the rates of adatom jumps from/to neighboring sites with coordinates $x \pm b . \quad r_{p}$ and $r_{q}$ can be expressed by $R$ and $Q$ from Eq. (5) and (6), i.e., $r_{p}=R+r_{0}+\frac{Q}{2}$ and $r_{q}=R+r_{0}-\frac{Q}{2}$. Then we can rewrite Eq. (9), retaining the terms up to second order of $k b$.

$$
\begin{aligned}
\frac{\partial n}{\partial t}= & v_{+}(x)-v_{-}(x) \\
= & {\left[n(x+b) r_{p}(x+b)+n(x-b) r_{q}(x-b)\right] } \\
& -\left[n(x) r_{p}(x)+n(x) r_{q}(x)\right] \\
= & {\left[n(x+b)\left(R(x+b)+r_{0}+\frac{Q(x+b)}{2}\right)+n(x-b)\left(R(x-b)+r_{0}-\frac{Q(x-b)}{2}\right)\right] } \\
& -\left[n(x)\left(R(x)+r_{0}+\frac{Q(x)}{2}\right)+n(x)\left(R(x)+r_{0}-\frac{Q(x)}{2}\right)\right] \\
= & {[n(x+b) R(x+b)-n(x) R(x)]+[n(x-b) R(x-b)-n(x) R(x)] } \\
& +\frac{1}{2}[n(x+b) Q(x+b)-n(x) Q(x)]-\frac{1}{2}[n(x-b) Q(x-b)-n(x) Q(x)] \\
& +r_{0}[n(x+b)-n(x)]+r_{0}[n(x-b)-n(x)] \\
\approx & {\left[\frac{\partial(n R)}{\partial x} b+\frac{b^{2}}{2} \frac{\partial^{2}(n R)}{\partial x^{2}}\right]+\left[\frac{\partial(n R)}{\partial x}(-b)+\frac{(-b)^{2}}{2} \frac{\partial^{2}(n R)}{\partial x^{2}}\right] } \\
& +\frac{1}{2}\left[\frac{\partial(n Q)}{\partial x} b+\frac{b^{2}}{2} \frac{\partial^{2}(n Q)}{\partial x^{2}}\right]-\frac{1}{2}\left[\frac{\partial(n Q)}{\partial x}(-b)+\frac{(-b)^{2}}{2} \frac{\partial^{2}(n Q)}{\partial x^{2}}\right] \\
& +r_{0}\left[\frac{\partial n}{\partial x} b+\frac{b^{2}}{2} \frac{\partial^{2} n}{\partial x^{2}}\right]+r_{0}\left[\frac{\partial n}{\partial x}(-b)+\frac{(-b)^{2}}{2} \frac{\partial^{2} n}{\partial x^{2}}\right] \\
= & b^{2} \frac{\partial^{2}(n R)}{\partial x^{2}}+b \frac{\partial(n Q)}{\partial x}+r_{0} b^{2} \frac{\partial^{2} n}{\partial x^{2}}
\end{aligned}
$$


We denote the uniform unperturbed density as $n_{0}$ and its dimensionless perturbation as $N=\left(n-n_{0}\right) / n_{0}$. Eq. (10) can be further rewritten in $N$ by substituting $n$ with $n=n_{0}+N n_{0}$,

$$
\begin{aligned}
\frac{\partial N(x, t)}{\partial t}-r_{0} b^{2} \frac{\partial^{2} N(x, t)}{\partial x^{2}} & \approx b^{2} \frac{\partial^{2}}{\partial x^{2}}[R(x, t)+R(x, t) N(x, t)] \\
& +b \frac{\partial}{\partial x}[Q(x, t)+Q(x, t) N(x, t)]
\end{aligned}
$$

As we see Eq. (7) and (8), $R$ and $Q$ contain both linear-in-strain, $R^{(1)}$ and $Q^{(1)}$, and quadratic-in-strain components, $R^{(2)}$ and $Q^{(2)}$. The quadratic-in-strain component can give direct time-averaged effect on the adatom density perturbation $N(x, t)$ of second order on strain, so called "direct mechanism". For the linear-in-strain component, it results in an instantaneous adatom density perturbation $N^{(1)}(x, t)$ of first order on strain, which gives zero time-averaged effect on the adatom density perturbation, i.e. $\left\langle N^{(1)}(x, t)\right\rangle=0$. However, $N^{(1)}$ can combine with $R^{(1)}$ and $Q^{(1)}$, through the terms of $R N$ and $Q N$, to produce non-zero time-averaged effect, so called "cascade mechanism". In the following, the direct mechanism and cascade mechanism are discussed separately.

\subsection{Direct mechanism}

Plug in the quadratic-in-strain components, $R^{(2)}$ and $Q^{(2)}$, into Eq. (11), keep only the terms up to second order on strain $e$, and apply time-averaging over acoustic wave period, we have,

$$
\begin{aligned}
\frac{\partial<N>}{\partial t}-p_{0} b^{2} \frac{\partial^{2}<N>}{\partial x^{2}} \approx<b^{2} \frac{\partial^{2}}{\partial x^{2}}\left[R^{(2)}(x, t)\right]+b \frac{\partial}{\partial x}\left[Q^{(2)}(x, t)\right]> \\
=<b^{2} \frac{\partial^{2}}{\partial x^{2}}\left[\frac{r_{0} \gamma^{2} e^{2}}{2\left(k_{B} T_{0}\right)^{2}}\right]+b \frac{\partial}{\partial x}\left[\frac{-r_{0} b \gamma \delta e^{\prime}{ }_{x} e}{\left(k_{B} T_{0}\right)^{2}}\right]> \\
=<b^{2} \frac{\partial^{2}}{\partial x^{2}}\left[\frac{r_{0} \gamma^{2} e^{2}}{2\left(k_{B} T_{0}\right)^{2}}\right]+b \frac{\partial}{\partial x}\left[\frac{-r_{0} b \gamma \delta}{\left(k_{B} T_{0}\right)^{2}} \frac{1}{2} \frac{\partial e^{2}}{\partial x}\right]> \\
=<\frac{r_{0} \gamma^{2} b^{2}}{2\left(k_{B} T_{0}\right)^{2}} \frac{\partial^{2} e^{2}}{\partial x^{2}}-\frac{r_{0} \gamma \delta b^{2}}{2\left(k_{B} T_{0}\right)^{2}} \frac{\partial^{2} e^{2}}{\partial x^{2}}> \\
=b^{2} r_{0} \frac{\gamma(\gamma-\delta)}{2\left(k_{B} T_{0}\right)^{2}} \frac{\partial^{2}<e^{2}>}{\partial x^{2}}
\end{aligned}
$$


The solution can be easily found for Eq. (12):

$$
\begin{aligned}
\bar{N}(x, t) & \approx\left[1-\exp \left(-4 k^{2} b^{2} r_{0} t\right)\right] \frac{\gamma(\gamma-\delta)}{8\left(k_{B} T_{0}\right)^{2}} e_{0}^{2} \cos (2 k x) & \\
& =[1-\exp (-t / \tau)] \frac{\gamma(\gamma-\delta)}{8\left(k_{B} T_{0}\right)^{2}} e_{0}^{2} \cos (2 k x) & \text { with } \tau=\left(4 k^{2} b^{2} r_{0}\right)^{-1}
\end{aligned}
$$

\subsection{Cascade mechanism}

Plug in the linear-in-strain components, $R^{(1)}$ and $Q^{(1)}$, into Eq. (11) and keep only the terms up to first order on strain $e$, we have,

$$
\begin{aligned}
\frac{\partial N^{(1)}}{\partial t}-r_{0} b^{2} \frac{\partial^{2} N^{(1)}}{\partial x^{2}} & \approx b^{2} \frac{\partial^{2}}{\partial x^{2}}\left[R^{(1)}(x, t)\right]+b \frac{\partial}{\partial x}\left[Q^{(1)}(x, t)\right] \\
& =b^{2} \frac{\partial^{2}}{\partial x^{2}}\left[\frac{-r_{0} \gamma e}{k_{B} T_{0}}\right]+b \frac{\partial}{\partial x}\left[\frac{r_{0} b \delta e_{x}}{k_{B} T_{0}}\right] \\
& =b^{2} \frac{-r_{0} \gamma}{k_{B} T_{0}} \frac{\partial^{2} e}{\partial x^{2}}+\frac{r_{0} b^{2} \delta}{k_{B} T_{0}} \frac{\partial^{2} e}{\partial x^{2}} \\
& =\frac{r_{0} b^{2}}{k_{B} T_{0}}(\delta-\gamma) \frac{\partial^{2} e}{\partial x^{2}}
\end{aligned}
$$

The solution to Eq. (14) can be easily found:

$$
\begin{aligned}
N^{(1)} & =\operatorname{Re}\left\{\frac{-r_{0} b^{2}(\delta-\gamma) k^{2} e_{0}}{\left(i \Omega+r_{0} b^{2} k^{2}\right) k_{B} T_{0}} \sin (k x) \exp (i \Omega t)\right\} \\
& =\frac{(\gamma-\delta) e_{0}}{k_{B} T_{0}}\left[\frac{\left(k^{2} b^{2} r_{0}\right)^{2}}{\left(k^{2} b^{2} r_{0}\right)^{2}+\Omega^{2}} \sin (k x) \cos (\Omega t)+\frac{\left(k^{2} b^{2} r_{0}\right) \Omega}{\left(k^{2} b^{2} r_{0}\right)^{2}+\Omega^{2}} \sin (k x) \sin (\Omega t)\right] \\
& =U \sin (k x) \cos (\Omega t)+W \sin (k x) \sin (\Omega t)
\end{aligned}
$$

with $U=\frac{(\gamma-\delta) e_{0}}{k_{B} T_{0}} \frac{\left(k^{2} b^{2} r_{0}\right)^{2}}{\left(k^{2} b^{2} r_{0}\right)^{2}+\Omega^{2}}$ and $W=\frac{(\gamma-\delta) e_{0}}{k_{B} T_{0}} \frac{\left(k^{2} b^{2} r_{0}\right) \Omega}{\left(k^{2} b^{2} r_{0}\right)^{2}+\Omega^{2}}$.

As clearly seen from Eq. (15), $N^{(1)}$ gives zero time-averaged effect, $\left\langle N^{(1)}(x, t)\right\rangle=0$. But $N^{(1)}$ has the component $\propto \cos (\Omega t)$, which can combine with $R^{(1)} \propto \cos (\Omega t)$ and $Q^{(1)} \propto \cos (\Omega t)$, through the terms of $R N$ and $Q N$, to produce non-zero time-averaged effect. Insert $N^{(1)}, R^{(1)}$, and $Q^{(1)}$ into the right side of Eq. (11), retain the terms up to $2^{\text {nd }}$ order of strain, and apply time-averaging over acoustic wave period, we have 


$$
\begin{aligned}
\frac{\partial \bar{N}^{(2)}}{\partial t}-r_{0} b^{2} \frac{\partial^{2} \bar{N}^{(2)}}{\partial x^{2}} & \approx b^{2} \frac{\partial^{2}}{\partial x^{2}}<R^{(1)}(x, t) N^{(1)}>+b \frac{\partial}{\partial x}<Q^{(1)}(x, t) N^{(1)}> \\
& =b^{2} \frac{\partial^{2}}{\partial x^{2}}\left(-\frac{r_{0} \gamma}{2 k_{B} T_{0}} U e_{0} \sin ^{2}(k x)\right)+b \frac{\partial}{\partial x}\left(\frac{r_{0} b \delta}{4 k_{B} T_{0}} U e_{0} k \sin (2 k x)\right) \\
& =\frac{-r_{0} \gamma b^{2}}{2 k_{B} T_{0}} U e_{0} 2 k^{2} \cos (2 k x)+\frac{r_{0} k^{2} b^{2} \delta}{2 k_{B} T_{0}} U e_{0} \cos (2 k x) \\
& =\frac{r_{0} k^{2} b^{2}(\delta-2 \gamma)}{2 k_{B} T_{0}} U e_{0} \cos (2 k x)
\end{aligned}
$$

The solution to Eq. (16) can be easily found:

$$
\begin{aligned}
\bar{N}^{(2)} & =[1-\exp (-A t)] B \cos (2 k x) \\
& =\left[1-\exp \left(-4 \mathrm{r}_{0} k^{2} b^{2} t\right)\right] \frac{(\gamma-\delta)(\delta-2 \gamma)}{8\left(k_{B} T_{0}\right)^{2}} \frac{\left(k^{2} b^{2} r_{0}\right)^{2}}{\left(k^{2} b^{2} r_{0}\right)^{2}+\Omega^{2}} e_{0}^{2} \cos (2 k x)
\end{aligned}
$$

However, if we recall that for surface Rayleigh waves, the wavenumber and frequency are related by the dispersion relationship $\Omega=k c_{R}$ (where $c_{R}$ is the velocity of the Rayleigh wave), it can for easily be estimated that under "normal" conditions (for materials like silicon or metals like silver) the frequency, to ensure the condition $b^{2} k^{2} r_{0}>\Omega$ it is necessary to use extremely high $\Omega$ for which the wavelength $\lambda=2 \pi / k$ becomes comparable with the atomiccell size. Otherwise, the factor $\left(k^{2} b^{2} r_{0}\right)^{2} /\left[\Omega^{2}+\left(b^{2} k^{2} p_{0}\right)^{2}\right]<<1$ causes extremely strong relaxation damping. In the other words, the contribution from "cascade mechanism" is negligible for the realistic surface acoustic waves.

\subsection{Summary}

Under the standing surface acoustic waves, the adatom diffuses in a way defined by the diffusion barrier perturbed by the acoustic strain field, such that the self-structuring can occur. The self-structuring is described by the Eq. (13), i.e.

$$
\begin{aligned}
\bar{N}(x, t) & \approx\left[1-\exp \left(-4 k^{2} b^{2} r_{0} t\right)\right] \frac{\gamma(\gamma-\delta)}{8\left(k_{B} T_{0}\right)^{2}} e_{0}^{2} \cos (2 k x) & \\
& =[1-\exp (-t / \tau)] \frac{\gamma(\gamma-\delta)}{8\left(k_{B} T_{0}\right)^{2}} e_{0}^{2} \cos (2 k x) & \text { with } \tau=\left(4 k^{2} b^{2} r_{0}\right)^{-1}
\end{aligned}
$$


We only consider the effect of diffusion barrier modification induced by standing surface acoustic waves in the above derivation. The effect of adiabatic temperature induced by standing surface acoustic waves can be easily added by substituting $\gamma$ with $\gamma_{\text {eff }}=\gamma+\gamma_{T}$ in the above equation (as discussed in section 8 and 9):

$$
\begin{aligned}
\bar{N}(x, t) & \approx\left[1-\exp \left(-4 k^{2} b^{2} r_{0} t\right)\right] \frac{\gamma_{e f f}\left(\gamma_{e f f}-\delta\right)}{8\left(k_{B} T_{0}\right)^{2}} e_{0}^{2} \cos (2 k x) \\
& =[1-\exp (-t / \tau)] \frac{\gamma_{e f f}\left(\gamma_{e f f}-\delta\right)}{8\left(k_{B} T_{0}\right)^{2}} e_{0}^{2} \cos (2 k x) \quad \text { with } \tau=\left(4 k^{2} b^{2} r_{0}\right)^{-1}
\end{aligned}
$$




\section{Reference:}

[1] T. H. Maiman, Stimulated optical radiation in ruby, Nature 187, 493-494 (1960)

[2] E. P. Ippen, C. V. Shank, and A. Dienes, Passive mode locking of the cw dye laser, Appl. Phys. Lett. 21(8), 348-350 (1972)

[3] R. L. Fork, C. H. Brito Cruz, P. C. Becker, and C. V. Shank, Compression of optical pulses to six femtoseconds by using cubic phase compensation, Opt. Lett. 12(6), 483-485 (1987)

[4] J. Hecht, Short history of laser development, Appl. Opt. 49, F99-F122 (2010)

[5] D. Bäuerle, Laser Processing and Chemistry, Springer-Verlag, Berlin Heidelberg (2000)

[6] P. A. Molian, Surface alloying using lasers, in T. S. Sudarshan (Ed.), Surface Modification Technologies, Marcel Dekker, New York, , pp. 421-492 (1989)

[7] C. J. Lin, F. Spaepen, Nickel-niobium alloys obtained by picosecond pulsed laser quenching, Acta Metall. 34, 1367-1375 (1989)

[8] J. G. Hoekstra, S. B. Quadri, J. R. Scully, J. M. Fitz-Gerald, Laser surface modification of a crystalline Al-Co-Ce alloy for enhanced corrosion resistance, Adv. Eng. Mat. 7, 805-809 (1989)

[9] K. Biswas, K. Chattopadhyay, Microstructural evolution during laser resolidification of Fe25 atom percent Ge alloy, Metall. Mater. Trans. A 38, 1395-1406 (2007)

[10] S. Yang, Z. J. Wang, H. Kokawa, Y. S. Sato, Grain boundary engineering of 304 austenitic stainless steel by laser surface melting and annealing, J. Mater. Sci. 42, 847-853 (2007)

[11] H. K. Park, C. P. Grigoropoulos, W. P. Leung, and A. C. Tam, A practical excimer laserbased cleaning tool for removal of surface contaminants, IEEE Trans. Compon., Packag. Manuf. Technol., Part A 17, 631-643 (1994)

[12] A. C. Tam, W. P. Leung, W. Zapka, and W. Ziemlich, Laser cleaning technology for removal of surface particulates, J. Appl. Phys. 71, 3515-3523 (1992)

[13] Y.-F. Lu, W.-D. Song, and T.-S. Low, Laser cleaning of micro-particles from a solid surface - theory and applications, Mater. Chem. Phys. 54, 181-185 (1998)

[14] B. S. Lukianchuk (Editor), Laser Cleaning, World Scientific, New Jersey (2002) 
[15] N. G. Basov, E. M. Belenov, M. A. Gubin, M. S. Kurdoglyan, V. V. Nikitin, A. N. Oraevskii, and B. N. Chichkov, New ways of obtaining cold atoms and molecules, Sov. J. Quantum Electron 17, 919-922 (1987)

[16] V. V. Golovelv, S. L. Allman, W. R. Garrett, and C. H. Chen, Laser-induced acoustic desorptiong of electrons and ions, Appl. Phys. Lett. 71, 852-854 (1997)

[17] V. V. Golovlev, S. L. Allman, W. R. Garrett, N. I. Taranenko, and C. H. Chen, Laserinduced acoustic desorption, Int. J. Mass Spectrom. 169, 69-78 (1997)

[18] A. V. Zinovev, I. V. Veryovkin, J. F. Moore, M. J. Pellin, Laser-driven acoustic desorption of organic molecules from back-irradiated solid foils, Anal. Chem. 79, 8232-8241 (2007)

[19] J.-M. Savolainen, M. S. Christensen, and P. Balling, Material swelling as the first step in the ablation of metals by ultrashort laser pulses, Phys. Rev. B 84, 193410 (2011)

[20] M. Christensen, Femtosecond laser ablation of single-crystalline silver under ultra-high vacuum conditions, master thesis, pp. 41-53, Aarhus University, Denmark (2011)

[21] R. M. White, Generation of elastic waves by transient surface heating, J. Appl. Phys. 34, 3559-3567 (1963)

[22] D. A. Hutchins and D. E. Wikins, Elastic waveforms using laser generation and electronmagnetic acoustic transducer detection, J. Appl. Phys. 58, 2469-2477 (1985)

[23] D. Scheider and D. Tucker, Non-destructive characterization and evaluation of thin films by laser-induced ulstrasonic surface waves, Thin Solid Films 290/291, 305-311 (1996)

[24] C. Thomsen, H. Grahn, H. J. Maris, and J. Tauc, Surface generation and detection of phonons by picosecond light pulses, Phys. Rev. B 34, 4129-4138 (1986)

[25] C. Rossignol, J. M. Rampnoux, M. Perton, B. Audoin, and S. Dilhaire, Generation and detection of shear acoustic waves in metal sub-micrometric films with ultrashort laser pulses, Phys. Rev. Lett. 94, 166106 (2005)

[26] Q. Feng, Y. N. Picard, H. Liu, S. M. Yalisove, G. Mourou, T. M. Pollock, Femtosecond laser micromachining of a single-crystal superalloy, Scr Mater. 53, 511-516 (2005)

[27] T. Höche, D. Ruthe, T. Petsch, Low-fluence femtosecond-laser interaction with a Mo/Si multilayer stack, Appl. Phys. A 79, 961-963 (2004) 
[28] J. Jia, M. Li, C. V. Thompson, Amorphization of silicon by femtosecond laser pulses, Appl. Phys. Lett. 84, 3205-3207 (2004)

[29] V. Margetic, K. Niemax, R. Hergenröder, Application of femtosecond laser ablation timeof-flight mass spectrometry to in-depth multilayer analysis, Anal. Chem. 75, 3435-3439 (2003)

[30] R. L. Harzic, N. Huot, E. Audouard, C. Jonin, P. Laporte, Comparison of heat-affected zones due to nanosecond and femtosecond laser pulses using transmission electronic microscopy, Appl. Phys. Lett. 80, 3886-3888 (2002)

[31] C. Xie, Evaluation of alloy element redistribution within laser-melted layer, Surf. Coat. Technol. 113, 1-4 (1999)

[32] T. Sameshima, S. Usui, Analysis of dopant diffusion in molten silicon induced by a pulsed Excimer laser, Jpn. J. Appl. Phys. 26, L1208-L1210 (1987)

[33] E. Ohmura, I. Fukumoto, I. Miyamoto, Molecular dynamics simulation of laser ablation of metal and silicon, Int. J. Jpn. Soc. Precis. Eng. 32, 248-253 (1998)

[34] D. S. Ivanov, L. V. Zhigilei, Combined atomistic-continuum modeling of short pulse laser melting and disintegration of metal films, Phys. Rev. B 68, 064114 (2003)

[35] X. W. Wang, Large-scale molecular dynamics simulation of surface nanostructuring with a laser-assisted scanning tunneling microscope, J. Phys. D: Appl. Phys. 38, 1805-1823 (2005)

[36] A. K. Upadhyay, H. M. Urbassek, Melting and fragmentation of ultra-thin metal films due to ultrafast laser irradiation: a molecular-dynamics study, J. Phys. D: Appl. Phys. 38, 2933$2941(2005)$

[37] C. Cheng, X. Xu, Mechanisms of decomposition of metal during femtosecond laser ablation, Phys. Rev. B 72, 165415 (2005)

[38] S. I. Anisimov, V. V. Zhakhovskii, N. A. Inogamov, K. Nishihara, Yu. V. Petrov, Simulation of the expansion of a crystal heated by an ultrashort laser pulse, Appl. Surf. Sci. 253, 6390-6393 (2007)

[39] B. J. Demaske, V. V. Zhakhovsky, N. A. Inogamov, I. I. Oleynik, Ablation and spallation of gold films irradiated by ultrashort laser pulses, Phys. Rev. B 82, 064113 (2010) 
[40] Z. Lin, R. A. Johnson, L. V. Zhigilei, Computational study of the generation of crystal defects in a BCC metal target irradiated by short laser pulses, Phys. Rev. B 77, 214108 (2008)

[41] D. S. Ivanov, Z. Lin, B. Rethfeld, G. M. O'Connor, Th. J. Glynn, L. V. Zhigilei, Nanocrystalline structure of nanobump generated by localized photo-excitation of metal film, J. Appl. Phys. 107, 013519 (2010)

[42] Z. Lin, E. M. Bringa, E. Leveugle, L. V. Zhigilei, Molecular dynamics simulation of laser melting of nanocrystalline Au, J. Phys. Chem. C 114, 5686-5699 (2010)

[43] L. V. Zhigilei, Z. Lin, D. S. Ivanov, Atomistic modeling of short pulse laser ablation of metals: Connections between melting, spallation, and phase explosion, J. Phys. Chem. C 113, 11892-11906 (2009)

[44] D. A. Thomas, Z. Lin, L. V. Zhigilei, E. L. Gurevich, S. Kittel, R. Hergenröder, Atomistic modeling of femtosecond laser-induced melting and atomic mixing in $\mathrm{Au}$ film - $\mathrm{Cu}$ substrate system, Appl. Surf. Sci. 255, 9605-9612 (2009)

[45] Z. Lin, L. V. Zhigilei, V. Celli, Electron-phonon coupling and electron heat capacity of metals under conditions of strong electron-phonon nonequilibrium, Phys. Rev. B 77, 075133 (2008)

[46] J. Hohlfeld, S.-S.Wellershoff, J. Gudde, U. Conrad, V. Jahnke, and E. Matthias, Electron and lattice dynamics following optical excitation of metals, Chem. Phys. 251, 237-258 (2000)

[47] M. Bonn, D. N. Denzler, S. Funk, M. Wolf, S. -S. Wellershoff, and J. Hohlfeld. Ultrafast electron dynamics at metal surfaces: Competition between electron-phonon coupling and hot-electron transport, Phys. Rev. B 61,1101-1105 (2000)

[48] N. W. Ashcroft, N. D. Mermin, Solid State Physics (Holt, Rinehart and Winston, New York, 1976)

[49] S. I. Anisimov, B. L. Kapeliovich, and T. L. Perel'man, Electron emission from metal surfaces exposed to ultrashort laser pulses, Sov. Phys. JETP 39, 375-377 (1974) 
[50] B. Rethfeld, A. Kaiser, M. Vicanek, and G. Simon, Ultrafast dynamics of nonequilibrium electrons in metals under femtosecond laser irradiation, Phys. Rev. B 65, 214303 (2002)

[51] L. V. Zhigilei and B. J. Garrison, Pressure waves in microscopic simulations of laser ablation, Mater. Res. Soc. Symp. Proc. 538, 491-496 (1999)

[52] C. Schafer, H, H. M. Urbassek, L. V. Zhigilei, and B. J. Garrison, Pressure-transmitting boundary conditions for molecular dynamics simulations, Comp. Mater. Sci. 24, 421-429 (2002)

[53] L. V. Zhigilei and D. S. Ivanov, Channels of energy redistribution in short-pulse laser interactions with metal targets, Appl. Surf. Sci. 248, 433-439 (2005)

[54] http://www.faculty.virginia.edu/CompMat/electron-phonon-coupling/

[55] C. Wu, D. A. Thomas, Z. Lin, and L. V. Zhigilei, Runaway lattice-mismatched interface in an atomistic simulation of femtosecond laser irradiation of $\mathrm{Ag}$ film - $\mathrm{Cu}$ substrate system, Appl. Phys. A 104, 781-792 (2011)

[56] R. H. M. Groeneveld, R. Sprik, A. Lagendijk, Femtosecond spectroscopy of electronelectron and electron-phonon energy relaxation in Ag and Au, Phys. Rev. B 51, 1143311445 (1995)

[57] K. C. Mills, B. J. Monaghan, B. J. Keene, Thermal conductivities of molten metals: Part 1 Pure metals, Int. Mater. Rev. 41, 209-242 (1996)

[58] M. S. Daw, and M. I. Baskes, Embedded-atom method: Derivation and application to impurities, surfaces and other defects in metals, Phys. Rev. B 29, 6443 (1984)

[59] S. M. Foiles, M. I. Baskes, M. S. Daw, Embedded-atom-method functions for the FCC metals Cu, Ag, Au, Ni, Pd, Pt and their alloys, Phys. Rev. B 33, 7983 (1986)

[60] S. M. Foiles, J. B. Adams, Thermodynamic properties of FCC transition metals as calculated with the embedded atom method, Phys. Rev. B 40, 5909 (1989)

[61] A. F. Voter, S. P. Chen, Accurate interatomic potentials for Ni, Al, and Ni3Al, Mater. Res. Soc. Symp. Proc. 82, 175-180 (1999)

[62] J. R. Morris, C. Z. Wang, K. M. Ho, and C. T. Chan, Melting line of aluminium from simulations of coexisting phases, Phy. Rev. B 49, 3109 (1994) 
[63] J. R. Morris, and X. Song, The melting lines of model systems calculated from coexisting simulations, J. Chem, Phys. 116, 9352 (2002)

[64] E. B. Webb III, G. S. Grest, D. R. Heine, J. J. Hoyt, Dissolutive wetting of Ag on Cu: A molecular dynamics simulation study, Acta Mater. 53, 3163-3177 (2005)

[65] J. J. Hoyt, J. W. Garvin, E. B. Webb III, M. Asta, An embedded atom method interatomic potential for the Cu-Pb system, Model. Simul. Mater. Sci. Eng. 11, 287-299 (2003)

[66] Handbook of Chemistry and Physics, 64th edn., R. C. Weast (editor) (CRC Press, Boca Raton, 1983)

[67] R. Najafabadi, D. J. Srolovitz, E. Ma, M. Atzmon, Thermodynamic properties of metastable Ag-Cu alloys, J. Appl. Phys. 74, 3144-3149 (1993)

[68] M. Asta, S. M. Foiles, Embedded-atom-method effective-pair-interaction study of the structural and thermodynamic properties of $\mathrm{Cu}-\mathrm{Ni}, \mathrm{Cu}-\mathrm{Ag}$, and $\mathrm{Au}-\mathrm{Ni}$ solid solutions, Phys. Rev. B 53, 2389-2404 (1996)

[69] J. P. Rogers III, P. Wynblatt, S. M. Foiles, M. I. Baskes, Monte Carlo simulation of the CuAg (001) semicoherent interphase boundary, Acta Metall. Mater. 38, 177-184 (1990)

[70] H. Li, D. Tian, J. Quinn, Y. S. Li, F. Jona, P. M. Marcus, Low-energy electron diffraction and photoemission study of epitaxial films of $\mathrm{Cu}$ on $\mathrm{Ag}\{001\}$, Phys. Rev. B 43, 6342-6346 (1991)

[71] M. Dietterle, T. Will, D. M. Kolb, The initial stages of Cu electrodeposition on $\operatorname{Ag}(100)$ : An in situ STM study, Surf. Sci. 396, 189-197 (1998)

[72] M. Von Allmen, A. Blatter, Laser-Beam interactions with materials: Physical Principles and Applications, Springer, Berlin, (1995)

[73] P. Duwez, R. H. Willens, W. Klement, Continuous series of metastable solid solutions in silver-copper alloys, J. Appl. Phys. 31, 1136-1137 (1960)

[74] R. K. Linde, Lattice parameters of metastable silver-copper alloys, J. Appl. Phys. 37, 934 (1966)

[75] S. Mader, A. S. Nowick, H. Widmer, Metastable evaporated thin films of $\mathrm{CuAg}$ and CoAu alloys-I occurrence and morphology of phases, Acta Metall. 15, 203-214 (1967) 
[76] B. Cantor, R. W. Cahn, Vapour-quenched Ag-Cu alloys, Scr Metall. 10, 318-382 (1976)

[77] H. W. Sheng, G. Wilde, E. Ma, The competing crystalline and amorphous solid solutions in the Ag-Cu system, Acta Mater. 50, 475-488 (2002)

[78] M. Von Allmen, E. Huber, A. Blatter, K. Affolter, Melt quenching at $10^{10} \mathrm{~K} / \mathrm{s}$, Int. J. Rapid Solidif. 1, 15-25 (1984/85)

[79] To avoid misidentification of crystalline structures due to small lattice distortions, the following changes have been made to the method suggested in Ref. [80]: the neighbor radii used for finding neighboring atoms are extended from $r_{i j}{ }^{2}<1.45 r_{0}{ }^{2}$ to $r_{i j}{ }^{2}<1.69 r_{0}{ }^{2}$ for $N_{O}$ neighbors, and from $r_{i j}{ }^{2}<1.55 r_{0}^{2}$ to $r_{i j}{ }^{2}<1.76 r_{0}{ }^{2}$ for $N_{l}$ neighbors; the maximum $\cos \theta_{\text {jik }}$ for $\chi_{3}$ in Table I is changed from -0.705 to -0.195 ; in step (v) of Table II, $\chi_{0}>0$ is changed to $\chi_{7}>0$; in step (vi) of table II, the condition for FCC atoms $\chi_{0}=6$ is made stronger by adding an additional condition $\chi_{0}+\chi_{1}+\chi_{2}+\chi_{3}=30$, and the condition for BCC atoms $\chi_{0}=7$ is made stronger by adding an additional condition $\chi_{0}+\chi_{1}+\chi_{2}+\chi_{3}>30$; the expression $\delta_{\mathrm{HCP}}=\left(\left|\chi_{0}-3\right|+\left|\chi_{0}+\chi_{1}+\chi_{2}+\chi_{3}-9\right|\right) / 12$ is changed to $\delta_{\mathrm{HCP}}=\left(\left|\chi_{0}-3\right|+\left|\chi_{0}+\chi_{1}+\chi_{2}-9\right|\right) / 12$.

[80] G. J. Ackland, A. P. Jones, Applications of local crystal structure measures in experiment and simulation, Phys. Rev. B 73, 054104 (2006)

[81] C. Suárez, W. E. Bron, T. Juhasz, Dynamics and transport of electronic carriers in thin gold films, Phys. Rev. Lett. 75, 4536-4539 (1995)

[82] E. Leveugle, D. S. Ivanov, L. V. Zhigilei, Photomechanical spallation of molecular and metal targets: molecular dynamics study, Appl. Phys. A 79, 1643-1655 (2004)

[83] T. Q. Qiu, C. L. Tien, Femtosecond laser heating of multilayer metals - I. Analysis, Int. J. Heat Mass Transfer 37, 2789-2797 (1994)

[84] E. C. Bain, The nature of martensite, Trans. Am. Inst. Min. Metall. Eng. 70, 25-46 (1924)

[85] Z. S. Pereira, E. Z. da Silva, Martensitic transformation of $\mathrm{Cu}$ on $\mathrm{Ag}(001)$ and $\mathrm{Cu}$ on Au(001) studied with classical molecular dynamics, Phys. Rev. B 79, 115404 (2009)

[86] J. R. Chelikowsky, M. Y. Chou, Electronic and structural properties of elemental copper: A pseudopotential-local-orbital calculation, Phys. Rev. B 38, 7966-7971 (1988) 
[87] Z. Tang, M. Hasegawa, Y. Nagai, M. Saito, Density functional study on metastable BCC copper: Electronic structure and momentum density of positron-electron pairs, Phys. Rev. B 65, 195108 (2002)

[88] A. Kelly, G. W. Groves, P. Kidd, Crystallography and Crystal Defects, revised edn ( Wiley, Chichester, 2000), pp. 257-265.

[89] R. G. Hoagland, T. E. Mitchell, J. P. Hirth, H. Kung, On the strengthening effects of interfaces in multilayer FCC metallic composites, Philos. Mag. A 82, 643-664 (2001)

[90] P. Bacher, P. Wynblatt, S. M. Foiles, A Monte Carlo study of the structure and composition of (001) semicoherent interphase boundaries in Cu-Ag-Au alloys, Acta Metall. Mater. 39, 2681-2691 (1991)

[91] P. Bacher, G. Rao, P. Wynblatt, Comparison of the behavior of (111) and (001) interphase boundaries in $\mathrm{Cu}-\mathrm{Ag}$ and $\mathrm{Cu}-\mathrm{Ag}-\mathrm{Au}$ alloys, Comput. Mater. Sci. 1, $42-50$ (1992)

[92] L. A. Bruce and H. Jaeger, Geometric factors in FCC and BCC metal-on-metal epitaxy. Part 1. Deposits of $\mathrm{Cu}$ and Ni on (001) Ag, Philos. Mag. 36, 1331 (1977)

[93] G. C. Smith, C. Norris and C. Binns, Angle-resolved photoemission from a copper monolayer on silver (100), J. Phys. C 17, 4389 (1984)

[94] Z. Q. Wang, S. H. Lu, Y. S. Li, F. Jona and P. M. Marcus, Epitaxial growth of a metastable modification of copper with body-centered-cubic structure, Phys. Rev. B 35, 9322 (1987)

[95] J. B-Nielsen, J-M. Savolainen, M. Christensen, and P. Balling, Ultra-short pulse laser ablation of copper, silver and tungsten: experimental data and two-temperature model simulations, Appl. Phys. A 103, 447-453 (2011)

[96] Paltauf, G.; Dyer, P. E., Photomechanical processes and effects in ablation, Chem. Rev. 103, 487 (2003)

[97] A. Miotello, and R. Kelly, Critical assessment of thermal models for laser sputtering at high fluences, Appl. Phys. Lett. 67, 3535-3537 (1995)

[98] A. Miotello, and R.Kelly, Laser-induced phase explosion: new physical problems when a condensed phase approaches the thermodynamic critical temperature, Appl. Phys. A 69, S67-S73.(1999) 
[99] N. M. Bulgakova and A. V. Bulgakov, Pulsed laser ablation of solids: transition from normal vaporization to phase explosion, Appl. Phys. A 73, 199-208 (2001)

[100]K. H. Song, and X. Xu, Explosive phase transformation in excimer laser ablation, Appl. Surf. Sci. 127-129, 111-116 (1998)

[101]C.Porneala and D. A. Willis, Observation of nanosecond laser-induced phase explosion in aluminium, Appl. Phys. Lett. 89, 211121 (2006)

[102]B. J. Garrison, T. E. Itina, and L.V. Zhigilei, Limit of overheating and the threshold behavior in laser ablation, Phys. Rev. E 68, 041501 (2003)

[103]R. W. Ohse, and H. V. Tippelskirch, The critical constants of the elements and of some refractory materials with high critical temperatures (A review), High temp. - High Press. 9 , 367-385 (1997)

[104] Y. Gao, L. Song, P. Jiang, L. F. Liu, X. Q. Yan, Z. P. Zhou, D. F. Liu, J. X. Wang, H. J. Yuan, Z. X, Zhang, X. W. Zhao, X. Y. Zhou, W. Y. Zhou, G. Wang, S. S. Xie, H. Y. Chen, and J. Q. Li, Silver nanowires with five-fold symmetric cross-section, J. Crystal Growth 276, 606-612 (2005)

[105]Y. Gao, P. Jiang, L. Song, J. X. Wang, L. F. Liu, D. F. Liu, Y. J. Xiang, Z. X. Zhang, X. W. Zhao, X. Y. Dou, S. D. Luo, W. Y. Zhou, S. S. Xie, Studies on silver nanodecahedrons synthesized by RVP-assisted N, N-dimethylformamide (DMF) reduction, J. Crystal Growth 289, 376-380 (2006)

[106] V. G. Gryaznov, J. Heydenreich, A. M. Kaprelov, S. A. Nepijko, A. E. Romanov, J. Urban, Pentagonal symmetry and disclinations in small particals, Cryst. Res. Technol. 34, 10911119 (1999)

[107]A. L. Kolesnikova and A. E. Romanov, Stress relaxation in pentagonal whiskers, Tech. Phys. Lett. 33, 886-888 (2007)

[108]A. E. Romanov, and A. L. Kolesnikova, Application of disclination concept to solid structures, Prog. Mater. Sci. 54, 740-769 (2009) 
[109]L. M. Dorogin, S. Vlassov, A. L. Kolesnikova, I. Kink, R. Lohmus, and A. E. Romanov, Crystal mismatched layers in pentagonal nanorods and nanoparticles, Phys. Status Solidi B 257, 288-298 (2010)

[110]A. E. Romanov, A. A. Vikarchuk, A. L. Kolesnikova, L. M. Dorogin, I. Kink and E. C. Aifantis, Structural transformations in nano- and microobjects triggered by disclinations, $J$. Mater. Res. 27, 545-55 (2012)

[111]A. Y. Vorobyev and C. Guo, Enhanced absorptance of gold following multipulse femtosecond laser ablation, Phys. Rev. B 72, 195422 (2005)

[112]A. Y. Vorobyev and C. Guo, Effects of nanostructure-covered femtosecond laser-induced periodic surface structures on optical absorptance of metals, Appl. Phys. A 86, 321-324 (2007)

[113]Q.-Z. Zhao, S. Malzer, and L.-J. Wang, Self-organized tungsten nanospikes grown on subwavelength ripples induced by femtosecond laser pulses, Opt. Express 15, 15741-15746 (2007)

[114]Y. Dai, M. He, H. Bian, B. Lu, X. Yan, and G. Ma, Femtosecond laser nanostructuring of silver film, Appl Phys. A 106, 567-574 (2012)

[115]F. A. McDonald, Practical quantitative theory of photoacoustic pulse generation, Appl. Phys. Lett. 54, 1504-1506 (1989)

[116]A. M. Aindow, R. J. Dewhurst, D. A. Hutchins, and S. B. Palmer, Laser-generated ultrasonic pulses at free metal surfaces, J. Acoust. Soc. Am. 69, 449 (1981)

[117]A. Harata, H. Nishimura, and T. Sawada, Laser-induced surface acoustic waves and photothermal surface gratings generated by crossing two pulsed laser beams, Appl. Phys. Lett 57, 132-134 (1990)

[118]R. J. Dewhurst, D. A. Hutchins, and S. B. Palmer, Quantitative measurements of lasergenerated acoustic waveforms, J. Appl. Phys. 53 (6), 4064-4071 (1982)

[119]M. Ochiai, Laser-induced surface acoustic wave technique for precise depth measurement of stress corrosion cracking, J. Phys.: Conf. Ser. 278, 01209 (2011) 
[120]X. Q. Ma, Y. Mizutani, and M. Takemoto, Laser-induced surface acoustic waves for evaluation of elastic stiffness of plasma sprayed materials, J. Mater. Sci. 36, 5633-5641 (2001)

[121]K. A. Nelson, and M. D. Fayer, Laser induced phonons: A probe of intermolecular interactions in molecular solids, J. Chem. Phys. 72, 5202-5218 (1980)

[122]J. A. Rogers, L. Dhar, and K. A. Nelson, Noncontact determination of transverse isotropic elastic moduli in polyimide thin films using a laser based ultrasonic method, Appl. Phys. Lett. 65, 312-314 (1994)

[123]R. I. Tobey, M. E. Siemens, M. M. Murnane, H. C. Kapteyn, D. H. Torchinsky, and K. A. Nelson, Tranisent grating measurement of surface acoustic waves in thin metal films with extreme ultraviolet radiation, Appl. Phys. Lett. 89, 091108 (2006)

[124]M. E. Siemens, Q. Li, M. M. Murnane, H. C. Kapteyn, R. Yang, E. H. Anderson, and K. A. Nelson, High-frequency surface acoustic wave propagation in nanostructures characterized by coherent extreme ultraviolet beams, Appl. Phys. Lett. 94, 093103 (2009)

[125]M. Schubert, M. Grossmann, O. Ristow, M. Hettich, A. Bruchhausen, E. C. S. Barretto, E. Scheer, V. Gusev, and T. Dekorsy, Spatial-temporally resolved high-frequency surface acoustic waves on silicon investigated by femtosecond spectroscopy, Appl. Phys. Lett. 101, 013108 (2012)

[126] M. Levy, H. E. Bass, and R. Stern, Modern Acoustical Techniques for the Measurement of Mechanical Properties, Academic: New York (2001)

[127] V. V. Kozhushko, A. M. Lomonosov, P. Hess, Intrinsic Strength of Silicon Crystals in Pure- and Combined-Mode Fracture without Precrack. Phys. Rev. Lett. 98, 195505 (2007)

[128] A. M. Lomonosov, P. V. Grigoriev, and P. Hess, Sizing of Partially Closed SurfaceBreaking Microcracks with Broadband Rayleigh Waves. J. Appl. Phys. 105, 084906 (2009)

[129] C. D. Wood, S. D. Evans, J. E. Cunningham, R. O’Rorke, C. Wälti, and A. G. Davies, Alignment of Particles in Microfluidic Systems Using Standing Surface Acoustic Waves. Appl. Phys. Lett. 92, 044104 (2008) 
[130] M. Hennig, J. Neumann, A. Wixforth, J. O. Radler, and M. F. Schneider, Dynamic Patterns in a Supported Lipid Bilayer Driven by Standing Surface Acoustic Waves. Lab Chip 9, 3050-3053 (2009)

[131] Y. Inoue, Y. Matsukawa, and K. Sato, Effect of Surface Acoustic Wave Generated on Ferroelectric Support upon Catalysis. J. Amer. Chem. Soc. 111, 8965-8966 (1989)

[132] Y. Watanabe, Y. Inoue, and K. Sato, Activation of a Thin Film Pd Catalyst for Co and Ethanol Oxidation by Surface Acoustic Waves. Surf. Sci. 357-358, 769-772 (1996)

[133] S. Kelling, T. Mitrelias, Y. Matsumoto, V. P. Ostanin, and D. A. King, Acoustic Wave Enhancement of the Catalytic Oxidation of Carbon Monoxide over Pt $\{110\}$. J. Chem. Phys. 107, 5609-5612 (1997)

[134] S. Kelling, S. Cerasari, H. H. Rotermund, G. Ertl, and D. A. King, A Photoemission Electron Microscopy (PEEM) Study of the Effect of Surface Acoustic Waves on Catalytic CO Oxidation over Pt $\{110\}$. Chem. Phys. Lett. 293, 325-330 (1998)

[135] N. Saito, H. Nishiyama, and Y. Inoue. Acoustic Wave Effects on Catalysis: Design of Surfaces with Artificially Controllable Functions for Chemical Reactions. Appl. Surf. Sci. 169-170, 259-263 (2001)

[136] Y. Inoue, Effects of Acoustic Waves-Induced Dynamic Lattice Distortion on Catalytic and Adsorptive Properties of Metal, Alloy and Metal Oxide Surfaces. Surf. Sci. Rep. 62, 305336 (2007)

[137]B. Lindner, and U. Seydel, Laser Desorption Mass Spectrometry of Nonvolatiles under Shock Wave Conditions. Anal. Chem. 57, 895-899 (1985)

[138]A. M. Dow, A. R. Wittrig, and H. I. Kenttämaa, Laser-Induced Acoustic Desorption (LIAD) Mass Spectrometry. Eur. J. Mass Spectrom. 18, 77-92 (2012)

[139] Helvajian, H., private communication (2013)

[140]D. Nardi, E. Zagato, G. Ferrini, C. Giannetti, and F. Banfi, Design of a Surface Acoustic Wave Mass Sensor in the $100 \mathrm{GHz}$ Range. Appl. Phys. Lett. 100, 253106 (2012)

[141]C. Roland, and G. H. Gilmer, Epitaxy on Surfaces Vicinal to Si(001). I. Diffusion of Silicon Adatoms over the Terraces. Phys. Rev. B 46, 13428-13436 (1992) 
[142] C. Ratsch, A. P. Seitsonen, and M. Scheffler, Strain Dependence of Surface Diffusion: Ag on $\operatorname{Ag}(111)$ and $\operatorname{Pt}(111)$. Phys. Rev. B 55, 6750-6753 (1997)

[143] M. Schroeder, and D. E. Wolf, Diffusion on Strained Surfaces. Surf. Sci. 375, 129-140 (1997)

[144]E. Penev; P. Kratzer, and M. Scheffler, Effect of Strain on Surface Diffusion in Semiconductor Heteroepitaxy, Phys. Rev. B 64, 085401 (2001)

[145]D. J. Shu, F. Liu, and X. G. Gong, Simple Generic Method for Predicting the Effect of Strain on Surface Diffusion, Phys. Rev. B 64, 245410 (2001)

[146] A. van de Walle, M. Asta, and P. W. Voorhees, First-Principles Calculation of the Effect of Strain on the Diffusion of Ge Adatoms on Si and Ge(001) Surfaces. Phys. Rev. B 67, $041308(2003)$

[147]S. D. Stoddard, and J. Ford, Numerical Experiments on the Stochastic Behavior of a Lennard-Jones Gas System. Phys. Rev. A 8, 1504-1512 (1973)

[148] L. D. Landau, and E. M. Lifshitz, Theory of Elasticity, $3^{\text {rd }}$ edition; Pergamon Press: Oxford (1986)

[149] R. Stoneley, The Propagation of Surface Elastic Waves in a Cubic Crystal. Proc. R. Soc. Lond. A 232, 447-458 (1955)

[150]H. J. C. Berendsen, J. P. M. Postma, W. F. van Gunsteren, A. DiNola, J. R. Haak, Molecular Dynamics With Coupling to an External Bath. J. Chem. Phys. 81, 3684-3690 (1984)

[151]L. V. King, On the acoustic radiation pressure on spheres, Proc. R. Soc. London, Ser. A 147, 212-240 (1935).

[152]K. Yosioka and Y. Kawasima, Acoustic radiation pressure on a compressible sphere, Acustica 5, 167-173 (1955).

[153]L. D. Landau and E. M. Lifshitz, Mechanics (Pergamon Press, Oxford, 1960).

[154]M. Settnes and H. Bruus, Forces acting on a small particle in an acoustical field in a viscous fluid, Phys. Rev. E 85, 016327 (2012). 
[155]J. Shi et al., D. Ahmed, X. Mao, S.-C. S. Lin, A. Lawit, and T. J. Huang, Acoustic tweezers: patterning cells and microparticles using standing surface acoustic waves (SSAW), Lab Chip 9, 2890-2895 (2009).

[156]Z. Wang and J. Zhe, Recent advances in particle and droplet manipulation for lab-on-a-chip devices based on surface acoustic waves, Lab Chip 11, 1280-1285 (2011).

[157]A. V. Gaponov and M. A. Miller, On the potential well for charged particles in a high frequency electromagnetic field, Zh. Eksp. Teor. Fiz. 34, 242-243 (1958) [Sov. Phys. JETP 7, $168(1958)]$.

[158]B. M. Bolotovskii and A. V. Serov, Details of the motion of charged nonrelativistic particles in a variable field, Physics-Uspekhi 137, 515-516 (1994).

[159] L. P. Gor'kov, On the forces acting on a small particle in an acoustical field in an ideal fluid, Sov. Phys. Dokl. 6, 773-775 (1962).

[160] A. A. Doinikov, Acoustic radiation pressure on a compressible sphere in a viscous fluid, $J$. Fluid Mech. 267, 1-22 (1994).

[161]C. Taillan, N. Combe, and J. Morillo, Nanoscale self-organization using standing surface acoustic waves, Phys. Rev. Lett. 106, 076102 (2011).

[162] N. Combe, C. Taillan, and J. Morillo, Unidimensional model of adatom diffusion on a substrate submitted to a standing acoustic wave. I. Derivation of the adatom motion equation, Phys. Rev. B 85, 155420 (2012).

[163]C. Taillan, N. Combe, and J. Morillo, Unidimensional model of adatom diffusion on a substrate submitted to a standing acoustic wave. II. Solutions of the adatom motion equation, Phys. Rev. B 85, 155421 (2012).

[164]W. T. Coffey, Y. P. Kalmykov, and J. T. Waldron, The Langevin Equation with Applications in Physics, Chemistry and Electrical Engineering, 2nd ed. (World Scientific, Singapore, 2003).

[165]L. D. Landau and E. M. Lifshitz, Theory of Elasticity, 3rd ed. (Pergamon Press, Oxford, 1986). 
[166] C. Wu, V. Yu. Zaitsev, and L. V. Zhigilei, Acoustic enhancement of surface diffusion, J. Phys. Chem. C 117, 9252-9258 (2013)

[167] A. E. Romanov, P. M. Petroff, and J. S. Speck, Lateral ordering of quantum dots by periodic subsurface stressors, Appl. Phys. Lett. 74, 2280-2282 (1999).

[168]Q. Xie, A. Madhukar, P. Chen, and N. P. Kobayashi, Vertically self-organized InAs quantum box islands on GaAs(100), Phys. Rev. Lett. 75, 2542-2545 (1995).

[169] J. Tersoff, C. Teichert, and M. G. Lagally, Self-organization in growth of quantum dot superlattices, Phys. Rev. Lett. 76, 1675-1678 (1996).

[170] M. P. Allen and D. J. Tildesley, Computer simulation of liquids, Clarendon press, Oxford, (1987)

[171] W. Mattson, B. M. Rice, Near-neighbor calculations using a modified cell-linked list method, Comp. Phys, Comm. 119, 135-148 (1999)

[172] J. L. Gustafson, Reevaluating Amdahl's Law, Commun. ACM 31, 532-533 (1988) 
